THE UNIVERSITY OF MANITOBA

\title{
MICROSTRUCTURAL CHARACTERIZATION OF INCOLOY 903 WELDMENTS
}

\section{RAGHUNATH NAKKALIL}

A Dissertation Submitted to the faculty of Graduate Studies in Partial Fulfillment of the requirements of the degree of

\section{DOCTOR OF PHILOSOPHY}

\author{
Metallurgical Sciences Laboratory \\ Department of Mechanical Engineering \\ Winnipeg, Manitoba
}

(C) JANUARY, 1993 
The author has granted an irrevocable non-exclusive licence allowing the National Library of Canada to reproduce, loan, distribute or sell copies of his/her thesis by any means and in any form or format, making this thesis available to interested persons.
L'auteur a accordé une licence irrévocable et non exclusive permettant à la Bibliothèque nationale du Canada de reproduire, prêter, distribuer ou vendre des copies de sa thèse de quelque manière et sous quelque forme que ce soit pour mettre des exemplaires de cette thèse à la disposition des personnes intéressées.
The author retains ownership of the copyright in his/her thesis. Neither the thesis nor substantial extracts from it may be printed or otherwise reproduced without his/her permission.
L'auteur conserve la propriété du droit d'auteur qui protège sa thèse. Ni la thèse ni des extraits substantiels de celle-ci ne doivent être imprimés ou autrement reproduits sans son autorisation. 
Name

Dissertation Abstracts Interturmus. ...... anged by broad, generu suxject categories. Please select the one subject which most nearly describes the content of your dissertation. Enter the corresponding four-digit code in the spaces provided.

MATERIALS SCIENCE SUBJECT TERM
0171914 U.M.I

SUBJECT CODE

\section{Subject Categories}

\section{THE HUMANITIES AND SOCIAL SCIENCES}

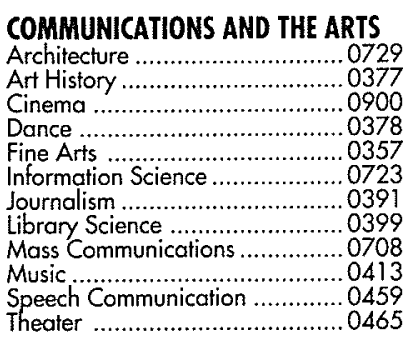

\section{EDUCATION}

General

Administration...........................0515

Adult and Continuing ....................0516

Adult and Continuing $\ldots . \ldots \ldots \ldots \ldots . . . . . .0518$

Agricutural .............................05 0273

Bilingual and Multicultural ............0282

Business …............................0 0688

Community College .................0275

Early Childhood ........................0518

Elementary ................................ 0524

Finance ......................................0277

Guidance and Counseling ........... 0519

Heglth ...................................... 0680

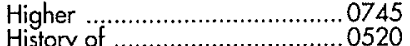

Home Economics .......................

Industrial ................................0521

Language and Literature .............. 0279

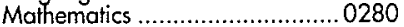

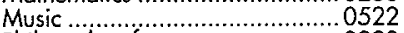

Philosophy of ........................... 0998
Physical ............................ 0523

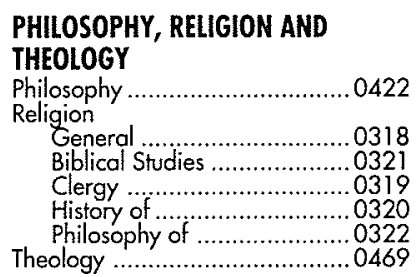

\section{SOCIAL SCIENCES}

American Studies

\section{LANGUAGE, LITERATURE AND} LINGUISTICS

Language

General .............................0679

Ancient ................................... 0289

Linguistics .......................... 0290

Literahure

General .............................040

Classical ................................ 0294

Comparative ....................... 0295

Medieval ...............................0297

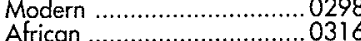

African ............................. 0316

American ....................

Canadian (English) ................0352

Canadian (French) ..............0355

English ..........................0593

Germanic .031

Latin American ....................... 0312

Middle Eastern .................... 0315

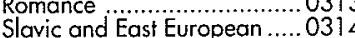

Anthropology

Archaeology ..................... 0324

Cultural ............................0 0326

Business Administration

General ...........................0310

Accounting .......................... 0272

Banking .......................... 0770

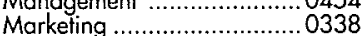

Canadian Studies ......................... 0385

Economics

General ...........................0501

Agricultural ..........................0503

Commerce-Business ................0505

Finance .............................. 0508

History .............................0509

Folklore $\ldots$

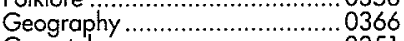

History

General
Labor ............................... 0510

Gerontology .................................. 0351

\section{THE SCIENCES AND ENGINEERING}

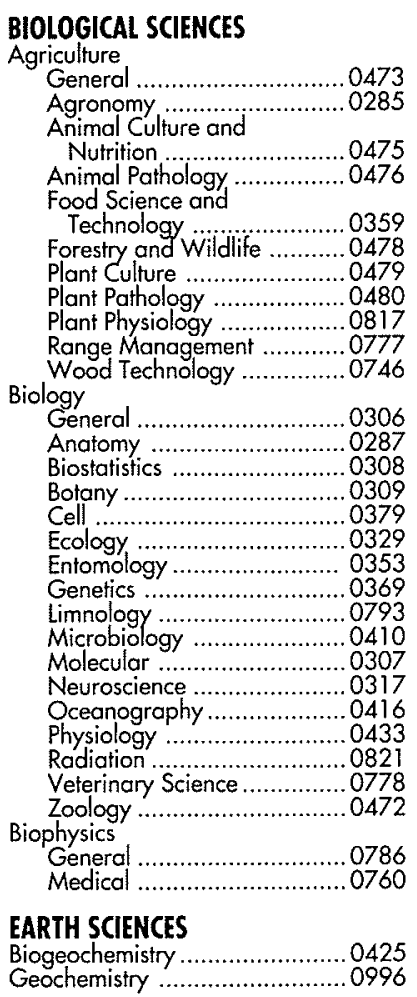

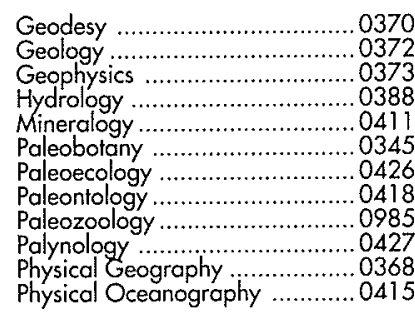

\section{HEALTH AND ENVIRONMENTAL}

\section{SCIENCES}

Environmental Sciences ............0768

Health Sciences

General ............................0566

Audiology ........................... 0300

Chemotherapy .................. 0992

Dentistry .............................0567 0350

Education ......................... 0769

Hospital Management ...........0758

Human Development ...........0758

Medicine and Surgery ..........0564

Mental Health ......................0347

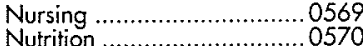

Obstetrics and Gynecology ..0380

Occupational Health and

Therapy .........................0354

Ophtham $\ldots$ logy .....................0381

Ophthalmology ..................057

Pharmacology ........................0419

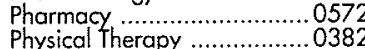

Public Health ........................ 0573

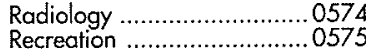

Ancient ..............................0579

Medieval ......................... 058

Modern ............................0582

Black ................................ 0328

Asia, Australia and Oceanio 0332

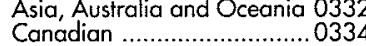

European ............................ 0335

Latin American .................. 0336

Middle Eastern .........

History of Science .......................... 0585

History of Science ..................... 0398

Political Science

General ............................0615

International Law and

Relations .....................0616

Public Administration ............0617

Recreation .............................081

Socia Work ........................... 045

Gociology

General .........................0626

Criminology and Penology ...0627

Demography ................... 0938

Individual and Family

Studies

Industrial and Labor

ndustrial and Labor

Public and Social Welfare.... 0630

Social Structure and

Development ................... 0700

Theory and Methods .............. 0344

Transportation ........................ 0709

Women's Studies .....................0453
Speech Pathology .............0460 Home Economics ..........................0386

PHYSICAL SCIENCES

Pure Sciences

Chemistry

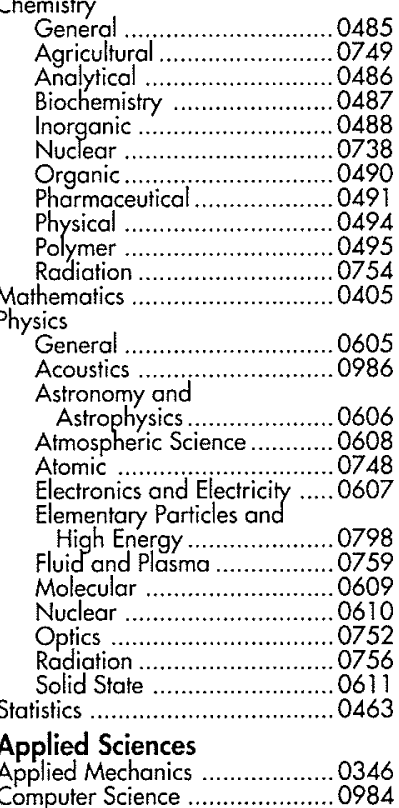

Engineering

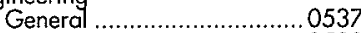

Aerospace ........................0538

Agricultural ........................0539

Automotive ……..................0540

Biomedical .......................... 0541

Chemical ......................... 0542

Electronics and Electrical ....... 0544

Heat and Thermodynamics ...0348

Hydraulic ............................ 0545

Industrial .......................... 0546

Marine $\ldots \ldots \ldots \ldots \ldots \ldots \ldots \ldots . .0547$

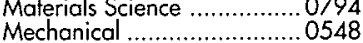

Mechanical .........................0548
Metallurgy ......................... 0743

Mining ……........................ 0551

Nuclear …….................... 0552

Petroleum

Sanitary and Municipal ........0554

System Science.

System Science ....................0490

Operations Research .....................0796

Plastics Technology .....................0795

Textile Technology .......................... 0994

\section{PSYCHOLOGY}

General ................0621

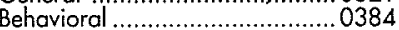

Clinical ……………………......... 0622

Developmental ………................. 0620

Experimental ................................ 0623

Industrial ...................................0624

Personality ................................0625

Physiological .............................0989

Psychobiology ..............................0349

Psychometrics ………………….... 0632

Social ........................................ 0451 


\section{MICROSTRUCTURAL CHARAGTERIZATION OF \\ INCOLOY 903 WELDMENTS}

BY

RAGHUNATH NAKRALIL

A Thesis submitted to the Faculty of Graduate Studies of the University of Manitoba in partial fulfillment of the requirements for the degree of

DOCTOR OF PHILOSOPHY

() 1993

Permission has been granted to the LIBRARY OF THE UNIVERSITY OF MANTTOBA to lend or sell copies of this thesis, to the NATIONAL LIBRARY OF CANADA to microfilm this thesis and to lend or sell copies of the film, and UNTVERSITY MICROFILMS to publish an abstract of this thesis.

The author reserves other publications rights, and neither the thesis nor extensive extracts from it may be printed or otherwise reproduced without the author's permission. 


\section{ACKNOWLEDGEMENTS}

I acknowledge Dr.M.C.Chaturvedi for providing me with the oppurtunity to work on this project. I remain extremely grateful to him not only for accepting me as a doctoral student at a critical juncture of my academic career but also for enduring all my limitations and idiosyncracies ( I am human, all too human...) and providing me with the complete freedom to chart the course of this investigation. I acknowledge Dr.N.L.Richards of Bristol Aerospace Ltd., for his active participation in the project. Many a useful discussions with Dr.J.R.Cahoon are also gratefully acknowledged.

I acknowledge the financial support provided by Manitoba Hydro Co., Winnipeg, in the form of a research fellowship during the course of this investigation. A part of the finances for this research was also provided via a grant from NSERC, Canada and Bristol Aerospace, Winnipeg. My research contributions were made possible by the dedicated assistance of the technical staff with whom I had an oppurtunity to work with, in particular, Mr.P.Mukherji (dada) of IIT, Kanpur, Messrs. Roy Hartle, John Vandorp, Don Mardis and Ron Crampton here. I appreciate and acknowledge their contributions.

Last but not the least, I acknowledge Mr.Horst Weiss for the diligent drafting of all the line drawings and magnification markers, the technical staff at Test and Development Labs, Bristol Aerospace for performing all the electron beam welding and many a friends of mine who have lent me their support during the past nine years of my graduate studies.

Raghu Nakkalil 


\section{ABSTRACT}

The present dissertation reports the findings of an analytical electron microscopy (AEM) characterization of the base metal, the fusion zone and the heat affected zone (HAZ) microstructures in electron beam welded Incoloy 903. The large insolubles in the base metal were observed to be carbides and/or carbonitrides of niobium and titanium. During thermomechanical processing, fine carbides and MNP-type phosphides were observed to precipitate on grain boundaries. The relative proportion of carbides to phosphides increased with an increase in the final solution treatment temperature. During solidification of the fusion zone, $\mathrm{Fe}$ and $\mathrm{Co}$ partitioned into the gamma dendrites, and $\mathrm{Nb}$, $\mathrm{Ti}, \mathrm{P}, \mathrm{Si}$ and $\mathrm{C}$ partitioned into the interdendritic liquid. The solidification was observed to terminate with the gamma/MC carbide eutectic reaction.

Extensive HAZ microfissuring was observed on the warm worked boundaries of the forged and solution treated ring material and on the grain boundaries of the samples solution treated for $1 / 2 \mathrm{~h}$ and $8 \mathrm{~h}$ at $1100^{\circ} \mathrm{C}$. Extensive grain boundary liquid film migration (LFM) was observed on the fine recrystallized grains forming the necklace in the forged and solution treated ring and on the HAZ grain boundaries of the as forged samples solution treated for an hour at $975^{\circ} \mathrm{C}$. The boundaries undergoing LFM were devoid of microfissures. Extensive formation of carbide sheets with arrowhead and lobed morphologies, and as fine precipitates as well as thin solidified films was observed in the microfissures. A solidification pattern similar to that observed in the fusion zone, was observed to occur on the liquated grain boundaries that have subsequently developed microfissures. No resolidified dendritic and interdendritic constituents were observed on boundaries that have undergone LFM.

The observations in the present study suggest that the formation of a continuous liquid film enriched in Nb, $\mathrm{P}$ and $\mathrm{C}$ on the grain boundaries is the major cause for $\mathrm{HAZ}$ 
microfissuring. The liquid originates from the constitutional liquation of the large and fine carbides as well as the Nb-rich MNP-type phosphides. However, if sufficient driving force exists for the liquid film formed on the grain boundaries to relieve its supersaturation by the occurrence of extensive LFM, microfissuring is minimized. HAZ microfissuring occurs in Incoloy 903 due to (a) the formation of a low melting liquid on the boundary (b) the inability of the liquid to relieve the supersaturation by LFM as to the extent required to preclude the occurrence of low melting terminal eutectic liquid prior to crack initiation (c) the adsorption of phosphorus in the liquid to grain interfaces exacerbating the microfissuring by decreasing the solid-liquid interfacial energy and grain boundary cohesion and (d) a straight grain boundary path facilitating easy spreading of the liquid and easy crack propagation. Straight grain boundaries are more susceptible to microfissuring as opposed to curved boundaries due to the decreased driving force for initiation of LFM. 


\section{TABLE OF CONTENTS}

Acknowledgements

i

Abstract $\quad$ ii

Table of Contents $\quad$ iv

List of Figures

List of Tables $\quad$ xvi

Chapter 1: INTRODUCTION 1

Chapter 2: LITERATURE SURVEY 3

2.1: Welding, Weld Defects and Weldability 3

2.1.1: Welding 3

2.1.2: Microstructure of a Weld 3

2.1.3: Weld Defects 8

2.1.4: Weldability 20

2.1.5: Weldability Testing 21

2.1.5.1: Quantitative Measures of Hot Cracking 22

2.1.5.2: The Varestraint Test 23

2.1.5.3: Gleeble Hot Ductility Test 28

2.1.6: Metallurgical Mechanisms of Formation of Weld Defects 35

2.1.6.1: Solidification Cracking in Welds 35

2.1.6.1.1: Review of Theories of Solidification Cracking $\quad 36$

(a) Shrinkage Brittleness theory 37

(b) Strain Theory of Hot Cracking 41

(c) Generalized Theory of Super-Solidus Cracking 51

2.1.6.2: Heat Affected Zone Cracking in Welds 65

2.1.6.2.1: Constitutional Liquation 66

2.1.6.2.2: Grain Boundary Penetration Mechanism 73 
2.1.7: Effect of Base Metal Microstructure on Weldability

2.1.7.1: Solution and Aging Treatment

2.1.7.2: Minor Phase Precipitation on Grain Boundaries

2.1.7.3: Effect of Trace Elements

2.1.7.4: Grain Size

2.2: The 900 Series Superalloys

2.2.1: Introduction

2.2.2: Physical Metallurgy

2.2.3: Weldability of 900 series superalloys 86

2.4: Scope And Nature of the Present Investigation 92

Chapter 3: EXPERIMENTAL PROCEDURES AND TECHNIQUES

3.1: Materials and Processing

3.2: Electron Beam Welding

3.3: X-ray Diffraction

3.4: Optical and Scanning Electron Microscopy

3.5: Analytical Electron Microscopy

Chapter 4: RESULTS AND DISCUSSION

4.1: Base Metal Microstructural Characterization

4.1.1: Introduction

4.1.2: Optical Microscopy

4.1.3: X-ray Diffraction and Scanning Electron Microscopy

4.1.4: Analytical Electron Microscopy

4.2: Characterization of Fusion Zone Microstructure

4.2.1: Introduction

4.2.2: Fusion Zone Microstructure 
4.3: Characterization of $\mathrm{HAZ}$ Microstructures 153

4.3.1: Introduction

4.3.2: HAZ Microstructure-AEM Observations 154

4.3.2.1: Forged and Solution Treated Ring 154

4.3.2.2: Other Microstructural Conditions 164

4.3.3: HAZ Microstructure-Optical and SEM Observations 168

4.4: Grain Boundary Liquid Film Migration 183

4.5: Grain Boundary Embrittlement 208

4.6: Mechanism(s) for HAZ Microfissuring 238

CHAPTER 5: CONCLUSIONS 252

5.1: Base Metal Microstructural Characterization 252

5.2: Characterization of Fusion Zone Microstructure 252

5.3: Characterization of HAZ Microstructures 254

5.4: Mechanism(s) for HAZ Microfissuring 254

$\begin{array}{ll}\text { References } & 259\end{array}$

Referred Journal Publications from the Present Work 268

Author's Vitae $\quad 269$ 


\section{LIST OF FIGURES}

fig 2.1: Microstructure of an Incoloy 903 electron beam weld.- 4.

fig 2.2: A schematic diagram of the various subzones of the HAZ approximately corresponding to the alloy $\mathrm{C}_{0}\left(0.15\right.$ wt \% C) indicated on the $\mathrm{Fe}-\mathrm{Fe}_{3} \mathrm{C}$ diagram [5] -7 .

fig 2.3: Weld discontinuities affecting weld shape and contour (a) undercut and overlapping in a fillet weld (b) undercut and overlapping in a groove weld and (c) underfill in a groove weld [9] 13 .

fig 2.4(a): Simplified sketch of the operation of Varestraint testing device [10] - 24 . fig 2.4(b): Schematic representation of the top surface of the weld showing location of the arc, weld pool and solid-liquid interface at the instant of straining [10] -27.

fig 2.5: Schematic representation of the typical behavior of highly crack resistant materials $[15]-31$.

fig 2.6: Schematic representation of the typical behavior of highly crack sensitive materials [15] -32 .

fig 2.7: Schematic representation of thermally produced strain [15] - 33 .

fig 2.8: Hot cracking susceptibility of eutectiferous alloys [23] - 39.

fig 2.9: Schematic illustration of the process of weld hot cracking [20] - 43.

fig 2.10: Illustrating the nature of strain distribution (extension and contraction) which exists during various stages of solidification in a casting system containing hot spot [20] -46 .

fig 2.11: Illustrating basic concepts of the process by which the total amount and rate of strain (extension imposed on the hot spot zone) concentrated to the liquid films determines the development of hot tearing in castings [20] - 47 .

fig $2.12[29]-49$. 
fig $2.13[29]-50$.

fig 2.14: Effect of constitutional features on cracking susceptibility in binary systems [23] 52.

fig 2.15: Ratio of interphase boundary tension as a function of dihedral angle of the second phase [23] -55 .

fig 2.16: Effect of dihedral angle on (a) shapes of liquid regions (b) distribution of liquid phase on grain corners, edges and faces $[23,32]-56,57$.

fig 2.17: Fracture of solid-liquid components in a weld (thin section). (a) Fracture in liquid $\left(2 \gamma_{L V}\right)$ and fracture at interface $\left(\gamma_{L V}-\gamma_{S V}-\gamma_{S L}\right)$; necking of liquid at free surface due to application of very low strains (c) and (d) liquid film and dendrite formation at the rear end of weld pool during fracture process [25] -62 .

fig 2.18: Schematic diagram of a portion of hypothetical phase diagram for an alloy system exhibiting constitutional liquation [46]. -67 .

fig 2.19(a-c): Schematic representation of the concentration gradients at various temperatures during formation of constitutional liquation [46] - 69 .

fig 2.20(a-c): Schematic representation of the effect of holding a specimen exhibiting constitutional liquation [46] -70 .

fig 2.21: separation between $\gamma^{\prime}$ strengthened alloys that are difficult to weld and those readily weldable [55] -88 .

fig 4.1(a): Optical micrograph of the as received extruded billet of alloy 903 prior to thermo-mechanical processing - 107 .

fig 4.1(b): Optical micrograph of the forged and solution treated ring - 107.

fig $4.1(\mathrm{c})$ : Optical micrograph of the as-forged alloy 903 solution treated at $925^{\circ} \mathrm{C} / 1 \mathrm{~h}$ $-108$.

fig 4.1(d): Optical micrograph of as-forged alloy solution treated at $950^{\circ} \mathrm{C} / 1 \mathrm{~h}-108$. fig 4.2: SEM/BSE image of a large insoluble in the forged and and solution treated ring -114 . 
fig 4.3: SEM/BSE image of showing fine precipitates on the grain boundaries and prior grain boundaries in an as-forged alloy -114 .

fig 4.4(a,b): A TEM, bright field image of fine irregular precipitates on carbon extraction replica from an as-forged alloy -117 .

fig 4.4(c): A TEM, bright field image of a fine irregular precipitate on carbon extraction replica from an as-forged alloy -118 .

fig 4.4(d): SADP from a phosphide, $Z=[10 \overline{1}]-119$.

fig 4.4(e): SADP from a phosphide, $Z=[\overline{1} 11]-119$.

fig 4.4(f): SADP from a phosphide, $Z=[02 \overline{1}]-120$.

fig 4.4(g): A typical STEM/EDS spectrum obtained from particles in fig $4(\mathrm{a}-\mathrm{c})-120$.

fig 4.5. A typical STEM/EDS spectrum obtained from a Niobium rich MX type carbide $-121$.

fig 4.6: A TEM, bright field image of fine carbides on carbon extraction replica from an as-forged alloy solution treated at $975^{\circ} \mathrm{C} / 1 \mathrm{~h}-121$.

fig 4.7(a,b): A TEM bright field image of re-precipitated MNP-type phosphides on carbon extraction replica from a forged alloy solution treated at $975^{\circ} \mathrm{C} / 1 \mathrm{~h}$ and aged for three hours at $850^{\circ} \mathrm{C}-131$.

fig 4.8(a,b): Optical and SEM micrograph of Incoloy 903 aged for $220 \mathrm{~h}$ at $800^{\circ} \mathrm{C}$ 135.

fig 4.9: A typical TEM/EDS spectrum obtained from the unidentified blocky particles in samples aged for long times in the temperature range $750^{\circ} \mathrm{C}-850^{\circ} \mathrm{C}-136$.

fig 4.10: A typical TEM/EDS spectrum obtained from the needle shaped phase in Incoloy 903 aged for $220 \mathrm{~h}$ at $800^{\circ} \mathrm{C}-136$.

fig $4.11(a, b)$ : Optical micrographs illustrating the cellular-dendritic structure observed in the fusion zone - 139 .

fig 4.11 (c): Optical micrograph illustrating the cellular-dendritic structure observed in the fusion zone - 145 . 
fig 4.12(a): Low magnification TEM micrograph of interdendritic carbides from fusion zone extracted on a carbon extraction replica -145 .

fig 4.12(b): TEM micrographs of interdendritic carbides from fusion zone extracted on a carbon extraction replica. The inset in figure $4.12(b)$ shows a [001] zone diffraction pattern obtained from a carbide -146.

fig 4.12(c): Illustrating the characteristic arrow head and lobed morphology of interdendritic carbides -147 .

fig 4.12(d): A typical TEM/EDS spectrum obtained from an interdendritic carbide sheet $-147$.

fig 4.13(a): TEM micrograph of a Laves phase particle observed on the edge of a carbide sheet. The inset shows a [1100] zone diffraction pattern obtained from a Laves phase 148.

fig 4.13(b): A typical TEM/EDS spectrum obtained from a Laves phase particle — 148. fig 4.14: Low magnification TEM micrograph of the fusion zone and HAZ extracted on a carbon replica -156 .

fig 4.15(a-c): TEM micrographs of a HAZ microfissure. Figs 4.15(b,c) show different areas of fig 4.15(a) at a higher magnification - 157.

fig 4.15(d): TEM micrograph showing an interdendritic carbide sheet in a HAZ microfissure -158 .

fig 4.15(e): TEM micrograph showing extensive fine carbide precipitation aside a $H A Z$ microfissure - 158 .

fig 4.16(a): A typical [0001] zone diffraction pattern obtained from an $\mathrm{M}_{4} \mathrm{C}_{2} \mathrm{~S}_{2}$ particle -159 .

fig 4.16(b): A typical TEM/EDS spectrum obtained from an $M_{4} C_{2} S_{2}$ particle - 159 . fig 4.17: TEM micrograph of a grain boundary in HAZ that resisted microfissuring 162.

fig 4.18: TEM micrograph illustrating defect free MNP-phosphide precipitation in the far 
$\mathrm{HAZ}-163$.

fig 4.19(a,b): TEM micrographs (on carbon extraction replica) of the thickened HAZ boundaries observed in case $A$ welds of as-forged samples solution treated at $975^{\circ} \mathrm{C}$ for an hour - 165 .

fig 4.20(a): TEM micrograph showing thin carbide and Laves phase sheets in case A weld $\mathrm{HAZ}$ of forged and solution treated ring further solution treated for $8 \mathrm{~h}$ at $1100^{\circ} \mathrm{C}$ 166.

fig 4.20(b): TEM micrograph of a different area of the same microfissure as in $4.20(\mathrm{a})$ illustrating interdendritic carbides - 166.

fig 4.21(a,b,c): TEM micrographs of a different HAZ grain boundary from the same replica shown in fig 4.20(a). Note the lack of continuous/semicontinuous sheets of terminal solidification constituents on this boundary that resisted microfissuring - 167,171.

fig 4.22(a): Optical micrograph of case A welds (fusion zone and HAZ) of forged and solution treated ring -171 .

fig 4.22(b): Optical micrograph of case A welds (fusion zone and HAZ) of forged and solution treated ring further solution treated for $1 / 2 \mathrm{~h}$ at $1100^{\circ} \mathrm{C}-172$.

fig 4.22(c): Optical micrograph of case A welds (fusion zone and HAZ) of forged and solution treated ring further solution treated for $8 \mathrm{~h}$ at $1100^{\circ} \mathrm{C}-172$.

fig 4.22(d): Optical micrograph of case A welds (fusion zone and HAZ) of as-forged alloy with warm worked boundaries lying nearly parallel to the fusion line -173 .

fig 4.22(e): Optical micrograph of case A welds (fusion zone and HAZ) of as-forged alloy solution treated for an hour at $975^{\circ} \mathrm{C}-173$.

fig 4.23(a): SEM/BSE image of a microfissure in the HAZ - 174.

fig 4.23(b): SEM/BSE image illustrating interdendritic constituents observed in a microfissure -174 .

fig 4.23(c): Niobium x-ray map of a HAZ microfissure - 175 .

Fig 4.24: Optical micrograph showing the thickened appearence of the fine recrystallized 
grains -175 .

Fig 4.25(a-c): Optical micrographs illustrating the pleated morphology of HAZ microfissures - 176 .

fig 4.26: SEM micrographs showing partial primary MC carbide liquation in $H A Z-177$.

Fig 4.27(a): Optical micrograph showing the thickened appearence of the fine recrystallized grains - 177 .

Fig 4.27(b,c): SEM micrographs showing the thickened appearence of the fine recrystallized grains -178 .

Fig 4.27(d,e): SEM micrographs showing the thickened appearence of the recrystallized grains observed in case $A$ welds of as-forged samples solution treated at $975^{\circ} \mathrm{C}$ for an hour -179 .

fig 4.28: Free energy curves for solid and liquid phases in a two component ( $A$ and $B$ ) system: Curve marked $G_{L}$ applies to the liquid phase while the curve marked $G_{S}$ applies to the unstressed solid phase: the usual tangent construction gives the composition of the solid and liquid in contact with each other at equilibrium as $C_{S}$ and $C_{L}$ respectively. For a solid with initial composition $C_{0}$, diffusion of solute into or out of the surface forms an elastically stressed layer, the free energy of which is increased by an amount $G_{E} l$, which varies as the square of the difference in concentration from $C_{0}$. In view of this stressed layer, a new equilibrium compostion at $\bar{C}_{S}$ and $\bar{C}_{L}$ are established -186 .

fig 4.29: Common tangent construction for an unstressed planar solid and a coherently stressed solid in equilibrium with liquid $[130,131]-191$.

fig 4.30: Common tangent construction for an coherent solid with (a) a low and (b) a high elastic energy in equilibrium with liquid $[130,131]-194$.

fig 4.31: Graphical construction of limiting liquid compositions for spontaneous dissolution of solids with $C_{0}, \bar{C}_{S}$ and $C_{S}[130,131]-200$.

fig 4.32: SEM micrograph illustrating some backfilling occurring on some warm worked boundaries lying at an inclination (unlike perpendicular) to the fusion line - 206. 
fig 4.33: Variation in grain boundary energy of a range of binary alloys with bulk solute content [143] -211.

fig 4.34: Grain boundary enrichment factor as a function of the atomic solid solubility for binary systems. The host element is underlined in each case [144] -212 .

fig 4.35(a): The tertahedral dodecahedron [147]- 216 .

fig 4.35(b): The total valance change density for $\mathrm{Ni}_{8}(\mathrm{a})$ and $\mathrm{Ni}_{8} \mathrm{~S}(\mathrm{~b})$. The contours are all equivalued. Note in particular the change in contour number 4 for the two clusters. The locations of the atoms corresponding to the number are given in figure 4.36(a) [147]216.

fig 4.36: Concentration of the four principal tramp elements at the grain boundaries of common commercial steels compared with the bulk content [158] - 219.

fig 4.37: Schematic illustration of grain boundary sweeping in the weld HAZ - 241 .

fig 4.38: SEM micrograph showing HAZ cracking remote from the fusion line -243. 


\section{LIST OF TABLES}

table 2.1: Microstructural conditions investigated

table 3.1(a): Composition of Incoloy 903 (Extruded Billet)

table 3.1(b): Composition of Incoloy 903

Forged and solution treated ring

table 3.2: Fractional factorial experimental parameters

used for electron beam welding

table 4.1: Grain sizes of Incoloy 903

table 4.2: Composition of insolubles and precipitates

122,123

table 4.3: Crystallographic data on precipitates and insolubles

124

table 4.4: Chemical composition (wt \%) of the blocky phase

table 4.5: Chemical composition (at \%) of the delta phase

table 4.6: Chemical composition (wt \%) of the gamma dendrite cores

table 4.7: Partitioning coefficients of various alloying elements

table 4.8: Chemical composition (wt \%) of the Laves phase

table 4.9: Chemical composition (wt \%) of the trace phases

table 4.10: The chemical composition in wt \% of the LFM regions and the matrix as determined by SEM/EDS quantitative microanalyses

table 4.11: List of effects of alloying and tramp elements on the

weldability of nickel-base superalloys compiled by Pease [168]

table 4.12: [59]

table 4.13: Chemical Composition of In 718 and Rene 220C [173] 


\section{Chapter 1}

\section{INTRODUCTION}

Welding is an indispensable fabrication technique for a wide variety of components. However, the emphasis in alloy development is generally to optimize the mechanical properties and the oxidation behavior by suitable alloying additions, processing schedules etc.. The problems associated with joining rarely gain attention during the stages of alloy development and welding engineers are expected to devise procedures and techniques to join each alloy. Welding for a long time, was considered as an art rather than as a science. However, the experience in the past four to five decades has indicated that some of the alloy modifications that emerged for improved mechanical properties led to a deterioration in the weldability of the alloy thus causing exaggerated fabrication cost in some cases.

The problems associated with welding is cracking and deteriorated mechanical properties. Cracking and microfissuring is observed in the weld metal and the heat affected zone during welding and/or post weld heat treatment. A second problem associated with welding is the decrease in mechanical properties and oxidation resistance in the weld metal due to segregation occuring during solidification. Exaggerated grain growth, dissolution and coarsening of precipitates, precipitation of undesired phases, and build up of residual stresses may occur in the heat affected zone leading to a drastic decrease in the mechanical properties. Of the above mentioned problems, the most critical one is the cracking and microfissuring in the HAZ and the weld metal. Though some of the cracking can be eliminated by using correct welding procedures and techniques, the fundamental causes for microfissuring are metallurgical in nature. The present investigation was undertaken with the primary objective of understanding the metallurgical mechanism(s) responsible 
for microfissuring.

The evolution of modern superalloys has coincided with the evolution of turbine engines that power high performance military and commercial aircrafts as well as industrial turbines. High temperature nickel-base and iron-base superalloys are frequently welded into complex assemblies that are required in the fabrication of the jet engine components using both electron beam welding and gas tungsten arc welding. However, high temperature nickel-base superalloys and nickel-iron-base superalloys, in particular the alloys hardened by precipitation, are highly susceptible to solidification cracking, heat affected zone microfissuring and post weld heat treatment cracking. The severe conditions to which these alloys are exposed, makes changes in microstructure and properties as produced by welding of special importance and the avoidance of cracking, imperative.

The 900 series superalloys, based on the $\mathrm{Fe}-\mathrm{Ni}$-Co system, are a relatively new class of aerospace materials that offer high temperature strength and a low coefficient of thermal expansion. These alloys are precipitation hardened. Incoloy 903 is the most widely used of the 900 series alloys, finding extensive use in the manufacture of turbine shroud supports and other parts that require close dimensional tolerances. In recent years, a great deal of microfissuring has been reported in electron beam welded parts made of Incoloy 903.

This investigation was undertaken to understand the physical metallurgy of welding of Incoloy 903. The relationship between base metal microstructure and the fusion zone and heat affected zone microstructures was sought using analytical electron microscopy. From the results of the characterization of various base metal and heat affected zone microstructures, mechanism(s) for heat affected zone microfissuring were aimed to be developed. The effect of precipitation of minor phases and trace elements on the weldability of Incoloy 903 was to be ascertained. The information on the effect of deleterious trace elements on weldability is expected to aid in future alloy developmental projects for improved weldability. 


\section{Chapter 2}

\section{LITERATURE REVIEW}

\section{1: WELDING, WELDABIIITY AND WELD DEFECTS}

\subsection{1: Welding}

Welding is defined as an operation by which two or more parts are united, by means of heat or pressure, or both, in such a way that there is continuity in the nature of the material between these parts. A filler material, the melting temperature of which is of the same order as that of the parent material, may or may not be used.

Fusion Welding: Fusion welding is defined as a welding operation in which the weld is made between materials in a molten state without the application of pressure.

\subsection{2: Microstructure of a Weld}

A typical structure of a weld is shown in fig 2.1. It consists of weld zone (fusion zone), the heat affected zone ( $H A Z)$ and the fusion line demarcating the resolidified fusion zone and the HAZ. Some authors refer to portion of the HAZ immediately adjacent to the fusion zone as the partially melted zone [1]. Fig 2.1 reveals a narrow cast weld zone extending through approximately $90 \%$ of the thickness. This is commonly known as the spike. The remaining portion of the weld has a gradually increasing weld width and is known as the nail head. The area located at the junction of the nail head and the spike is called the shoulder. The shape of the weld metal need not be like an 'hour glass' as shown in fig 2.1. A variety of shapes are observed in welds. However, the 'hour glass' shape shown in fig 2.1 is typical of welds made by electron beam welding with point focus and no beam oscillation. 


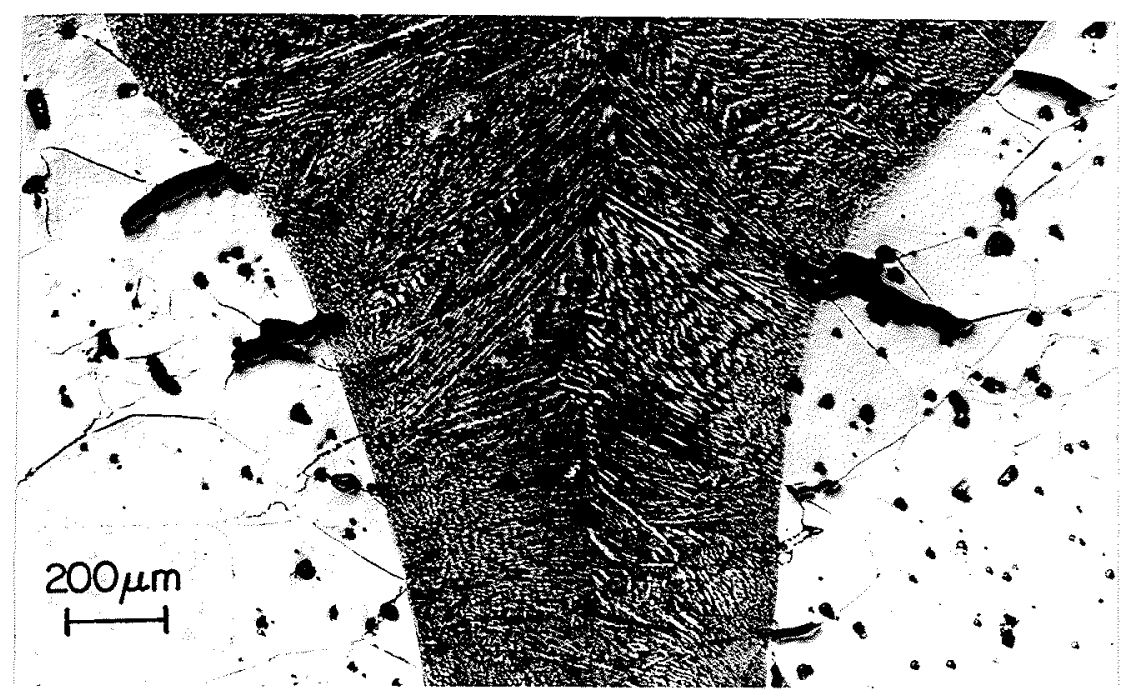

fig 2.1: Microstructure of an Incoloy 903 electron beam weld. 
Fusion Zone: The weld zone is the area where complete melting and resolidification of the grains has occurred. A weld zone may be considered as a small casting, often a continuous casting formed under special conditions: an intensely stirred liquid pool, high temperature gradients, rapid growth rates, intimate contact between the molten alloy and the mold including partial melting of the mold [2]. The solidification in the weld zone does not require nucleation [3]. The molten alloy of the fusion zone freezes within a mold formed of the base metal. The molten alloy, as its heat of fusion is transferred to the base metal, grows epitaxially on the base metal. Each base metal grain on the fusion line serves as a growth site for a fusion zone grain. Thus, the initial grain size of the fusion zone at the fusion line will be the same as the grain size of the base metal. Also, the crystallographic orientation of each fusion zone grain will be the same as the orientation of the base metal grain on which it grows epitaxially.

The grains at the fusion line grow in a direction roughly perpendicular to the fusion line. As the grains grow, there is a competition between the neighbors. Some continue to grow, others terminate. Generally, the surviving grains are the ones that have a preferred crystallographic axis closely aligned with the heat flow direction. In cubic alloys, the directions $<100\rangle$ are the preferred grain directions $[3,4]$. As a result of the competition, the grain size gradually increases with increasing distance from the fusion line.

Any combination of columnar and equiaxed grains may form in a weld zone. Columnar grains are elongated in one direction which locally, is the primary direction of heat flow. Equiaxed grains have about the same width and have random crystallographic orientations. Typically, weld zone grains are either totally columnar or columnar near the fusion line and changing to equiaxed at the center. A transition from columnar to equiaxed grains is observed in large weld zones and in alloys with a wide freezing range. The formation of equiaxed grains, especially fine equiaxed grains, is often desirable in welds and castings.

Heat Affected Zone: When structural members are joined by fusion welding, the material of the plates has to be heated to the melting point and then cooled again rapidly 
under the conditions of restraint imposed by the geometry of the joint. As a result of this very severe thermal cycle, the original microstructure and the properties of the material in a region close to the weld are altered. This volume of the material or zone is usually referred to as the heat affected zone (HAZ).

The HAZ can be conveniently divided into a number of sub-zones (depending on the material being welded) and this is illustrated for the case of a transformable steel in fig 2.2 [5]. Each sub-zone refers to a different type of microstructure and perhaps more important, each microstructural type is likely to possess different mechanical properties. The microstructure type and its sub-zone width are partially dictated by the thermal cycle, i.e., the complete cycle of heating and cooling due to the movement of the arc and the thermal properties of the base material. However, the changes in the HAZ are extremely sensitive to the prior thermal and mechanical history of the base material. For example, recrystallization behavior during the heating cycle is affected by whether the original material was in cold rolled or annealed condition prior to welding. The onset and extent of grain growth are influenced by the presence of precipitates and their solubility at high temperatures. Thus in order to obtain a reasonable understanding of the $\mathrm{HAZ}$ microstructure it is necessary to consider how the microstructure of the base material reacts to the complete thermal cycle, i.e. the heating cycle, the time held at temperature (dwell time or residence time including the effect of the melt zone at the fusion line) and finally, the cooling cycle and its effect on, phase transformations and precipitation reactions.

The final grain size in the grain growth region increases with increasing peak temperature and residence time. In fact the maximum peak temperature is the melting point and it is the grain size at the fusion boundary that is important since it determines the grain size in the weld metal. The only significant variable (assuming same metallurgy) is the residence time. The charts developed by Christensen et. al. [6] show that residence time is roughly proportional to the heat input rate. Thus welding processes with charac- 


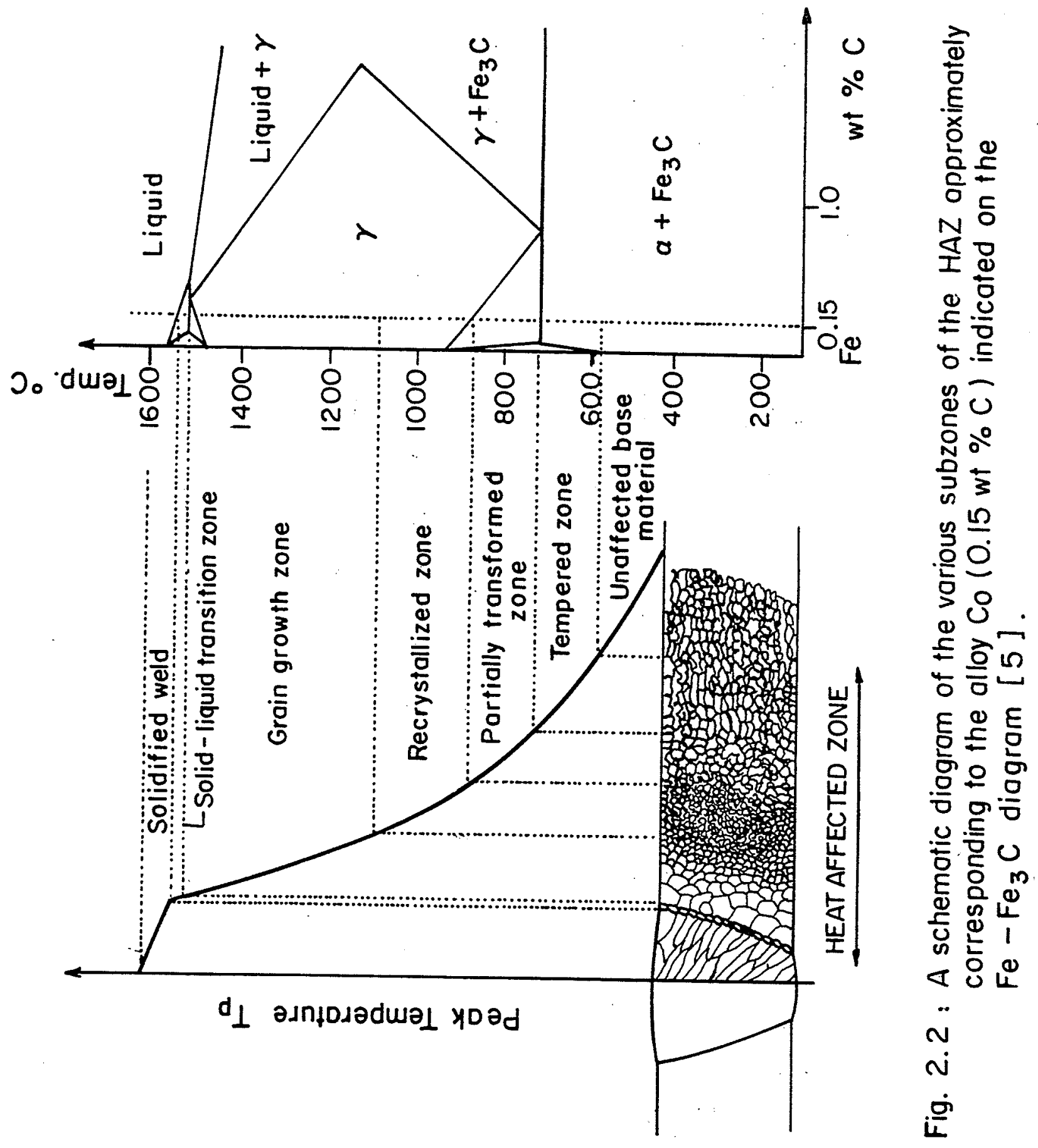


teristically high heat input rates such as electroslag and submerged arc welding generate coarse grain sizes in the HAZ and weld metal. There are two ways in which residence time can be reduced; either by using a welding process that generates a two dimensional heat flow (residence times for two dimensional heat flow are about one half of those for three dimensional heat flow) or by decreasing the heat input rate. Electron beam and laser welding have these characteristics and it is one of the advantage of such processes that they minimize the disturbance in the HAZ [7].

If the metal that is subjected to a weld thermal cycle has been previously hardened by cold work, the grain growth may be preceded by recrystallization which, in turn, results in softening. Copper and aluminum are typical of metals that can acquire a useful increase in mechanical properties by cold work and fusion welding will usually destroy the effect of cold work in the HAZ. Similarly, alloys that are hardened by precipitation will usually be softened in the HAZ by fusion welding.

In addition to the grain growth which may in itself cause embrittlement, there are a number of metallurgical changes that result from the weld thermal cycle that may alter the properties of the HAZ. Hydrogen does not appear to have any significant effect on the properties of austenitic chromium-nickel steel or non-ferrous metals other than copper. Ferritic steel is embrittled to a greater or lesser degree by hydrogen, and copper if not deoxidized, may be severely embrittled by the reaction of hydrogen with residual oxygen to form steam. The steam precipitates at grain boundaries and generates fissures. Ferritic alloy steels may be embrittled by the formation of unfavorable transformation products; by carbide precipitation, by grain boundary segregation (temper brittleness) and by strain aging.

\subsection{3: Weld Defects $[8]$}

"Weld Discontinuities" are interruptions in the desirable physical structure of the weld. A discontinuity constituting a danger to the fitness-for-purpose of a weld is a 'defect'. By 
definition, a defect is a condition that must be removed or corrected. Fitness-for-purpose is a concept of weld evaluation that seeks a balance among quality, reliability and economy of welding procedure. Fitness-for-purpose is not a constant and varies depending on the service requirements of a particular welded structure, as well as on the properties of the material involved.

Imperfections in metallic fusion welds are characterized into six main groups by the welding handbook [9]. These are as follows:

\section{Cracks}

2. Cavities

3. Solid inclusions

4. Lack of fusion and penetration

5. Imperfect shape

6. Miscellaneous imperfections not included in the above groups.

Each of these groups and the defects that form part of, are discussed below following the welding Handbook [7].

\section{Group no 1: Cracks}

a. Cracks: A discontinuity produced by a local rupture which may arise from the effect of cooling or stresses.

b. Microfissure (micro-crack): When a crack has microscopic dimensions.

c. Longitudinal crack: A crack substantially parallel to the axis of the weld. It may be situated (i) in the weld metal (ii) at the weld junction (iii) in the heat affected zone and (iv) in the parent metal.

d. transverse cracks: A crack substantially transverse to the axis of the weld. It may be situated (i) in the weld metal (ii) in the heat affected zone and (iii) in the parent metal. e. Radiating cracks: Cracks radiating from a common point. They may be found (i) in the weld metal (ii) in the heat affected zone and (iii) in the parent metal. Small cracks of this type are known as star cracks. 
f. Crater cracks: A crack in the end crater of a weld which may be (i) longitudinal (ii) transverse and (iii) Star cracking.

g. Group of disconnected cracks: A group of disconnected cracks which may be situated (i) in the weld metal (ii) in the heat affected zone and (iii) in the parent metal.

h. Branching cracks: A group of connected cracks originating from a common crack and distinguishable from disconnected cracks and from radiating cracks. They may be situated (i) in the weld metal (ii) in the heat affected zone and (iii) in the parent metal.

\section{Group no 2: Cavities}

a. Gas cavity: A cavity formed by entrapped gas.

b. Gas pore: A gas cavity of essentially spherical form.

c. Uniformly distributed porosity: A number of gas pores distributed in a substantially uniform manner through out the weld metal. This should not be confused with linear porosity.

d. Localized (clustered) porosity: Group of gas cavities.

e. Linear porosity: A line of gas pores situated parallel to the axis of the weld.

f. Elongated cavity: A large non-spherical cavity with its major dimension approximately parallel to the axis of the weld.

g. Worm-hole: A tubular cavity in the weld metal caused by release of gas. The shape and position of the worm-holes is determined by the mode of solidification and the sources of gas. Generally they are grouped in clusters and distributed in a herringbone formation. h. Surface pore: A small gas pore which breaks the surface of the weld.

i. Shrinkage cavity: A cavity due to shrinkage during solidification.

j. Interdendritic shrinkage: An elongated shrinkage cavity formed between dendrites during cooling which may contain entrapped gas. Such a defect is generally found perpendicular to the weld face.

k. Microshrinkage: Shrinkage only visible under the microscope.

I. Interdendritic microshrinkage: Interdendritic shrinkage only visible under the micro- 
scope.

m. Crater pipe: The depression at the end of a weld run and not eliminated before or during subsequent weld passes.

\section{Group no 3: Solid Inclusions}

a. Solid inclusion: Solid foreign substance entrapped in the weld metal.

b. Slag inclusion: Slag entrapped in the weld metal. According to the circumstances of their formation, such inclusions may be (i) linear (ii) isolated or (iii) others.

c. Flux inclusion: Flux entrapped in the weld metal. According to the circumstances of their formation, such inclusions may be (i) linear (ii) isolated or (iii) others.

d. Oxide inclusion: Metallic oxide trapped in the weld metal during solidification.

e. Puckering: In certain cases, especially in aluminum alloys, gross oxide film enfoldment can occur due to a combination of unsatisfactory protection from atmospheric contamination and turbulance in the weld pool.

f. Metallic inclusion: A particle of foreign metal trapped in the weld metal. It may be of tungsten, copper or any other metal.

\section{Group no 4: Lack of Fusion and Penetration}

a. Lack of fusion (incomplete fusion): Lack of fusion denotes a discontinuity caused by incomplete coalescence of some portion of the filler metal with the base material. It can also form between weld beads in the case of multi-pass welds. These essentially twodimensional flaws occur when insufficient heat is absorbed by the underlying metal from the weld, causing incomplete melting at the interfaces of the weld and the base metal of successive passes. Lack of fusion is usually elongated in the direction of welding, with either sharp or rounded edges, depending on the conditions of formation. Lack of fusion can be one of the following: (i) lack of side wall fusion (ii) lack of inter-run fusion and (iii) lack of fusion at the root of the weld.

b. Lack of penetration (incomplete penetration): When the weld metal has not penetrated to the bottom of the weld joint, the result is lack of penetration. Lack of penetration is 
caused by incorrect welding technique or by improper root gap.

\section{Group no 5: Imperfect Shape}

a. Undercut: Undercut, as shown in fig 2.3 , is a gap located at the toe or the root of a weld that occurs when the weld metal does not completely fill the gap at the surface of the groove to form a smooth junction at the weld toe. Undercut actually refers more to the base metal adjacent to the weld, whereas imperfect shape is a defect of the weld itself. Undercut may be continuous or intermittent. Undercutting is particularly troublesome because it produces stress raisers that create problems under impact, fatigue or low temperature service.

b. Shrinkage groove: A shallow groove in the root caused by the contraction in the weld metal along each side of the penetration bead.

c. Excess weld metal: An excess of weld metal at the face(s) of the butt weld.

d. Excessive convexity: An excess of weld metal at the face of a fillet weld.

e. Excessive penetration: Excess weld metal protruding through the root of a weld made from one side or through weld metal previously deposited from either side of a multi-run joint.

f. Local protrusion: Local excessive penetration.

g. Incorrect weld profile: Too small an angle between the plane of the parent metal surface and a plane tangential to the weld bead surface at the toe.

h. Overlap: Overlapping, as shown in fig 2.3 , is the protrusion of the weld metal over the edge or toe of the weld bead. This defect can cause an area of incomplete fusion which creates a notch and can lead to crack initiation.

i. Linear misalignment: Misalignment between two welded pieces such that whilst their surface planes are parallel they are not at the required level.

j. Angular misalignment: Misalignment between two welded pieces such that their surface planes are not parallel (or at the intended angle).

k. Sagging: Weld metal collapse due to gravity. According to the circumstances it may 

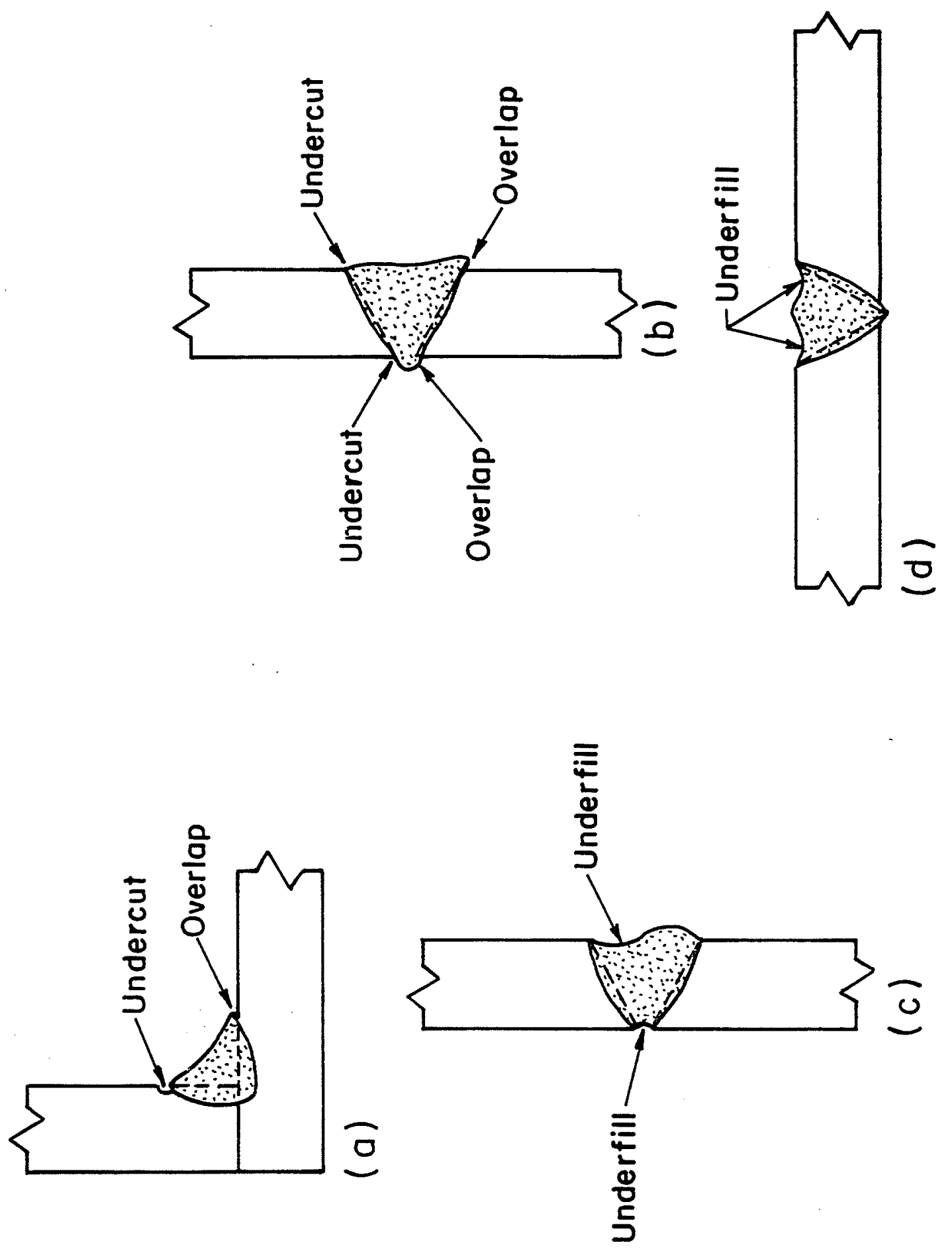

Fig. 2.3 : Weld discontinuities offecting weld shope and cotour [9]. 
be (i) sagging in horizontal-vertical (ii) sagging in flat or overhead (iii) sagging in a fillet weld or (iv) sagging (melting) of the edge.

I. Burn through: A collapse of the weld pool resulting in a hole in the weld or at the side of the weld.

m. Incompletely filled groove: A longitudinal continuous or intermittent channel in the surface of a weld due to insufficient deposition of the weld metal.

n. Excessive assymmetry of fillet weld

o. Irregular width: Excessive variation in width.

p. Irregular surface: excessive surface roughness.

q. Root concavity: A shallow groove due to shrinkage of a butt weld at the root.

r. Root porosity: Spongy formation at the root of a weld due to bubbling of the weld metal at the moment of solidification.

s. Poor restart: A local surface irregularity at a weld restart.

t. Underfill: Underfill is defined as a depression on the face of a weld or the root surface extending below the surface of the adjacent base metal. Underfill, as shown in fig 2.3, reduces the cross sectional area of the weld below the designed value and therefore is a point of weakness and potentially a stress raiser where failure may initiate.

\section{Group no 6: Miscellaneous imperfections}

a. Miscellaneous imperfections: All imperfections that cannot be included in groups 1-5.

b. Stray flash or arc strike: Local damage to the surface of the parent metal adjacent to the weld, resulting from arcing or striking the arc outside the weld groove.

c. Spatter: Globules of weld metal or filler expelled during welding and adhering to the surface of the parent metal or solidified weld metal.

d. Tungsten spatter: Particles of tugsten transferred from the electrode to the surface of the parent metal or solidified weld metal.

e. Torn surface: Surface damage due to the removal by fracture of temporary welded attachments. 
f. Grinding mark: Local damage due to grinding.

g. Chipping mark: Local damage due to the use of a chisel or other tools.

h. Underflushing: Reduction in thickness of the metal due to excessive grinding.

Weld discontinuities may also be divided into three broad classifications: (i) design related (ii) welding process related and (iii) metallurgical. Disign-related discontinuities include problems with design or structural details, choice of the wrong type of weld joint for a given application or undesirable changes in cross section. Discontinuities resulting from the welding process include undercut, slag, oxide and tungsten inclusions, porosity, shrinkage voids, lack of fusion and penetration, craters melt-through, spatter, arc strikes, underfill etc. Metallurgical discontinuities include cracks, fissures, fisheye and segregation or concentration of impurities or alloying elements which arise during the solidification of the weld metal. In decreasing order of harmfulness, weld discontinuities may be classified as (i) crack or crack-like (ii) geometric (iii) lack of fusion and penetration (iv) inclusions and ( $v$ ) porosity.

\subsubsection{1: Crack or crack-like discontinuities}

Cracks are the most serious type of weld discontinuity because they can greatly reduce the strength of weldments. The various types of cracks include microfissures, hot tearing, lamellar tearing, hydrogen-induced cold cracking, stress corrosion cracking and graphitization.

In general, both mechanical and metallurgical factors contribute to the formation of cracks during welding. The formation of crack in a weldment requires:

1. That the material comprising the weldment exhibit a phase or a combination of phases which possess a limited capacity to tolerate strain within some critical range of temperature.

2. That the strain imposed upon the weldment by the combined action of restraint and thermal stresses within this critical range of temperature exceed the strain tolerance of 
the composite microstructure. In general, the greater the tensile strains imposed upon the tail of the weld during solidification, the more severe is the cracking. It should also be noted that welding involves a moving molten solidifying zone and that the weld thermal strains are cumulative.

The first factor is of metallurgical origin and is influenced by the constitution of the alloy, the thermal and mechanical processing variables, the welding parameters used, and the geometrical features of the weld which affect the temperature distribution in the weld and the surrounding heat affected zone. The second factor is strictly mechanical, although the volume changes accompanying metallurgical phase transformations contribute to the overall strain pattern in the weldment.

The various kinds of cracking described next are primarily based on the Metals Handbook [9].

Microfissures: Microfissures are metallurgically related flaws formed during weld metal solidification. Microfissures can be of the hot or cold type of cracking, induced by a small strain. Their detection is difficult by conventional nondestructive techniques; usually metallographic preparation is necessary. Applied strain is needed to produce cracking; the amount needed to initiate cracking depends on material toughness. Because material is always subject to some type of strain, either from external or internal sources, the occurrence of cracking is therefore controlled by the conditions of welding.

Hot Cracking: Hot cracking is the most common of the weld metal and heat affected zone cracking. These cracks result from internal stress developed on cooling following solidification. The term encompasses cracks that form while a weld is solidifying or when a weldment is reheated. Solidification cracking, liquation cracking, ductility-dip cracking, and strain age cracking or reheat cracking are types of hot cracking. The cracks are normally intergranular in nature and can occur in either the weld metal or the HAZ.

Solidification Cracking: Solidification cracking occurs within a few hundred degrees of the nominal liquidus temperature of the weld metal and is induced by welding stresses 
and the presence of low melting point constituents that form as a result of segregation during the liquid-to-solid-phase transformation process.

Liquation Cracking: Liquation cracking (often called hot tearing or HAZ burning) is another type of high-temperature cracking that may occur in the welds of all metals. Localized liquation of low-melting-point constituents such as foreign inclusions, carbide phases, or localized segregations of certain alloying elements in the solid may form liquid grain boundary films. The attendent welding strains may, in turn, be sufficiently high to open liquation cracks. Liquation cracking may also occur in the partially melted zone by the liquation of low melting inclusions or second phase segregates and by grain boundary melting. The width of the partially melted zone depends on the nature of segregates and the thermal gradient perpendicular to the weld interface.

As will be seen in a subsequent section, microfissures, hot cracks, solidification cracks and liquation cracks occur by the same mechanism. Hot cracks are large macroscopic tears and can be formed as a result of solidification cracking, liquation cracking or strain age cracking (cracking during post weld heat treatment). Similarly, microfissures can be formed due to solidification cracking, liquation cracking or strain age cracking. The only difference between the two i.e., hot cracks and microfissures, is with regards to the dimensions of the crack. Furthermore, microfissures formed during welding get further extended into hot cracks during post weld heat treatment. In further discussions of weldability testing, theories of solidification cracking and HAZ cracking, the terms microfissures, hot cracks and hot tears are used synonymously as they are all closely related and the same mechanism governs their formation during welding. A clear cut distinction based on the mechanism of formation exists only between cracks formed during welding and cracks formed during post weld heat treatment.

Ductility-dip Cracking: Many alloys, including cupronickels, austenitic chromium-nickel steels, nickel-based alloys and aluminum bronzes exhibit a temperature range over which ductility and tensile strength drop sharply. Above and below the ductility-dip embrittle- 
ment range, fracture occurs by microvoid coalescence resulting in high values of strength and ductility. Within the embrittlement range, fracture is intergranular and brittle. Such intergranular failure is normally attributed to the segregation of impurity atoms $(S$ and $P$ in case of austenitic stainless steels) to the grain boundary. These alloys are susceptible to HAZ and weld metal cracking when welded under excessive restraint. Ductility-dip cracking may occur in either the weld metal or the HAZ. This type of crack forms while the weld cools through a range of temperatures where the ductility of the particular alloy is inherently low. If sufficient strain is present as the metal cools through the ductility-dip temperature range, cracking occurs. In some cases, cracking may be initiated by other forms of hot cracking followed by ductility-dip cracking when the segregating constituents not only embrittle grain boundary regions in the ductility-dip temperature range but also substantially depress the melting temperature at the grain boundaries.

Reheat Cracking or Stress-relief Cracking: Reheat cracking is thought to be closely related to the phenomenon of creep rupture. This kind of cracking is also called strain age cracking $(S A C)$ or post weld heat treatment cracking and may form in the HAZ of welds. Post weld heat treatment may be specified for weldments to reduce (or eliminate) residual stresses and/or to restore toughness in the $\mathrm{HAZ}$ and weld metal. Reheat cracking may take place during this post weld heat treatment as a result of the pre-post weld heat treatment microstructure of the HAZ brought about by the welding. Welding heat input induces an elevated temperature solution treatment in the HAZ. During post weld heat treatment the grains may be strengthened sufficiently due to precipitation so that relaxation of internal stresses may occur only by grain boundary sliding, with little or no grain deformation; hence, reheat cracking occurs. Reheat cracking has also been linked to temper embrittlement. Austenitic stainless steels, ferritic creep resisting steels, microalloyed steels and some nickel-base alloys are susceptible to reheat cracking.

Hydrogen Induced Cold Cracking: Cold cracking is the term used for cracks that occur after the weld has solidified and cooled; it can occur in either the HAZ or the weld metal 
of low-alloy and other hardenable steels. Because these cracks occur under conditions of restraint, they are are often referred to as restraint cracks. Cracking may occur several hours or days after the weld has cooled; consequently, the term "delayed cracking" is also used. On the basis of location, cracks are often described as toe cracking, root cracking or underbead cracking. Factors required for cold cracking to occur in steels are: (i) a crack sensitive microstructure i.e the $H A Z$ or weld metal that is susceptible to hydrogen embrittlement. (ii) sufficient hydrogen concentration in the weld (iii) rigid tensile restraint and (iv) a temperature between approximately $300 \mathrm{~F}$ to $-150 \mathrm{~F}$.

Lamellar Tearing: Lamellar tearing is a form of base-metal cold cracking that occurs in steels parallel to the plate surface adjacent to the welds. Lamellar tearing is associated with non-metallic inclusions such as oxides, sulfides, and silicates that are elongated in the direction of rolling. The net result of these inclusions is a decrease in the throughthickness ductility. Lamellar tearing in restrainted weld joints results from inadequate ductility in the through-thickness direction.

Fisheye: Fisheyes are cracks surrounding porosity or slag in the microstructure of a weld. Fisheyes are a form of hydrogen embrittlement cracking caused by the presence of hydrogen that collects at these locations and that embrittles the surrounding metal in the same way cold cracking does. What distinguishes fisheye is a characteristically shiny appearance when viewed under an optical microscope. Fisheyes also termed as "flakes", "blisters", and "halos", are predominantly found in ferritic steels welded with high hydrogen electrodes. The overall fracture appearance of fisheyes usually indicates a mixed or quasicleavage fracture mode. When a halo is examined at high magnification, a fracture surface with a pattern radiating from a nucleating discontinuity is revealed. Small, buried microfissures also have been noted to nucleate fisheyes in as-welded E6010 weld metal.

Ferrite Vein Cracking: Another form of hydrogen induced cracking has been observed in electroslag weld metal. Unlike cold cracking of high-strength steels, ferrite vein cracking 
occurs along the grain boundary ferrite in low carbon steels that were believed immune to hydrogen induced cracking. A typical vein crack is usually less than a quarter inch long and always occurs along the grain boundary ferrite.

Stress Corrosion Cracking: This type of cracking takes place when hot concentrated caustic solutions are in contact with the steel that is stressed in tension to a relatively high level. The high level of tension stress can be created by loading or by high residual stresses. Stress corrosion cracking will occur if the concentration of the caustic solution in contact with the steel as well as if the stress level in the weldment is sufficiently high. Graphitization: This type of cracking is caused by long service life exposure to thermal cycling, or repeated heating and cooling. This may cause a breakdown of carbides in the steel into small areas of graphite and iron. When these graphite formations are exposed to thermal cycling, cracking occurs. It will more often occur in carbon steels deoxidized with aluminum. The addition of molybdenum to steel restricts graphitization.

\subsection{4: Weldability}

The general definition of weldability as given in the welding handbook [7] taking into consideration all the welding processes, and taking note of all the different types of construction, whatever properties they may have is:

"A metallic substance is considered to be weldable to a stated degree by a given process for a given purpose, when metallic continuity can be obtained by welding using a suitable procedure, so that the joints comply with the requirements specified in regard to both their local properties and their influence on the constuction of which they form part of".

When considering weldability and weld defects, two general types are of concern: fabrication weldability and service weldability. From the fabrication weldability view point, hot, cold and stress relief cracking must be avoided along with other more common weld defects which were described above. From the service weldability point of view, the strength, toughness and creep resistance of the weld metal and the heat affected zone 
must match that of the base metal. Further discussion in the present dissertation is however, only limited to the fabrication weldability of materials.

\subsection{5: Weldability Testing}

Attempts to define weldability as an inherent characteristic of a particular type of alloy invariably meet with failure as a result of heat-to-heat variations in composition, grain size, impurity level, and other less tangible variables. Of the commonly encountered weld defects, hot cracking appears to be influenced most strongly by material characteristics. Other forms of weld defects such as cold cracking, porosity, slag entrapment, lack of fusion, etc., are influenced more by process and procedure related variables than by the differences between individual heats of material.

The hot cracking susceptibility of an alloy is dependent upon its elemental composition and of the distribution of these elements within the material. Thus even if two heats of the material were to be produced with the same overall composition, these materials would not necessarily exhibit similar hot cracking tendencies because of the variations in the solute distribution. This distribution is dependent upon the entire past history of the material, from the time an alloy was cast as an ingot, to the time that the welding is actually performed. Thus, it is futile to attempt to assign a meaningful cracking susceptibility index to a particular alloy. However, one may attempt to explain the heat-to-heat variations for a given alloy, and eventual conclusion may be drawn as to how to control the hot cracking susceptibility of the alloy. In the past couple of decades, weldability tests have been developed to assess the hot cracking susceptibility of a material to welding procedures and microstructural conditions.

Under normal conditions, weld metal hot cracking is produced only within a relatively narrow region directly behind the instantaneous position of the solid-liquid interface at the trailing edge of the weld pool. Microfissuring in the weld heat affected zone is normally observed only in the region of the heat affected zone at either side of the location 
of the weld pool. The following quantitative measurements have been used for comparing the weldability of a material under different welding procedures and/or metallurgical conditions.

\subsubsection{1: Quantitative Measures of Hot Cracking}

Maximum Crack Length (MCL): Since the crack length is proportional to the width of the cracking temperature range for a given welding procedure and microstructural condition, the maximum crack length has been observed to provide a useful qualitative index of the cracking sensitivity. Measurements of maximum crack length can be used as a rapid screening technique during preliminary testing of materials provided a constant weld pool geometry is maintained.

The Total Combined Crack Length (TCL): The total combined crack length produced in the weld deposit by a given combination of welding procedure and microstructural condition has proved to be the best quantitative index of the cracking sensitivity of the weld metal. Similarly, the total combined crack length for heat affected zone cracks provides a quatitative index of the base metal cracking sensitivity for a given welding procedure and microstructural condition.

The average crack length (the length of the average crack in a particular specimen obtained by dividing the TCL by the total number of observed cracks) and total number of cracks are also used as indices to compare the welding susceptibility of a material. It should be noted that the above measurements are useful only for a given constant heat input. When comparing welds made with different heat inputs, the data is normalized by taking into account the area of the HAZ. Other quantitative measurements obtained by simulated tests that are used in discriminating cracking susceptibilities are nil ductility temperature (NDT), the temperature range between nil strength temperature (NST) and the ductility recovery temperature (DRT). NDT, NST and DRT are obtained from gleeble hot ductility testing and is discussed further in a subsequent section. 


\subsubsection{2: The Varestraint Test ${ }^{[10]}$}

An ideal weldability test should incorporate [11]:

1. Ability to show a direct correlation with the actual fabrication and service behavior

2. Reproducibility of results with freedom from variation due to human element

3. Sensitivity to small changes in a test variable

4. Ability to show the effects of several testing variables

5. Economical preparation of specimens and running of a test

6. Applicability to all welding processes

Over thirty different cracking tests have been developed prior to the development of the Varestraint test but none of them possessed all the above mentioned features.

The varestraint (VAriable RESTRAINT) test permits the evaluation of base metal weldability as well as determining the influence of the particular welding process and the associated welding variable on hot cracking. The varestraint test provides a reproducible means for augmenting the normal shrinkage strains and thus simulates the large shrinkage strains characteristic of a highly restrained production weldment in a small laboratory specimen. The degree of restraint is simulated by the application of a controlled augmented strain to the specimen during welding.

The varestraint testing procedure utilizes a small, laboratory scale specimen supported as a cantilever beam as shown schematically in fig 2.4(a) [10]. A weld is deposited from left to right, as indicated, using any combination of process, joint geometry, welding parameters and microstructural conditions of interest. As the arc passes the point marked $A$ in the fig 2.4(a), a massive, pneumatically actuated loading yoke bends the specimen downward suddenly to conform to the radius of curvature of the top surface of the removable die block, B. Meanwhile, the arc traverses steadily onward and is subsequently interrupted in the run-off area at $C$.

Since the specimen tends to bend preferentially at the location where the heat of 

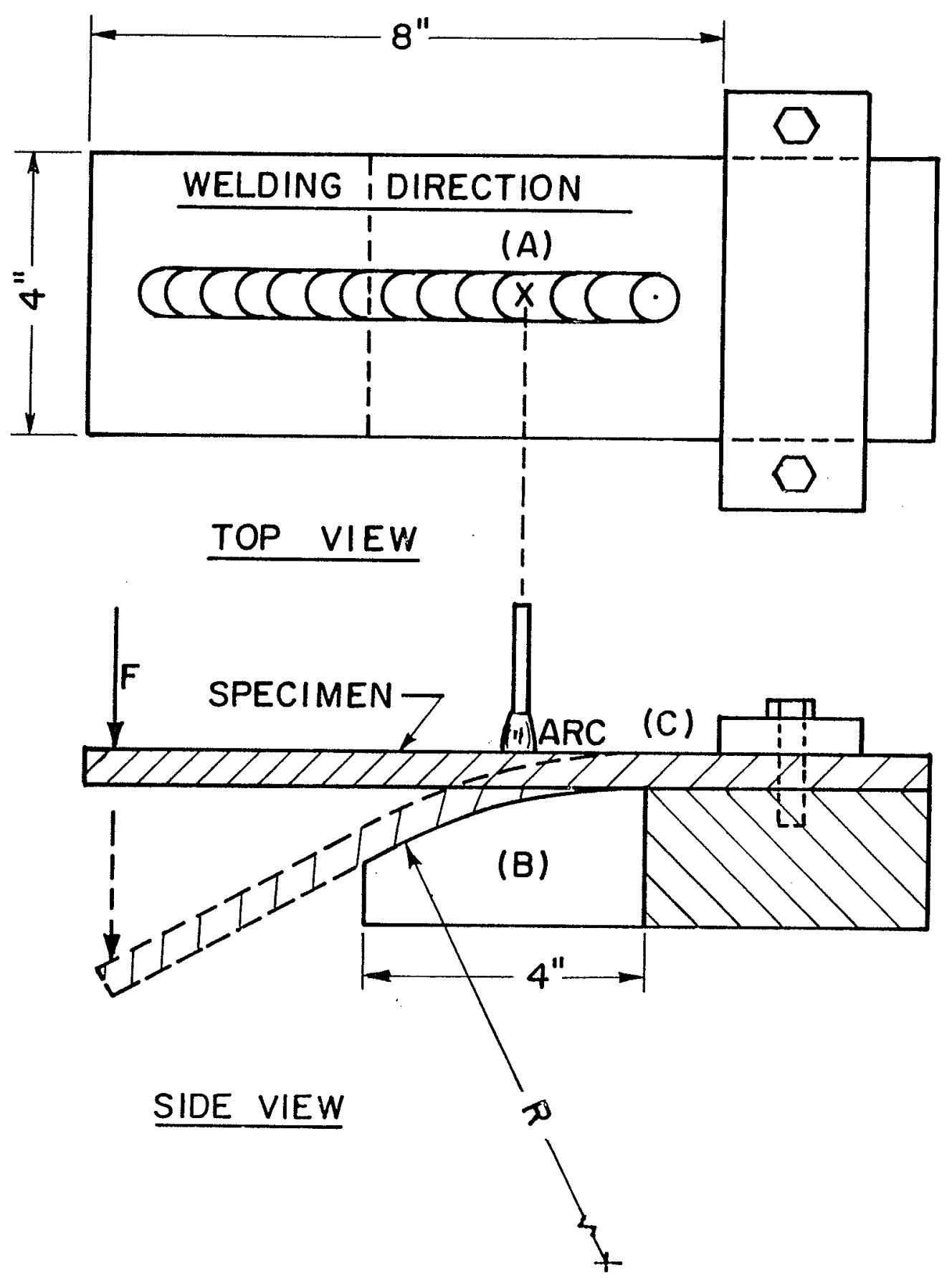

Fig. 2.4 (a): Simplified sketch of the operation of Varestraint testing device [10]. 
the arc has reduced the strength of the material to a minimum, the addition of auxiliary bending plates as shown in fig 2.4(a) forces the specimen to conform to the contour of the die block. From the geometry of the system, the nominal value of the suddenly applied augmented tangential strain, $\epsilon_{t}$, in the outer fibers of the test specimen can be calculated as

$$
\epsilon_{t}=\frac{t}{2 R}
$$

where $t$ is the specimen thickness and $R$ is the radius of curvature of the die block.

Therefore, by substituting a die block with the appropriate radius of curvature, any desired augmented strain can be applied to the weldment at any pre-determined instant during the welding deposition. Since the magnitude of the total augmented tangential strain is independent of the welding parameters, the effect of welding process, weld composition, and other parameters influencing the microstructural features of the weldment, these parameters can be isolated from the mechanical effect of the externally imposed restraint. The suddenly applied augmented strain is imposed over a four inch portion of the specimen parallel to the welding direction with the weld pool located at one end of the test section. Thus, the weld pool, together with the contiguous four inch length of the weld bead and the associated heat affected zone are subjected to a reproducible augmented strain in the presence of the temperature distribution characteristic of the particular welding procedure employed.

Since the magnitude of the augmented strain is independent of the welding conditions, the characteristic shape of the weld pool and the temperature distribution surrounding the weld can be altered by appropriate choice of the welding variables. For a particular weld pool geometry and temperature distribution, cracking occurs in those regions where the temperature of the material at the instant of straining is within the crack sensitive range and the augmented strain exceeds the strain tolerance of the existing weld microstructure. Furthermore, the effect of initial microstructural condition on hot cracking can be 
evaluated from the varestraint test as the primary influence of microstructure is to alter the crack sensitive temperature range of the material during welding. The hot cracking produced by the varestraint test is invariably adjacent to, and usually contiguous with, the location of the weld pool at the instant of application of the augmented strain, fig 2.4(b).

The inherent restraint provided by a simple rectangular block is generally too low to cause cracking in the absence of augmented strain. Hence, the minimum augmented strain required to cause cracking with a given set of welding parameters and microstructural condition provides a quantitative index of cracking sensitivity, called the cracking threshold. In addition, the variation of the cracking threshold produced by changes in the welding process and the welding parameters provides a quantitative method for comparing welding procedures. By varestraint test, cracking can be induced in any material with a suitable choice of the augmented strain. This is one of the major advantages of the test since it permits assigning even a crack insensitive material to its proper relative position on the crack sensitivity scale. In addition, the correlation of the results of the varestraint tests with the known fabrication and service behavior is excellent. Because of these reasons, the varestraint test is the most widely used and accepted weldability test for assessing the welding susceptibility of materials. The varestraint technique has been shown to be of proven value in [12]:

1. The evaluation of the relative hot cracking sensitivity of different types of base matals.

2. The evaluation of the heat-to-heat variations in hot cracking susceptibility.

3. The evaluation of the hot cracking sensitivity of filler metals as deposited and modified by the welding process.

4. The evaluation of the effect of specific alloying elements on the hot cracking susceptibility of base metals.

5. The determination of hot cracking temperature range.

6. The performance of fundamental studies on the mechanisms of hot cracking. 


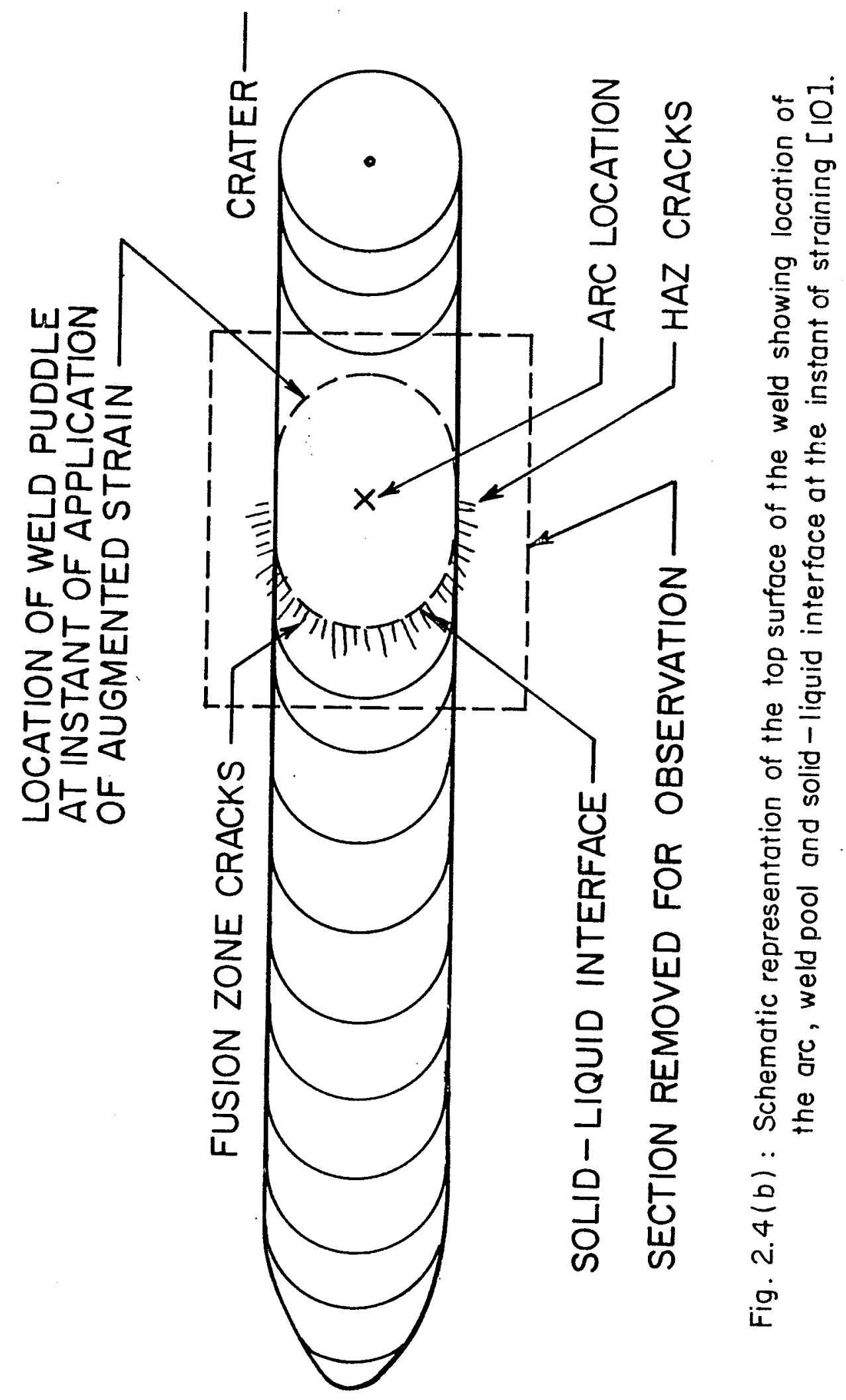


Because of the above reasons, the varestraint test is the most widely used and accepted weldability test for assessing the welding susceptibility of materials.

\subsubsection{2: Gleeble Hot Ductility Testing}

"Gleeble" is an equipment that was developed [13,14] for measuring the effects of testing temperature and prior thermal history on the hot ductility of materials. Gleeble reproduces in a cylindrical test bar the time-temperature cycle to which the metal adjacent to the weld is subjected to during the welding cycle. At any selected instant during the time-temperature cycle, a tensile load simulating the shrinkage stress imposed by the weld, can be applied rapidly to fracture the specimen. Instrumentation is provided to record the thermal cycle which the specimen had undergone, the ultimate tensile load required to fracture it, and the amount of elongation which it underwent. Subsequently, the reduction of the area of the broken specimen is also measured. These data obtained by testing a series of specimens at various points in the time-temperature cycle provide the basis for evaluating the susceptibility of the material to hot cracking in the weld heat affected zone.

The hot ductility response of many metals during the heating portion of the thermal cycle appears to have several features in common; these are:

(i) Rapid loss of ductility with the onset of intergranular fracture.

(ii) A nil ductility temperature (NDT) defined as the temperature at which the material no longer exhibits any ductility. NDT is a characteristic property of a material.

(iii) A nil strength temperature (NST), where the material exhibits no strength at all, generally corresponding to the metals bulk solidus temperature. Another important parameter obtained from the on cooling curve is the ductility recovery temperature (DRT). DRT is the temperature on-cooling from above NDT or NST at which some ductility of material is apparent.

Interpretation of hot ductility results has typically been based on the reduction of area exhibited by specimens tested at various temperatures during both the heating and 
cooling portions of the thermal cycle. The performance of the specimens tested on cooling from the nil ductility temperature has been the primary criterion on which predictions of susceptibility to HAZ cracking during welding have been based. Fig 2.5 [15] shows the typical behavior of austenitic alloys considered to have good resistance to cracking. This behavior is characterized by excellent ductility tested on heating close to NDT and when tested on cooling from NDT, the ductility is essentially comparable to that obtained on heating i.e., the weld thermal excursion has no adverse effects on ductility of such alloys and that their good ductility will prevent them from cracking during welding.

The typical behavior of alloys considered to be highly susceptible to cracking is illustrated in fig 2.6 [15]. Materials in this class may exhibit almost as good ductility when tested on heating as the crack resistant alloys. A marked difference is evident, however, during testing on cooling from NDT. Unlike the crack resistant alloys, materials considered to have poor resistance to cracking have poor ductility on cooling from their NDT. Their ductility on cooling may stay at the nil ductility level or they may show only moderate recovery. It has been considered that this loss of ductility which would occur in the regions of HAZ close to the weld fusion line causes them to be highly susceptible to cracking during welding.

In the past, experimental methodologies utilized in Gleeble hot ductility testing and data interpretation have been the subject of considerable investigations. Relating the ductility recovery temperature on cooling from elevated temperatures to $H A Z$ liquation cracking was proposed by Nippes et. al. [13.,14]. Owczarski et. al. [13,14,16] suggested that testing on cooling from a peak temperature from above the NST was more appropriate in terms of discriminating differences in cracking susceptibility between materials. They stated that the extent of the nil ductility region in the HAZ and the amount and rate of recovery of ductility on cooling correlated with the degree of cracking sensitivity. A wider nil ductility region or slower recovery of ductility was related to a higher susceptibility to cracking. Duvall and Owczarski [1] have also suggested that the temperature range 
between the NST and DRT is of primary concern. Muesch [17] proposed a cracking factor, (NST - DRT)/NST, based on the temperature range between NST and DRT. Donati et. al. [18] used this temperature range to predict the cracking tendancy.

Kreischer [15] and Williams [19] have suggested that the measurement of hot tensile strength was equally important as measuring ductility. Kreischer [15] has observed that crack sensitivity of certain alloys (type 347 stainless steels, forged N-155, medium carbon iron-base austenitic alloy containing $8 \%$ minimum $\mathrm{Ni}, 17 \%$ minimum $\mathrm{Ni}$ plus $\mathrm{Mn}$ and $3 \% \mathrm{Cr}$, Inconel $\mathrm{X}$ ) as determined by hot ductility tests did not correlate with the actual cracking propensity in the welds. His observations on hot tensile strength of these alloys suggested that recovery of tensile strength as well as ductility might have a significant effect on the tendancy of a material to develop hot cracks during welding. The possible influence of tensile strength on weld cracking is related to the condition that thermal strains developed during welding due to the heat introduced by the welding arc and then from the solidification and cooling, can be absorbed as both elastic and plastic strains in the welded joint.

Following Kreischer, consider two hypothetical materials whose stress-strain characteristics at elevated temperature are shown schematically in fig 2.7 [15]. Fig 2.7 indicates that only a small portion of thermal strains generated during welding can be accommodated elastically in a weaker material. The remainder must occur as plastic strain, or the material will crack if it has insufficient plastic-strain capacity. Thus, a comparatively weak material would have to exhibit good ductility in the hot ductility test if it is to be crack resistant. On the other hand, the stronger material can accommodate most of the strain elastically. It would therefore only have to have little plastic-strain capacity to accommodate the rest of the strain without cracking. Thus a material with high tensile strength as measured in the hot ductility test might well be resistant to cracking even if it has low ductility. If the welded joint of a material cools without causing the HAZ to be plastically strained, then it should not be necessary for that material to possess good 


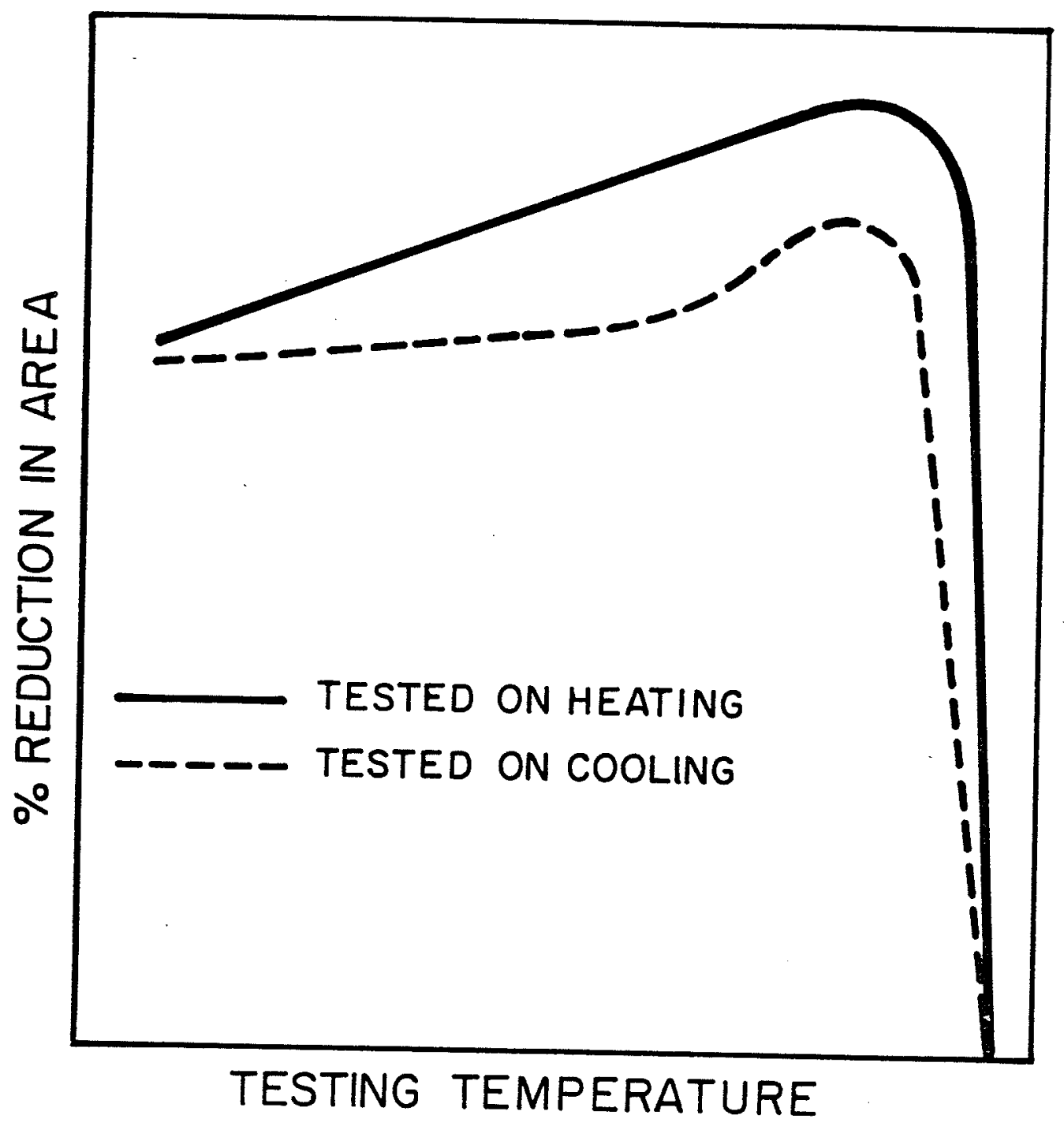

Fig. 2.5: Schematic representotion of the typical behovior of highly crock resistant moterials [15]. 


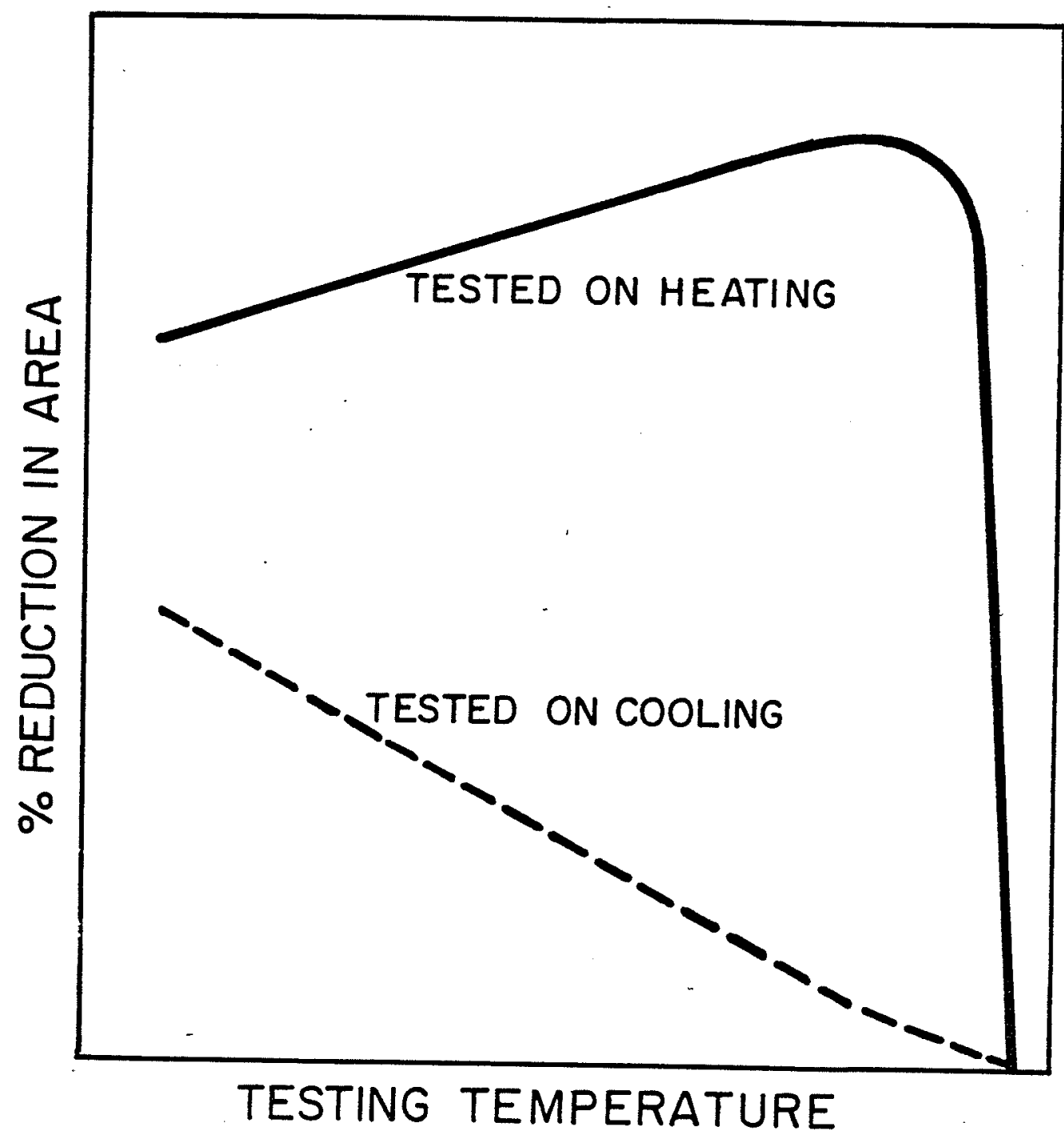

Fig. 2.6: Schematic representation of the typical behovior of highly crock sensitive moterials [15]. 


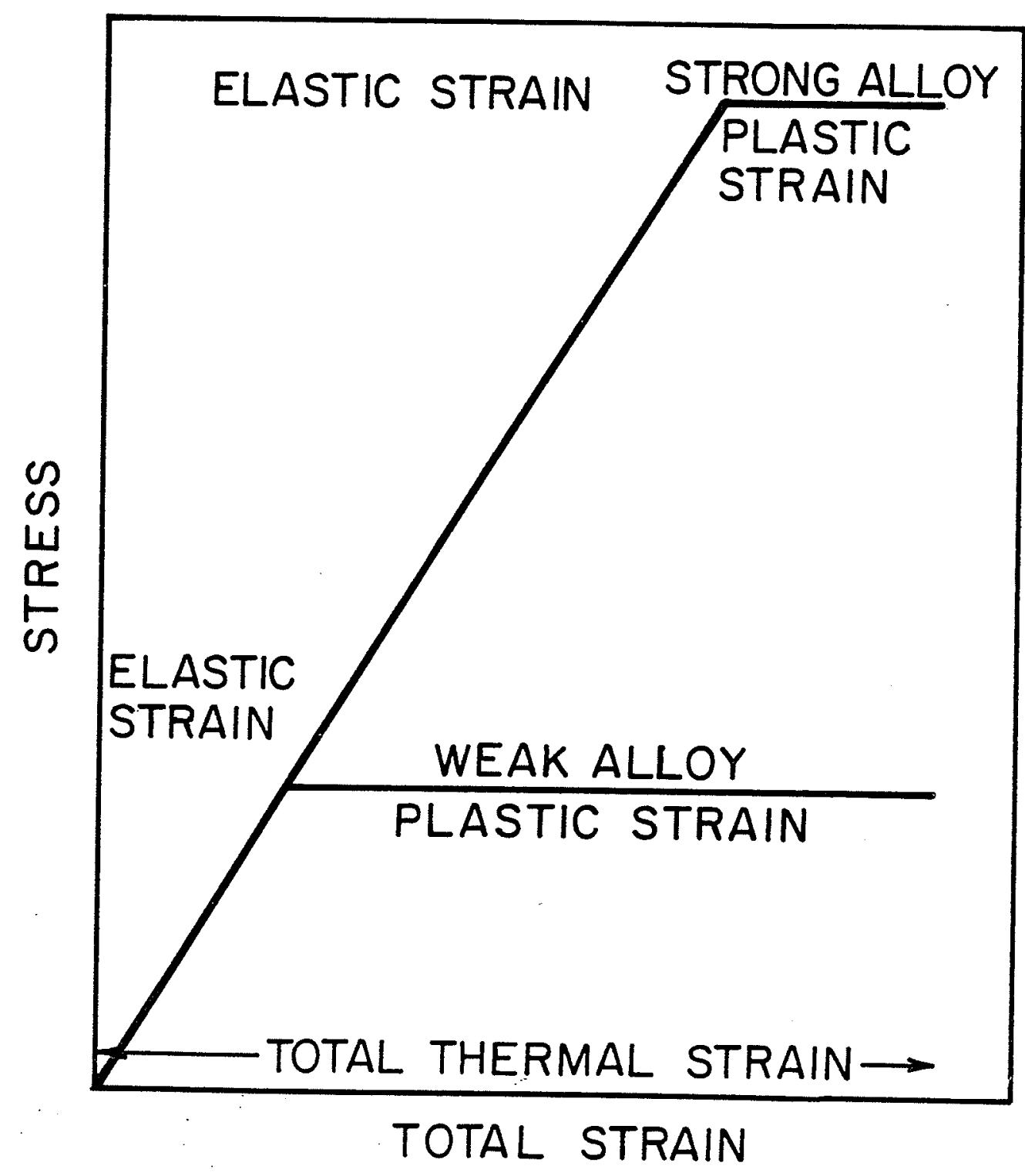

Fig. 2.7 : Schematic representation of thermally produced strain $[15]$. 
ductility to be crack resistant.

Kreischer's analysis thus indicates that tensile strength values measured in the hot ductility test might be equally as important for accurate prediction of crack susceptibility as the reduction of area. According to his postulation, the poorer the ductility of a material, the higher its tensile strength recovery would have to be for it to be crack resistant. Kreischer [12], based on experimental observations, also suggested that in cases where the nil ductility and nil strength temperature are different, tests should be conducted on cooling from both these temperatures. This was based on the assumption that the nil strength temperature of a material will be approximately equal to its solidus temperature and will thus correspond to the region of HAZ just adjacent to the fusion line. If the material has a wide temperature range between its NDT and NST and testing is not done to and from the NST, then the behavior of a significant portion of the HAZ will not be known. Thus results based solely on heating and cooling from NDT are suspect because the properties of the untested portion of HAZ which reached peak temperatures between NDT and NST might be very poor. These views are supported in retrospect by the results of Owczarski et.al. [1] who have noted that tests conducted on cooling from and above the NST produced more meaningful indications of relative crack sensitivity than did tests from NDT.

Another aspect of hot ductility results that is not well understood is the significance of the temperature range between the NDT and NST. In many materials, loss of ductility and strength occurs at the same temperature.In others ductility may drop to zero a hundred or more degrees below the temperature at which the tensile strength is negligible. Both crack resistant and crack sensitive materials have been found in each of the two categories. However, it seems likely that materials which recover strength and ductility simultaneaously (i.e., they have a common NDT and NST) should be more crack resistant than ones which do not begin to recover any ductility until they have cooled well below their nil-strength temperature. 
However, the present status of the gleeble hot ductility testing is such that results cannot be interpreted with a satisfactory degree of reliability. A significant amount of work needs to be done to provide the desired understanding of the significance and interpretation of hot ductility results. Despite the aforementioned disadvantage, the gleeble hot ductility testing provides simulated weld and heat affected zones which are imperative for a complete microstructural characterization of the HAZ. It should also be noted that both varestraint testing and gleeble hot ductility testing results are best suited for studying crack sensitivity during welding (fabrication weldability) as opposed to correlating crack sensitivity during service (service weldability). Other tests have to be used for studying service weldability.

\subsection{6: Metallurgical Mechanisms of Formation of Weld Defects}

It has been known from a long time [20] that the HAZ type of hot cracking is developed as as result of the liquation of grain boundary segregates in the region which borders the fusion line and the weld. The region of the HAZ within which liquation occurs is limited to the partially melted zone and thus, hot cracking or cracking resulting from liquation is indigenous to the partially melted zone. Since the term hot cracking has been generally used in the welding literature to describe high temperature liquation cracking in both the weld metal and HAZ, it's use is often ambiguous. As a consequence, in recent literature [21], the terms 'weld solidification cracking' and 'HAZ liquation cracking' had been adopted to more accurately classify weld metal and $H A Z$ hot cracking respectively. Since, a weld zone may be considered as a small casting, often a continuous casting formed under special conditions [1]: an intensely stirred liquid pool, high temperature gradients, rapid growth rates, intimate contact between the molten alloy and the mold including partial melting of the mold, the development of hot cracks in the weld, i.e. solidification cracks, depends on the same factors which determine the hot tearing of castings [22,23].

\subsubsection{1: Solidification Cracking in Welds}


Solidification cracks are those which have been wholly or almost wholly initiated and propagated above the solidus or slightly below the solidus whilst the alloy is solidifying from the molten state. Hence these cracks occur in the fusion zone of the weld. It should be noted that some authors use the term 'subsolidus cracking' if the cracks were to form below the solidus. However, as will be seen in the subsequent discussion on various theories of solidification cracking, no consensus exists as to whether the cracks initiate above the solidus or slightly below the solidus. There exists some evidence in support of all the theories.

The main factors causing solidification cracking are mechanical and metallurgical. Mechanical factors include thermal strains caused by the dynamic expansion and contraction of the weld and heat affected zones, the thickness and size of the adjoining material, weld joint design and the size and shape of the weld pool. In general, the greater the tensile strains imposed upon the 'tail' of the weld during solidification, the more severe the cracking. Principal metallurgical factors are the presence of a liquid phase at the interdendritic and/or grain boundaries during a specific temperature interval of freezing and the accompanying solidification structures. Significantly, the precise characteristics of the thermal strains, weld pool shape and solidification structures depend mainly on the temperature distribution created by the act of welding and changes in these patterns may be brought about by changes in the welding parameters.

\subsubsection{1: Review of Theories of Solidification Cracking}

Three different theories have been advanced to explain the solidification cracking of weldments. They are (i) shrinkage-brittleness theory [22] (ii) strain theory of hot tearing [20] and (iii) generalized theory of cracking [23-25] In the shrinkage-brittleness [22] theory

it is argued that the basic cause of cracking is the inability of the semi-rigid network of interlocked dendrites to withstand the shrinkage strains imposed upon it during cooling through a 'brittle temperature range'. Protagonists of the strain theory of hot cracking 
[20] believe that cracks are formed during the last moments of solidification when a liquid film stage is reached due to seperation of a solid-liquid mass in a region where solid-solid bridges have not been established. The generalized theory according to its protagonist, Borland [23-25], attempts to reconcile, modify and extend the above two theories and to explain how the liquid quantity and distribution during freezing affects the cracking tendency. In particular, it was suggested by Borland that the distribution of liquid is largely influenced by the interphase (solid-liquid) and intercrystalline boundary energies. The generalized theory suggests that for cracking to occur it is not a sufficient condition that a wide freezing range existed but liquid should also be present over a relatively wide temperature interval in a form that allows high stresses to be built up between grains. A high ratio of the solid-liquid to solid-solid boundary energy tends to confine the remaining liquid to the grain edges and the grain corners and thus greatly reduces hot cracking. The three theories are discussed in detail in the following sections based on the original contributions.

\subsubsection{1(a): Shrinkage-Brittleness Theory ${ }^{[22]}$}

The origins of shrinkage-brittleness theory result from numerous investigations of hot cracking (tearing) tendencies of aluminum alloys but the ideas developed are equally applicable to other alloy systems. Shrinkage-brittleness theory takes the view that cracking occurs in the brittle temperature range (B.T.R). According to the Russian investigators [23-25], the upper temperature limit of the B.T.R is defined by the "solidifying stages when the dendrites come into contact with each other and interlock thus preventing the liquid phase from circulating around them (also called 'coherent temperature') and the lower limit by the temperature at which the strength of the grain boundaries is sufficient enough to absorb the loads imposed upon them. Some investigators consider the lower limit of B.T.R to be the solidus. The Russian view is more precise, but in many practical instances the two definitions can be considered virtually the same. The mechanism of 
cracking is thought to be as follows:

At some time during the liquid-solid stage, the primary dendrites, growing at the expense of the decreasing volume of the liquid, come into contact for the first time (coherent temperature) and interlock, thereby forming a coherent network. During subsequent cooling, contraction strains develop, and when the critical rupture stress has been exceeded, a rift will occur in the network. These newly formed fissures will persist if there is insufficient liquid remaining to heal them. Once the alloy has passed safely below the solidus, cracks are unlikely to form, it being assumed that the solid metal is ductile at these temperatures. Cracking therefore is only likely to occur in the brittle range (effective interval of crystallization) i.e., the range of temperature between the coherent temperature and the solidus (see fig 2.8). According to Pumphrey and Jennings [22] the cracking tendency is proportional to the extent of brittle range.

Since the brittle temperature range of an alloy lies within the freezing range, its extent is primarily dependent on the composition of the alloy. However there are other factors whose effects can also be significant. The main factors apart from constitutional factor are the mode of distribution of the liquid phase and the factors which influence the mode of distribution of the liquid phase. The temperature at which a coherent network forms during solidification of an alloy is dependent on the shape of the dendrites, which in turn, is principally dependent on the temperature gradients within the cooling mass. Pumphrey and Jennings have conducted experiments by varying the cooling rates on a series of Al-Si alloys of varying silicon contents and observed that an approximately linear relationship existed between the proportion of eutectic in the alloy of the lowest silicon content which did not crack in a given set of circumstances and the average rate of cooling over the solidification range. It appears that the slower the rate of cooling the higher is the proportion of the eutectic liquid situated at the crystal boundaries, and at very slow rates of cooling all the eutectic is intercrystalline. The influence of the eutectic habit on shrinkagebrittleness depends on the fact that the temperature at which a coherent network forms in 


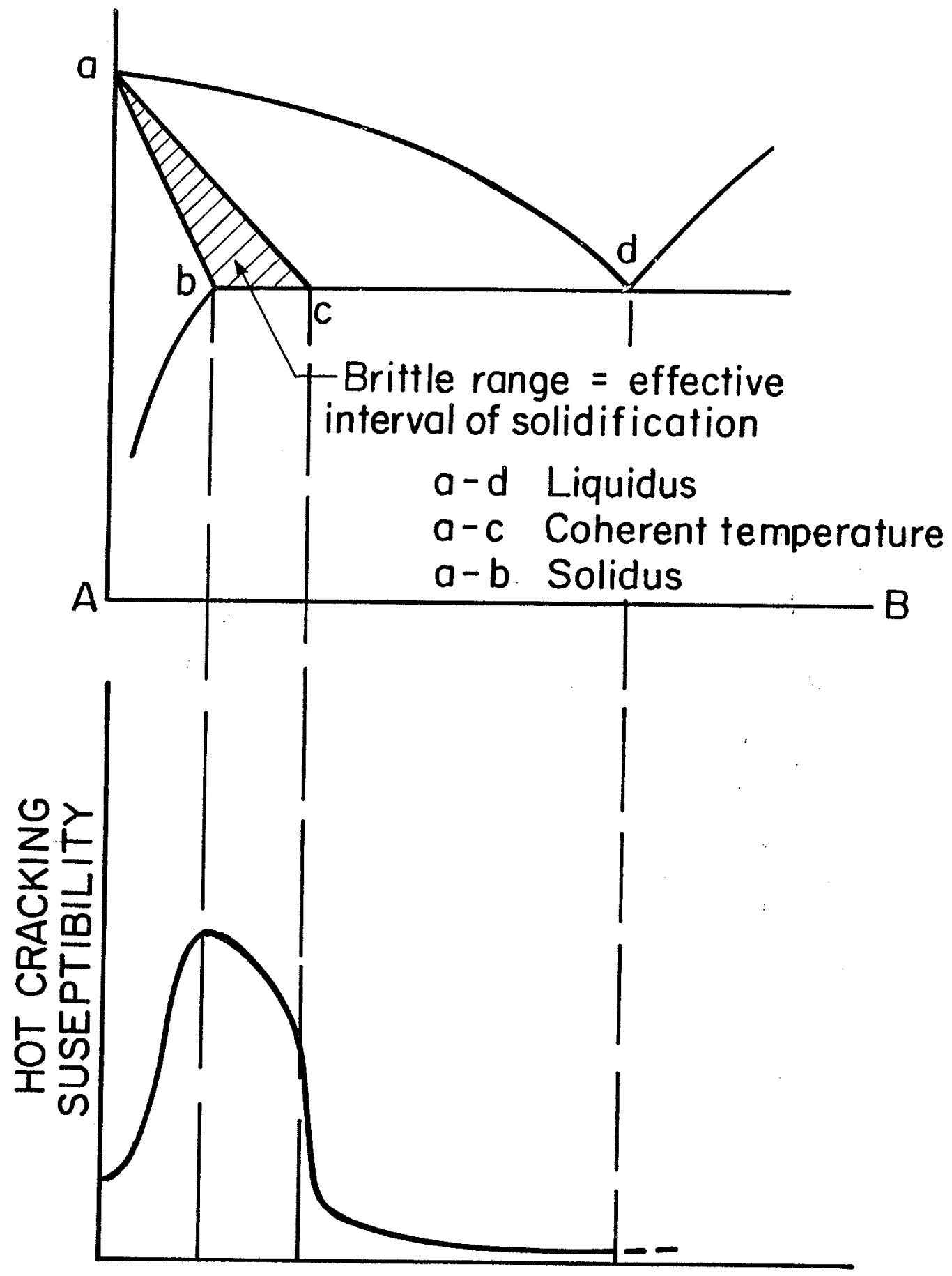

Fig. 2.8 : Hot cracking susceptibility of eutectiferous alloys [23]. 
a solidifying mass is a function of proportion of liquid between the dendrites, as opposed to the liquid between arms of a single dendrite. A minimum shrinkage-brittleness would be expected when there is no tendency for entrapment of liquid, and when, in consequence, all the eutectic in the finally solidified alloy is situated in the crystal boundaries.

The shrinkage-brittleness of an alloy is dependent on the rate of cooling. It has also been observed that the greater the reduction in crystal size (grain size), the more marked is the reduction in cracking. These experimental observations have made Pumphrey and Jennings suggest that the degree to which liquid is entrapped between the dendritic arms during their formation is a function of the linear rate of crystal growth. A reduction in the rate of cooling increases the total time of solidification, and for a given crystal size the rate at which growth takes place from any one of the nucleus is lessened. If, however, the rate of cooling remains constant, an increase in the total number of nuclei in the cooling mass results in a reduced rate of growth for each one. Thus, a decrease in either rate of cooling or crystal size, other things being constant, results in a decrease in the rate of crystal growth, that is, in a decrease in the rate of advancement of the dendritic arms into the surrounding liquid. The slower the rate of dendrite growth the greater is the tendency for the liquid to be concentrated at the tips of the growing dendrites and the smaller the tendency for entrapment between the dendrite arms. Hence, the experimental observation that either a decrease in the rate of cooling or an increase in the number of nuclei in the cooling mass, i.e., a decrease in the crystal size, the greater is the proportion of the liquid situated at the crystal boundaries of any alloy.

The experimental observations of Pumphrey and Jennings led them to the conclusion that during the solidification of an alloy, coherency is gained when the amount of interdendritic liquid (as distinct from liquid entrapped within the dendrites) falls below approximately $5 \%$. If an alloy freezes as a single phase solid solution, there is invariably a brittle range but this is not true of a eutectic alloy. A eutectic alloy, provided that it contains $5 \%$ or more of interdendritic liquid will not gain coherence until the eutectic 
temperature is reached, and consequently will possess no brittle range. This is confirmed by the fact that aluminum binary and ternary alloys show no tendency to cracking when the total alloy content exceeds a certain level. Tensile tests carried on aluminum-silicon alloys have shown that there is, for each alloy, a temperature at which coherence is gained, which is either between the liquidus and the solidus, or coincident with the effective solidus in the relevent conditions.

The concept of 'healing' whereby incipient cracks are considered to be filled with liquid and their ill effects thus overcome, originated from the work of Vero [26]. In order to explain his experimental observations, Vero had postulated that if a solidifying metal mass contains more than a critical volume of eutectic, any cracks formed by the contraction of the primary crystals would be healed by the inflow of eutectic liquid. Pumphrey and Jennings also introduce the concept of 'accommodation' and distinguish it from the concept of 'healing'. 'Accommodation' is the degree to which an alloy is able to withstand shrinkage strains by movement of crystals within the solid-solid mass when the alloy is solidifying in the brittle range thus preventing cracking. Pumphreys and Jennings argue that in welds and small castings, accommodation is more usual than healing, because for healing to occur, there exists the requirement of a critical volume fraction of liquid which may not be present in most cases.

These various ideas were extended by Medovar [27], who states that the variation of hot cracking with alloying additions, for elements forming continuous solid solutions, is determined by the length of the freezing range. If the alloying elements are mutually soluble in either the liquid state or the solid state, then hot cracking should not occur. For systems that do form primary solid solutions, even very small additions of second element will cause severe cracking, owing to the increase in the freezing range, while further additions should tend to reduce cracking. In general, if the freezing range is very short, hot cracking should not occur.

\subsubsection{1(b): Strain Theory of Hot Cracking ${ }^{[20]}$ :}


The strain theory of hot cracking was proposed by Pellini [20] to explain the hot tearing of castings and is equally applicable to the solidification cracking in welds. This is because, as explained earlier, a weld can be considered as a small casting. In order to appreciate the theory it is necessary to understand the concept and significance of the liquid film stage. In passing through the solidus-liquidus temperature range, irrespective of whether the process entails heating or cooling, the alloy develops a condition of essentially continuous liquid films. The strength and ductility of a mass of solid grains separated by liquid films is extremely low. It should be expected that if the grain boundaries contain a continuous network of low melting segregate material, the liquid film should form at the solidus temperature of the segregate material. Fracture during the continuous liquid film stage has been observed to occur in an intercrystalline mode. If the network is not continuous, the fracture occurs at some intermediate temperatures which depends on the amount and distribution of the liquid films. As the composition approaches that of the pure metal the amount of segregate which may be formed is greatly reduced, hence the distribution of the segregate becomes less continuous and less effective in lowering the film stage temperature. The significance of the film stage is that separation can occur through the liquid films which exist either at the true solidus temperature of the metal or in the range of temperatures between the true solidus and the effective solidus temperature of the segregate materials.

Fig 2.9 taken from Pellini [20] illustrates schematically the process of combined hot cracking of weld and HAZ. As the liquid pool behind the arc passes the segregate region, melting occurs between the grains; the lateral distance from the weld which develops melting is determined by the thermal gradients perpendicular to the weld. The top figure illustrates that grain boundary melting occurs to the distance at which the gradient temperature corresponds to the solidus temperature of the segregate film; fusion of the grain occurs at a temperature somewhat higher than the solidus of the grain. At this stage, the $H A Z$ region is in a state of compression due to the thermal expansion of the hot metal 

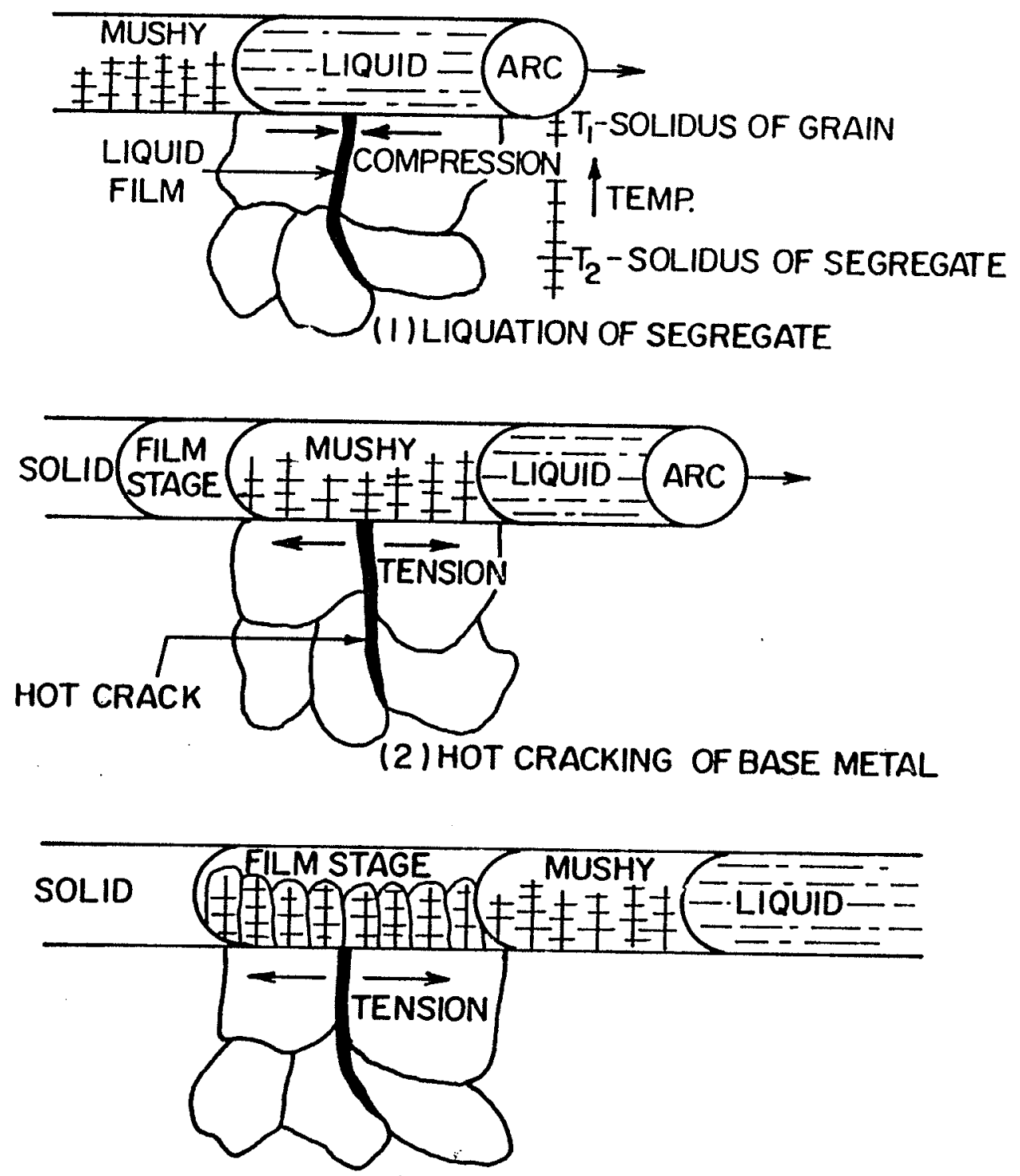

(3) HOT CRACKING OF WELD

Fig. 2.9 : Schemotic illustration of the process of weld hot cracking [20]. 
near the fusion zone. As the arc passes on, cooling ensues with consequent contraction of the $H A Z$, resulting in the development of parting of the film region which produces the $\mathrm{HAZ}$ crack. At this point the weld metal is in a mushy condition and hot tearing is not possible. At a later time the weld metal reaches the film stage and a strain concentration is developed at the HAZ crack position. Thus, the natural shrinkage strain developed by cooling of the weld is concentrated at the HAZ crack point with consequent development of a hot tear in the weld, which is essentially a continuation of the HAZ hot crack. Hot cracks may also occur in the weld alone because of the extension developed in the portion of the weld which is at a film stage by the contraction of colder regions.

It is apparent that film stage fracture is the common element of various manisfestations of hot tear fractures encountered near the complete solidification point of the metals. It should be noted that there is a general agreement in these various manifestations that segregates of lower solidification point than the matrix material tremendously accentuate the probability of hot tearing. Segregated liquid films have been observed to be more effective than the liquid films developed at the true solidus.

Pellini [20] evokes the strain theory of hot tearing to explain this effect. The strain theory provides a generalized mechanism of hot tearing in terms of the time-rate of extension developed in the liquid film stages. According to this theory, liquid film provides the metal a condition which permits hot tearing; however, the actual occurrence of hot tearing is determined by mechanical factors inherent in the rate of extension per unit time imposed on the short time as may be deduced from the rapid drop in liquid film regions. Under nonsegregate conditions, the metal passes through the film stage in short times while under segregate conditions, the liquid film condition exists over a wide temperature range i.e., for much longer times. Accordingly, the rates of extension which do not develop sufficient separation of the liquid film to cause fracture in short times may be fully effective with longer times, for the separation developed in the films is additive with time. Fig 2.10 [20] illustrates the theory schematically. 
It has been assumed by various investigators that hot tears may form while the metal is semi-solid (mushy zone) but that the flow of liquid metal effectively heals the fracture. The strain theory predicts that hot tearing in mushy zone is not possible unless the strain rates are extraordinarily high. The elements of the basic considerations which underlie this prediction are illustrated schematically in fig 2.10 . While the metal is in the mushy zone, which exists at temperatures considerably above the solidus, the extension of the hot zone is distributed relatively uniformly since the interdendritic liquid areas are relatively wide and general flow of pasty mass results. As the hot zone approaches the liquid film stage, the extension of the hot zone is necessarily forced into the narrow liquid films which have no appreciable strength compared to the adjoining solid dendrites. Fig 2.11 [20] illustrates the comparatively high value of unit strains developed in these regions at this time.

The various observations of interrelated effects of metal and mechanical variables led Pellini [20] to a generalization of the hot tearing problem to the following principal elements:

1. The time rate of extension of the hot zone during its film stage is the primary mechanical factor which determines hot tearing. In as much as the film condition stage lasts for only a relatively short fraction of the total solidification time, hot tearing will occur only if a sufficient rate of extension is forced on the film regions during this short time.

2. The rate of extension forced in to the liquid film regions is related to: (a) the overall extent of the hot zone, (b) the amount of the mold restraint, and (c) the length and rapidity with which the adjoining sections cool relative to the hot zone.

3. The presence of elements such as sulfur extends the liquid film condition to lower temperatures and therefore increase the duration of the film stage.

4. The longer the interval of the liquid film stage, due either to the presence of segregates or due to slow cooling, the greater the total amount of seperation developed in the films. Thus,increasing the duration of film stage permits hot tearing to result 

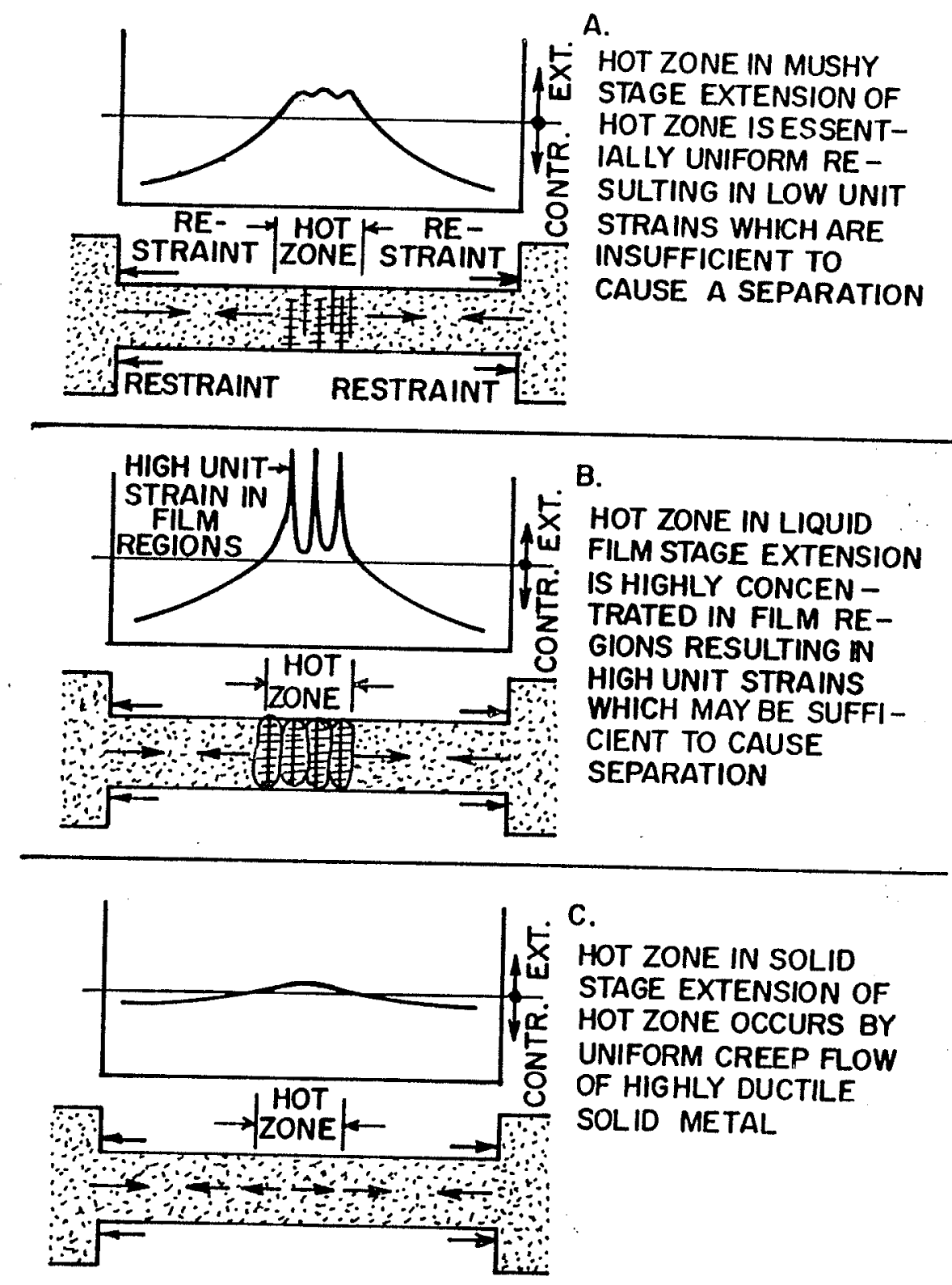

C.

HOT ZONE IN SOLID STAGE EXTENSION OF HOT ZONE OCCURS BY UNIFORM CREEP FLOW OF HIGHLY DUCTILE SOLID METAL.

Fig. 2.10 : Illustrating the nature of strain distribution (extension and contraction ) which exists during various stages of solidification in a casting system containing hot spot [20]. 


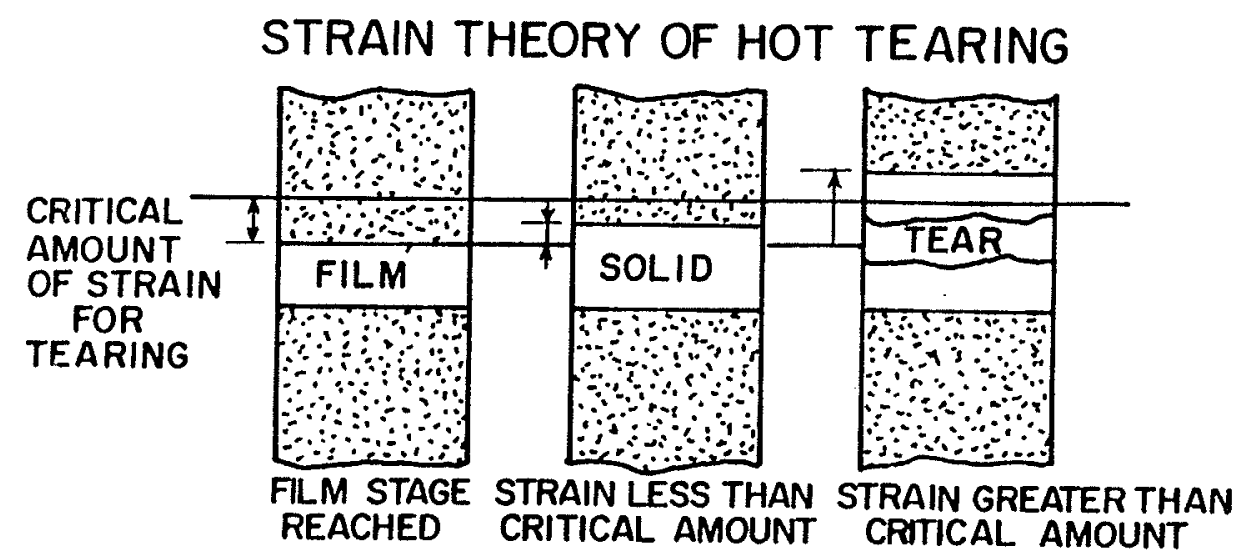

TOTAL STRA IN DEVELOPED DURING FILM LIFE PERIOD DEPENDS ON:

(1) STRAIN RATE

(2) TIME OF FILM LIFE

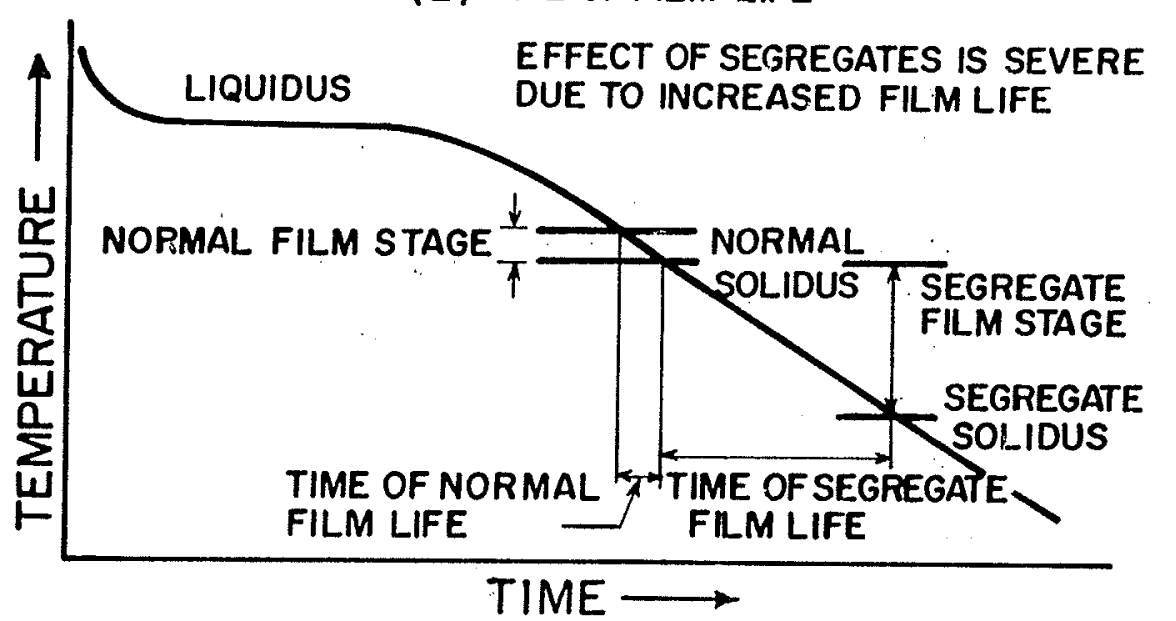

Fig. 2.11 : Illustrating basic concepts of the process by which the total amount and rate of strain (extension imposed on the hot spot zone) concentrated to the liquid films determines the develpment of hot tearings in castings [20]. 
with lower time-rates of extension than would cause hot tearing in absence of liquid film forming elements.

The presence of sulfur and phosphorus not only causes hot tearing to occur with milder mechanical conditions but also causes enlargement and aggravation of tears which begin at the normal high temperatures in the presence of severe mechanical conditions. The wider temperature range of hot tearing provided by these elements permits enlargement of initial cracks in true hot tear fashion. Thus, as is normally observed, liquid film forming alloying elements produce more numerous and more severe hot tears.

Radiographic studies by Pellini [20] on experimental aluminum and steel castings with varying carbon contents during solidification showed that in all cases the first tear occurred above the solidus temperature. Radiographic studies of experimental castings made from tin-bismuth alloys containing upto $58 \%$ bismuth, with step wise cooling and holding in mold at various temperatures, have also shown that hot tears are formed at temperatures slightly above the solidus [28]. Saveiko [29] has put forward ideas which are in part a mathematical model of the strain theory at least in terms of the liquid rupture event. Furthermore, Saveiko [29] was the first to recognize the role of surface tension and thickness of the intergranular liquid film, the two important factors that were not recognized by Pellini. According to Saveiko [29], the surface tension and the thickness of the intergranular liquid film are the controlling factors in the cracking process. Saveiko descibes the hot tearing process on the microscopic scale as follows:

During solidification, when linear shrinkage starts and resistance is offerred to it, the system is subjected to tension, as indicated in fig 2.12 [29]. The forces required to shear the grains relative to one another along vertical faces are small and can be neglected. Thus, the system can be represented as consisting of solid metallic matrix separated by liquid films, fig 2.12, lower right. As shrinkage proceeds, each microscopic volume in the massive section is subjected to extension; being liquid, the films extend only very slightly, while the metallic matrix is extended substantially, fig 2.13 (a) [29]. Hot tearing will occur 


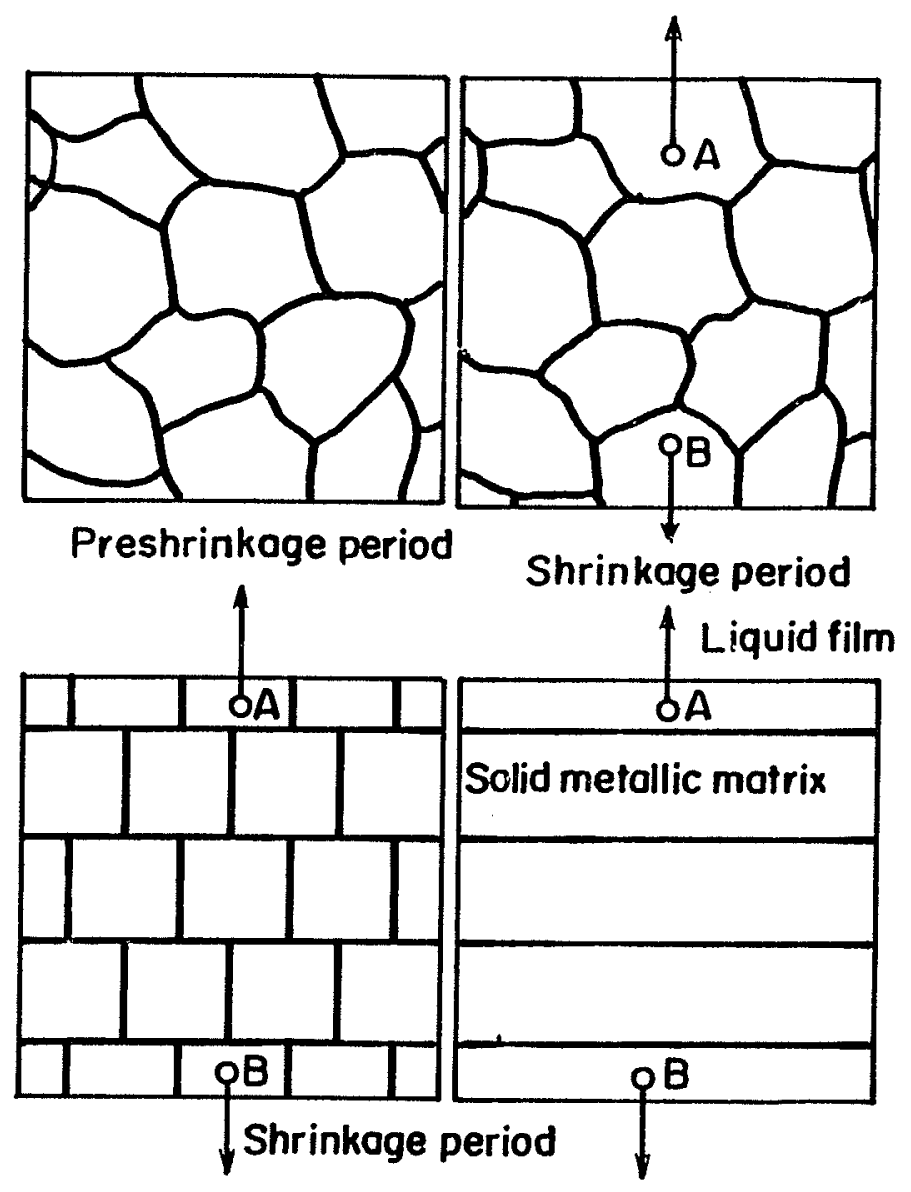

Fig. 2.12: [29] 


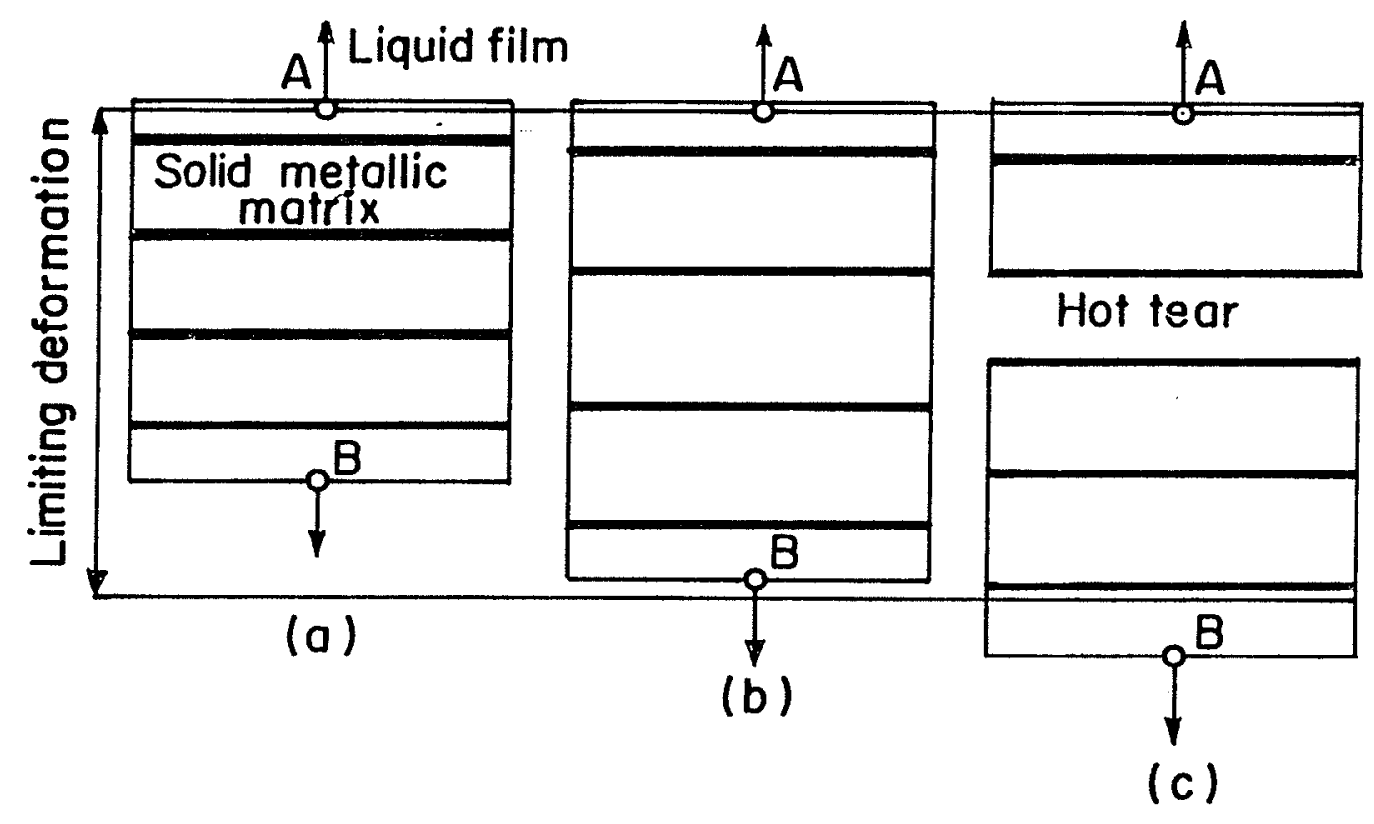

Fig. 2.13: [29] 
only when the absolute deformation in the massive section has reached a certain definite value. This implies [fig 2.13(c)], that the total deformation in length $A B$ has reached its limiting value and point $B$ has moved further than it would correspond to limiting deformation, by forming a tear along one of the liquid films. If the extension of length $A B$ does not exceed the limiting deformation, hot tearing will not occur, fig 2.13(c).

As the alloy cools the absolute value of shrinkage increases. Accordingly, if shrinkage is prevented, the hot tearing zone is increasingly extended. This extension takes place in the solid metallic matrix i.e., in the grains, so that the stress level within the grains increases. At the same time the stresses in the liquid films increase. Eventually the stresses reach the same level as the specific value (per unit area) of the forces due to surface tension in the intergranular film and to its thickness; the film is torn and a hot tear forms.

The force applied at right angles required to tear apart the liquid film is [29]:

$$
P=\frac{2 \alpha F}{1000 g b} k g
$$

where $\alpha$ is the surface tension of the liquid $\left(\mathrm{ergs} / \mathrm{cm}^{2}\right.$ ); F is the cross sectional area of hot tearing zone $\left(\mathrm{cm}^{2}\right)$; $b$ is the thickness of the liquid film $(\mathrm{cm})$; and $\mathrm{g}$ is the acceleration due to gravity $\left(\mathrm{cm}^{2} / \mathrm{s}\right)$. Hence, it can be seen that the lower is the surface tension of the liquid film, the lower the resistance to hot tearing.

The composition and nature of the liquid film are functions of the alloy composition and the casting cooling conditions. Accordingly, the film volume is constant under given conditions. The increased total surface area of the grains in a fine grained casting decreases the volume of film per unit surface area and thus its thickness. Since the tearing force is inversely proportional to the film thickness, the hot tear resistance increases.

\subsubsection{1(c): Generalized theory of super-solidus cracking ${ }^{[23-25]}$ :}

This theory proposed by Borland [23-25] emphasizes three effects, viz., the effect of freezing mode, the effect of interphase and grain boundary energies and the effect of solute additions as primary factors in determining the hot cracking susceptibility. 


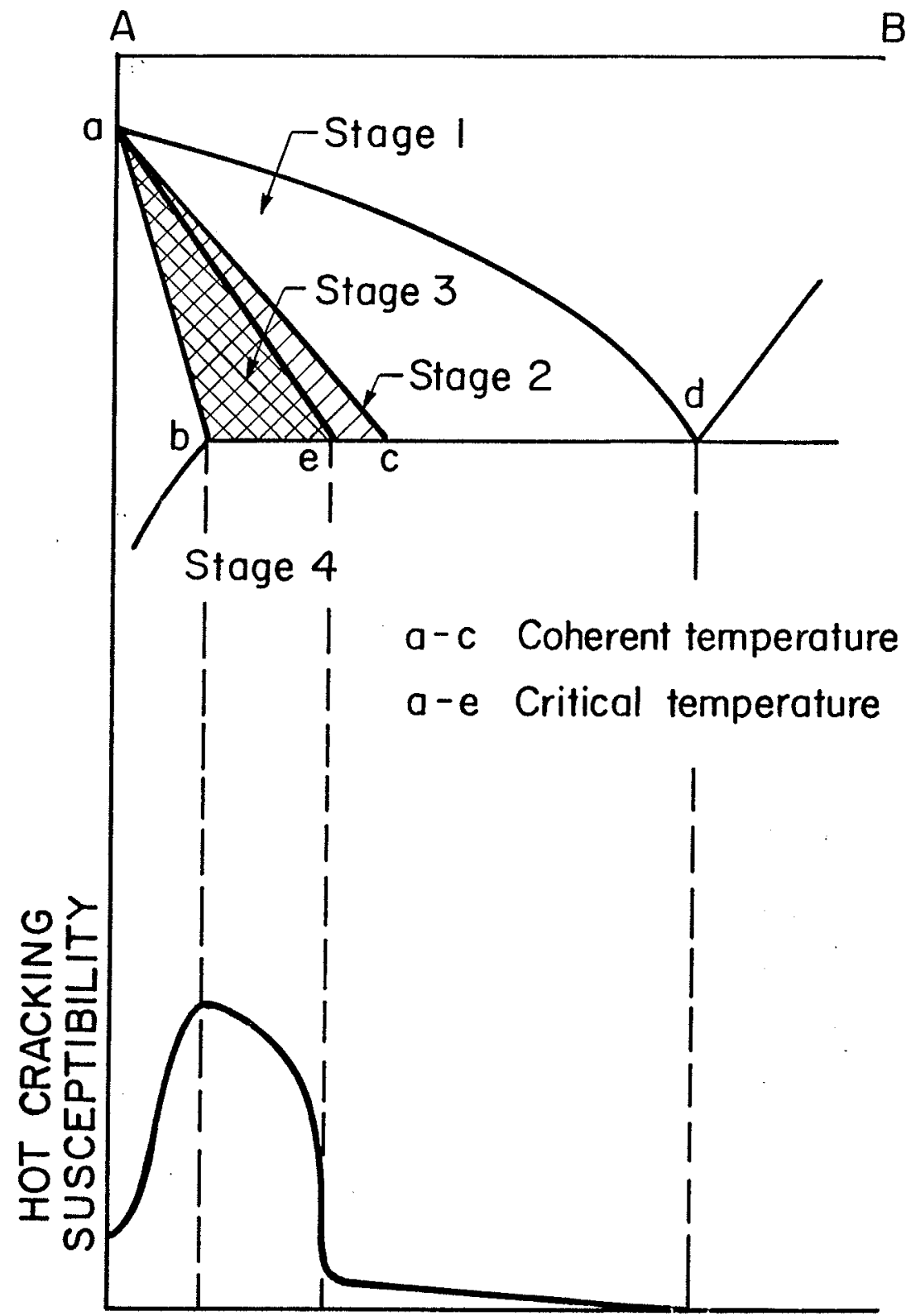

Stage 1 -Dendrites freely dispersed in liquid. No cracking. Stage 2-Interlocking of grains.' Liquid healing' possible if cracks form. 'Accommodation' not important

Stage 3-Critical solidification range. No 'healing' of cracks possible if 'accommodation' strain exceeded

Stage 4 - Solidification. No crocking a-c-e-Brittle ronge

Fig. 2.14: Effect of constitutional feotures on cracking susceptibility in binary systems [23]. 
Effect of freezing mode: Following Portevin and Dannemuller [30] the freezing process may be divided into four stages, fig 2.14 [23]. These stages are:

Stage 1: Primary dendrite formation: where the solid phase is dispersed in a continuous liquid and both solid and liquid phases are capable of relative movement.

Stage 2: Dendritic interlocking: where both liquid and solid phases are continuous, but only the liquid is capable of relative movement and is able to circulate freely between interlocking dendrites.

Stage 3: Grain boundary development: where the solid crystals are in an advanced stage of development and the semicontinuous network restricts the free passage of liquid. The relative movement of the two phases is impossible during this stage.

Stage 4: solidification: where the remaining liquid is solidified.

Healing may be possible only in stage 2 if sufficient liquid is present, since beyond this stage the crystal formation prevents the free passage of liquid. Accommodation is possible once the dendrites join together, but it really becomes important only in stage 3 where cracks formed cannot be healed.

Effect of interface and grain boundary energies: For cracking to occur it is not a sufficient condition that a wide freezing range should exist; not only should the alloy pass through a liquid stage but the liquid should also be present over a relatively wide temperature interval in a form that will allow high stresses to be built up between grains. $A$ liquid phase covering all most all the grain faces during freezing will allow the development of high stresses on the narrow bridges joining adjacent grains, but liquid confined to grain edges and grain corners will prevent these high stresses being built up.

The distribution of liquid during cooling is related to interfacial energies of the liquid and solid concerned. However, under welding conditions, near equilibrium values would be established only at very high temperatures. Following Smith [31], the interface and grain boundary energy can be related to the dihedral angle as

$$
\frac{\gamma_{S L}}{\gamma_{S S}}=\frac{1}{2 \cos (\theta / 2)}
$$


where $\gamma_{S L}$ is the interphase (solid-liquid) energy, $\gamma_{S S}$ is the grain boundary energy and $\theta$ is the dihedral angle.

The ratio $\tau=\gamma_{S L} / \gamma_{S S}$ is of particular importance. The dihedral angle is zero for $\tau=$ 0.5 and all values below. The relation between dihedral angle and $\tau$ for $\tau \geq 0.5$ is shown in fig 2.15 [23].

It has been suggested that, in alloy systems where extensive solid solutions occur, when the liquid is substantially similar in composition to that of the previously frozen solid, 'wetting' of the grain faces and the edges will almost be complete. If this condition exists in the weld pool over a relatively large temperature interval while it is freezing then it can be reasoned that cracks could form under adverse strain conditions, because only small areas of the grain faces will then be joined. On the other hand, when metals are immiscible in the molten state and one metal solidifies $\tau$ will be much greater than 0.5 , and the liquid will be largely confined to the grain edges and corners. In these systems the cracking susceptibility will be low.

In general as $\tau$ increases above $0.5(\theta=0)$ the liquid progressively occupies less of the grain faces, and at $\tau=0.57\left(\theta=60^{\circ}\right)$ it can exist only as a continuous network along the grain edges. For values of $\tau \geq 0.57$ the liquid phase will collect at the grain corners and will extend progressively less along the grain boundary edges as $\tau$ increases. (fig 2.16). When $\tau$ is less than 0.5 , however, the liquid is able to penetrate between existing grain boundaries, and in the process destroys the cohesion between adjoining grains. In this condition, however, the stresses imposed upon the crystals during freezing should be virtually nil.

During freezing $\tau$ increases owing to the change in difference between solid and liquid compositions (assuming no phase change takes place) and for most alloys reaches a value greater than 0.5 before solidification is complete. It was suggested by Borland that $\tau$ governs the distribution of liquid during the final stages of solidification (i.e below the commencement of stage 3 of solidification) when grain boundaries are being developed. 


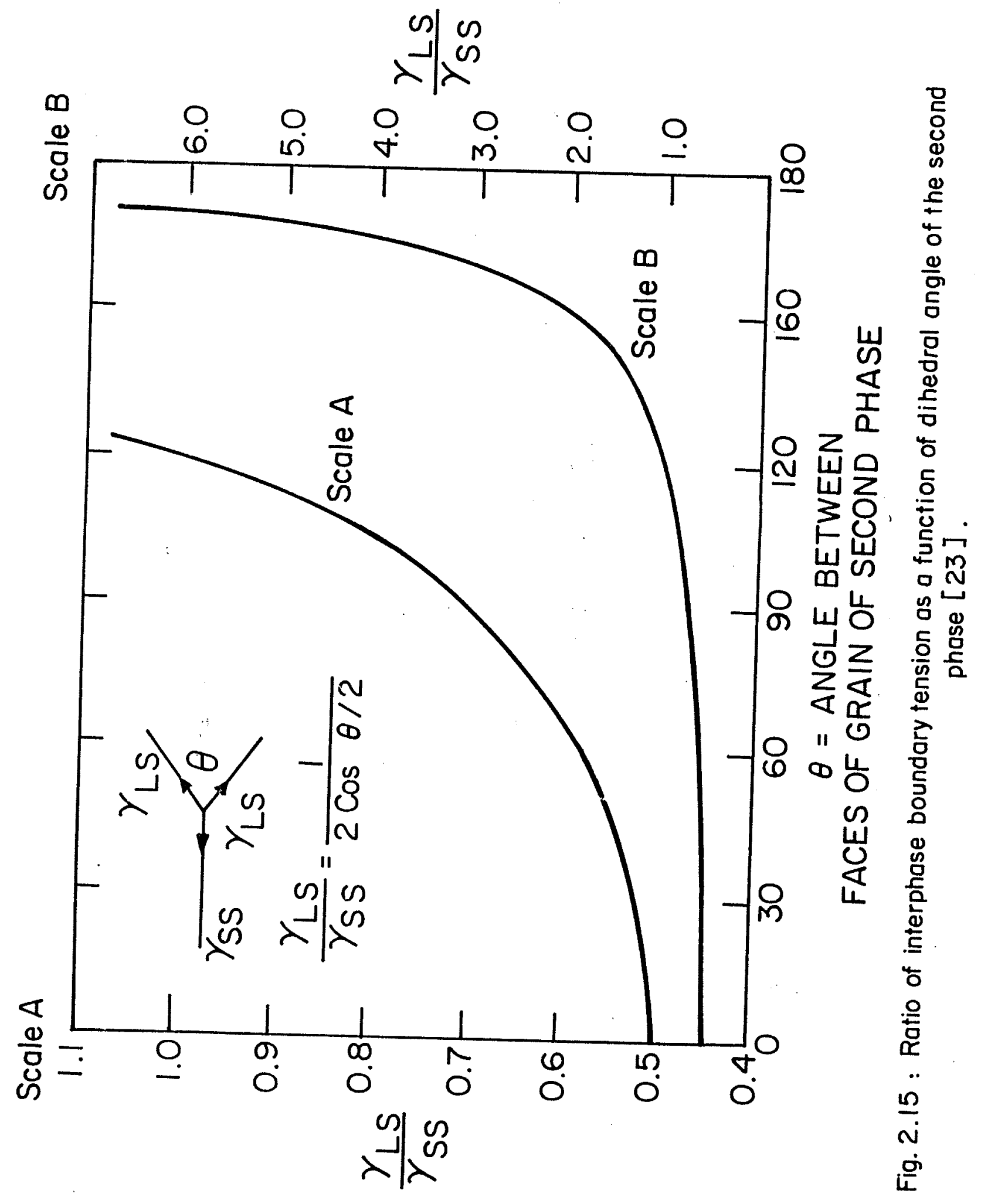



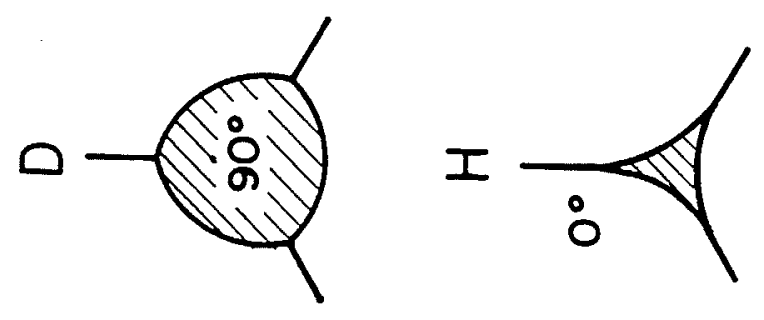

$-\mid \begin{aligned} & N \\ & D \\ & \delta \\ & \delta \\ & N\end{aligned}$
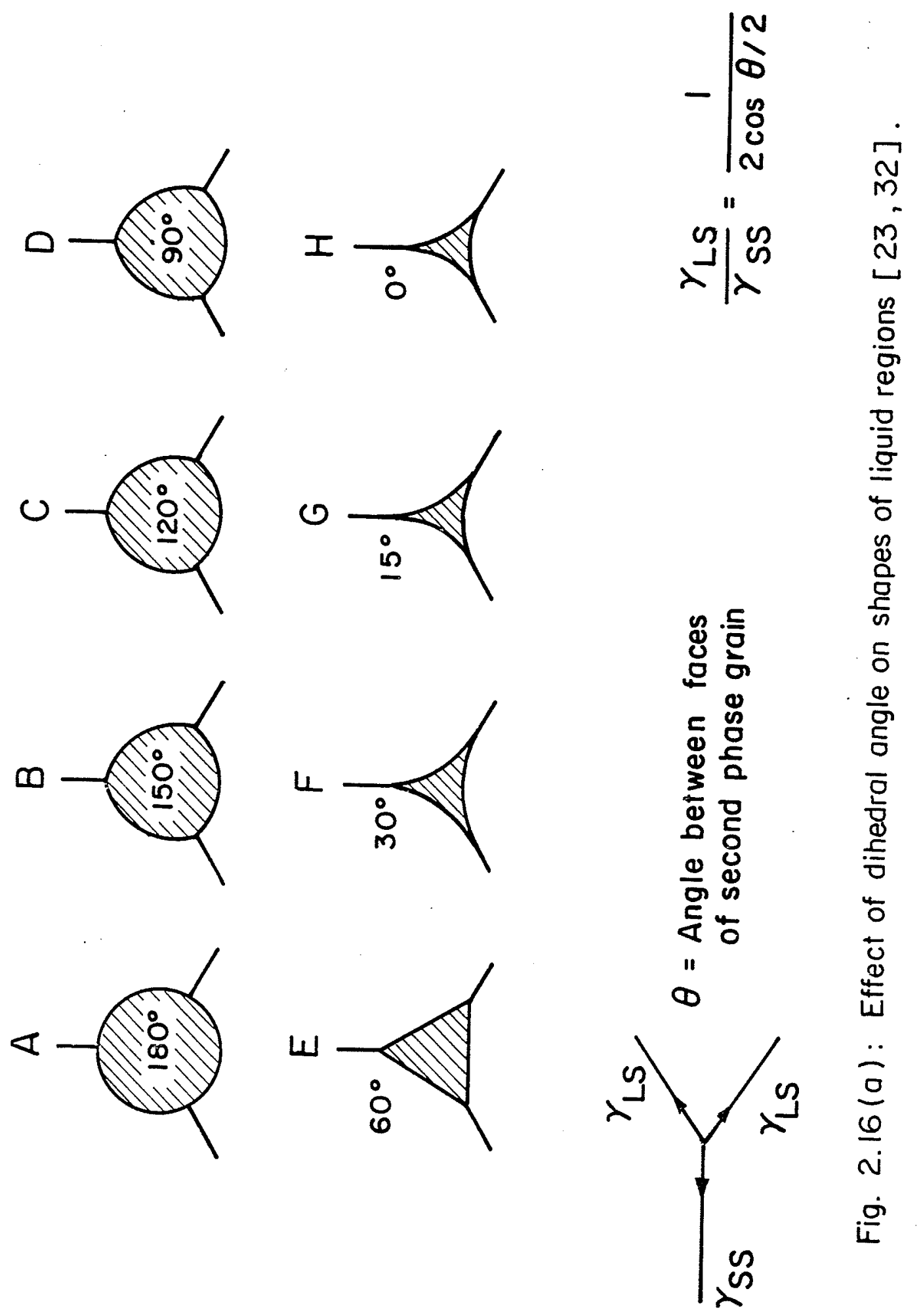

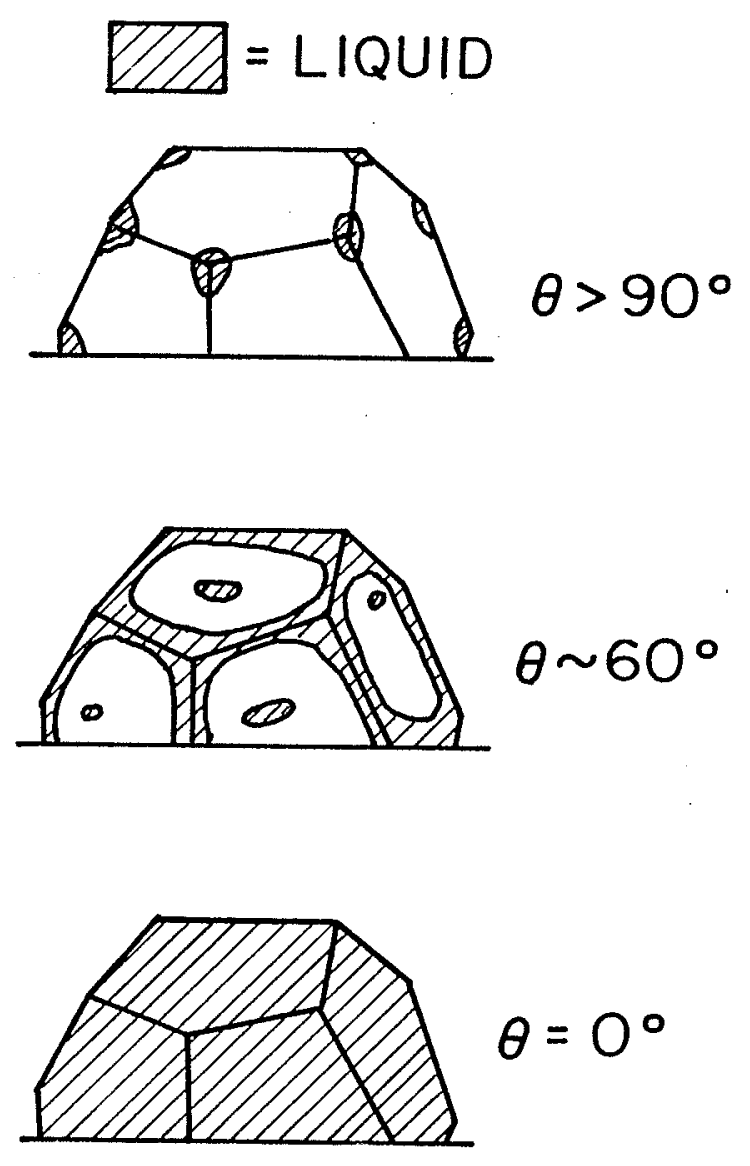

Fig. 2.16 (b): Effect of dihedral angle on distribution of liquid phases on grain corners edges and faces $[23,32]$. 
A high value of $\tau$, say 0.57 , manipulates the liquid so as to allow the grain faces to join and so provide a strong bond and thus prevent the formation of microfissures. On the other hand, a value of $\tau$ near 0.5 allows the liquid to remain at grain faces as an almost continuous film and prevents them from joining quickly; this allows high stresses to be built up locally and fissures to be developed. As a corallory, it may be argued that if continuous liquid films exist then fissures are unlikely to be formed because of the small stresses imposed upon the separating liquid films. Bridging between grains cannot occur, nor can a C.S.R. (critical solidification range, i.e, stage 3 of solidification, fig 2.14 be established. Ideally, therefore, fissures will not form when $\tau$ is less than 0.5 . In practice this condition is only satisfied when a pure metal solidifies. Solid solutions should also freeze in the manner as pure metals but, because of the relatively fast cooling rates, equilibrium conditions will not be obtained and $\tau$ values of just greater than 0.5 are developed. Hence, under non-equilibrium freezing conditions, solid solutions are inherently susceptibile to cracking.

Borland generalizes the above factors and proposes that the increasing value of $\tau$ with cooling below the critical temperature competes with the increasing thermal strains, and that fissuring is prevented only when the stresses imposed on the bridges between growing dendrites does not exceed a critical amount. This stress is a function of the grain boundary area that is joined and not seperated by the liquid phase. The distribution and quantity of the liquid is influenced by the grain size and shape, and the effect of temperature and cooling rate on the slope of the liquidus and solidus curves. These slopes in turn determine the composition of the liquid that largely determines the value of $\tau$.

Under welding conditions it seems unlikely that the effective value of $\tau$ in the C.S.R. is developed during the first few moments of freezing. At lower temperatures equilibrium values would not be established and thus the $\tau$ values created at the higher temperatures will persist. For alloys containing small amounts of solute, it would seem that the initial slope of liquidus is very important. Borland also suggests that the extent of C.S.R. below 
the crack initiation temperature assumes a more important role in crack propogation than the dihedral angle.

Effects of solute additions: The form of the diagram (fig 2.14) showing the cracking tendancy in a binary system is due to the following circumstances: With increasing amounts of solute, $T_{C}$ will be depressed because of the greater amounts of liquid persisting to lower temperatures; at the same time the solidus is lowered due to changes in solid solubility. As the extent of the freezing range thus increases so does the C.S.R. because of the greater amounts of non-equilibrium solidification. The net effect is to increase the cracking tendancy. This will continue until the solidus is not depressed further (limit of solid solubility) and if at the same time $T_{C}$ is lowered further, cracking will decrease due to the consequent shortening of the C.S.R. Below the solid solubility limit the $\tau$ value will probably remain at just above 0.5 but will increase more rapidly as this limit is exceeded and a eutectic composition is approached. It has been emphasized, however, by Borland that the establishment of C.S.R. is due to non-equilibrium freezing and the creation of $\tau$ values greater than 0.5 .

Hence according to the theory developed by Borland, an alloy is highly susceptible to cracking when the composition of the liquid in contact with the growing crystals changes very little over relatively wide temperature interval. Binary systems, that to a large extent satisfy these conditions, are those which exhibit primary solid solution range with a near vertical solidus, and a liquidus which, initially, does not change too rapidly with a fall in temperature. Alloys most susceptible to cracking should be those which contain small amounts of the solute element. Alloys which contain a large amounts of solute have a liquid composition somewhat different from that of the solid. An important implication of the generalized theory is that cracking may be prevented in a crack susceptible alloy if the composition of the liquid is modified so that it is significantly different from that of the solid.

Rogerson and Borland [32] have shown that the sizes of the dihedral angles of the 
solid-liquid interfaces at super-solidus temperatures can be correlated with the degree of embrittlement above the solidus as shown by the tendancy towards hot cracking during solidification of welds and castings. They suggest that one of the metallurgical factors that determine the hot cracking susceptibility is the type and distribution of the intergranular liquid during solidification. It was shown by these authors that welds in $\mathrm{Al}-\mathrm{Sn}$ alloys which contained extensive grain boundary liquid films cracked extensively while $\mathrm{Al}-\mathrm{Cd}$ and $\mathrm{Al}-\mathrm{In}$ alloys that contain globular liquid films did not crack at all. In a later article, Borland proposes that cracking could be due to liquid metal embrittlement based on the general belief that the presence of liquid phases at the grain boundaries of solid metal causes embrittlement because of the lack of sufficient cohesion between the grains. Borland was one of the first to recognize crack initiation and crack propagation as two distinct events, each being aided by a different metallurgical factor. He was also the first to appreciate that crack initiation could occur (i) by parting across the solid-liquid interface (ii) rupture within the liquid film or (iii) by necking and parting of the liquid film. Following Borland [25,33], these aspects are described below:

Borland assumes that the association of cracking and the presence of "essentially continuous films" is proven despite the kind of operating fracture mechanism. He suggests that the liquid coverage of partially covered grain faces can be increased by liquid penetration aided by an imposed stress and that cracking can occur in the same way as when almost continuous or continuous liquid films are present. When cracks do initiate for whatever reason, they are able to spread easily along the liquid path by a necking of the liquid film. The liquid penetration mechanism could also increase the extent of crack propagation further so long as sufficient stress levels are present and the liquid can continue to reach the crack tip. Cracking at the tip could of course possibly happen in the absence of the liquid but then this would be a solid-solid tearing process. To incur solidification cracking, therefore, the liquid films should be extensive in their coverage of grain/interdendritic surfaces or there must be a means of extending a sub-critical coverage. 
In the fracture of solids aided by a liquid metal environment there are at least three important aspects to consider: (i) The presence and/or penetration of liquid between grains or along crystallographic planes, (ii) fracture initiation at solid-liquid interface or parting within the liquid phase, and (iii) propogation of the crack.

Consider first the energies of formation involved in the seperation of solid and liquid components. The liquid zone extends right across the cross-section of the specimen, so that the liquid is open to the surface. For the separation of the solid-liquid surface and within the liquid phase, the energies involved are, respectively, $\gamma_{L V}+\gamma_{S V}-\gamma_{S L}$ and $2 \gamma_{L V}$ where $\gamma_{L V}$ is the liquid-vapor interfacial energy, $\gamma_{S V}$ is the solid-vapor interfacial energy, and $\gamma_{S L}$ is the solid liquid interfacial energy (fig 2.17 [25]).

For a hypothetical case of pure metal in contact with its own melt, the energies of formation are identical. In the practical sense, however, parting within the liquid assuredly occurs as the liquid at the solid-liquid interface wets the solid and remains in contact with it and the liquid 'necks' down and finally separates a little distance from the interface (fig 2.17). The shear stresses that liquid can support are extremely small. The strain necessary to cause necking of the liquid film will depend on the film thickness. The thicker the film the greater the strain required. For separation to occur at the solid-liquid interface with this type of specimen, the liquid would have to be practically non-wetting.

The force required to separate a liquid film not exposed to the surface increases as the film thickness decreases according to the expression (same as the one used by Saveiko [29])

$$
F=\frac{2 A \gamma_{L V}}{d}
$$

where $A$ is the area of the liquid film attached to solid and $d$ is the thickness of the film. When the liquid phase is contained internally and not open to surface, the various possibilities are: (i) separation at the solid liquid interface $\gamma_{L V}+\gamma_{S V}-\gamma_{S L}$ (ii) separation within the liquid $2 \gamma_{L V}$ and (iii) penetration of liquid into the solid (e.g. along grain boundaries) $2 \gamma_{S L}-\gamma_{b}$. 

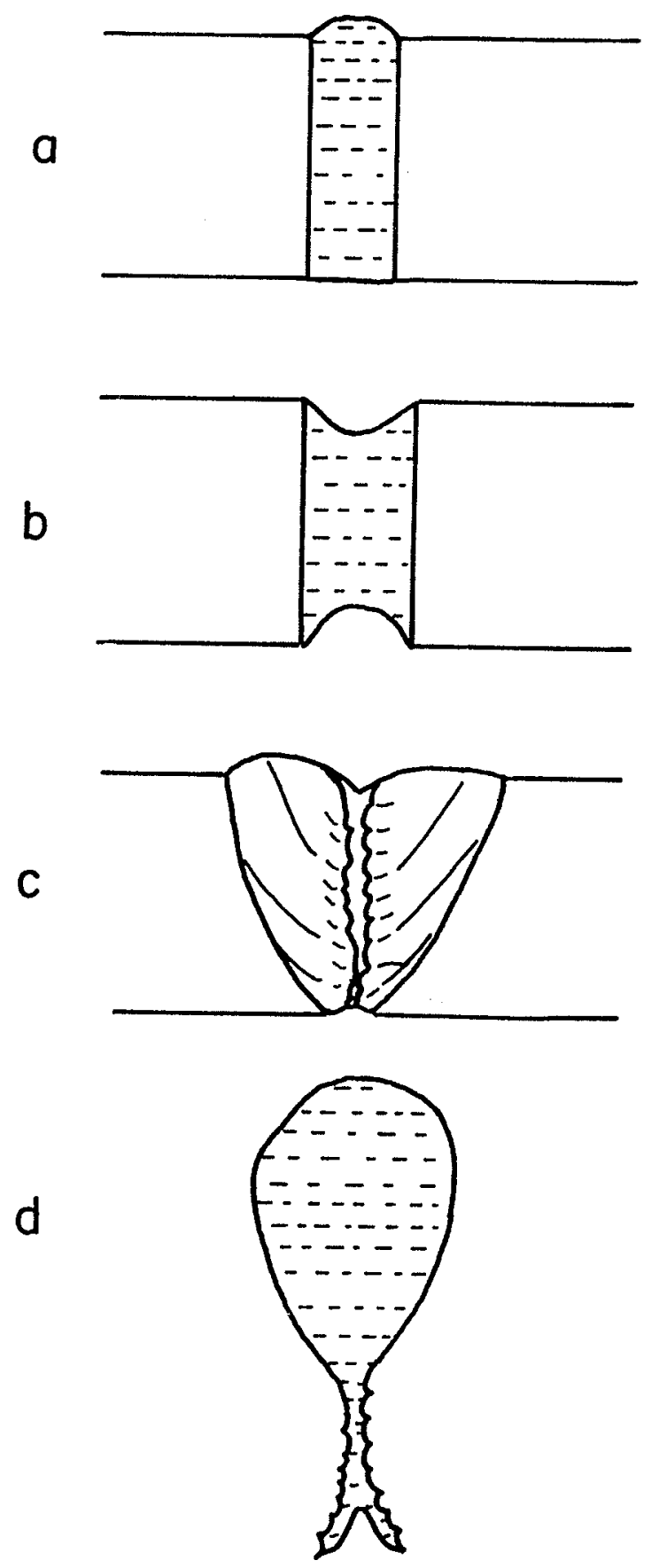

Fig. 2.17: Fracture of solid-liquid components in a weld (thin section) (a) Fracture in liquid $\left(2 \gamma_{L V}\right)$ and fracture at interface $\left(\gamma_{L V}-\gamma_{S V}-\gamma_{S L}\right)$; necking of liquid at free surface due to application of very low strains (c) and (d) liquid film and dendrite formation ot the rear end of weld pool during fracture process [25]. 
For the liquid to separate without first necking down (since it is constrained by the solid walls) the required fracture stress would be exceedingly high and only a little less than theoretical strength of the solid.

$$
\sigma_{\text {liquid }}=\sigma_{\text {Solid }}
$$

The remaining possibilities are that fracture initiates at the solid-liquid interface of that grain boundary where penetration by liquid occurs. Separation at the solid-liquid interface is likely when

$$
\gamma_{L V}+\gamma_{S V}-\gamma_{S L} \leq 2 \gamma_{S L}-\gamma_{b}
$$

Assuming that $\gamma_{S V}=3 \gamma_{b}$ and substituting $\gamma_{b}[\sec (\theta / 2)-1]$ for $2 \gamma_{S L}-\gamma_{b}$ the inequality then becomes

$$
\gamma_{b}[\sec (\theta / 2)-1] \geq \gamma_{L V}-\left(\gamma_{b} / 2\right)[\sec (\theta / 2)-6]
$$

Taking $\gamma_{L V}$ to be zero as a mathematical convenience (the lowest possible value), then for the rupture to occur at the solid-liquid interface, $\theta=136^{\circ}$. For the greater values of $\gamma_{L V}$, $\theta$ is corresponding higher. It is thus seen that for low dihedral angles, liquid penetration is the more likely event. From this, Borland tentatively concluded that while continuous liquid phase provides an easy extension path for an existing crack, the presence of the liquid does not necessarily account for the formation of the crack. Cracks are more likely to initiate at the interface of liquid particles with very high dihedral angles. A larger number of particles with very high dihedral angles, according to Borland, could possibly be more damaging than a more continuous phase of low dihedral angle. The stress for liquid metal penetration is

$$
\sigma_{a}=\left[\frac{E \gamma_{b}\{\sec (\theta / 2)-1\}}{4 C}\right]^{1 / 2}
$$

where $E$ is the Young's modulus and $C$ is the crack length (liquid filled or liquid at crack tip).

$$
\gamma_{b}[\sec (\theta / 2)-1]=\left[2 \gamma_{S L}-\gamma_{b}\right]
$$


It should be noted that $\sigma_{a} \longrightarrow$ zero as $\theta \longrightarrow$ zero. Also, $C$ is twice the equivalent grain diameter concept since the middle point of the liquid film resides at the boundary between two grains.

In AI-Sn alloys, Borland has studied the effect of temperature on the ductility (elongation) and strength by subjecting tensile specimens to simulated weld thermal cycles and testing at various temperatures during solidification. The coherent temperature was determined from the data as the temperature at which the alloy starts gaining strength. His ductility data prove the existence of a ductility dip during solidification. The range of this ductility dip then defines the B.T.R for the system. The important aspects of the observed ductility trough are (i) the temperature at which the ductility first rapidly decreases (ii) the minimum ductility (iii) temperature or temperature range at which the maximum ductility is reached and (iv) temperature at which the ductility begins to increase rapidly. Borland's results show that the coherent temperature is reached before the ductility begins to increase. This indicates that some solid-solid bridging has occurred but not sufficiently extensively to cause a marked raise in ductility. The temperature difference between the initial decrease in ductility and the coherent temperature is a measure of the temperature range in which there is a liquid phase present to separate the grains and prevent solid-solid bridging.

Hence, Borland's results on Al-Sn alloys indicate that there is a brittle temperature range during solidification where cracks could be initiated by the application of a small strain. If the critical strain is applied above the coherent temperature then the crack will be due to the parting/necking of the liquid seperating the solid phases. Strains applied below the coherent temperature involve the breaking of the solid-solid bridges. Borland, however, does not specify whether or not the critical separation strains for specific welding conditions are achieved in the liquid film zone or in the zone where solid-solid bridging has commenced. 


\section{Subsolidus Cracking}

The essential requirements for subsolidus cracking are the same as for supersolidus cracking: viz. brittleness combined with tensile stress. A pre-existing crack and/or a stress-raising configuration will further increase the risk of failure. Subsolidus cracking may occur in the weld zone or in the HAZ. Embrittlement is an important contributory factor to subsolidus cracking. Embrittlement mechanisms are usually time dependent so that cracking may occur at any time after solidification. Some times cracks will occur after months of storage or use or during some post welding operation such as heat treatment. The causes of subsolidus cracking are often complex and difficult to elucidate, and in some cases it is difficult to determine with certainty whether the cracking is of the supersolidus or subsolidus type. A number of more crack sensitive alloys are prone to both these types of failure, and it is not uncommon for a subsolidus crack to be triggered by a pre-existing crack that formed during welding.

\subsubsection{2: Review of Heat Affected Zone Liquation Cracking Theories:}

The term 'HAZ liquation cracking' is used to indicate the cracking that occurs in the heat affected zone of a weldment. The bulk of published literature devoted to HAZ liquation cracking has focussed on the behavior of austenitic stainless steels and nickelbase superalloys. Austenitic stainless steels which have been reported to be susceptible to HAZ liquation cracking include stabilized grades containing titanium or niobium such as type 321 [34,35] and type 347 [34-36] and high alloyed materials such as Incoloy Alloy 800 [37-39] and A-286 [39,40]. HAZ liquation cracking has been reported in both cast and wrought versions of a wide variety of nickel-base superalloys including alloy $718[16,41,42]$, Hastelloy $X$ [43] and Udimet 700 [1]. These alloys have provided the basis for many of the HAZ liquation cracking mechanisms that have been proposed.

Mechanisms used to describe HAZ liquation cracking can be divided into two general categories; those that support a grain boundary penetration mechanism and those in 
which grain boundary segregation is important. The metallurgical basis for the penetration mechanism is the phenomenon of 'Constitutional Liquation' first proposed purely on theoretical grounds by Savage and popularized by Savage and co-workers [44-46]. Constitutional liquation is a non-equilibrium phenomenon associated with the rapid heating rates experienced in the $H A Z$ adjacent to the fusion line. As certain constituent particles are rapidly heated to temperatures below the matrix solidus temperature, localized liquation occurs along the particle-matrix interface. This liquation is a consequence of the rapid dissolution of the constituent particle at elevated temperatures and the resulting reaction at the particle-matrix interface. The amount of liquid that forms along the interface depends on the heating rate, the dissolution kinetics of the constituent particle, and the diffusivity of solute atoms in the matrix. For example, if heating rates are extremely rapid, as in the $\mathrm{HAZ}$ of an electron beam or a laser weld, particle dissolution would be negligible and constitutional liquation would be minimized. Conversely, if the heating rates in the HAZ are slow, as in electroslag weld, particle dissolution approaches equilibrium conditions. Solid state particle dissolution thus precludes the onset of constitutional liquation. In practice, constitutional liquation is mostly observed when susceptible materials are welded using the more conventional welded processes such as submerged metal arc welding (SMAW), gas tungsten arc welding (GTAW), gas metal arc welding (GMAW), etc. The following section decribes in detail, following Pepe and Savage [46], the phenomenon of constitutional liquation.

\subsubsection{1: Constitutional liquation ${ }^{[46]}$ :}

Assume a hypothetical binary alloy system exhibiting the behavior summarized by the portion of the phase diagram shown in fig 2.18 [46]. Consider an alloy of nominal composition $C_{0}$ which at temperature $T_{1}$ consists of the high melting point phase $\beta$ in the matrix of $\alpha$ solid solution. With an infinitely slow rate of heating, the two phase alloy is gradually converted to a single phase alloy and so remains until the first melting 


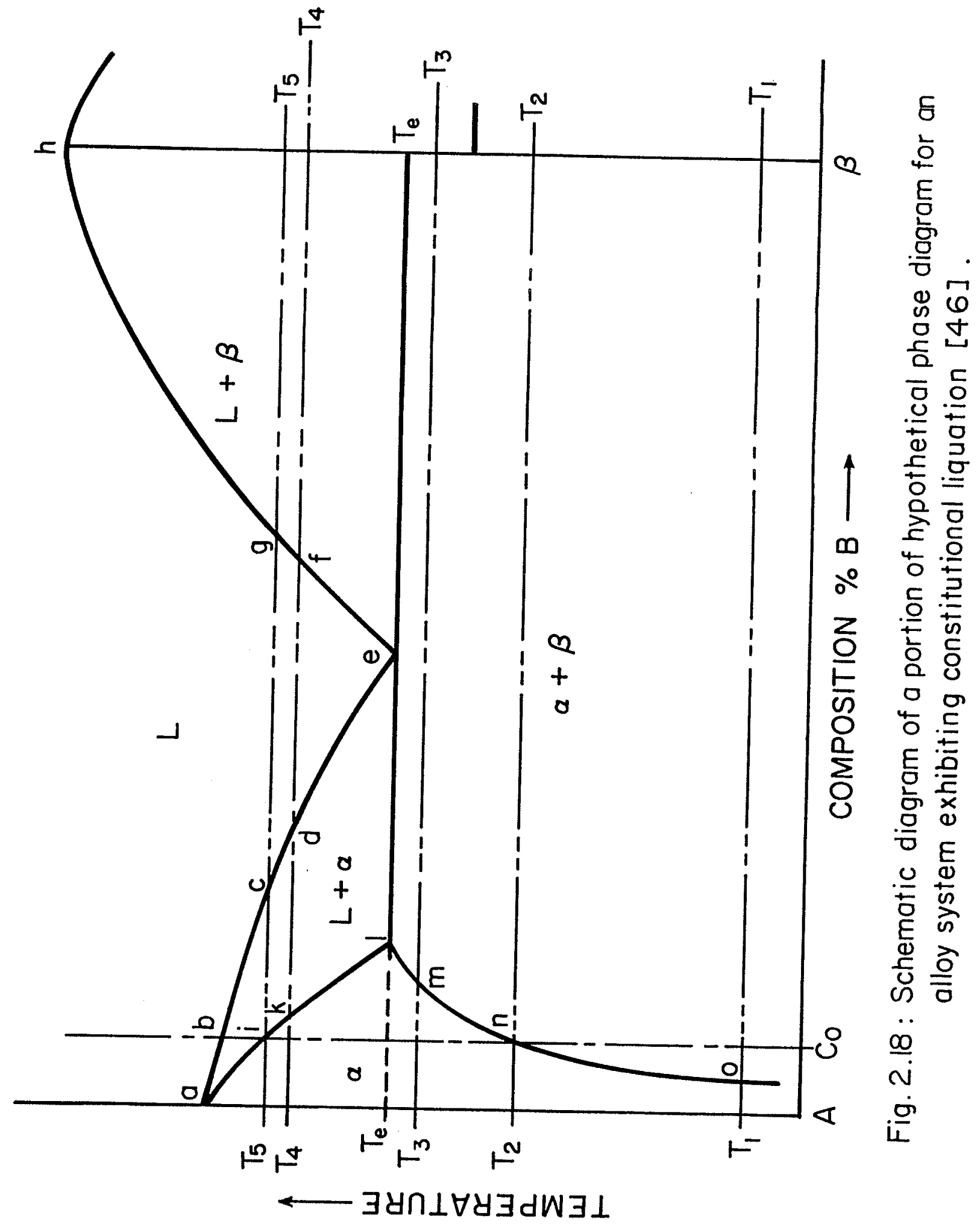


occurs upon reaching the equilibrium solidus. If the alloy is heated from $T_{1}$ to $T_{4}$ at a rapid rate corresponding to nonequilibrium behavior, $\beta$ can only dissolve partially, since the dissolution of $\beta$ is diffusion controlled. In further discussion, it is assumed that the heating rates are rapid at all times and the temperature and compositions cited will be defined in terms of the labels provided in fig 2.18 .

Assuming that $\beta$ phase is spherical, figs 2.19(a) through fig 2.19(c) [46] show schematically the changes expected in the vicinity of the precipitate during heating to temperatures of $T_{3}, T_{c}$ and $T_{4}$ respectively. The equilibrium microstructure for an alloy of this composition as a function of temperature is represented by the vertical line emanating from the composition $C_{0}$ in fig 2.18. Any departures from an equilibrium structure due to rapid heating result in redistribution of solute since the system will be striving to maintain equilibrium.

During heating to temperature $T_{3}, \beta$ begins to dissolve as at $T_{3}, \beta$ is unstable with respect to a single phase solid solution of composition $C_{0}$. Thus, the phase $\beta$ should shrink somewhat from its original size (represented by the dashed circle in fig 2.19 (a) to a smaller size (represented by the solid circle in fig 2.19(a)). The solute atoms thus liberated should diffuse into the adjacent $\alpha$ matrix. However, fig 2.18 shows that the $\beta$ phase must be in contact with the $\alpha$ phase of composition ' $m$ ' if the two phases are to coexist. Thus, it is necessary that the concentration gradient in the $\alpha$ phase have a maximum concentration of ' $m$ ' at the $\beta$ phase interface and decrease toward the original matrix composition (represented by point 0 ) at the interior of the $\alpha$ phase. The area of the double cross-hatched region of fig $2.19(a)$ must be equal to the single cross-hatched region if a material balance is to be maintained. This will also be true for figs $2.19(\mathrm{a}-\mathrm{c})$ and $2.20(\mathrm{a}-\mathrm{c})[46]$.

The slope of the concentration gradient produced this way should be dependent upon the following:

1. The heating rate-the faster the heating rate, the steeper should be the concentration 

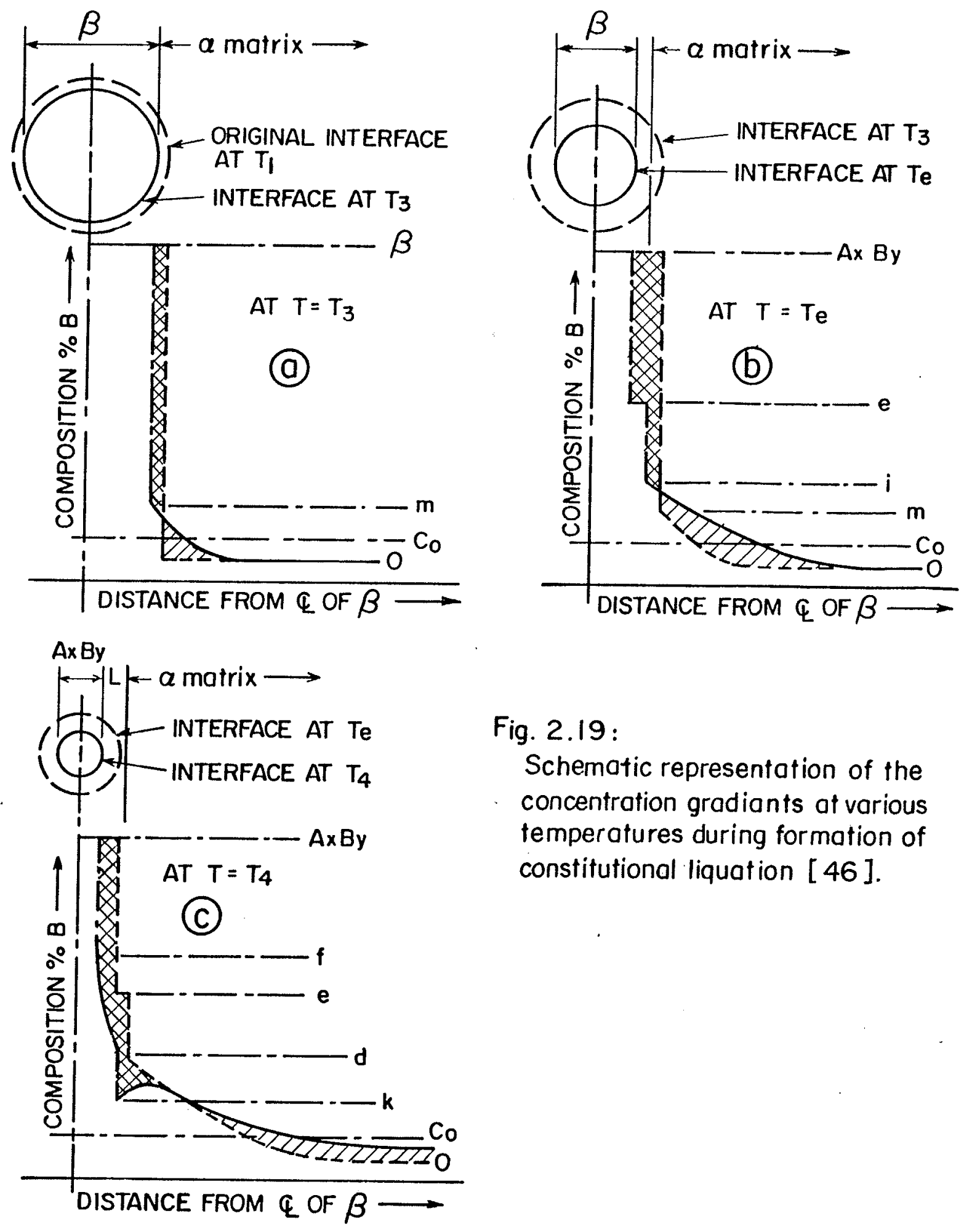

Fig. 2.19:

Schematic representation of the concentration gradiants at various temperafures during formation of constitutional liquation [46]. 


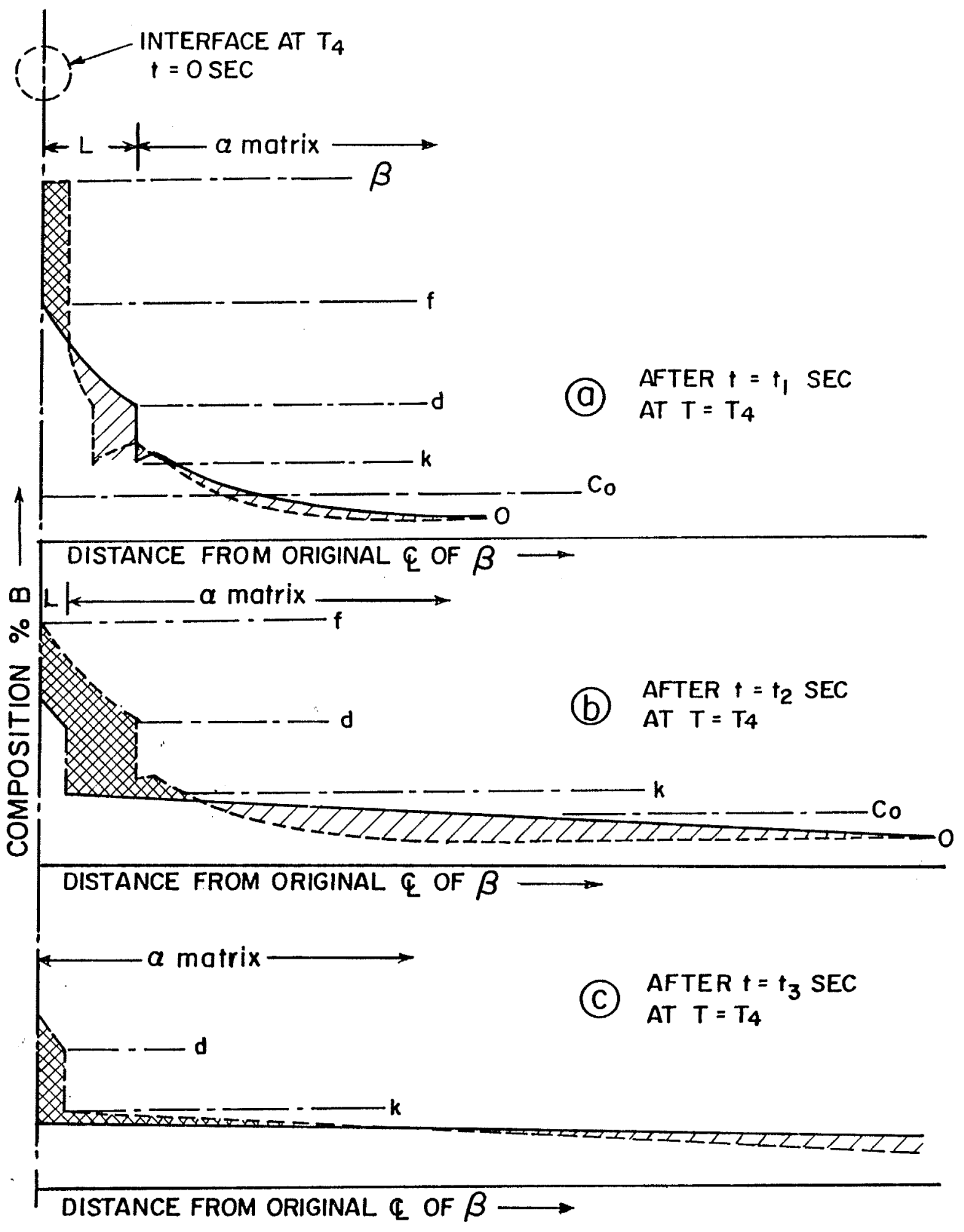

Fig. 2.20 : Schematic representation of the effect of holding a specimen exhibiting constitutional liquation [46]. 
gradient.

2. The diffusivity of the solute atoms-the faster the diffusivity of the solute, the shallower should be the concentration gradient.

3. The relative ease with which the solute atoms are accommodated by each successive single phase region in the diffusion couple.

Fig 2.19(b) shows the additional changes which should occur during heating from a temperature $T_{3}$ to the temperature $T_{e}$, the eutectic temperature. The dashed line of figure represents the solid distribution present at temperature $T_{3}$, and the solid line of fig 2.19(b) represents the modified solute distribution at $\mathrm{T}_{c}$.

During the time interval necessary to heat the material from a temperature $T_{3}$ to a temperature $T_{e}, \beta$ continues to dissolve. This is shown in fig $2.19(\mathrm{~b})$, where the dashed circle represents the location of the particle interface at $T_{3}$, and the solid circle represents the particle interface when temperature reaches $T_{c}$.

At $T_{c}$, the composition corresponding to point ' $\mathrm{e}$ ' permits formation of a single phase liquid at the interface of the $\beta$ phase. Thus, at the instant the temperature of the material reaches $T_{c}$, the undissolved portion of the $\beta$ particle should be surrounded by a liquid phase of composition ' $\mathrm{e}$ ' which, in turn, should be surrounded by the $\alpha$ matrix. The distribution of solute in the three phases coexisting at temperature $\mathrm{T}_{e}$ is shown in fig 2.19 (b).

Further heating to $T_{4}$ would allow additional time for dissolution of the $\beta$ phase and the expected distribution of solute upon reaching the temperature can be represented by the solid line of fig $2.19(\mathrm{c})$. Note that, upon heating above $\mathrm{T}_{c}$, the equilibrium solubility of $\mathrm{B}$ atoms in the $\alpha$ phase decreases along the solidus line 'akl' (fig 2.18). Therefore, as the temperature rises above $T_{e}$, the concentrations of the solid solution and the liquid film in contact with one another should correspond to ' $k$ ' and ' $d$ ' respectively (fig 2.18). This readjustment in composition would create an inverted region in the concentration gradient at this point in time similar to that shown in fig 2.19(c). However, the excess solute in the $\alpha$ phase in this region should cause the $\alpha$ liquid interface to move epitaxially 
into the alpha phase until the excess solute is consumed by forming liquid of composition ' $d$ '. This results from the fact that a solid metal cannot be superheated above its liquidus temperature by more than an infinitesimal amount.

Note that each $\beta$ phase particle remaining undissolved on reaching $\mathrm{T}_{4}$ under these conditions should be completely surrounded by a liquid film of variable composition ranging from ' $f$ ' at the $\beta$ interface to ' $d$ ' at the interface with the $\alpha$ matrix. Thus, localized melting should be possible with rapid heating rates at temperatures below the equilibrium solidus, $T_{5}$. This phenomenon, which in theory could occur at all temperatures above $T_{c}$ is termed constitutional liquation. Evidence of such liquation should be observable whenever an intermetallic compound and the surrounding matrix phase interact to form a low melting liquid phase, provided that the heating rate is rapid enough to preclude the rapid disappearance of the compound prior to reaching the minimum liquidus temperature.

Fig $2.20(a-c)$ summarizes schematically the changes in solute distribution that occurs in a specimen exhibiting constitutional liquation when heated rapidly to temperature $\mathrm{T}_{4}$ and then held isothermally for varying times. After sufficient time, nearly complete homogenization occurs thereby permitting the liquid phase to dissipate completely. The instant after the liquid phase disappears, the composition gradient of $B$ atoms in $\alpha$ phase would exhibit a maximum corresponding to the composition represented by the point $k$ (the maximum solubility of $B$ atoms in the $\alpha$ phase at $T_{4}$ ) at the original centerline of $\beta$ and decrease towards a composition corresponding to point ' $O$ ' at the interior of the $\alpha$ phase. Thus the liquid films produced by constitutional liquation should experience gradual modification in composition once the $\beta$ phase is completely dissolved and should ultimately disappear completely if subjected to a sufficiently long isothermal hold at any temperature below the equilibrium solidus.

The actual situation which prevails during welding is intermediate between these two extremes. The heating rates range from approximately $250^{\circ} \mathrm{C} / \mathrm{sec}$ for a typical fusion weld to over $60,000^{\circ} \mathrm{C} / \mathrm{sec}$ for an electric resistance spot weld, and the average cooling rates 
range from $5^{\circ} \mathrm{C} / \mathrm{sec}$ to $75^{\circ} \mathrm{C} / \mathrm{sec}$ for a typical fusion weld to perhaps $20,000^{\circ} \mathrm{C} / \mathrm{sec}$ for a spot weld.

With interstitial compounds such as $\mathrm{Fe}_{3} \mathrm{C}$, the ease of dissociation and rapid diffusion of the interstitial solute, carbon, make constitutional liquation highly unlikely. This was verified by Aronson [45] who studied constitutional liquation in plain carbon eutectoid steel and concluded that with a heating rate of $625^{\circ} \mathrm{C} / \mathrm{sec}, \mathrm{Fe}_{3} \mathrm{C}$ particles less than $2 \mu \mathrm{m}$ in diameter would dissolve completely prior to reaching the eutectic temperature of $1271^{\circ} \mathrm{C}$. On the other hand, in case of less readily dissociable alloy carbides or intermetallic compounds involving a substitutional type solute, the lowered rate of dissolution makes constitutional liquation unavoidable, except for welding conditions producing extremely slow heating and cooling rates. Constitutional liquation was also substantiated by the work of Krantz et al. [43] and Owczarski et. al. [1].

It should also be recognized that in the actual weld, grain boundary migration also occurs. Thus, in the presence of constitutional liquation, it is probable that the moving grain boundaries will intercept these liquated regions and grain boundary penetration by the liquid phase will result. Such penetration would tend to arrest any further grain growth until the liquid phase has disappeared.

In summary, the phenomenon of constitutional liquation results in the formation of solute-rich liquid pools at temperatures well below the equilibrium solidus of the alloy, and interactions between these regions and the migrating grain boundaries could establish a microstructure sensitive to hot cracking.

\subsubsection{2: Grain Boundary Penetration Mechanism:}

The grain boundary penetration mechanism for $\mathrm{HAZ}$ liquation cracking involves the interaction of a migrating $\mathrm{HAZ}$ grain boundary with liquating matrix particles such as carbides, borides, sulphides etc. The liquation of matrix particles occurs by the process of constitutional liquation. However, constitutional liquation, a priori, is not sufficient to 
support the penetration mechanism. Since HAZ cracking is a grain boundary phenomenon, the migration of grain boundaries and interaction of these boundaries with the constituent particles are requisites of the mechanism. However, if the constituent particles are already situated at the grain boundary the above condition is not required. Since grain growth is a thermally activated process, a threshhold temperature exists above which substantial grain boundary migration is favorable. In single phase materials, the locus of this temperature generally represents the boundary of the 'true' $H A Z$. The rate of grain boundary migration increases as a function of proximity to the fusion line. In the region of the HAZ where constitutionally liquated particles are usually observed, grain boundaries are normally quite mobile and the likelyhood of interaction between a boundary and particle is quite high.

HAZ grain boundary liquation, as defined by the penetration mechanism, requires the simultaneous occurrence of constitutional liquation and grain boundary migration, again assuming that liquated species are not already at the grain boundary sites. The penetration of the boundary by the liquid at the particle-matrix interface either pins the boundary or significantly slows its rate of migration and allows uniform wetting of the boundary by the liquid. These liquid films then serve to embrittle the HAZ grain boundaries in the presence of sufficient mechanical and/or thermal induced restraint imposed during the welding process.

Many investigators have used the penetration model to explain HAZ microfissuring in a variety of alloys. Duvall and Owzarski $[1,16]$ observed the constitutional liquation of $M C$ and $M_{6} C$ carbides and related this phenomenon to $H A Z$ embrittlement in a variety of nickel-base superalloys. Savage and Krantz [43] associated HAZ hot cracking in Hastelloy $X$ with the constitutional liquation of an $M_{6} C$ carbide. Pepe and Savage [46] concluded that HAZ grain boundary liquation in 18-nickel maraging steels was the result of the constitutional liquation of titanium sulphide particles that formed along rolling bands in the base metal. In the family of austenitic stainless steels Tamura and Watanabe $[34,35]$ related embrittlement in type 347 to the liquation of niobium carbides. 
Recent studies by Thompson et. al. on alloy 718 [42] have suggested the thermodynamic feasibility of constitutional liquation reactions involving niobium-rich carbides and the kinetics of these reactions are strongly dependent on both the composition of the surrounding austenitic matrix and the weld thermal cycle. They have also reported that the cracking of the HAZ grain boundaries in this alloy is influenced by the temperature range over which the niobium-rich, grain boundary liquid solidifies during the on-cooling portion of the weld thermal cycle and the composition and temperature-dependent distribution of the liquid along the HAZ boundaries.

\subsubsection{3: Grain Boundary Segregation Mechanism:}

Despite the utility of the grain boundary penetration mechanism, HAZ liquation cracking is often encountered in materials where constitutional liquation does not, or cannot, occur. As a result, a liquation mechanism has evolved that is not dependent on the interaction of a mobile grain boundary with a liquated constituent particle. Many single-phase materials that are relatively free of intermetallic and constituent particles have exhibited susceptibility to $\mathrm{HAZ}$ liquation cracking. In general, the location of embrittled grain boundaries in the HAZ of these materials is nearly identical to that of materials which are embrittled by the penetration mechanism. As a result, a grain boundary segregation model that rationalizes the onset of grain boundary liquation in the absence of constituent particle melting has been proposed to explain $\mathrm{HAZ}$ liquation cracking.

The segregation model in its simplest form provides for solute/impurity element segregation to grain boundaries, thereby reducing the melting temperature of the boundary relative to the surrounding matrix. Above a critical temperature during the $H A Z$ thermal cycle, preferential melting occurs along these boundaries effectively embrittling a narrow region of the HAZ adjacent to the weld fusion zone. The actual mechanism by which solute/impurity atoms segregate to grain boundaries is not clear.

A possible mechanism for grain boundary segregation evokes equilibrium diffusion, 
where the segregated region is localized within a few atom diameter on either side of the boundary. This type of grain boundary segregation is driven by the free energy difference between a solute or impurity atom on a matrix site vs a grain boundary site. This segregation occurs when the solute/impurity element is highly surface active and/or exhibits a low solubility in the base metal. In many of the high performance structural steels and stainless steels, elements such as sulfur, phosphorus, carbon, antimony, tin and arsenic exhibit this behavior. The behavior of an individual element, however, is a strong function of the base metal composition, metallurgical condition and thermal history.

An equilibrium grain boundary segregation model is most tractable when one assumes that atomic rearrangement occurs at a static boundary. In the HAZ of a typical fusion weld, grain growth begins above a threshhold temperature during the on-heating portion of the thermal cycle and continues upto the local peak temperature. Under these conditions, grain boundary liquation due to equilibrium segregation then occurs once boundary motion has essentially stopped during the on-cooling portion of the thermal cycle. The HAZ embrittlement window, via this mechanism, is thus quite narrow since the grain boundary velocity must go to zero at a temperature that is sufficient to promote melting along the boundary. From a strictly theoretical basis, grain boundary liquation due to equilibrium segregation would be enhanced by moderate to slow HAZ heating and cooling rates. For the austenitic stainless steels, this is, in fact, what is normally observed; HAZ cracking is almost never observed in electron beam welds or low heat input arc welding processes.

Alternatively, it has been argued that equilibrium segregation to grain boundaries is not sufficient to support liquation in the HAZ since the segregation field is extremely localized. As a result, a boundary sweeping mechanism has been proposed. As grain boundaries migrate upon heating above the threshhold grain growth temperature, solute and/or impurity atoms are swept into the boundaries and are dragged along as grain growth proceeds. Again, elements that are surface active and/or exhibit a low solubility in the matrix would have the highest probability of being swept into and assimilated with the 
boundary. As the temperature in the HAZ increases above local grain boundary melting temperature, liquation occurs and the region of the $H A Z$ within a critical embrittlement range becomes susceptible to liquation cracking.

Duvall and Owzarski [47] have argued that grain boundary migration actually results in boundary breakaway from solute atmospheres and that the instantaneous composition of the mobile boundary reflects the local composition of the matrix through which it is moving. They provided metallographic evidence for this argument by showing ghost grain boundaries in the HAZ at the sites where breakaway occurred during initial boundary migration. Lippold [38] pointed out, however, that while some solute elements are not mobile enough to migrate with the boundary, others are extremely mobile and may move with the original boundary or be swept from the matrix as the boundary migrates. Thus, larger, slow diffusing atoms remain behind to form ghost boundaries while surface active, fast diffusing elements will move with the boundary.

More recently, Romig et. al. [48] have investigated the potential for grain boundary sweeping in simulated HAZ microstructures in Alloy 800 . Using analytical electron microscopy techniques, no clear evidence for grain boundary enrichment via a sweeping mechanism could be detected in samples heated into the liquation temperature range and then quenched in-situ. Instead, a microconstitutional liquation mechanism was detected whereby submicron $\mathrm{Ti}(\mathrm{C}, N)$ particles react with the surrounding matrix and liquate. This liquation resulted in localized wetting of the boundaries and a subsequent loss in ductility, thereby producing a crack susceptible mocrostructure.

Another mechanism for HAZ grain boundary segregation is described by the pipeline diffusion theory. Since weld solidification occurs via an epitaxial nucleation process, grain boundaries are continuous from the fusion zone across the fusion line into the HAZ. By the nature of the solidification process, partitioning of solute and impurity elements along the solidification grain boundary results in solute/impurity enrichment relative to the adjacent, epitaxial HAZ boundary. This boundary provides a diffusion pipeline for elemental 
segregation and subsequent enrichment of the HAZ boundaries. Since grain boundary diffusion is orders of magnitude faster than lattice diffusion $[49,50]$, this mechanism provides a plausible explanation for grain boundary liquation adjacent to the fusion line.

The mere occurrence of liquation reaction, either by constitutional liquation or boundary segregation induced liquation, is not sufficient to produce a crack susceptible microstructure. In order to embrittle the microstructure, it is essential that the liquid species penetrate and wet the boundary. If wetting is inefficient, liquid will exist in isolated pockets allowing substantial solid bridging along the grain boundaries. As wetting becomes more effective, boundaries will be uniformly coated with a thin liquid film. As described in earlier sections, the wetting characteristics are a function of the composition of the liquid and solid in contact, the crystal structure of the solid substrate, the relative surface free energies and the temperature.

\subsection{7: Effect of Base Metal Microstructure on Weldability}

Initial microstructure has been known to affect the HAZ microfissuring susceptibility of an alloy. The specific aspects of the microstructure that are important are (i) solution and aging treatments (ii) minor phase precipitation on the boundary (iii) grain size (iv) segregation of trace elements to the grain boundaries. These aspects are reviewed in the subsequent sections.

2.1.7.1: (i) Solution and Aging treatment: It has been shown that the HAZ microfissuring susceptibility of Inconel 718 can be controlled by the use of appropriate solution treatment. Thompson et. al. [42,51] have reported that the weldability of alloy 718 with respect to microfissuring susceptibility was affected beneficially by solution annealing and adversely by aging. Inconel 718 when welded in the fully aged condition shows excessive HAZ microfissuring [42,51]. It has also been observed [52,53] that Inconel 718 specimens given high temperature solution treatments are more susceptible to HAZ microfissuring. Gordine [52] observed in his study that low solution treatments (less than 
$1960^{\circ} \mathrm{F}$ ) before welding can reduce HAZ microfissuring. No reasons, however, have ever been advanced in the literature as to why high temperature solution treatments lead to greater microfissuring susceptibility even when grain size is within the required range.

Significant structural changes were reported in the HAZ of Udimet 700 [54] when welded in a fully heat treated condition. Specifically, maximum resistance to weld cracking both during welding and during post weld heat treatment was obtained by a two step overaging treatment. In general, alloys like Rene 41 will weld without cracking if they are solution annealed, thoroughly cleaned and special precautions taken to prevent oxygen contamination [55]. However, excessive cracking will occur if the weldments are directly age hardened. To prevent this, the weldments are reheated to the solution annealing temperature before aging [55]. Then, they can usually be age hardened without difficutly. Berry and Hughes [56] have reported that overaging Rene 41 prior to welding was the single most effective method to reduce strain age cracking. However, Rene 41 too, like most other weldable superalloys, cracks extensively when welded in peak aged condition.

2.1.7.2: (ii) Minor Phase Precipitation on the grain boundaries: Minor phases can affect the microfissuring susceptibility especially, if they are situated on the grain boundaries. The presence of phases like borides, carbides and sulphides can lead to their constitutional liquation and/or solid state dissolution enriching the boundary with elements that lower the local melting point significantly. This leads to the formation of liquid films on the boundary and subsequent microfissuring due to the restraint force exerted on the welding.

Wu and Herfert [57] by microstructural studies of Rene 41 simulated weld heat affeced zones have shown that microfissuring in the weldment was due to the presence of low melting constituent $M_{4} B_{3}$. They also reported that the dissolution of $M_{6} C$ and its subsequent reprecipitation during the weld cooling cycle as $\mathrm{M}_{23} \mathrm{C}_{6}$ on the grain boundaries led to grain boundary embrittlement and strain age cracking. In Inconel 718, the formation of liquid phases has been attributed to the constitutional liquation of $\mathrm{NbC}[16,42]$, Laves phase $[41,53,58]$ and possibly minor phases like borides $[41,59]$ or silicides [41] in 
the wrought alloys.

As discussed earlier in section 2.1.6.2.1, Duvall and Owzarski [1,16] observed the constitutional liquation of $M C$ and $M_{6} C$ carbides and related this phenomenoa to $H A Z$ embrittlement in a variety of nickel-base superalloys. Savage and Krantz [43] associated $H A Z$ hot cracking in Hastelloy $X$ with the constitutional liquation of an $M_{6} C$ carbide. Pepe and Savage [46] concluded that HAZ grain boundary liquation in 18-nickel maraging steels was the result of the constitutional liquation of titanium sulphide particles that formed along rolling bands in the base metal. In the family of austenitic stainless steels Tamura and Watanabe related HAZ embrittlement in Type 347 [34,35] to the liquation of niobium carbides. Romig et. al. [48], using analytical electron microscopy techniques have observed constitutional liquation of sub-micron Ti(C,N) particles on the grain boundaries in Alloy 800.

2.1.7.3: Effects of Trace Elements: Solution hardened alloys of the cobalt or nickelbase type should be relatively easy to weld. They have no phase changes or precipitation hardening reactions, which would normally cause problems during welding. However, because of the wide liquidus to solidus range normally present in these alloys, hot cracking and fissuring during welding is often a problem [55].

Minor elements such as sulphur in nickel-base alloys and boron in cobalt-base alloys are notorious for causing dramatic increases in cracking during welding if slightly above their specified values. In these cases, the zero ductility range is greatly increased. However, in many cases, crack sensitive heats of particular alloys appear even with all the minor elements within specification. A statistically designed factorial experiment was performed on Hastelloy $X$ by Yeniscavich and Fox [60] to understand effects of eight minor elements on cracking sensitivity of the weld heat affected zone. Cracking sensitivity was increased by high levels of $B, S, P$ and $C$. Si and $\mathrm{Mg}$ had a slightly detrimental effect, and $\mathrm{Mn}$ and $\mathrm{Zr}$ had a slightly beneficial effect. However, even for the four elements showing the greatest detrimental effects, the increase due each individual element was not large. Hence it was 
concluded by Yeniscavich and Fox that minor elements, when within normal specification limits, do not individually cause significant increases in HAZ cracking sensitivity. However, when several minor elements are present at nominally high levels, their effects are additive and significant increases in HAZ cracking sensitivity can occur.

Savage et. al. [61] have also studied the effect of minor elements on the hot cracking tendancies of Inonel 600. They found $S$ and $P$ to be extremely detrimental to all three of the cracking parameters viz. the total crack length, maximum crack length and the average crack length. They attributed the effect of $P$ and $S$ to a high degree of segregation, and subsequent reduction in the effective solidus and liquidus coupled with a decrease in the solid-liquid interfacial energy, which permitted wetting of the grain and subgrain boundaries and subsequent formation of grain boundary films. Both $\mathrm{Mn}$ and Si were found by them to reduce the detrimental effect of $S$, at least in part as a result of increase in the solid-liquid interfacial energy. However, a significant Si-Mn interaction effect indicated that these elements were not as effective at reducing the effect of $S$ when both were present together. In their investigation, it was also observed that a S-P interaction increased the detrimental effect of the two elements when both were present in combinations.

2.1.7.4: Grain size: Aplett and Pellini [62] were one of the first investigators to experimentally observe that a fine grain size in the base metal reduced HAZ microfissuring. Subsequently, various studies have shown that in Inconel 718 , fine grain sizes promote resistance to HAZ microfissuring. In the 70's a grain size less than ASTM \# 6 was used in wrought alloy 718 to alleviate the problem of HAZ microfissuring. Thompson et. al. [63] have found that microfissuring susceptibility of wrought Inconel 718 was linearly dependent upon grain size. This may be because at finer grain sizes a greater grain boundary area is available to accommodate the strains devoloped during welding. High preweld solution treatment temperatures that result in larger grain size have been reported to increase the tendency to microfissuring.

The effect of grain size to microfissuring could be related to both liquid distribution 
and grain boundary sliding. A large grain size would lead to a thicker liquid layer than a small grain size if same volume of the liquid is present in both cases. A larger volume fraction of inter-granular liquid could increase the temperature range and the time duration during which liquid wets the grain boundary faces under non-equilibrium freezing conditions. This would lead to an increased microfissuring susceptibility. A large grain size would also cause a longer interface sliding length, which would lead to larger stress concentrations, larger strains at grain boundary triple points and thus increased crack intiation due to grain boundary sliding.

The extent of grain coarsening that occurs in the fusion zone may also be important. Thamburaj et. al. [64] have reported that a coarse grain fusion zone makes the alloy very susceptible to HAZ microfissuring. It is believed that a coarse grain fusion zone resists plastic deformation causing welding strains to concentrate in the HAZ.

Sheet materials with small thickness-to grain diameter are reported to be more susceptible to HAZ microfissuring [65]. In the case of Hastelloy $X$ and Hastelloy $S$, cracking is most severe when thickness-to-grain diameter ratio decreases to a value of 4 or less. The increased microfissuring tendancy in low thickness-to-grain diameter ratio in materials is a manifestation of the greater ease of inter-granular crack propagation, once the HAZ hot cracks originates at a liquid grain boundary.

\subsection{THE 900 SERIES SUPERALLOYS}

\subsubsection{Introduction}

The 900 series superalloys, based on the Fe-Ni-Co system, represent a new class of aerospace materials designed for room and elevated temperature strength and a low coefficient of thermal expansion [66-70]. Iron-nickel-cobalt alloys have long been used in instrumentation and electronic applications because of their unique physical properties. These alloys are characterized by a low coefficient of thermal expansion upto a magnetic transition temperature, the Curie temperature. The anamolous low expansion of 
these alloys is associated with the changes in volume magnetostriction with temperature. These volume changes oppose the normal thermal expansion. Low expansion alloys have traditionally been used in applications where invariance of dimensions with small change in temperature is required or where expansion over a range of temperatures matched to that of a different material has been necessary. In the mid 1960's Huntington Alloys Inc. modified the iron-nickel-cobalt system by adding niobium, titanium and aluminum thus making a precipitation hardenable alloy [66]. These additions, normally less than $7 \mathrm{wt}$ $\%$ in total, boosted the room temperature strength of the alloy by three fold by the age hardening mechanism. The result was a new class of aerospace material: the 900 series low expansion superalloys. These alloys are fully austenitic and yet are ferromagnetic at room temperature. With proper solutionizing and aging heat treatments, the 900 series superalloys can develop excellent short term tensile properties comparable to that of Inconel $718[67]$.

Most superalloys have limitations in thermal fatigue and thermal shock environments partly due to their high mean coefficient of thermal expansion which increases with temperature over useful service ranges. The values of mean coefficient of thermal expansion of the 900 series alloys are well below those of alternate commercial superalloys, being approximately one half that of the nickel base superalloy Inconel 718 at the inflection temperature $[67,68,70]$. Hence, the 900 series alloys owing to their low expansion property have superior resistance to thermal fatigue. Incoloy 903 has three times the thermal fatigue life of Inconel 718 . The 900 series alloys are also highly resistant to high pressure hydrogen embrittlement [67]. Low expansion is of interest in gas turbines to control tip clearance over the temperature range of the operation of the engine, and is also attractive for shafts and other components of the engine.

Incoloys 903, 907 and 909 are the most important alloys among the 900 series low expansion alloys. Currently, Incoloy 903 is the most widely used alloy of this class. The aerospace industry is presently expanding the application of Incoloy 903 as well as the 
next generation low expansion alloys, 907 and 909.

\subsubsection{Physical Metallurgy}

Incoloy 903 is a precipitation hardenable superalloy deriving its strength from the precipitation of coherent, fine spherical $\mathrm{Ni}_{3}(\mathrm{Al}, \mathrm{Ti}, \mathrm{Nb}) \gamma^{\prime}$ phase [66-70]. $\gamma^{\prime}$ forms homogeneously in the matrix with an ordered, fcc structure. Aging at a higher temperature can sometimes lead to the formation of needle shaped $\mathrm{Ni}_{3} \mathrm{Nb}$ delta phase [68]. Incoloy 903 is commercially utilized in the peak aged condition. Incoloy 907 differs from Incoloy 903 in terms of its decreased aluminum content and increased niobium and titanium content. Incoloy 907 is used in an overaged condition for acceptable notch rupture properties. Overaging promotes a complex microstructure comprised of inter- and intragranular $\mathrm{Ni}_{3}(\mathrm{Al}, \mathrm{Ti}, \mathrm{Nb})$ epsilon phase or epsilon double prime and gamma prime $[70,71]$. Incoloy 909 , the most recent of the low expansion alloys differs compositionally from Incoloy 907 primarily in its four fold increase in silicon content, a modification that markedly improves notch rupture properties and allows in eliminating microstructural anisotropy. Microstructural studies of Incoloy 909 have revealed a variety of phases on aging, including epsilon phase, $\mathrm{Fe}_{2} \mathrm{Nb}$ Laves phase (hexagonal and cubic) and a cubic silicide, $\mathrm{Ni}_{1} 6 \mathrm{Nb}_{6} \mathrm{Si}_{7}$ (G phase) [72].

In addition to the phases that occur on aging, the presence of carbon in the 900 series alloys promotes the formation of niobium-rich cubic MC carbides during both ingot solidification and in solid state during thermomechanical processing and heat treatment. Coarse niobium-rich MC carbides/carbonitrides are present within the gamma grains, typically aligned in the rolling direction. Cuboidal titanium-rich carbonitrides, often observed at the center of the coarser carbides, serve as nucleation sites during ingot solidification. Depending on the thermomechanical processing and heat treatment, fine, niobium rich carbides may also form in the solid state at the gamma grain boundaries. As expected, the lower carbon levels in Incoloy 909 result in the presence of fewer carbides relative to 
Incoloys 907 and 903.

High tensile strengths in Incoloy 903 can be obtained using a conventional solution heat treatment $[982 \mathrm{C} / 1 \mathrm{~h}$, air cool followed by aging at $718 \mathrm{C}$ for $8 \mathrm{hrs}$, furnace cool at $50 \mathrm{C} / \mathrm{hr}$ to $621 \mathrm{C}$ and aging for $8 \mathrm{hrs}$, air cool] [73]. This heat treatment cycle promotes a fine recrystallized gamma grain structure and the uniform precipitation of $\mathrm{Ni}_{3}(\mathrm{Al}, \mathrm{Ti}, \mathrm{Nb})$ gamma prime $\left(\gamma^{\prime}\right)$. However, optimum high temperature mechanical properties in Incoloy 903 are obtained by controlled thermomechanical processing and heat treatment. More specifically, Incoloy 903 is thermomechanically processed to improve notch-rupture ductility. A typical processing schedule involves warm working followed by a moderate anneal at $840^{\circ} \mathrm{C} / 1 \mathrm{~h}$, which is below recrystallization temperature and above the $\gamma^{\prime}$ solvus, and an aging treatment of $760^{\circ} \mathrm{C}$ for 5 hours followed by furnace cool to $650^{\circ} \mathrm{C}$ and further aging for an hour. This thermomechanical processing induces a necklace grain structure in the alloy, where large warm worked grains are surrounded by fine recrystallized grains. The aging schedule is aimed at developing a duplex $\gamma^{\prime}$ particle size. The necklace structure and duplex $\gamma^{\prime}$ particle size has been observed to give superior mechanical properties. In principle, alloy 903 can be hot worked in the temperature range $815^{\circ} \mathrm{C}$ to $1120^{\circ} \mathrm{C}$ but for superior stress-rupture properties, the final heat treatment temperature should be a maximum of $925^{\circ} \mathrm{C}$ with a minimum of $25 \%$ reduction at $870^{\circ} \mathrm{C}$ or lower [73]. This processing sequence results in a highly textured microstructure and corresponding anisotropic mechanical properties. Due to the retention of the warm worked structure, the alloy is notch brittle in the transverse direction. The transverse notch-rupture strength is only a third of the longitudinal strength [67]. Incoloy 903 does retain good yield and tensile strength upto $650^{\circ} \mathrm{C}$. Beyond this temperature the age hardening phase begins to overage rather rapidly.

The specific expansion rate and the Curie temperature of iron-nickel-cobalt alloys is a function of $\mathrm{Ni} / \mathrm{Fe}$ or $(\mathrm{Ni}+\mathrm{Co}) / \mathrm{Fe}$ ratio. The main effect of cobalt when substituted for nickel is to raise the inflection temperature and thereby extend the range over which low 
expansion occurs. One major alloying addition to iron-base, nickel-iron base and nickel base superalloys is chromium, whose primary function is to provide oxidation resistance. However, the 900 series superalloys are chromium free. Studies [68] indicate that over one percent of any of chromium, molybdenum and tungsten have an adverse effect of drastically increasing the mean coefficient of thermal expansion of iron-nickel-cobalt alloys. Hence, these elements are completely eliminated from the 900 series alloys. Because of the elimination of chromium, the time dependent properties, particularly notch rupture strength, can be greatly affected by a mechanism of stress corrosion cracking aided by oxidation referred to generally as stress accelarated grain boundary oxidation (SAGBO) [67]. It has been observed that thermomechanical processing could to some extent offset SAGBO and provide acceptable notch rupture strength [70]. A lowered aluminum content has been observed to improve the resistance to oxidation/stress cracking mechanism. Increased niobium and titanium contents have been found to compensate for the adverse effect on tensile properties due to lowered aluminum. These observations have led to the development of Incoloy 907. The aluminum content in Incoloy 907 was reduced to 0.03 wt \% from 0.9 wt \% in Incoloy 903 and niobium content was increased to 4.7 wt \% from 3.0 wt $\%$ in Incoloy 903. However Incoloy 907 also requires thermomechanical processing and retention of a hot worked structure for adequate notch-rupture strength and hence is microstructurally anisotropic like Incoloy $903[70,71]$. Incoloy 909 is the most recent of the low expansion superalloys. As mentioned earlier, alloy 909 has higher silicon as opposed to alloy 907 as this was observed to impart superior resistance to SAGBO apart from enabling to eliminate microstructural anisotropy [72]. Incoloy 909 is given a high temperature solution treatment prior to aging to produce a fully recrystallized equiaxed grain size thus eliminating microstructural anisotropy.

\subsubsection{Weldability of 900 Series Superalloys}

Metallurgical problems associated with the welding of $\mathrm{Ni}$ and its alloys include weld 
porosity, embrittlement by sulphur and other contaminants and loss of corrosion resistance due to the formation of intergranular precipitates. Hot shortness of nickel and nickel alloys may be caused by contamination with $S, P$, lead and a number of low melting elements such as bismuth [7]. These contaminants form intergranular films which cause severe embrittlement and cracking at elevated temperatures [7]. Hot cracking of weld metal may result from such contamination, but more frequently it occurs in the HAZ and is caused by the penetration of contaminants from from the weld metal surface [7]. The $\gamma^{\prime}$ precipitation kinetics in nickel-base superalloys affects the strain age cracking susceptibility of the alloy during post weld heat treatment. The $\gamma^{\prime}$ precipitation reaction is so rapid in many superalloys that it can form during the HAZ cooling cycle. It is now well known that if $\gamma^{\prime}$ precipitation occurs in the HAZ during the weld cooling cycle or rapidly during subsequent reheating, strain age cracking results. Alloy 718 is generally considered to have good weldability owing to the sluggish $\gamma^{\prime \prime}$ precipitation kinetics [64].

The status of welding technology for nickel-base superalloys is shown in fig 2.21 [55]. Those with low $\mathrm{Al}$ and $\mathrm{Ti}$ contents, shown below the dashed line in the figure are readily weldable. However, as combined $\mathrm{Al}$ and $\mathrm{Ti}$ is increased, welding becomes more difficult. Alloys like Rene 41 and Waspaloy are borderline; they weld with relatively little difficulty but some times crack during post weld heat treatment [55]. Based on the criterion shown in fig 2.21 the 900 series alloys may be considered readily weldable.

The weldability of niobium containing superalloys is defined primarily in terms of susceptibility to heat affected zone liquation cracking, and fusion zone solidification cracking. Some authors [74] refer to fusion zone liquation cracking as the cracking that occurs in the underlying pass of a multipass weldment. In this author's view, this terminology is redundant and actually adds to the further confusion to an already chaotic terminology as both kinds of cracking are thought to occur during the liquid film stage of solidification. The fundamental difference that an underlying pass is a cast structure as opposed to the initial base metal structure is not obscure to any reader. It appears more appropriate, 


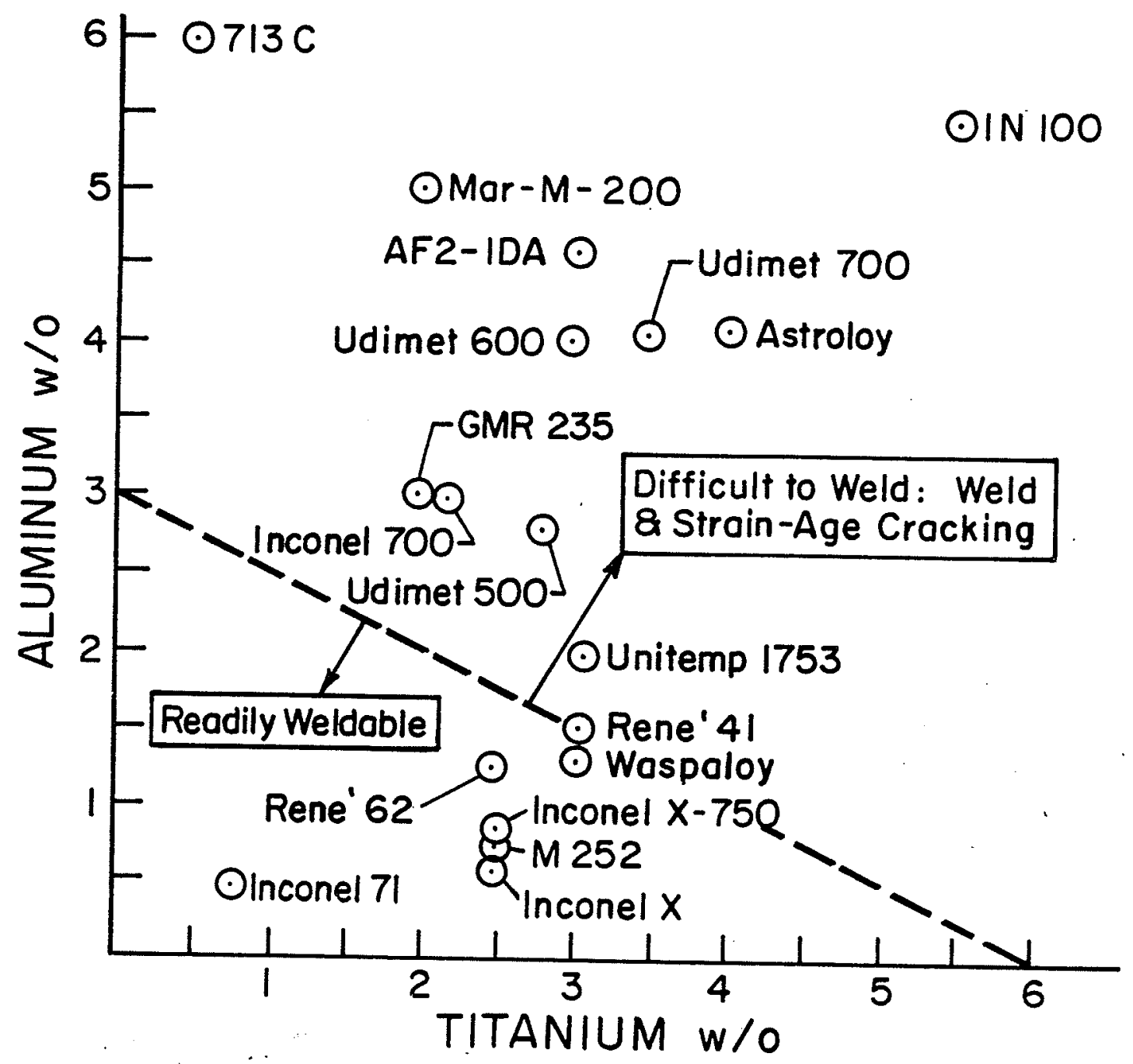

Fig. 2.21 : Seperotion between $\gamma^{\prime}$ strengthened olloys that ore difficult to weld and those readily weldable [55]. 
as discussed earlier, for the definitions to be based on the operating mechanism that is responsible for cracking.

The sensitivity of a particular alloy to solidification and $H A Z$ liquation cracking is dependent primarily upon the alloy composition, and the stress state in the weld although HAZ cracking may also be influenced by the base metal microstructural condition. Improvements in the high temperature mechanical behavior of the 900 series alloys through compositional tailoring and thermomechanical processing makes these alloys more amenable to widespread commercial usage. However, the nature of the compositional changes required to achieve these property improvements, in particular, increases in niobium and silicon, might be expected to alter the susceptibility of these alloys to cracking during welding. During the past decade, numerous investigators have shown that fully austenitic iron and nickel-base alloys that contain niobium and titanium can be highly susceptible to liquation cracking. In Nb- and Ti-containing alloys, the liquation phenomena, which seems a prerequisite for cracking, has been attributed to several different mechanisms including the constitutional liquation (eutectic liquation) of Nb- and Ti- rich carbides $[16,42,53,58,75]$ and the "sweeping up" of free titanium residual in the matrix from the dissolution of Ti-rich carbides [38] and pipe line diffusion of niobium from niobium enriched solidification boundaries in the fusion zone that are continuous across the fusion line into the HAZ [76]. Earlier studies have shown evidence of intergranular cracking and incipient melting at the grain boundaries. Hence, based on the comparitively high level of niobium and titanium in the 900 series alloys, liquation cracking may be anticipated. The weldability of 900 series alloys have been studied to a limited extent by spot-varestraint tests and SEM [77], spot varestraint test and mini-varestraint test and SEM [78] and by gleeble hot ductility testing [79]. A brief discussion of the results of these investigations follows.

Baeslack et al. [77] studied the weldability of Incoloy 903 by spot varestraint test and SEM. They had used a commercial heat of Incoloy 903 plate given a final solution 
treatment for 1 hour at $940^{\circ} \mathrm{C}$. Initial microstructural characterization by SEM revealed a near equiaxed austenite grain structure with a bimodal distribution of carbides throughout the microstructure. Quantitative analysis of $H A Z$ liquation cracking was performed by spot varestraint testing on all base metal specimens. Since, weld metal liquation cracking occurs in the underlying pass of a multiple pass weldment, a study of weld metal liquation cracking was done by testing double welded specimens. Microstructural characterization of the weld and HAZ was done by SEM/EDS.

The HAZ liquation cracks were found to propagate approximately perpendicular to the fusion line with no extension into the fusion zone. Quantitative cracking susceptibility of the weld metal was observed to be significantly greater than that of the solution annealed base metal. A rather unpalatable result obtained in their investigation is the observation of lower total crack length in the HAZ of single welded specimens as compared with the double welded specimens.

Baeslack et al. [77] concluded by microstructural characterization using SEM/EDS that extensive regions of partial melting observed in both base metal and weld metal HAZ regions were due to the liquation attributed primarily to the constitutional liquation of niobium-rich carbides in the base metal and the Laves phase in the weld metal. According to these authors, both eutectic reactions (the gamma/Laves and the gamma/carbide) resulted in the formation of low melting austenite/Laves eutectic at the grain boundaries, which served as a precursor to liquation cracking. They also attributed the greater liquation cracking sensitivity of the weld metal to a lower eutectic reaction temperature of the Laves phase versus the niobium-rich carbides, a coarser austenite grain size and a less tortuous intergranular crack path. Despite numerous shortcomings in the experimentation and interpretation, this first work does indicate the fact that Incoloy 903 is prone to HAZ liquation cracking.

The next published work relates to the gleeble hot ductility testing [79] and spot varestraint weldability testing of the 900 series alloys, Incoloys 903 and 909 . Metallo- 
graphic and fractographic examination of the test samples revealed that loss of ductility and subsequent cracking at high temperatures resulted from the presence of low melting liquid films at the austenite grain boundaries. It was concluded that the constitutional liquation of niobium-rich carbides in Incoloy 903 and niobium and silicon-rich Laves and/or $G$ phase in Incoloy 909 provided the principle source of the liquid. Hot ductility test results, interpreted using conventional techniques, were found to be inconsistent with the predictions of cracking susceptibility obtained with the spot varestraint test, with a higher susceptibility of Incoloy 903 in the former and Incoloy 909 in the later.

The most recent study of the weldability of the high strength, low expansion superalloys was undertaken by Ernst et. al. [78]. They investigated the HAZ liquation cracking and weld metal solidification cracking in Incoloys 903, 907 and 909 using the spot and mini varestraint weldability tests and microstructural characterization by SEM/EDS. All their experimentation was carried out on alloys exhibiting comparable recrystallized austenite microstructures. The liquation nature of the cracking processes was studied by fractographic analysis of the crack surfaces by SEM.

By comparing the average total crack length versus the augmented strain levels imposed during the testing, Ernst et al [78] observed Incoloy 903 exhibited the lowest HAZ cracking susceptibility, particularly at higher augmented strains, followed in order of increasing susceptibility by Incoloys 907 and 909 . They also observed that the total number of cracks correlated well with the total crack length while maximum crack length remained constant without showing any clear trend.

They speculated that HAZ liquation cracking resulted from the presence of low melting Laves phase/gamma eutectic liquid that was enriched in niobium, titanium and silicon, based on microstructural examination of spot varestraint test samples via optical and SEM/EDS. This liquid originated in part from the constitutional liquation of niobiumrich carbides in Incoloys 903 and 907 and G phase and/or Laves phase in Incoloy 909. Fractographic analysis of the $H A Z$ liquation crack surfaces showed a fine wavy pattern 
on the grain faces and evidence of melting along grain boundaries and at triple points, indicating the presence of a thin liquid film prior to cracking.

The relative fusion zone solidification cracking susceptibility as determined from mini varestraint weldability testing was found to be $903<907<909$. The weld solidification cracking susceptibility originated from the partitioning of niobium, titanium and silicon to grain and solidification substructure boundaries during solidification and formation of a low melting point terminal gamma/Laves eutectic constituent. The relative susceptibility to fusion zone cracking in the alloys was related to the effects of increased niobium, titanium and silicon levels on the quantity and freezing temperature of the terminal eutectic liquid. The greater the niobium, titanium and silicon level, the greater is the volume fraction of the terminal eutectic liquid. They also concluded based on their experimental observations that the volume of the terminal eutectic liquid was below the critical value required for crack healing. All the three investigations [77-79] make similar emphatic conclusions regarding the phase changes occurring on the liquated $H A Z$ boundaries and in the fusion zone despite any definitive phase analysis being performed by either electron diffraction or x-ray diffractometry.

\subsection{SCOPE AND NATURE OF THE PRESENT INVESTIGATION}

As discussed in the previous sections, no comprehensive study using analytical electron microscopic techniques exists in open literature on the effect of initial microstructure on the weldability and nature of fusion zone and heat affected zone microstructures in the 900 series superalloys. The objective of the present study was to gain a comprehensive understanding of the physical metallurgy of welding of Incoloy 903 . Hence, the primary focus of the present investigation was a thorough microstructural characterization of the various samples using optical microscopy and image analysis, $x$-ray diffraction, scanning $x$-ray microanalysis and $x$-ray mapping and AEM. This was performed to give an understanding of the following aspects: 
1. Preweld material:

(i) the nature of grain orientation and initial grain size

(ii) the presence and the location of insolubles

(iii) the composition and lattice parameter of the matrix, large insolubles and any other phases

(iv) the morphology, crystal structure, lattice parameter, composition and site of location of minor phases

2. As-welded material

(i) the nature of the fusion zone and microsegregation pattern in the fusion zone

(ii) the nature of the interdendritic liquid

(iii) the heat affected zone microstructures

(iv) the morphology and extent of microfissuring and other features in the HAZ, the occurrence/non-occurrence of constitutional liquation in the $\mathrm{HAZ}$ and the phases responsible for such liquation reactions.

(v) the crystal structure, lattice parameter, composition and site of location of interdentritic phases, minor phases in HAZ, and of the phases extracted from microfissures.

The salient features of the experimentation are outlined in the subsequent paragraphs.

The forging conditions were kept invariant and heat treatments were utilized to produce a variety of initial microstructures. The specific conditions investigated were (table 2.1) as-forged material, as-forged and solution treated at $843^{\circ} \mathrm{C}$ for an hour (forged and solution treated ring), as-forged and solution treated at $925^{\circ} \mathrm{C}$ for an hour, as-forged and solution treated at $950^{\circ} \mathrm{C}$ for an hour, as-forged and solution treated at $975^{\circ} \mathrm{C}$ for an hour, forged and solution treated ring further solution treated at $1100^{\circ} \mathrm{C}$ for various lengths of time.

The experimental studies on HAZ microstructural characterization can be classified under three major categories. The first category pertained to the observations on HAZ microfissuring in the forged and solution treated ring where (a) most of the grains observed 


\section{Table 2.1: Microstructural Conditions Investigated}

Thermomechanical Microstructure Precipitates and Insolubles
Processing

As-forged alloy

Forged and solution

treated ring

As-forged alloy solution treated at $925^{\circ} \mathrm{C} / 1 \mathrm{~h}$

As-forged alloy solution treated at $950^{\circ} \mathrm{C} / 1 \mathrm{~h}$

As-forged alloy solution treated at $975^{\circ} \mathrm{C} / 1 \mathrm{~h}$

Solution treated at $1100^{\circ} \mathrm{C}$ for $1 / 2$ to $8 \mathrm{~h}$
Large warm worked grains outlined by fine recrystallized grains

Large warm worked grains outlined by fine recrystallized grains

Large warm worked grains outlined by fine recrystallized grains

Partially recrystallized grains

Large primary carbides pinned by carbides many fine MC carbides Straight recrystallized Primary carbides Large primary carbides MNP phosphides fine MC carbides

Large primary carbides MNP phosphides fine $M C$ carbides

Large primary carbides MNP phosphides fine $M C$ carbides Large primary carbides few MNP phosphides many fine $M C$ carbides grains 
were primarily large warm worked grains, i.e the ring samples on which the bead on plate welds were performed approximately along a constant diameter (under case $A$ welding conditions along a straight line on a $8-10 \mathrm{~cm}$ section of the ring perpendicular to the radius of the ring) and (b) bead on plate welds performed on the ring based on some of the welding conditions obtained according to a factorial design (case B welding conditions) but only in the cases where the base metal microstructure contained significant amount of fine recrystallized grains as well as warm worked grains*. Further details of the weiding conditions are provided in chapter 3 .

During the course of this investigation, an interesting relationship was observed between the equilibration mode of the liquid films that formed on HAZ grain boundaries and the microfissuring tendency; these were observed to be related to the mean grain boundary curvature. This was one of the prime reasons for microstructural studies on the bead on plate welds made on forged and solution treated ring using the factorial design, where the opportunity existed to investigate the effect of grain boundary curvature and its relationship to HAZ microfissuring. The same phenomenon was observed, though sporadically in the case $A$ electron beam welds of the forged and solution treated samples investigated first, obviously due to the fact that less than $5 \%$ of the grains were fine recrystallized ones with substantial grain boundary curvature. Furthermore, if the case $B$ welds made on forged and solution treated ring were not examined, the importance of controlled thermo-mechanical processing in obtaining good weldability would not have been appreciated.

The second part involved a study of HAZ microstructures of the bead on plate welds

* The same characterization could have been performed by making bead on plate case $A$ welds on forged and solution treated ring in a parallel direction. However, the samples from case $B$ conditions were used only because further research on these samples is underway to relate the heat input and weld shape to the occurrence of grain boundary liquid film migration. 
performed on the as-forged samples solution treated for one hour at $975^{\circ} \mathrm{C}$. The third category pertains to the observations on the HAZ microstructures on bead on plate electron beam welds performed on the forged and solution treated ring samples further solution treated at $1100^{\circ} \mathrm{C}$ in the time range of $1 / 2$ to 8 hours. The electron beam welding in both these cases was performed under case $A$ conditions.

Since, the primary focus of the present investigation was to understand the mechanism(s) of HAZ microfissuring in Incoloy 903 by relating the initial microstructure to the $\mathrm{HAZ}$ microstructure, no effort was expended in quantifying the extent of microfissuring in terms of either total crack length or total number of cracks or average crack length. However, the HAZ microfissuring susceptibility was examined qualitatively. This investigation completely characterizes the weld metal and $\mathrm{HAZ}$ microstructure and cracking morphologies thereby giving an insight in the metallurgical mechanisms responsible for the liquation and cracking phenomena during electron beam welding of thermomechanically processed Incoloy 903. 


\section{Chapter 3}

\section{EXPERIMENTAL PROCEDURES AND TECHNIQUES}

\section{1: MATERIALS AND PROCESSING}

A $15.2 \mathrm{~cm}$ diameter extruded billet of Incoloy 903 was thermo-mechanically processed by open die hammer forging to obtain the as-forged alloy. The billet was first forged to a $9.2 \mathrm{~cm}$ square cross section from a temperature of $1121^{\circ} \mathrm{C}$ by reductions of $1.3 \mathrm{~cm}$ in each step of forging. One intermediate reheat to $1121^{\circ} \mathrm{C}$ was used during the process. The work piece was then reheated to $927^{\circ} \mathrm{C}$ and further forged in two equal steps to a $7.6 \mathrm{~cm}$ square cross section with an intermediate reheat to $927^{\circ} \mathrm{C}$. The finishing temperature of the work piece was about $816^{\circ} \mathrm{C}$. No further solution treatment was given to the material at this stage. Samples cut from this alloy are referred to as the "as-forged" specimens in all subsequent discussion. Some sections of the "as-forged" alloy were given solution treatments at $925^{\circ} \mathrm{C} / 1 \mathrm{~h}, 950^{\circ} \mathrm{C} / 1 \mathrm{~h}$ and $975^{\circ} \mathrm{C} / 1 \mathrm{~h}$.

Another extruded billet of alloy 903 of $11.4 \mathrm{~cm}$ length and $15.24 \mathrm{~cm}$ diameter was soaked for one hour at $1095^{\circ} \mathrm{C}$ and upset forged, punched and sheared into a $6.4 \mathrm{~cm}$ thick ring of $7.6 \mathrm{~cm}$ internal diameter. The ring was then hot forged in five stages into a $4.6 \mathrm{~cm}$ thick ring of $26.7 \mathrm{~cm}$ outer diameter and $19.4 \mathrm{~cm}$ internal diameter. Preceding every stage, the temperature of the work piece was raised to $840^{\circ} \mathrm{C}$ by soaking it for a short time. In every stage of forging the ring was also hot rolled via rollers in a circumferential direction so as to obtain the requisite dimensions. The ring was given a solution treatment of one hour at $840^{\circ} \mathrm{C}$ following the final stage of processing. Since the material has primarily been forged it is referred to as the "forged and solution treated" ring in all subsequent 
discussion. The bulk chemical composition of the alloy in wt \% (mill analysis) as obtained by the atomic absorption technique was as follows:

Table 3.1(a): Chemical Composition of Incoloy 903

[Extruded Billet]

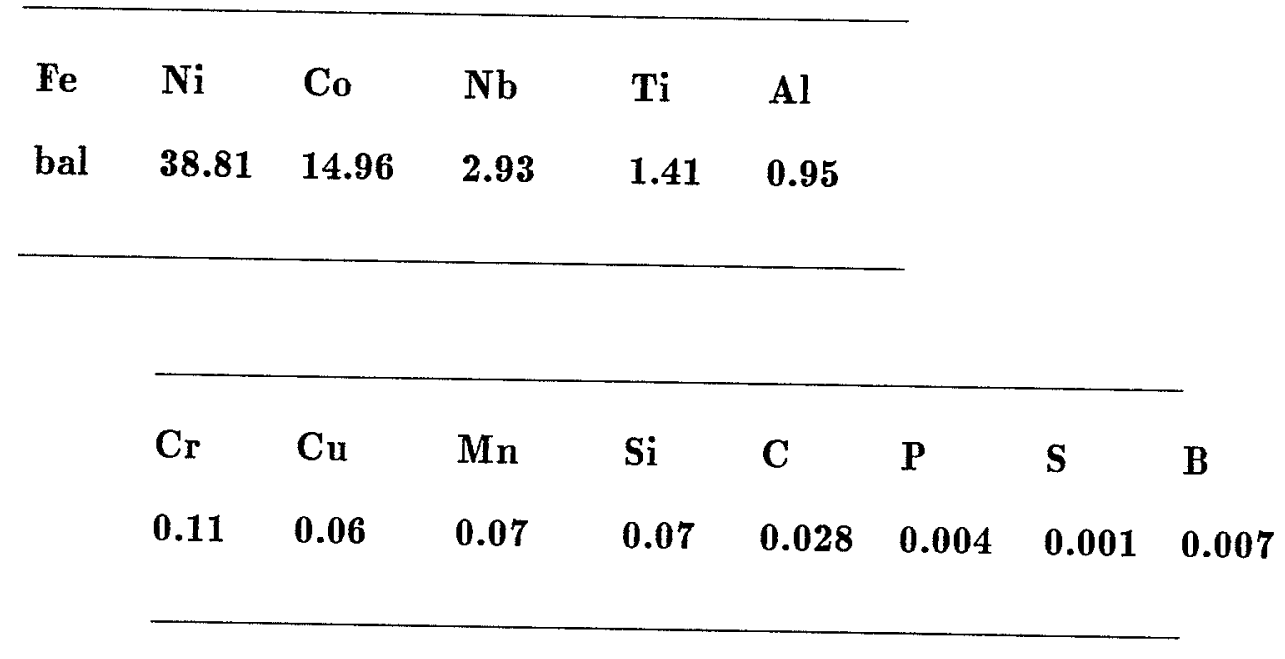

Table 3.1(b): Chemical Composition of Incoloy 903

[Forged and Solution Treated Ring]

\begin{tabular}{llllll}
\hline Fe & Ni & Co & Nb & Ti & Al \\
bal & 38.39 & 14.93 & 2.95 & 1.35 & 0.95 \\
\hline
\end{tabular}

\begin{tabular}{llllllll}
\hline Cr & Cu & Mn & Si & C & P & S & B \\
0.21 & 0.11 & 0.07 & 0.15 & 0.02 & 0.006 & 0.001 & 0.008 \\
\end{tabular}


The specimens of the alloy after various thermo-mechanical treatments viz. (a) "forged and solution treated" ring (b) "as-forged" billet (c) "as-forged" billet solution treated at $925^{\circ} \mathrm{C} / 1 \mathrm{~h}(\mathrm{~d})$ "as-forged" billet solution treated at $950^{\circ} \mathrm{C} / 1 \mathrm{~h}$ and (e) "asforged" billet solution treated at $975^{\circ} \mathrm{C} / 1 \mathrm{~h}$ were investigated by optical microscopy, $X$-ray diffraction, SEM and TEM/AEM to ascertain the nature of grain size, insolubles and precipitate distribution. A Nikon optical microscope, a JEOL JXA 840 scanning $X$-ray microanalyzer and a JEOL 2000 FX STEM were used for this purpose.

Some samples of the "as-forged" alloy solution treated at $975^{\circ} \mathrm{C} / 1 \mathrm{~h}$ and further aged at $850^{\circ} \mathrm{C}$ for three hours were studied to observe the precipitation behavior. Some samples of the forged and solution treated ring were further solution treated at $1100^{\circ} \mathrm{C}$ for $1 / 2 h, 1 h, 2 h, 4 h$ and $8 h$, respectively and were used to examine the effect of high temperature solution treatments on the weldability of Incoloy 903. Apart from the treatments described, a few samples of the forged and solution treated ring were cold rolled by $90 \%$ and were then solution treated for an hour at $1100^{\circ} \mathrm{C}$ and then water quenched. Samples from this lot were then aged at $800^{\circ} \mathrm{C}$ for varying times. Few other samples were also aged for long times in the temperature range $750^{\circ} \mathrm{C}-850^{\circ} \mathrm{C}$.

\section{2: ELECTRON BEAM WELDING}

Bead on plate electron beam welds were made on sections $1.5 \mathrm{~cm}$ thick using a Sciaky mark VII electron beam welding machine with point focus and at a working distance of $38.1 \mathrm{~cm}$. The electron beam had an accelerating voltage of $44 \mathrm{kV}$ and a current of $45 \mathrm{~mA}$. The welding speed was $152.4 \mathrm{~cm} / \mathrm{min}$. Electron beam welds were made on as-forged alloy, forged and solution treated ring, as-forged samples solution treated for an hour at $925^{\circ} \mathrm{C}$, $950^{\circ} \mathrm{C}$ and $975^{\circ} \mathrm{C}$, and the samples solution treated for varying times at $1100^{\circ} \mathrm{C}$ with the same constant welding conditions outlined above. Welds made with these electron beam welding parameters are henceforth referred to as case $A$ welds. These welding conditions lead to extensive $H A Z$ microfissuring in the forged and solution treated ring of Incoloy 903 
$[80,81]$. They were specifically chosen as the aim of the present investigation was to gain an understanding of the metallurgical causes for HAZ microfissuring as well as to study the effect of initial microstructure on HAZ microfissuring during electron beam welding of Incoloy 903.

As would be discussed in the results section, the microstructure of the forged and solution treated ring consisted of a duplex grain distribution where warm worked grains were outlined by fine recrystallized grains. However, the proportion of fine recrystallized grains to warm worked grains varied considerably from the edge to the center of the ring. Case $A$ welds were made approximately along a constant diameter i.e., along a straight line on a $3-4$ inch section of the ring perpendicular to the radius of the ring. This was done to assure a constant microstructure. The base metal microstructure of these welds contained $95 \%$ large warm woked grains, the rest $5 \%$ or less being fine recrystallized grains.

Bead on plate electron beam welds were also made on sections $1.5 \mathrm{~cm}$ thick of the forged and solution treated ring using fractional factorial experimental parameters shown in table 3.2. The following parameter and range of values were used:

Table 3.2: Fractional Factorial Experimental Parameters used for Electron Beam Welding

\begin{tabular}{lllll} 
Heat Input & Travel Speed & Voltage & Current & Working distance \\
$\mathrm{J} / \mathrm{cm}$ & $\mathrm{cm} / \mathrm{min}$ & $\mathrm{Kv}$ & $\mathrm{ma}$ & $\mathrm{cm}$ \\
$787-2756$ & $51-152$ & $35-55$ & $19-100$ & $25-64$ \\
\hline
\end{tabular}

The first twelve experiments examined a range of speed values while the second block of twelve experiments examined a range of heat input values. Each block of twelve experiments were run on one section of the ring using sharp focus on the top surface and 
no beam oscillation.

Three metallographic sections were cut from each of the twenty four welds and examined after the beam travel distances of $1.25 \mathrm{~cm}, 2.5 \mathrm{~cm}$ and $3.75 \mathrm{~cm}$. Preliminary optical microscopic examination of the HAZ microstructures indicated that some of the samples contained significant amount of both fine recrystallized grains and large elongated warm worked grains. In all subsequent discussion, no details are being provided about the specific welding parameters used and all the twenty four welds from this block of experiments are referred to as case $B$ welds. While the initial aim of using the fractional factorial experimental parameters was to understand the effect of electron beam welding parameters on $\mathrm{HAZ}$ microfissuring $[80,81]$ the results presented in this dissertation address only the microstructural characterization of the $\mathrm{HAZ}$ of some of the case B welds, in particular, where the base metal microstructure consisted of significant proportion of both large warm worked grains and fine recrystallized grains. The results of the effect of electron beam welding parameters on HAZ microfissuring are reported elsewhere [81]. Efforts are also underway to understand the effect of heat input on grain boundary liquid film migration of the liquated fine recrystallized grains and the findings will be published shortly.

While electron beam welding parameters and the shape of the weld pool have a significant effect on the microfissuring susceptibility [81], it would be shown in chapter 4 that the mean grain boundary curvature does have a significant influence on the occurrence of $\mathrm{HAZ}$ microfissures even under any constant electron beam welding parameters, provided that the nature of the liquid film formed on the grain boundaries satisfy certain conditions. Microstructural characterization of case $B$ welds were undertaken in order to investigate the effect of mean grain boundary curvature and it's relationship to HAZ microfissuring susceptibility. Some of the samples from the case B welds provided an ideal opportunity to examine and relate the microfissuring tendency to the nature of grain boundaries as they contained both fine recrystallized grains with substantial grain boundary curvatures and long warm worked grains with no significant grain boundary curvatures. 


\section{3: X-RAY DIFERACTION}

Electrolytic extraction of the insolubles from the base metal was performed using a solution of $15 \% \mathrm{HCl}$ in methanol with a platinum cathode (according to the ASTM E 963 schedule, used for extraction of carbides, borides and topologically close packed phases in superalloys). After sufficient dissolution of the base metal, the residue was collected from the solution by centrifuging. X-ray diffraction studies were performed on bulk specimens as well as on the electrochemically extracted residue using $\mathrm{Cu}_{u} \mathrm{~K}_{\alpha}$ radiation with a nickel filter by diffractometry. The precision lattice parameter values were determined using the CELREF program [82], which employs a least square refinement of the unit cell dimensions.

\section{4: OPTICAL AND SCANNING ELECTRON MICROSCOPY}

All the microstructural conditions prior to electron beam welding were studied by optical microscopy, scanning electron microscopy and $x$-ray microanalysis (energy dispersive spectrometry (EDS)) to understand the nature and distribution of grains, precipitates and insolubles. Mean grain size under all conditions were determined either by using a Lietz image analyzer (fine grain sizes) or manually by using mean linear intercept method. The grain size in samples with warm worked grains was determined in two perpendicular directions, one along the long dimension of the warm worked grains and the other along the short dimension. SEM studies were undertaken both under secondary emission mode (SE) and under back scattered emission (BSE) mode. Metallographic preparation of samples were performed using standard techniques.

The bead on plate welds were sectioned transverse to the welding direction for optical, SEM and TEM examination. Metallographic sample preparation was again carried out using standard techniques. A variety of electropolishing solutions were used for electropolishing and/or electroetching in order to clearly delineate the feature of interest under study with clarity and without etching artefacts. For example, as would be shown in chapter 4 , the true morphology of the HAZ microfissures is lost, if the sample is etched or elec- 
tropolished with reagents that attack the solidification constituents, which in the case of Incoloy 903 are gamma and interdendritic MC carbides that form in the microfissures.

The fusion zone and heat affected zone microstructures were then studied by optical and scanning electron microscopy. Optical microscopy observation were carried out on both under normal illumination and under polarized illumination in the differential interference contrast (DIC) mode. A variety of SEM techniques viz., SEM/SE, SEM/BSE, SEM/EDS, SEM/line scanning and SEM/x-ray imaging were utilized to study the fusion zone and HAZ microstructures. All the SEM/EDS $x$-ray acquisition in this study were done with a JEOL-EDS system (silicon doped lithium high take off angle detector with a beryllium window) equipped with a Tracor Northern 5500 multichannel analyzer. All the SEM/EDS spectral quantification was undertaken using the 'MICROQ' program (supplied by Tracor Northern) which incorporates ZAF correction and deconvolution of the overlapped peaks from acquired spectral standards of elements of interest by using pure metal standards. The SEM/x-ray images were enhanced prior to photographing utilizing digital image processing with the image processing program (IPP) provided by Tracor Northern.

\section{5: ANALYTICAL ELECTRON MICROSCOPY}

Carbon extraction replica's were prepared by standard techniques from all the samples and examined in TEM for morphological, crystal structure and microanalytical studies of the precipitates. The lattice parameters of the phases observed in the base metal, fusion zone and HAZ were determined from numerous reflections obtained from the SADP's and microdiffraction patterns and refined with the CELREF program. Care was taken to include in the refinement only the averaged values of interplanar spacings obtained from low index reflections to minimize measurement errors. All interplanar spacing measurements were obtained directly from the SADP negatives. The camera length calibration was carried out using ring patterns obtained from thin films of evaporated high purity aluminum placed on a copper grid. Corrections due to specimen tilt were however, not compensated for. 
All the microanalyses were performed on EDS spectra obtained from thin precipitates on the carbon extraction replica on either carbon coated nylon grids or copper grids with the TEM operating with a tungsten filament at an accelerating voltage of $200 \mathrm{KV}$ in a focussed nanoprobe mode with a spot size of $40 \mathrm{~nm}$ (S mode i.e., with condenser minilens off and with alpha selector turned fully clockwise to provide a maximum convergence angle). A double tilt holder with zero tilt readings was utilized. The acquisitions and analyses were performed with a Tracor Northern high take off angle Si( Li) detector coupled to a Tracor Northern model TN 5400 multichannel analyzer. Semi-quantitative analysis using theoretical Cliff-Lorimer $k$ factors was utilized for the estimation of the chemical composition of the phases. The chemical analysis was performed using the standardless metallurgical thin film software package provided by Tracor Northern. The program uses Kramer's law for background modelling but does not perform any deconvolution routine for assessment of the relative contribution of elements to overlapped peaks. However, care was taken to ensure that the $X$-ray acquisition came from only very thin areas and also only from precipitates not aligned along strongly diffracting orientations. At least twenty-five precipitates of each kind observed in the alloy were analyzed to determine the average microanalytical information. 


\section{Chapter 4}

\section{RESULTS AND DISCUSSION}

\section{1: BASE METAL MICROSTRUCTURAL CHARACTERIZATION}

\subsection{1: Introduction}

It was emphasized in chapter 2 that the base metal microstructure prior to welding plays a major role in HAZ microfissuring and in determining the properties of weldments. Of particular interest are (i) matrix and grain boundary phases, their composition, their range of stability, their size and distribution and whether they lead to the formation of liquid films far below the bulk solidus temperature by eutectic reaction with the matrix (ii) the presence and/or segregation of tramp elements like boron, sulfur, phosphorus etc. on the grain boundaries (iii) whether the alloy is in a solution treated condition or in an aged condition and (iv) the grain size. Hence, it becomes imperative to characterize completely the starting microstructure prior to welding. This section describes the results of the microstructural characterization of thermo-mechanically processed Incoloy 903 given final solution treatments at different temperatures.

\subsection{2: Optical Microscopy}

The microstructure of the as received billet of Incoloy 903 prior to thermo-mechanical processing, as shown in fig 4.1(a), consisted of equiaxed grains with large insolubles randomly distributed in the material. The grain boundaries as observed by optical microscope and SEM were clean with no fine precipitates or insolubles on them. The microstructure of the as-forged alloy and the samples forged and solution treated at $840^{\circ} \mathrm{C} / \mathrm{I}$ h and $925^{\circ} \mathrm{C}$ / 
$1 \mathrm{~h}$ consisted of a bimodal distribution of grain sizes. Fig 4.1(b,c) show optical micrographs of the forged and solution treated ring and the as-forged alloy solution treated for an hour at $925^{\circ} \mathrm{C}$ respectively. Large warm worked grains and fine recrystallized grains are observed in the figures. The microstructure of the forged alloy solution treated at $950^{\circ} \mathrm{C}$ consisted of a large amount of small partially recrystallized grains with no clearcut warm worked grains, fig 4.1(d), while the microstructure of the forged alloy solution treated at $975^{\circ} \mathrm{C}$ consisted of fully recrystallized grains. The microstructure of the forged and solution treated ring further solution annealed at $1100^{\circ} \mathrm{C}$ consisted of equiaxed recrystallized grains.

A wide range of mixed grain structures, from fine recrystallized grains to coarse warm worked grains can be produced by careful control of the forging temperature, strain rate, total deformation and the final solution treatment temperature [83]. The optimum properties appear to be associated with the formation of the necklace structure, in which the material has only partially recrystallized $[73,77,78,83]$. Recrystallized grains should however, not be distributed randomly throughout the structure but should be in bands closely associated with the grain boundaries, fig $4.1(b, c)$. Thus each warm worked grain is surrounded by a necklace of small recrystallized grains. It has also been reported [83] that for a good combination of fatigue and creep resistance, the proportion of recrystallized to unrecrystallized grains should be within a certain range. The commercial solution treatment given to thermo-mechanically processed Incoloy 903 is generally in the range of $840^{\circ} \mathrm{C}-860^{\circ} \mathrm{C}$ to develop a controlled necklace structure.

In figs 4.1(b-d) large insolubles in the matrix, 5 to $10 \mu \mathrm{m}$ in size, generally aligned in the rolling direction and fine precipitates on the grain boundaries and prior grain boundaries can be observed. The thermo-mechanical processing the alloy has undergone is a multistep soaking, rolling and forging treatment. In this process, some of the warm worked grains formed in the earlier steps get recrystallized. However, the precipitates that existed on these annealed warm worked boundaries remain. This feature is illustrated in fig 4.1 (d) 


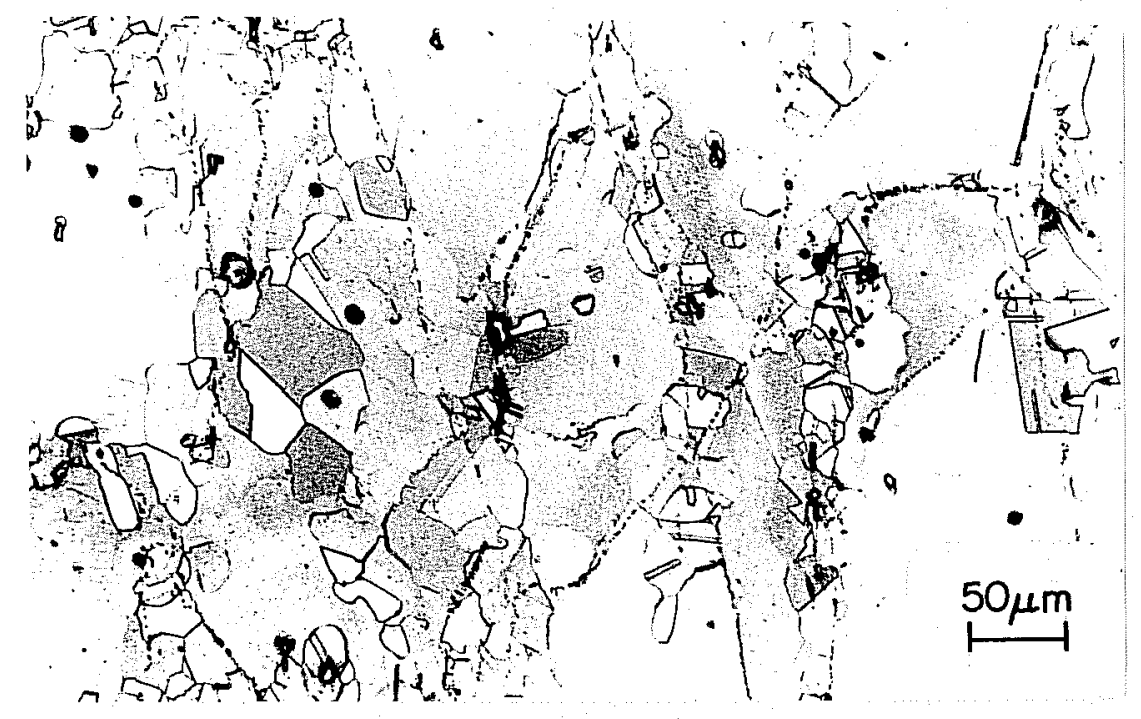

fig 4.1(c): Optical micrograph of the as-forged alloy 903 solution treated at $925^{\circ} \mathrm{C} / 1 \mathrm{~h}$.

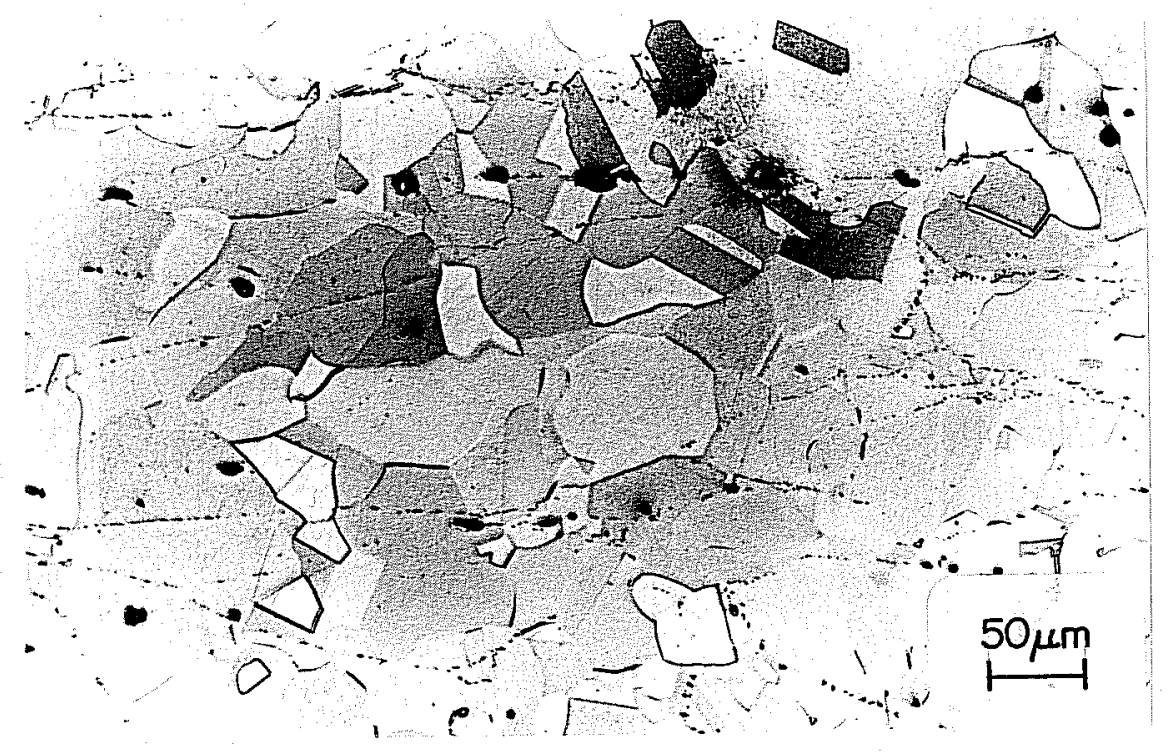

fig 4.1(d): Optical micrograph of as-forged alloy solution treated at $950^{\circ} \mathrm{C} / 1 \mathrm{~h}$. 
where the warm worked grains outlined by the fine precipitates have been annealed out. These boundaries are referred to as prior grain boundaries in subsequent discussion. As can be seen from the optical micrographs, the only difference between the two different forgings [as-forged as well as various forged and solution treated ones vis a vis forged and solution treated ring] is the existence of precipitates on the prior grain boundaries in one set of forgings. In the case of the forged and solution treated ring, all the precipitation during thermo-mechanical processing was confined to the existing boundaries, as can be observed from fig $4.1(\mathrm{~b})$. In the case of the forged alloy and alloy forged and solution treated at various temperatures fine precipitates were observed on existing grain boundaries as well as on prior grain boundaries. Precipitates on prior grain boundaries in alloy forged and solution treated at $925^{\circ} \mathrm{C} / 1 \mathrm{~h}, 950^{\circ} \mathrm{C} / 1 \mathrm{~h}$ and $975^{\circ} \mathrm{C} / 1 \mathrm{~h}$ were observed possibly because of the increased recrystallization in these cases as compared to the forged and solution treated ring. This may be due to the allowable uncertainity in soaking and warm working schedules in the industry leading to greater recrystallization in one of the cases.

In the forged and solution treated ring, the proportion of fine recrystallized grains to the large warm worked ones varied considerably from the inner edge of the ring to the outer edge. However, the proportion was constant along any given diameter of the ring. There existed areas where $95 \%$ of the grains were large warm worked ones. The proportion of the fine recrystallized grains was observed to increase as one moves towards either of the edges of the ring.

The warm worked grain boundaries were observed to be long and straight with no significant grain boundary curvature while the fine recrystallized grains outlining the large warm worked grains were small with substantial mean grain boundary curvature. The recrystallized grain boundaries in the samples solution treated at $975^{\circ} \mathrm{C}$ for one hour also had significant local curvatures owing to pinning by fine carbides. The recrystallized grain boundaries in samples solution treated at $1100^{\circ} \mathrm{C}$ were observed to be straight without significant grain boundary curvature. 
Table 4.1: Grain sizes of Incoloy 903.

$\begin{array}{lll}\text { Sample } & \begin{array}{l}\text { Long } \\ \text { Dimension }\end{array} & \begin{array}{l}\text { Short } \\ \text { Dimension }\end{array} \\ \text { Preforged: } & & \\ \text { Parallel Section } & & \\ \text { Perpendicular Section } & 23 \mu \mathrm{m}\end{array}$

Forged and Solution Treated Ring:

Parallel Section $\quad 447 \mu \mathrm{m} \quad 252 \mu \mathrm{m} \quad 23 \mu \mathrm{m}$

(to the radius)

Perpendicular

$339 \mu \mathrm{m}$

$22 \mu \mathrm{m}$

$69 \mu \mathrm{m}$

Section

(to the radius)

Forged:

Section 1

$410 \mu \mathrm{m}$

$200 \mu \mathrm{m}$

$46 \mu \mathrm{m}$

Section 2

$420 \mu \mathrm{m}$

$205 \mu \mathrm{m}$

$53 \mu \mathrm{m}$

Section 3

$520 \mu \mathrm{m}$

$200 \mu \mathrm{m}$

$28 \mu \mathrm{m}$

$925^{\circ} \mathrm{C} / 1 \mathrm{~h}:$

$385 \mu \mathrm{m}$

$185 \mu \mathrm{m}$

$30 \mu \mathrm{m}$

$950^{\circ} \mathrm{C} / \mathrm{Ih}$ :

$42 \mu \mathrm{m}$

$975^{\circ} \mathrm{C} / 1 \mathrm{~h}:$

$65 \mu \mathrm{m}$ 
Table 4.1 (Contd)

$\begin{array}{llll}\text { Sample } & \text { Long } & \text { Short } & \text { Recrystallized } \\ & \text { Dimension } & \text { Dimension } & \end{array}$

$1100^{\circ} \mathrm{C} / 1 / 2 \mathrm{~h}$

$95.2 \mu \mathrm{m}$

$1100^{\circ} \mathrm{C} / \mathrm{hh}$

$120 \mu \mathrm{m}$

$1100^{\circ} \mathrm{C} / 2 \mathrm{~h}$

$150 \mu \mathrm{m}$

$1100^{\circ} \mathrm{C} / 4 \mathrm{~h}$

$192 \mu \mathrm{m}$

$1100^{\circ} \mathrm{C} / 8 \mathrm{~h}$

$220 \mu \mathrm{m}$

Note: The samples given solution treatments at $1100^{\circ} \mathrm{C}$ were from the forged and solution treated ring. 
The grain sizes of all the microstructural conditions was estimated by mean linear intercept method and the results are presented in Table 4.1. Since, the warm worked grains are textured with consistent long and short dimensions, the grain size in both orientations were seperately determined and are reported in Table 4.1.

\subsection{3: X-ray Diffraction and Scanning Electron Microscopy}

$X$-ray diffraction studies were performed on the insolubles electrolytically extracted from the bulk material after the thermo-mechanical processing. The major constituent was observed to have an fcc crystal structure with a lattice parameter of $4.434 \AA$. Minor peaks were also observed and were indexed as fcc with a lattice parameter of $4.25 \AA$. Comparison of the data with the available literature [84-88] indicates the major component of the insolubles to be carbides and/or carbonitrides ( $M X$ type, fcc, $X=C / N / C, N$ and $\mathrm{M}=\mathrm{Ti}$ and/or $\mathrm{Nb}$ in the present case) based on titanium and niobium. The minor component appears to be TiN. It should be mentioned that very fine powder floated away during centrifuging and during the slide preparation for diffractometry.

Fig 4.2 shows a SEM/BSE image of a large insoluble. Two different phases in the insoluble, an outer region rich in higher atomic number elements than the matrix and an inner region (hereafter referred to as the nucleus) with lower atomic number elements than the matrix, can be identified. The chemical composition of these regions as determined by SEM/EDS quantitative microanalyses (table 4.2), indicates the inner darker phase to be based on titanium and the larger outer phase to be based on niobium. The experimental techniques utilized in the present investigation provide no reliable evidence as to the proportion of carbon and nitrogen in any of the $M X$ precipitates. The lattice parameters of the various carbides and carbonitrides based on niobium and titanium are too similar to one another [84-88] to allow any firm conclusion to be made based on the lattice parameter data. Hence, in further discussion, all of them are referred to as carbides. The metallic composition (table 4.2) of the outer insoluble of the coarse carbides is 79.3 at 
$\% \mathrm{Nb}$ and 20.7 at $\% \mathrm{Ti}$ and that of the inner nucleus is 7.4 at $\% \mathrm{Nb}$ and 92.6 at \% Ti. These values conform well with prior reported data on carbides in similar families of superalloys $[77,78,89]$.

The SEM/EDS microanalyses confirm the existence of two different kinds of distinct phases in the large insoluble. Hence, $x$-ray studies should yield two different lattice parameters. As discussed earlier, $x$-ray diffraction results on extracted insolubles did indicate two distinct carbides with lattice parameters of $4.434 \AA$ and $4.25 \AA$. The reported values for lattice parameters of TiN and TiC are $4.244 \AA$ and $4.33 \AA$ respectively [85]. Hence, in conjunction with the microanalytical data, it can be concluded that the inner nucleus is $\operatorname{TiN} / \mathrm{Ti}(\mathrm{C}, N)$. The preferred order of formation of $M C$ carbides in superalloys is $\mathrm{NbC}$ and then $\mathrm{TiC}$ in order of decreasing stability [90]. This order is not the same as dictated by thermodynamics, which is $\mathrm{TiC}$ and then $\mathrm{NbC}[90]$. Carbides and nitrides are believed to form in superalloys before freezing [90]. During solidification, TiN/Ti(C,N) is the first phase to form from the melt. These particles serve as sites for the nucleation and growth of primary carbides from the melt. As discussed above, from kinetic considerations, the formation of $\mathrm{NbC}$ is favored over the formation of $\mathrm{TiC}$. Hence, $\mathrm{NbC}$ nucleates and grows around $\operatorname{TiN} / \operatorname{Ti}(C, N)$. This yields the experimentally observed duplex insoluble which has an inner nucleus comprised of TiN/Ti(C,N) and an outer crust made up of Nb-rich carbide.

Fig 4.3 shows a SEM/BSE image of the as-forged alloy. Fine precipitates decorating grain boundaries and prior grain boundaries can be observed. Their chemical analysis as obtained by SEM/EDS is not reported because the electron probe size of SEM restricted their precise determination.

\subsection{4: Analytical Electron Microscopy}

Fig 4.4(a,b) shows a TEM bright field image from a carbon extraction replica of an as-forged alloy. Fine irregular precipitates along boundaries and prior grain boundaries can be observed. Fig 4.4 (c) shows the microstructure of an individual particle. It can be 


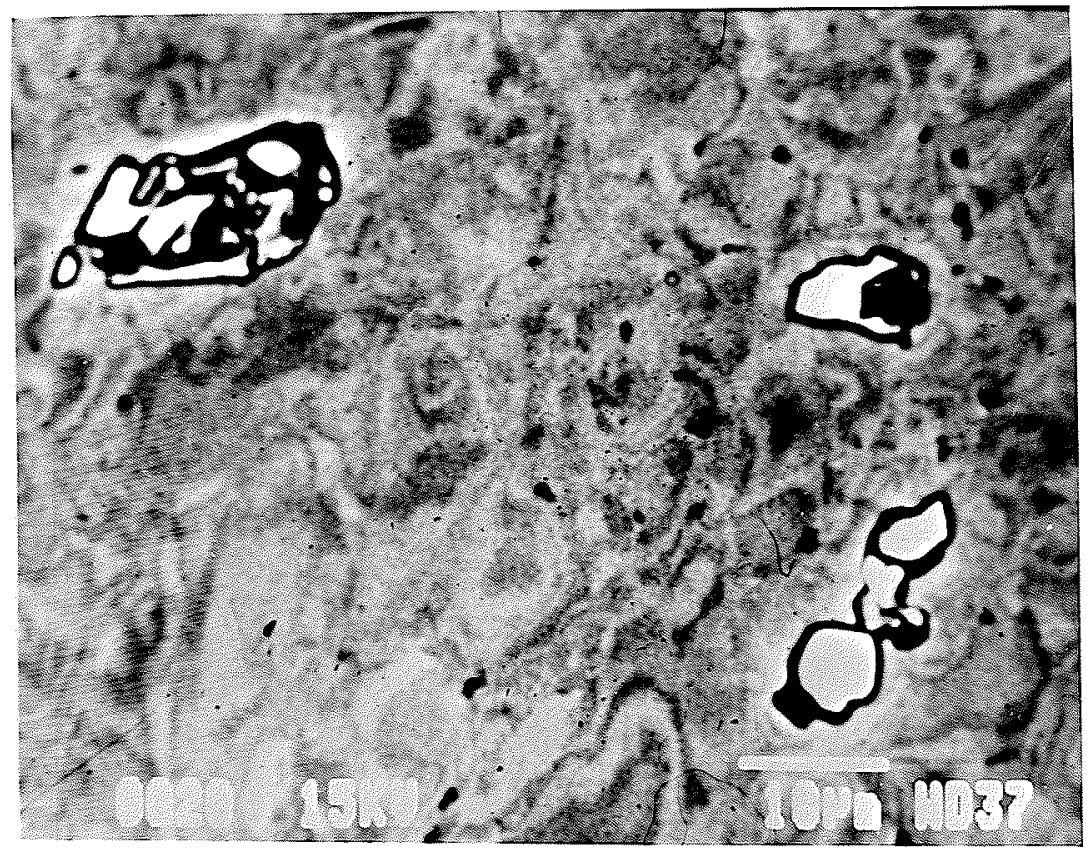

fig 4.2: SEM/BSE image of a large insoluble in the forged and solution treated ring.

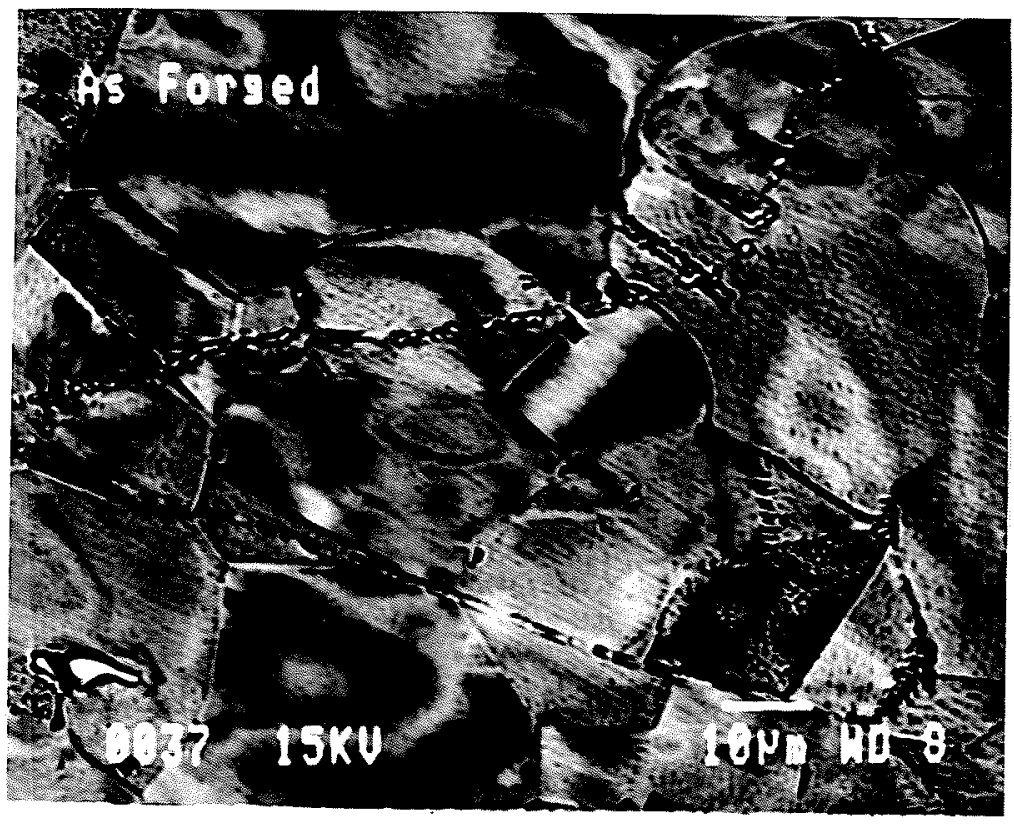

fig 4.3: SEM/BSE image showing fine precipitates on the grain boundaries and prior grain boundaries in an as-forged alloy. 
seen that each particle is composed of fine misoriented crystallites with internal defects within the crystallites. Fig 4.4(d-f) shows the SADP's obtained from these crystallites in three crystallographic orientations. Analysis of the various diffraction patterns indicate them to belong to an orthorhombic crystal structure with cell dimensions of a $=5.99 \AA$; $\mathrm{b}=3.35 \AA$; and $\mathrm{c}=7.06 \AA$. Fig $4.4(\mathrm{~g})$ shows a TEM/EDS spectrum obtained from one of these particles. A phosphorus $K_{\alpha}$ peak can be observed to the left of the niobium $L$ multiplet. Though there is a partial overlap of the phosphorus $\mathrm{K}$ doublet with the niobium $\mathrm{L}$ multiplet, the clearcut bulge to the left of $\mathrm{Nb}$ peak is a positive identification for the presence of phosphorus in the precipitate. Another kind of regular fine cuboidal precipitates was also observed on the boundaries and these precipitates were found to be $M X$ carbides (tables 4.2 and 4.3 ,figs $4.5,4.6$ ) by electron diffraction and TEM/EDS microanalytical studies. For comparison, an EDS spectrum from a fine $\mathrm{MX}$ carbide found in the as-forged alloy solution treated at $975{ }^{\circ} \mathrm{C}$ for one hour is shown in fig 4.5. In this case, one can observe a pure Niobium L multiplet.

The chemical analysis as obtained from TEM/EDS of the irregular precipitate particles observed in various samples is given in Table 4.2. Since, all the phases studied in the present investigation were observed to be non-stoichiometric, the chemical analysis is presented as (i) the average value (ii) the maximum value and (iii) the minimum value for each element in a given type of precipitate. Furthermore, since, only a partial overlap existed between the niobium $L$ multiplet and the phosphorus $K$ doublet, the phosphorus compositions were approximately assessed. Despite the uncertainty in the phosphorus and niobium composition analyses because of peak overlap, both are being reported in Table 4.2 to give an indication of the composition values to a first approximation. The total possible error due to the semi-quantitative analysis is assessed to be in the range of $8 \%$ to $13 \%$. An alternate approach [89] would be to ignore the exclusive phosphorus counts in the spectra and perform the analyses. This amounts to normalizing the rest of the composition values. Both methods are prone to error because in the latter approach the 
total niobium counts are overestimated by the amount of buried phosphorus counts and the composition of the rest of the metallic elements also get overestimated because of the elimination of the exclusive phosphorus count. This approach [89] does not provide any idea of the phosphorus composition. The ideal solution would have been to perform deconvolution of the overlapped peaks but in the absence of this technique it was felt that reporting the phosphorus composition would be helpful in fully appreciating the nonstoichiometric nature of the phase under investigation. The appreciable differences in the composition data in Table 4.2 are significant despite the uncertainity introduced in the spectral analyses.

Comparison of the crystal structure and the composition data from the irregular precipitates with available literature $[89,91]$ indicates them to belong to an MNP-type phosphide ( $M$ and $N$ are different kind of metal atoms $M=N b, T i ; N=N i, C o, F e$ ) which till to date has been reported only once in superalloys [89].Vincent's data [89] on MNP type phosphides observed in the heat affected zone (HAZ) of alloy 718 weldments is given in table 4.3 for comparison. Vincent [89] has reported only the composition analysis of the metallic elements in the phosphides. Vincent [89] noted that the phosphorus composition appeared low for an equi-atomic MNP-type phosphide. The main evidence for the existence of MNP-type phosphides stemmed from the crystal structure, lattice parameter and the composition of the $M$ and $N$ type atoms.

Unlike Vincent [89] who found only twenty particles in one of the Inconel 718 weldments, in the present investigation, MNP-type phosphides were found extensively in the as-forged alloy 903 and samples forged and solution treated at $840^{\circ} \mathrm{C}$, and $925^{\circ} \mathrm{C}$. Some of the phosphides, except in the $840^{\circ} \mathrm{C} / 1 \mathrm{~h}$ solution treated ring, were found outlining the prior grain boundaries while in the ring forged and solution treated at $840^{\circ} \mathrm{C} / 1 \mathrm{~h}$ both phosphides and carbides were found only on the grain boundaries. The alloy solution treated at $975^{\circ} \mathrm{C}$ for one hour had very few phosphides (less than $5 \%$ of the total particles, the rest being carbides). These phosphides were small and are likely to be the 

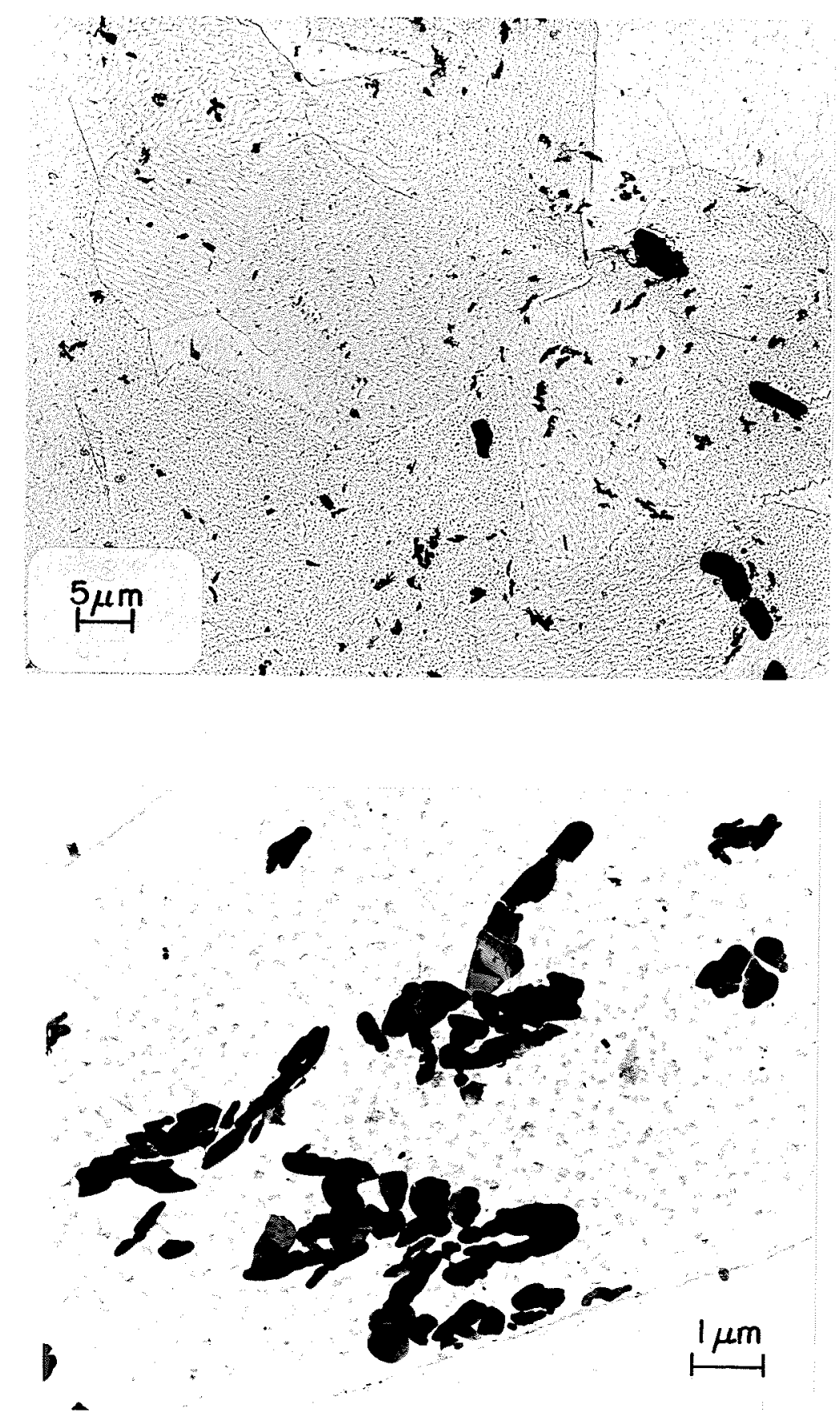

fig 4.4(a,b): A TEM, bright field image of fine irregular precipitates on carbon extraction replica from an as-forged alloy. 


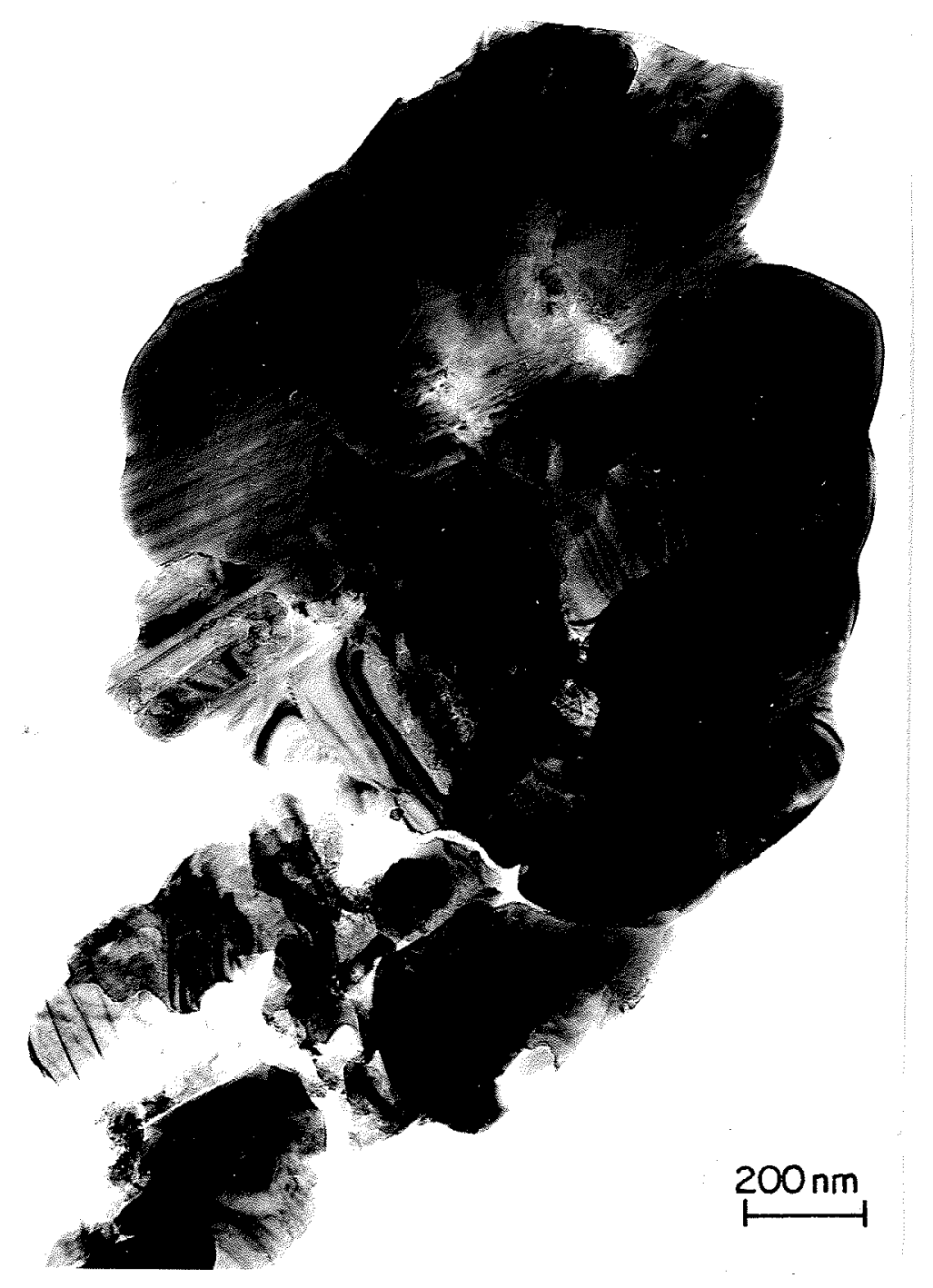

fig 4.4(c): A TEM, bright field image of a fine irregular precipitate on carbon extraction replica from an as-forged alloy. 


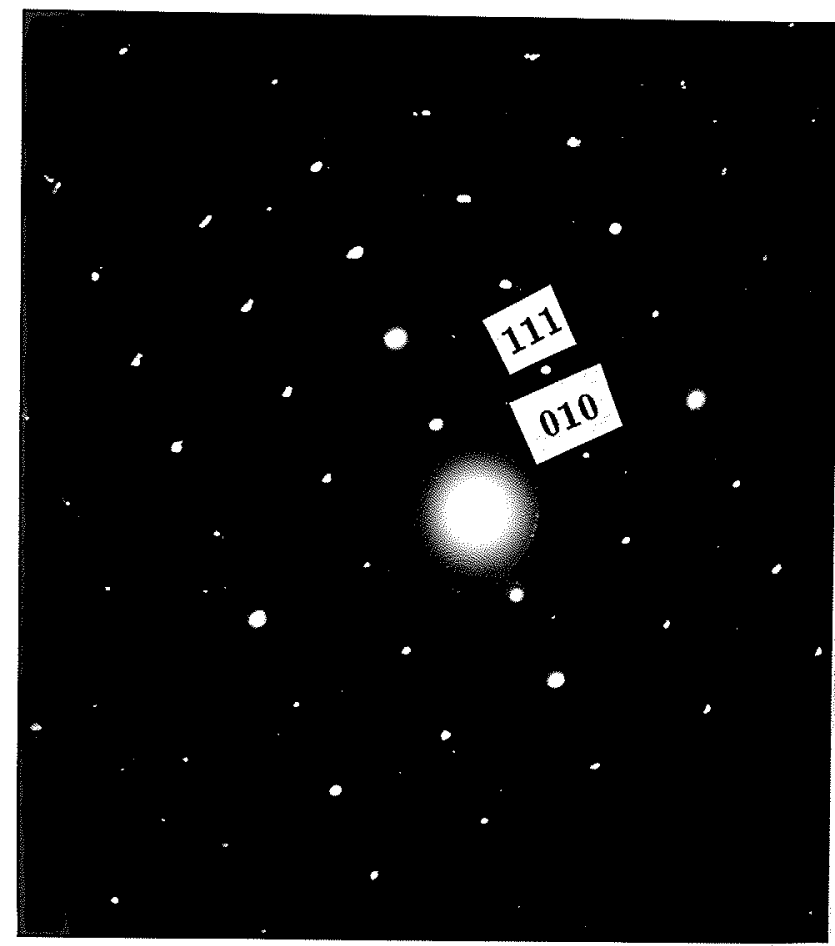

fig 4.4(d): SADP from a phosphide, $Z=[10 \overline{1}]$.

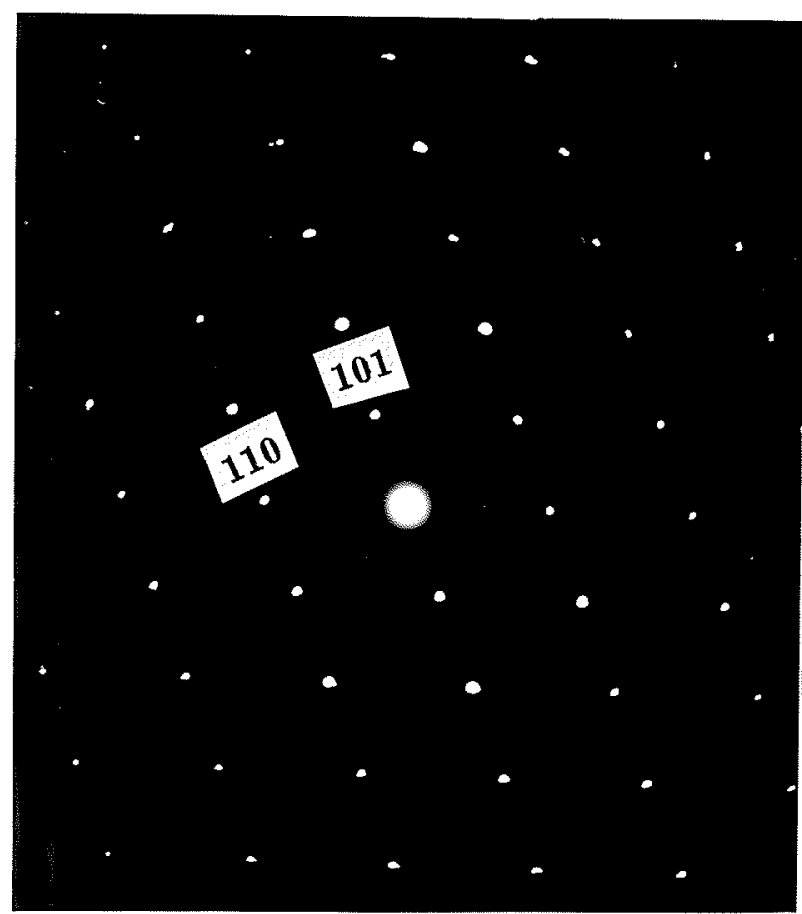

fig 4.4(e): SADP from a phosphide, $Z=[\overline{1} 11]$. 


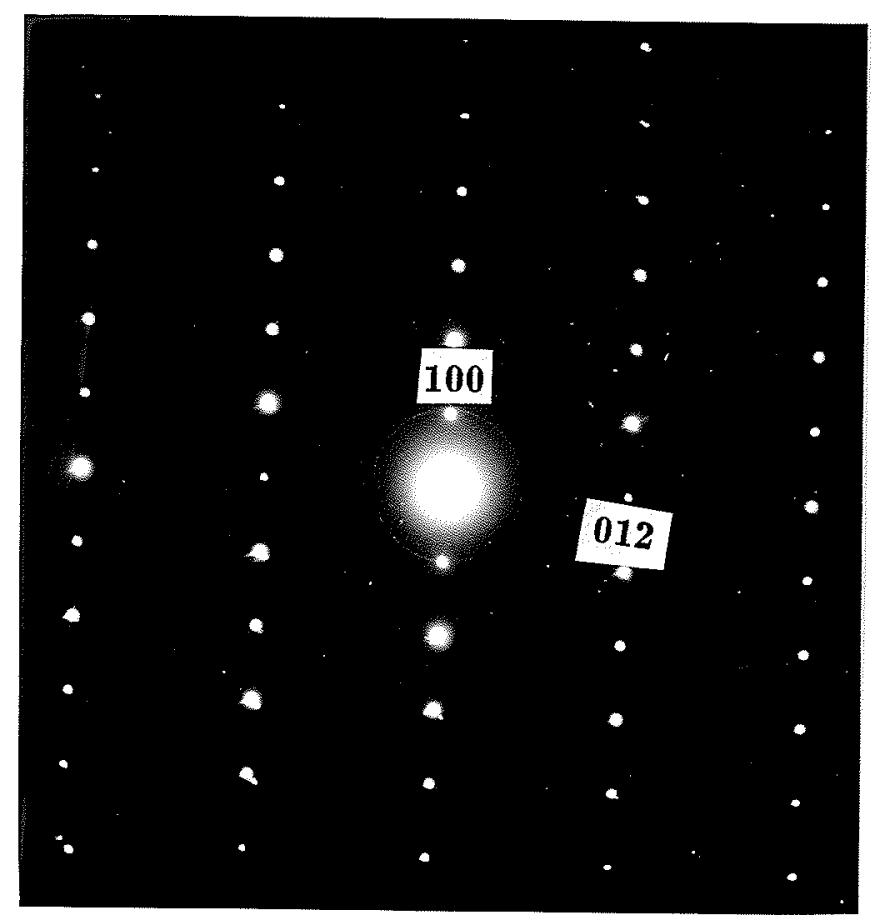

fig 4.4(f): SADP from a phosphide, $Z=[02 \overline{1}]$.

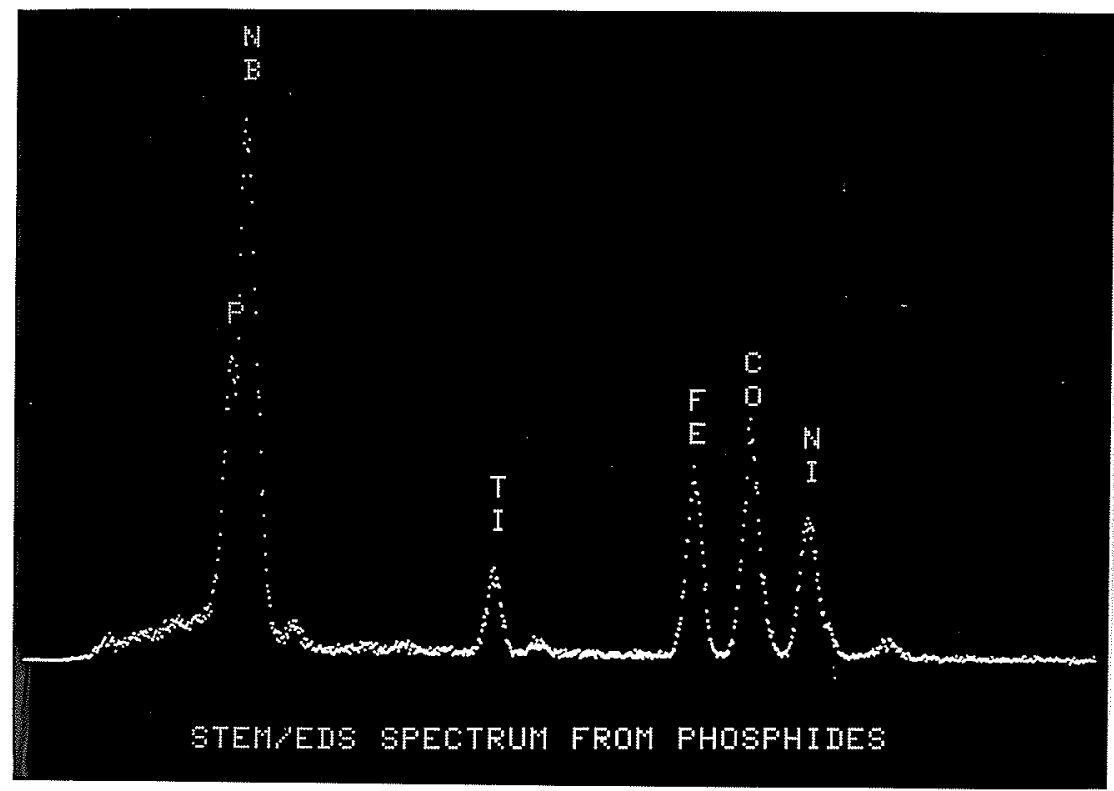

fig 4.4(g): A typical STEM/EDS spectrum obtained from particles in fig 4(a-c). 


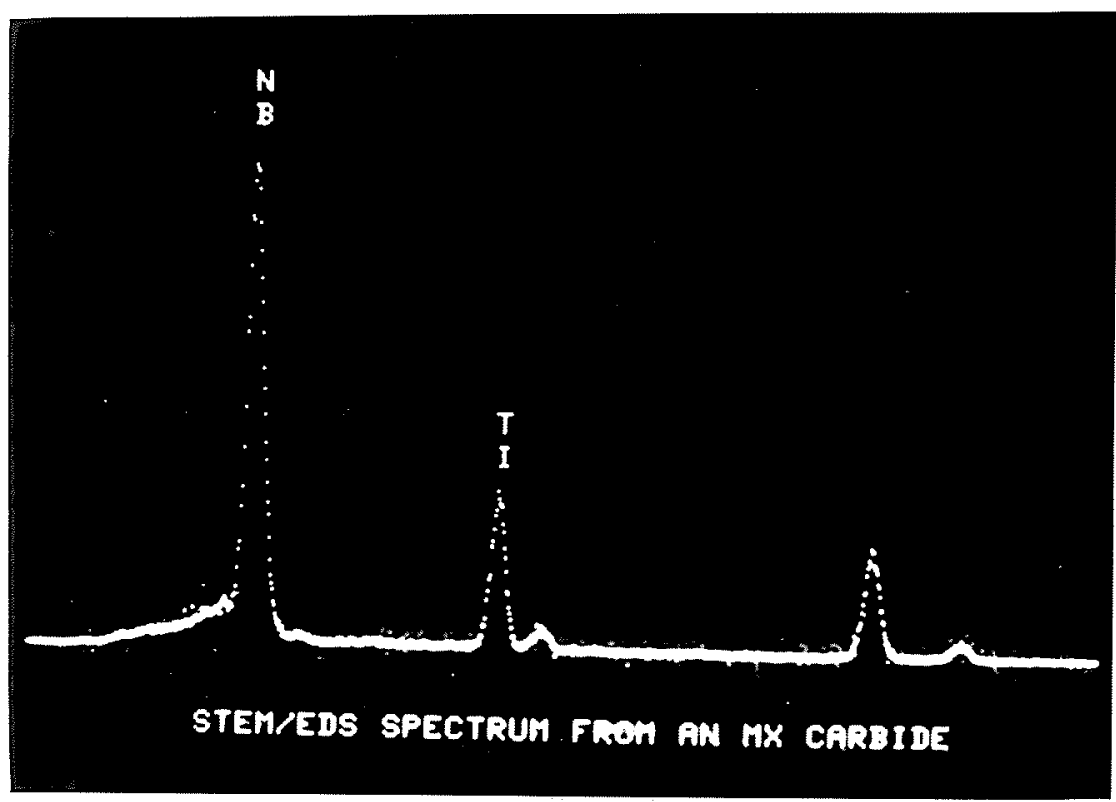

fig 4.5. A typical STEM/EDS spectrum obtained from a Niobium rich MX type carbide.

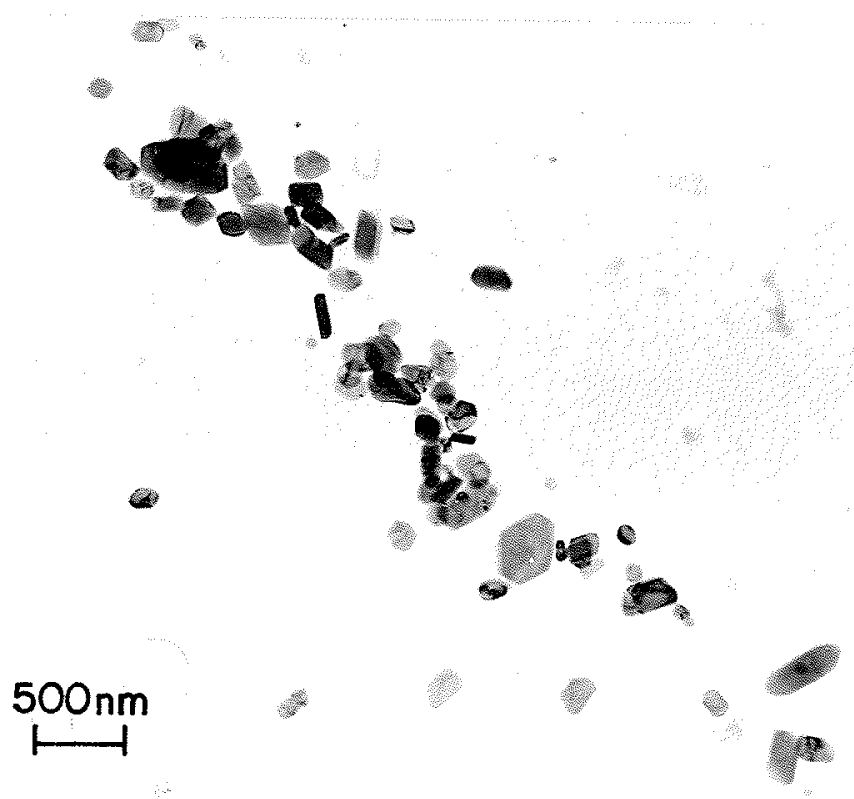

fig 4.6: A TEM, bright field image of fine carbides on carbon extraction replica from an as-forged alloy solution treated at $975^{\circ} \mathrm{C} / 1 \mathrm{~h}$. 
Table 4.2: Composition of insolubles and precipitates

1.Coarse Carbides, MX type
(a) Inner Phase
$\mathrm{Nb} \quad \mathrm{Ti}$
$\begin{array}{lll}\text { Average } & 7.4 & 92.6\end{array}$
$\begin{array}{lll}\text { Maximum } & 9.3 \quad 95.6\end{array}$
$\begin{array}{lll}\text { Minimum } & 4.4 & 90.7\end{array}$

(b) Outer Phase

$\begin{array}{ccc}\text { Average } & 79.3 & 20.7 \\ \text { Maximum } & 84.1 & 24.0 \\ \text { Minimum } & 76.0 & 15.9\end{array}$

\section{Fine Carbides,MX type}

(a) Niobium Rich

$\mathrm{Nb} \quad \mathrm{Ti}$

$\begin{array}{crr}\text { Average } & 79.5 & 20.5 \\ \text { Maximum } & 85.8 & 22.8 \\ \text { Minimum } & 77.9 & 14.0\end{array}$

(b) Titanium Rich

$\begin{array}{ccc}\text { Average } & 57.8 & 42.2 \\ \text { Maximum } & 67.6 & 57.1 \\ \text { Minimum } & 39.2 & 32.4\end{array}$


Table 4.2 (contd)

3.MNP type Phosphides
(a) As Forged
$\mathrm{Nb} \quad \mathrm{Ti} \quad \mathrm{Fe}$
Average
44.5
2.5
13.0
Co
$\mathrm{Ni} \quad \mathrm{P}$
$\mathrm{Cr}_{\mathrm{r}}$ Mo
Maximum
$\begin{array}{ll}46.7 & 3.7\end{array}$
21.1
16.1
$11.7 \quad 11.7$
Minimum
41.6
1.8
10.2
16.9
$13.5 \quad 18.3$

(b) Forged and S.T. $975^{\circ} \mathrm{C}$

$\begin{array}{cllllll}\text { Average } & 37.1 & 4.5 & 11.7 & 15.8 & 11.1 & 20.2 \\ \text { Maximum } & 40.3 & 7.6 & 16.8 & 22.4 & 15.8 & 24.9 \\ \text { Minimum } & 30.1 & 2.9 & 9.7 & 13.3 & 9.2 & 11.6\end{array}$

(c) Inconel 718 welds

$$
\text { (ref 8) }
$$

$45.0 \quad 2.0$

$7.0-$

$28.0-$

14.0

4.0

Occupancy ratio

$\mathrm{Co}: \mathrm{Fe}: \mathrm{Ni}$

(MNP phosphides)

(a) As Forged

$\begin{array}{lll}0.39 & 0.32 & 0.29\end{array}$

(b) Forged and S.T. $975^{\circ} \mathrm{C}$

$\begin{array}{lll}0.41 & 0.30 & 0.29\end{array}$

Note: (i) All compositions are given in at \%. (ii) All the compositions except for coarse carbides have been determined by STEM/EDS from particles on carbon extraction replica. The data for coarse carbides have been obtained using SEM/EDS.(iii) All carbides found in alloy 903 were of $M X$ type, where $M$ refers to the metallic component ( $N b, T i$ in the present case) and $\mathrm{X}$ is $\mathrm{C}$ or $\mathrm{N}$ or $(\mathrm{C}, \mathrm{N})$. Only the metallic compositions are provided in the table. (iv) In MNP Phosphides M refers to the $4 d / 5 d$ transition metal atoms (Nb,Mo, Ta, Zr....) and $\mathrm{N}$ refers to the $3 \mathrm{~d}$ transition metal atoms $\left(\mathrm{Fe}, \mathrm{Co}, \mathrm{Ni}, \mathrm{Cr}_{1}, \ldots ..\right)$. (v) See text for the size,location and amount of various precipitates. 
Table 4.3: Crystallographic data on precipitates and insolubles

\begin{tabular}{|c|c|c|c|c|c|}
\hline Precipitates & Technique & Space Group & \multicolumn{3}{|c|}{ Unit cell dimensions $(\AA)$} \\
\hline \multirow[t]{2}{*}{ Coarse Carbides } & $x$-ray & $\mathrm{Fm} 3 \mathrm{~m}$ & a 4.434 & $(v=87$ & 7.2) \\
\hline & $x$-ray & & a 4.25 & $(76.8)$ & \\
\hline Fine Carbides & SADP & $\mathrm{Fm} 3 \mathrm{~m}$ & a 4.431 & $(87)$ & \\
\hline ref $(89)$ & SADP & & a 4.42 & $(86.4)$ & \\
\hline $\operatorname{ref}(89)$ & SADP & & a 4.25 & $(76.8)$ & \\
\hline MNP Phosphides & SADP & Pnma & a 5.987 & & \\
\hline \multirow[t]{2}{*}{$(\mathrm{NbCoP})$} & & & b 3.349 & $(141.5)$ & $(0.848)$ \\
\hline & & & c 7.057 & & \\
\hline NBNiP & SADP & & a 6.111 & & \\
\hline \multirow[t]{2}{*}{ (ref 89) } & & & b 3.5 & $(151.64)$ & $(0.862)$ \\
\hline & & & c 7.09 & & \\
\hline $\mathrm{NbCoP}$ & x-ray & & a 6.112 & & \\
\hline \multirow[t]{2}{*}{ (ref 91,92$)$} & & & b 3.587 & $(153)$ & $(0.876)$ \\
\hline & & & c 6.978 & & \\
\hline $\mathrm{NbNiP}$ & $x$-ray & & a 6.108 & & \\
\hline \multirow[t]{2}{*}{ (ref 91,92) } & & & b 3.578 & $(155) \quad($ & $(0.861)$ \\
\hline & & & c 7.091 & & \\
\hline $\mathrm{NbFeP}$ & $x$-ray & & a 6.139 & & \\
\hline \multirow[t]{2}{*}{ (ref 91) } & & & b 3.585 & $(154.2)$ & $(0.876)$ \\
\hline & & & c 7.006 & & \\
\hline
\end{tabular}

Note: The unit cell volume $v\left(\AA^{3}\right)$ is given in the fist brace after the cell dimensions and the $a / c$ ratio is provided in the next brace. 
larger ones that have undergone incomplete dissolution. Their compositions have been analyzed and reported seperately in table 4.2. In the forged alloy solution treated ring (solution treatment in the range $840^{\circ} \mathrm{C}-860^{\circ} \mathrm{C}$ after the final thermo-mechanical pass) equal proportion of carbides and phosphides were observed while in the as-forged alloy, the proportion of phosphides to carbides was approximately $9: 1$.

As can be observed from the figs 4.4(a,b), the size of phosphides range from 0.1 to 0.5 $\mu \mathrm{m}$. These are very easily recognizable on the extraction replicas owing to their irregular shape, small misoriented crystallites and the presence of internal defects. As stated, the only other particles observed on the replicas were fine $M X$ type carbides which were also easily differentiable due to the distinct cuboidal shape, fig 4.6, and the absence of internal defects. In some instances, the carbides were found to precipitate alongside the phosphides. It should also be mentioned that in samples solution treated at $975^{\circ} \mathrm{C} / 1 \mathrm{~h}$, traces of a randomly distributed non-stoichiometric phase rich in $\mathrm{Fe}, \mathrm{Cr}$ and $\mathrm{Al}$ were also observed. The uniqueness of the phosphide phase, its non-stoichiometric nature, its presence on the grain boundaries, it being stable only below $975^{\circ} \mathrm{C}$ and the high niobium content requires a detailed discussion.

Many ternary equi-atomic phosphides, MNP, are ordered versions of the $\mathrm{CO}_{2} \mathrm{P}$ form of the anti- $\mathrm{PbCl}_{2}$ crystal structure. A large number of ternary silicides, germanides and phosphides crystallize with the $\mathrm{CO}_{2} \mathrm{P}$-type structure which is a subclass of the anti- $\mathrm{PbCl}_{2}$ type crystal structure. The anti- $\mathrm{PbCl}_{2}$-structure has two crystallographically nonequivalent metal positions, $\mathrm{M}$ and $\mathrm{N}$. Compounds crystallizing with the anti- $\mathrm{PbCl}_{2}$-type-structure can be divided into two sub-classes with respect to their atomic coordination. Both sub-classes belong to the space group $P n m a$. Typical representatives for the two sub-classes are $\mathrm{CO}_{2} \mathrm{P}$ and $\mathrm{CO}_{2} \mathrm{Si}$. The differences in coordination between the two sub-classes are connected with the differences in the positional parameters of the atoms and also with an appreciable difference in the shape of the unit cells.It is also possible, barring borderline cases, to classify an anti- $\mathrm{PbCl}_{2}$-type compound with respect to its type of coordination merely by 
determining its a/c ratio, which lies in the range $0.67-0.73$ for $\mathrm{CO}_{2} \mathrm{Si}$ sub-class and in the range $0.79-0.85$ for the $\mathrm{Co}_{2} \mathrm{P}$ subclass [91].

Atomic coordination values $[91,92]$ show that a structure of the $\mathrm{CO}_{2} \mathrm{P}$ subclass would be particularly suited for ternary compounds containing two kinds of metal atoms with different radii, the larger metal atoms occupying the $M$ positions with the highest coordination number and the smaller metal atoms occupying the $N$ positions. The non-metal atoms have the coordination number nine, which is particularly common with phosphides and arsenides and is frequently found in germanides. These compounds also known as $E$ phases $[93,94]$, contain $4 \mathrm{~d}$ or $5 \mathrm{~d}$ transition metal atoms as the larger atoms and $3 \mathrm{~d}$ transition metal atoms as the smaller atoms. A number of MNP systems exist and table 4.3 lists the ones relevent to the present investigation along with their unit cell dimensions. The abundance of $E$ phases is attributed to the particularly favorable geometrical properties of the crystal structure, allowing atoms of different radii to be accommodated within a comparatively small unit cell $[91,95]$.

Rundquist and Nawapong [91] had made an exploratory survey of M-N-P systems with $\mathrm{CO}_{2} \mathrm{P}$-type phases. Although they did not analyze in detail the phase relationships in these systems, they do report finding indications of extended homogeneity ranges of $\mathrm{CO}_{2} \mathrm{P}$-type phases in some of the systems. Deviations from ideal composition may occur through $\mathrm{M}-\mathrm{N}$ substitution and through formation of vacancies in the lattice $[91,96]$. Furthermore, the unit cell dimensions may exhibit variations even if the composition of the alloy is unchanged, depending on the degree to which $M$ and $N$ take ordered positions on the two nonequivalent metal atom sites in the structure [91]. Another interesting observation [97] for the $\mathrm{C}_{2} \mathrm{P}$-type phases with the general formula MNX ( $\mathrm{M}$ is a $4 d$ or $5 d$ transition metal, $N$ is a $3 d$ transition metal and $X$ is phosphorous, arsenic, silicon or germanium) is that MNX phases with cobalt as $N$ type invariably have the smallest unit cell volume for any fixed combination of $M$ and $X$ components even though the normal metal radius for $\mathrm{Ni}$ is smaller than Co. The major difference between NbCoP and NbNiP lies in the Co-Co 
and $\mathrm{Ni}-\mathrm{Ni}$ interatomic distances. The latter distances exceed the former by more than three times the difference between any other pair of corresponding average interatomic distance.

Comparison of the present data with those in the literature shows that the closest approach to the composition and the lattice constants of the phase under investigation is the NbCoP phase (Table 4.2,4.3) with $\mathrm{Ni}$ and Fe substituting for some of the $\mathrm{N}$ sites occupied by $C o$. The unit cell parameters agrees within an error of $2 \%$ for a, less than 7 $\%$ for $b$ and $1 \%$ for $c$ with NbCoP, within an error of $2 \%$ for $a$, less than $7 \%$ for $b$, and less than $1 \%$ for $c$ with NbNiP and within an error of less than $3 \%$ for a, less than $7 \%$ for $b$ and less than $1 \%$ for $c$ with NbFeP. It is, therefore, not possible to determine the identity of the phase from the lattice parameter data alone, as the data agrees within the same error with all the three related compounds, NbCoP, NbNiP and NbFeP. However, the microanalytical data provides some basis for the identification. The chemical analysis data obtained from the few, small, MNP type phosphides observed in the specimen solution treated at $975^{\circ} \mathrm{C}$ for one hour lends further credence to the fact that the observed phase is primarily based on NbCoP. The ratio of the amount of various elements in the MNP-type phosphides to that in the matrix ( i.e $k=$ at $\%$ in the phosphide/at $\%$ in the matrix) was observed to be 1.11 for $\mathrm{Co}$ and 0.31 for $\mathrm{Fe}$ as well as $\mathrm{Ni}$ in the as-forged specimen, while in the specimen forged and solution treated at $975^{\circ} \mathrm{C}$ the ratio was observed to be 1.09 for $\mathrm{Co}, 0.28$ for $\mathrm{Fe}$ and 0.29 for Ni. The occupancy ratio of $\mathrm{N}$ sites between $\mathrm{Co}, \mathrm{Fe}$ and $\mathrm{Ni}$ given in the last column of table 4.2 also shows that $\mathrm{CO}_{0}$ is the most preferred of the three. The amount of metallic elements observed to be present in the present study is consistent with equal proportions of $M(N b)$ and $N$ type ( $\mathrm{Co}, \mathrm{Fe}, \mathrm{Ni}$ ) atoms. These observations suggest that the phosphide observed in this investigation is based on NbCoP.

The composition of the nonmetallic component, i.e of phosphorus, appears low for an equi-atomic MNP-type phosphide. Two possible causes for this behavior are extended homogeneity range and/or phosphorus/boron substitution in the phosphide. The unam- 
biguous low phosphorus peak observed by Vincent [98] and in the present investigation on MNP-type phosphide could arise due to the presence of an extended homogeneity range. This view is supported by the non-stoichiometric nature of the phosphides analyzed in all the conditions of the alloy and by the observed decrease in niobium levels with a corresponding increase in phosphorus levels in the few phosphides observed in the as-forged alloy solution treated for an hour at $975^{\circ} \mathrm{C}$.

The metal rich transition borides, silicides and phosphides are known to have many common crystal chemistry properties [99]. Studies by Rundqvist [99] show noteworthy phosphorus/boron substitution in binary phosphide structures. Jeitscho [100] showed that some metal rich borides (MoCoB, WCoB and WFeB) crystallize with the anti- $\mathrm{PbCl}_{2}$ type structure like the $\mathrm{E}$ phase. The $\mathrm{NbCoB}$ structure was shown [101] to be a combination of the anti- $\mathrm{PbCl}_{2}$ type structure and $\mathrm{FeP}_{2}$-type structure. $\mathrm{NbFeB}$ is isostructural with $\mathrm{Fe}_{2} \mathrm{P}$ [102]. In all the above mentioned cases, boron has a coordination number of nine. Hence, one can see that some of the nonmetal sites in the MNX-type structures can be occupied by boron without any changes in the crystal structure. Extending these observations to MNP phosphides found in the present investigation, it can be assumed that some boron substitution for phosphorus can occur. The unit cell volume is much smaller with boron substitution. Some evidence for possible boron substitution is provided by the lower unit cell volume observed in this investigation as opposed to the values listed in the literature $[91,92]$. It should however, be pointed out that further confirmation is required through a detailed analysis of the phase relationships in the M-N-P systems of interest. Either similar studies on M-N-P-B systems or STEM/EELS studies on the phosphides are required to ascertain if any boron is present in MNP phosphide observed in Incoloy 903.

As can be seen from table 4.2 , the $\mathrm{Co}, \mathrm{Fe}$ and $\mathrm{Ni}$ content in the phosphides vary within a certain range from one precipitate to the other. This is due to the difference in the occupancy ratio of $\mathrm{N}$ type metal atom sites between $\mathrm{Co}, \mathrm{Fe}$ and $\mathrm{Ni}$. Depending on the relative amount of occupancy by $\mathrm{Fe}, \mathrm{Ni}$ and $\mathrm{Co}$ slight changes in the unit cell dimensions 
may be expected but the differences were too small to be detected by SADP's. No change in the lattice parameter values was observed for phosphides observed in the samples with different heat treatments.

As the solution treatment temperature after the final thermo-mechanical treatment is increased, increased precipitation of fine carbides on the grain boundaries was observed. $X$-ray diffraction on bulk samples solution treated at $925^{\circ} \mathrm{C} / 1 \mathrm{~h}, 950^{\circ} \mathrm{C} / 1 \mathrm{~h}$ and $975^{\circ} \mathrm{C} / 1$ $h$ indicated increased clarity and intensity of the $M X$ reflections with increase in the solution treatment temperature confirming precipitation of carbides. Fig 4.6 shows extensive fine carbide precipitation on the grain boundaries on a carbon extraction replica in an alloy treated for 1 hour at $975^{\circ} \mathrm{C}$ after forging. The size of the fine carbides in all the metallurgical conditions vary from 20 to $500 \mathrm{~nm}$. In all the instances, They are found to be cuboidal in shape with an fcc structure (point group $\mathrm{m} 3 \mathrm{~m}$ ) and an average lattice parameter of $4.43 \AA$ as obtained from the SADP's. The microanalytical data obtained from TEM/EDS on these particles on carbon extraction replica is given in Table 4.2. All the carbides found in various conditions of alloy 903 are MX-type Hagg compounds with M sites occupied by $\mathrm{Nb}, \mathrm{Ti}$, and $\mathrm{X}$ sites occupied by $\mathrm{C}$ or $\mathrm{N}$ or $(\mathrm{C}, \mathrm{N})$. No other carbide of any type was observed in any of the samples. Most of the carbides formed during precipitation were rich in $\mathrm{Nb}$, the average $\mathrm{Nb}$ content being 79.5 at\% and the average $\mathrm{Ti}$ content being only 20.5 at \%. However, few (trace) carbides were sometimes identified to be richer in $\mathrm{Ti}$ than Nb. These data can be seen in table 4.2. Unlike the coarse carbides formed during ingot solidification which have a distinct $\mathrm{Ti}$ rich carbide as the nuclei around which the $\mathrm{Nb}$ rich carbide grows, such inner nucleus and outer precipitate was rarely observed in the carbides formed in precipitation during the thermo-mechanical processing.

As mentioned earlier, no reliable evidence was available to determine the relative proportion of carbon and nitrogen in any of the carbides. There have been numerous studies on carbides [84-88]. TiC has a lattice parameter of $4.33 \AA$ and TiN, a value of $4.244 \AA[85]$. The lattice parameter values of $\mathrm{NbC}$ range from $4.466 \AA$ for $\mathrm{NbC}$ to 
$4.433 \AA$ for $\mathrm{NbC}_{0.72}$ [86]. While TiC and TiN are not completely soluble in each other [84], NbC and TiC are found in a continuous range of solid solution [87] with lattice parameters in the range of $4.469 \AA$ to $4.323 \AA$. $\left(\mathrm{Nb}_{0.8} \mathrm{Ti}_{0.2}\right) \mathrm{C}$ has a lattice parameter of 4.441 $\AA$.Duwez and Odell [88] have examined the NbC-TiN and NbN-TiC systems and found continuous solid solutions. Because of these complications, it is not possible to attribute a unique composition to the carbides in alloy 903. The composition as well as the latttice parameter would vary in a certain range. All of the nitrogen is expected to form nitrides and carbonitrides during solidification. Nitrides and carbonitrides have a high melting point and do not go into solution in any of the solution treatments. Hence it can be said with some certainity that the average fine carbide precipitating during heat treatments are of the type $\left(\mathrm{Nb}_{0.8} \mathrm{Ti}_{0.2}\right) \mathrm{C}$. The data on average lattice parameter (Table 4.3) and average metallic compositions also support this conclusion.

In order to examine whether MNP-type phosphides could be re-precipitated in the temperature range used for thermo-mechanical processing, some samples solution treated at $975^{\circ} \mathrm{C} / 1 \mathrm{~h}$ were aged at $850^{\circ} \mathrm{C}$ for three hours. Observations by TEM on carbon extraction replica from these samples confirmed extensive precipitation of MNP-type phosphides in clusters on grain boundaries. Figs $4.7(a, b)$ show TEM bright field images of MNP-type phosphides observed in these samples. The major difference between these precipitates and the ones found in thermo-mechanically processed material, lies in the morphology. In the samples aged for 3 hours at $850^{\circ} \mathrm{C}$, it is not possible to generally distinguish the phosphides and the carbides except by composition analysis and electron diffraction. Both have similar size and morphology. The MNP-type phosphides observed in the $850^{\circ} \mathrm{C}$ aged samples, also did not contain any internal defects. These observations are similar to the observations of Vincent [10]. These observations suggest that MNP-type phosphide particles are soft and hence get deformed during thermo-mechanical processing. The internal defects observed in the thermo-mechanically processed material is a direct consequence of the induced deformation. The carbides however are hard and remain undeformed during 

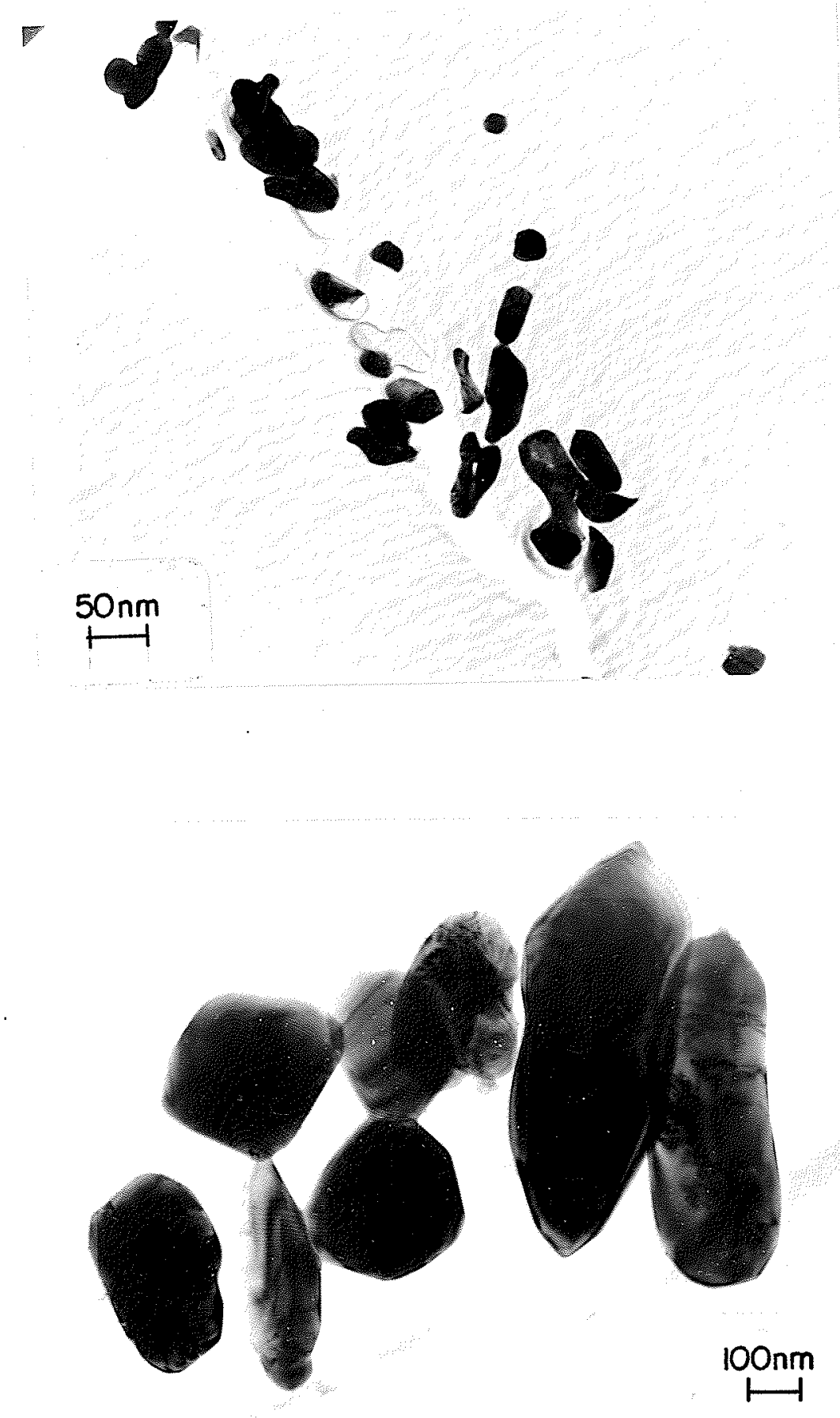

fig 4.7(a,b): A TEM bright field image of re-precipitated MNP-type phosphides on carbon extraction replica from a forged alloy solution treated at $975^{\circ} \mathrm{C} / 1 \mathrm{~h}$ and aged for three hours at $850^{\circ} \mathrm{C}$. 
thermo-mechanical processing.

As mentioned in chapter 2, few samples of forged and solution treated ring were cold rolled by $90 \%$ and then further solution treated for one hour at $1100^{\circ} \mathrm{C}$. Samples from this lot were subsequently aged at $800^{\circ} \mathrm{C}$ for 220 hours to study the precipation behavior. Few other samples from this lot were also aged for long times in the temperature range $750^{\circ} \mathrm{C}-850^{\circ} \mathrm{C}$. On long time aging two new phases were observed to precipitate, with the precipitation being generally confined to the grain boundaries. Figs $4.8(a, b)$ show optical and SEM micrographs of a sample aged for 220 hours at $800^{\circ} \mathrm{C}$. Extensive precipitation of a needle shaped phase was observed to initiate from grain boundaries and grow into the grain interior.

Carbon extraction replica's prepared from the above samples were studied by AEM. The second phase was observed to be a blocky phase outlining the grain boundaries. This phase could not be identified but is a complex intermetallic compound with $\mathrm{Fe}, \mathrm{Co}$ and $\mathrm{Nb}$ as the metallic components. The larger interplanar spacings as determined from electron diffraction patterns obtained from this phase are $5.4 \AA, 5.0 \AA, 4.5 \AA, 3.3-3.4 \AA, 3.18$ $\AA$ and $3.0 \AA$. These $d$ spacing values do not match with any of the known intermetallic phases reported in superalloys. A TEM/EDS spectrum obtained from the blocky phase is shown in fig 4.9. The TEM/EDS microanalysis of this phase is presented in table 4.4.

The needle shaped phase was identified from TEM/EDS microanalyses to be the stable delta phase which is known to precipitate in Nb-bearing superalloys during high temperature solution treatments. Morphological observations suggest the phase to form in a Widmanstatten manner. Detailed studies of the orientation relationship with matrix were not undertaken as this phase was never observed in the microstructural conditions of the alloy that were used to study weldability or in the HAZ microfissures. Though delta phase is thermodynamically stable at $840^{\circ} \mathrm{C}$, it's precipitation rate is too slow to allow for even nucleation to occur after one hour treatment at $840^{\circ} \mathrm{C}$. The delta phase solvus is approximately around $925^{\circ} \mathrm{C}$ and hence no precipitation of this phase occurs in 
solution treatments above $925^{\circ} \mathrm{C}$. Fig 4.10 shows a typical TEM/EDS spectrum obtained from the needle shaped delta phase particles. The TEM/EDS microanalysis presented in table 4.5 shows the phase to be of $A_{3} B$ type with $\mathrm{Ni}$ occupying primarily the $\mathrm{A}$ sites. The occupancy ratio of the $\mathrm{B}$ sites between $\mathrm{Nb}$ to $\mathrm{Ti}$ was found to be 3 to 2 . If $\mathrm{Ti}$ were to be the predominant occupant of the $B$ sites, then the stable equilibrium phase that forms in superalloys is the hexagonal $\mathrm{Cu}_{3} \mathrm{Ti}$-type eta phase.

To the knowledge of this author, there has not been any TEM/AEM studies reported on Incoloy 903 in the open literature. Most of the prior work $[77,78]$ has been done using SEM/EDS on bulk samples. Under SEM/BSE contrast, the fine precipitates in Incoloy 903, irrespective of whether they are phosphides or carbides appear brighter than the matrix owing to their higher niobium content. These precipitates are generally so fine that composition analysis by SEM/EDS gives only a higher niobium level with substantial nickel, iron and cobalt in all the cases, irrespective of phosphides or carbides present because of the matrix interference. Hence, no exact distinction can ever be made between the two phases, carbide and phosphide, by SEM/EDS on bulk samples. Hence, till now, owing to the higher niobium levels and irrespective of the presence or absence of some $\mathrm{Fe}$, $\mathrm{Ni}$ and $\mathrm{Co}$, the fine precipitates were always presumed to be MC-type carbides $[77,78]$. The present detailed study, however, conclusively shows that the fine precipitates can be either phosphides or carbides or a combination of both, depending on the temperatures used for the thermo-mechanical processing. This investigation shows that controlled thermomechanical processing is extremely important for obtaining the right combination of grain size distribution and controlled precipitation.

MNP-type phosphides have not been, hitherto, observed in any of the superalloys except for in the heat affected zone of an Inconel 718 weldment. The reason for their non-appearence in the other superalloys is two fold. Most of the superalloys are given solution treatments above $1000^{\circ} \mathrm{C}$. As observed in this study, the phosphides are not stable above $975^{\circ} \mathrm{C}$ and hence no phosphides would be observed in the the superalloys given final 
solution treatments in the range of $1000^{\circ} \mathrm{C}$ and above. The second factor concerns the level of phosphorus in most of the superalloys. Phosphorus and sulphur are the main residual non-metals of concern in superalloys [103]. Extensive literature is available as to the detrimental affects of sulphur on various mechanical properties as well as on the weldability of the alloy while there is a lack of literature as to the affect of phosphorus. There has been, however, one study on Inconel 600 [104] that clearly demonstrates that sulphur and phosphorus are extremely detrimental to weldability. Because of these reasons, the sulphur and phosphorus levels are kept very low in superalloys. The solution treatment temperatures are kept low in case of alloys, 901,903 and 907 because of the requirement of a necklace structure for optimum stress-rupture properties. Hence, as can be seen from this investigation, there exists a possibility of phosphide precipitation if the phosphorus content is high enough. 

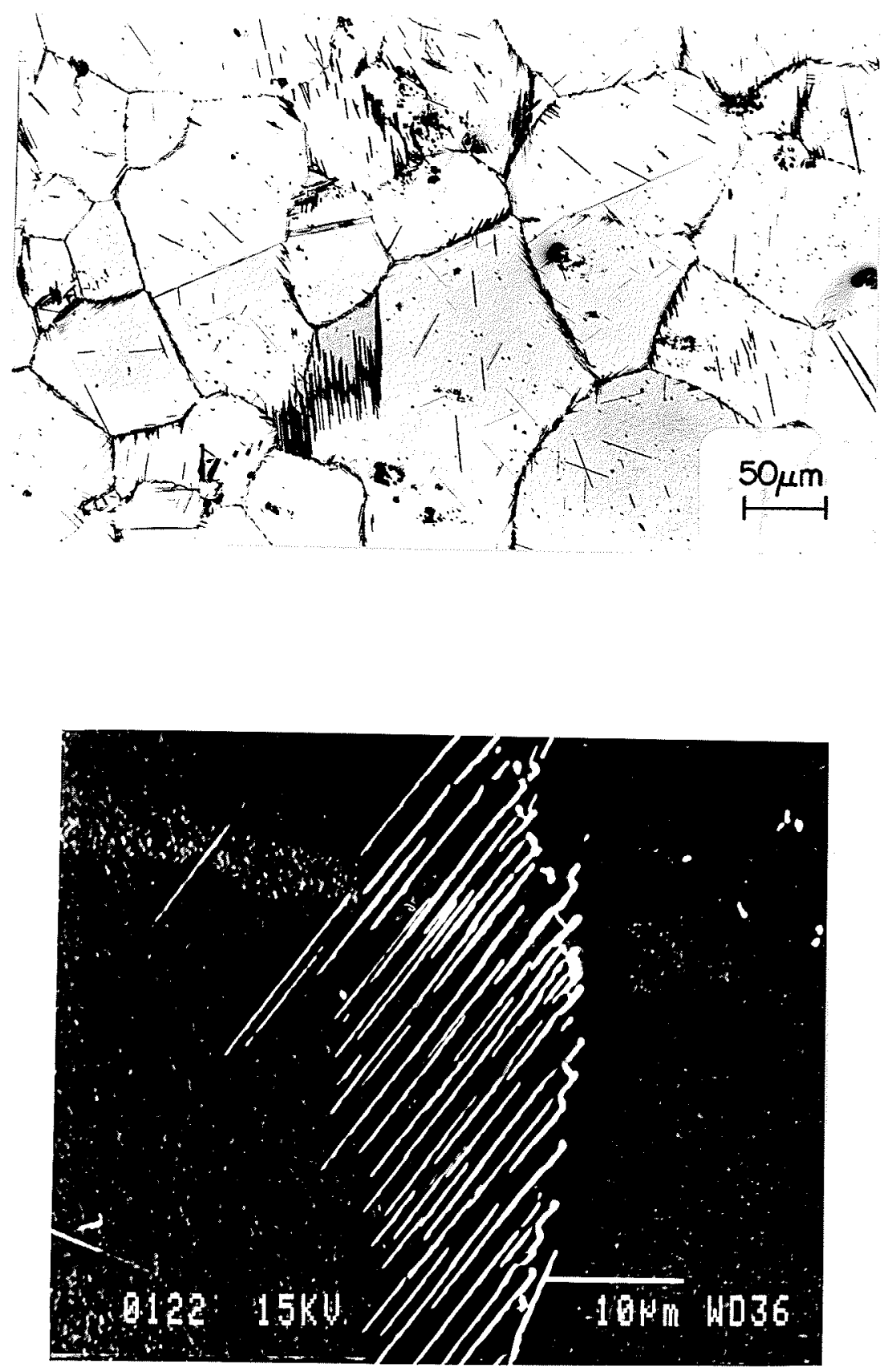

fig 4.8(a,b): Optical and SEM micrograph of Incoloy 903 aged for $220 \mathrm{~h}$ at $800^{\circ} \mathrm{C}$. 


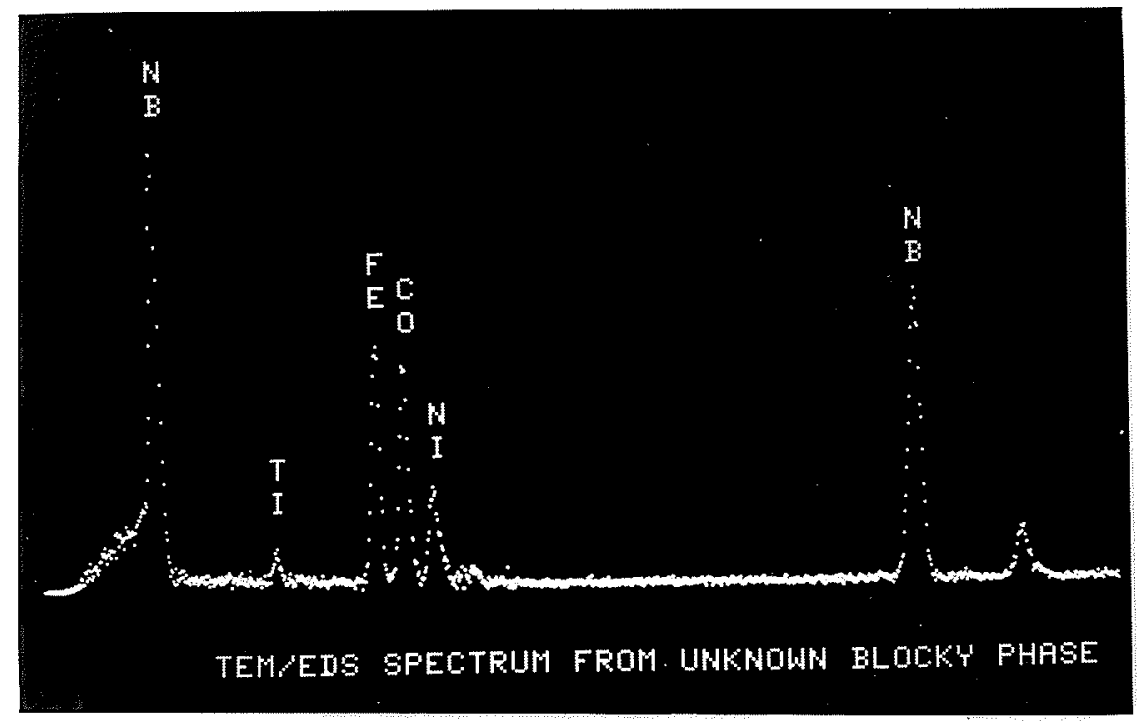

fig 4.9: A typical TEM/EDS spectrum obtained from the unidentified blocky particles in samples aged for long times in the temperature range $750^{\circ} \mathrm{C}-850^{\circ} \mathrm{C}$.

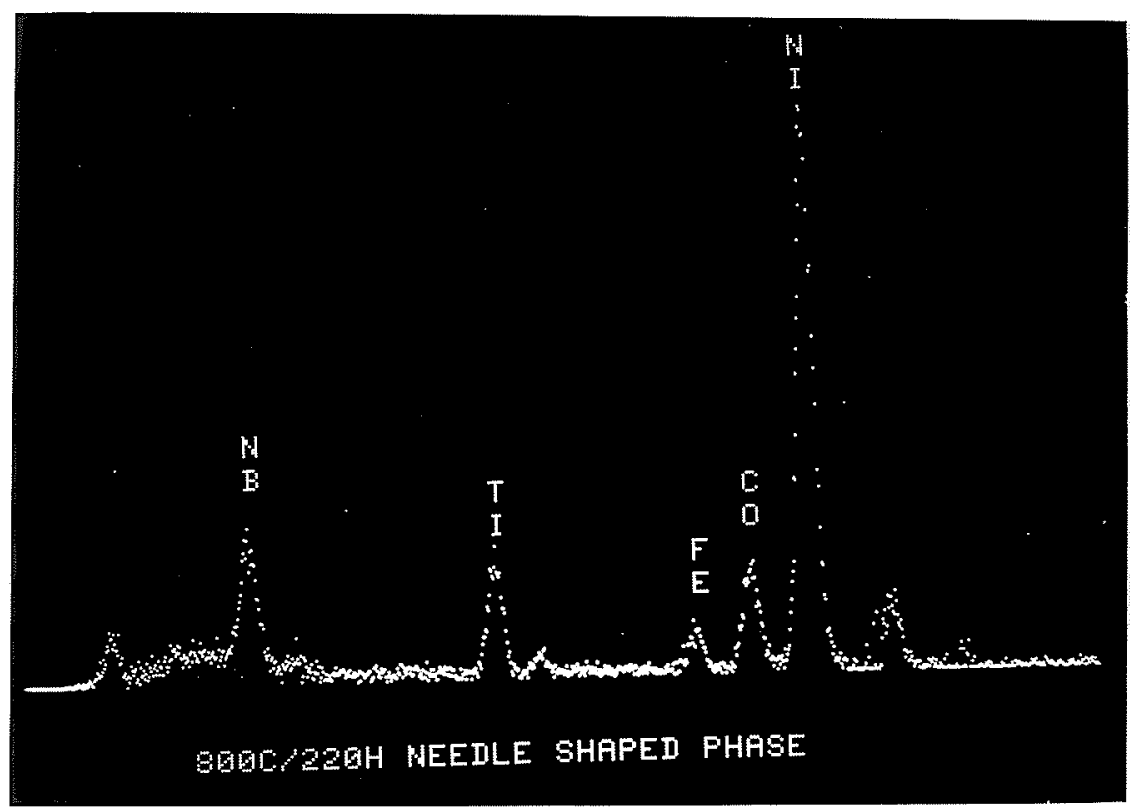

fig 4.10: A typical TEM/EDS spectrum obtained from the needle shaped phase in Incoloy 903 aged for $220 \mathrm{~h}$ at $800^{\circ} \mathrm{C}$. 
Table 4.4: Chemical composition (wt \%) of the blocky phase

\begin{tabular}{llllll}
\hline Phase & $\mathrm{Fe}$ & $\mathrm{Ni}$ & $\mathrm{Co}$ & $\mathrm{Nb}$ & $\mathrm{Ti}$ \\
Unknown & 19.2 & 7.2 & 17.4 & 54.9 & 1.3 \\
\hline
\end{tabular}

Table 4.5: Chemical composition (at \%) of the delta phase

\begin{tabular}{llllll}
\hline Phase & $\mathrm{Ni}$ & $\mathrm{Co}$ & $\mathrm{Fe}$ & $\mathrm{Nb}$ & $\mathrm{Ti}$ \\
Delta & 59.8 & 11.6 & 4.1 & 15.2 & 9.4 \\
\hline
\end{tabular}




\section{2: CHARACTERIZATION OF FUSION ZONE MICROSTRUCTURE}

\subsection{1: Introduction}

The microstructure of the fusion zone of superalloy welds typically consists of dendritic gamma matrix and the phases formed during the terminal solidification of the interdendritic liquid. The nature of the constituents formed during interdendritic terminal solidification is of fundamental importance in determining the properties of weldments of superalloys. The formation of terminal solidification constituents accompanies an extension of the temperature range over which solidification takes place. The presence of a liquid phase from which terminal solidification constituents form at a lower temperature has been shown by several investigators $[10,22,23,104,105]$ to be responsible for the hot cracking of weldments. Even a small volume fraction of terminal solidification constituent involving TCP phases can be detrimental to an alloy's resistance to hot cracking [105]. In several Nbcontaining iron-base and nickel-base austenitic superalloys, the presence of a Laves phase in the terminal solidification constituents has been observed $[75,89]$ to be detrimental to the weld metal hot cracking resistance. During solidification of the welded regions, the gamma dendrites selectively reject alloying elements such as $\mathrm{Nb}$ and $\mathrm{Ti}$ to the interdendritic liquid and in nickel-base superalloys this is accompanied by a depletion of nickel [104].

\subsection{2: Fusion Zone Microstructure}

Fig 4.11(a-c) shows optical micrographs of the fusion zone and HAZ of a case $A$ weld made on forged and solution treated ring. No evidence of exclusive fusion zone liquation cracking was observed in any of the welded samples. However, in all the samples investigated, microfissuring was observed in the heat affected zone along the grain boundaries lying perpendicular to the fusion zone. In some instances, the microfissures were observed to extend partly into the fusion zone. Microfissuring was also observed consistently in the regions of HAZ slightly removed from the fusion zone. In the fusion zone, the dendrites of 

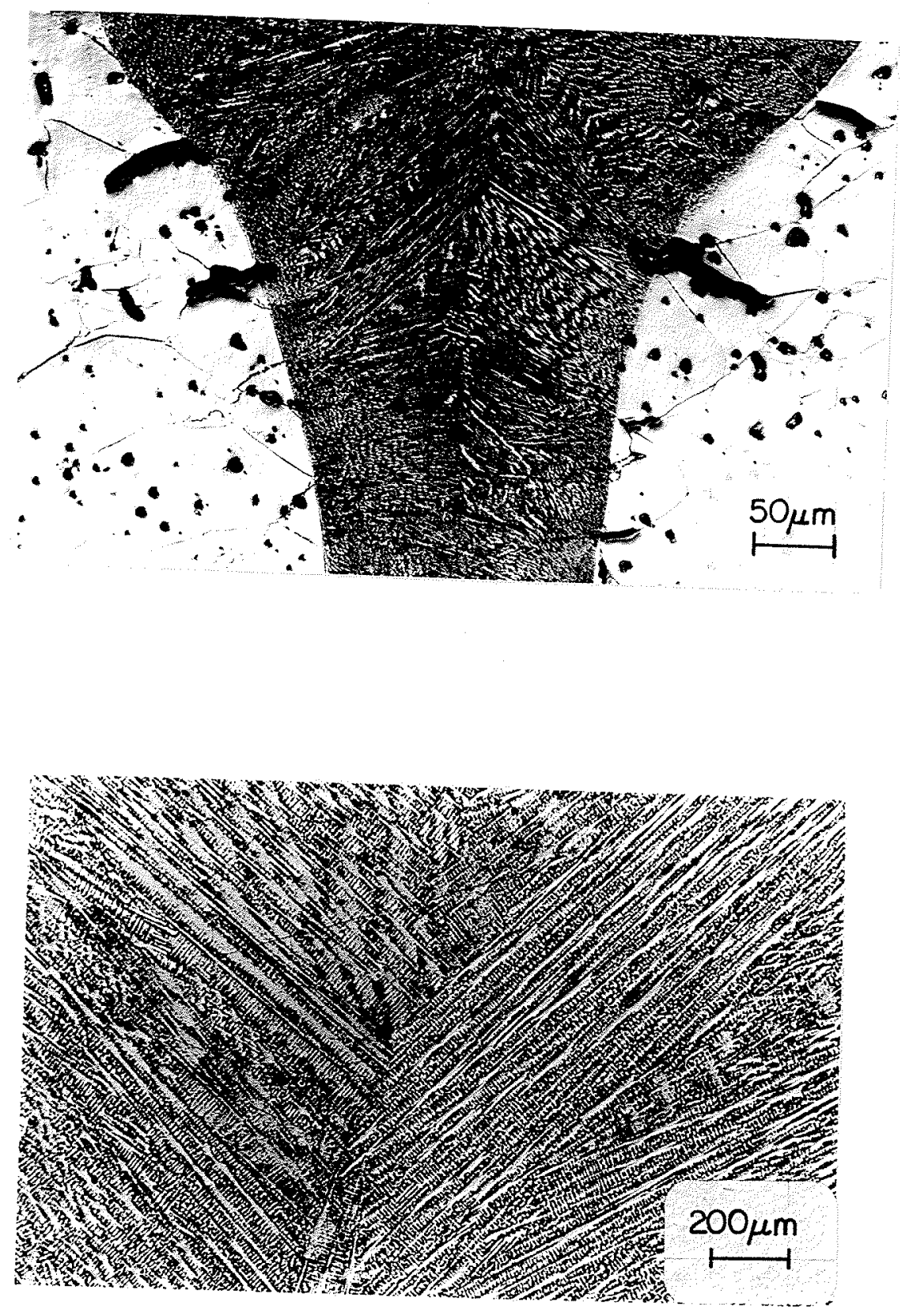

fig $4.11(a, b)$ : Optical micrographs illustrating the cellular-dendritic structure observed in the fusion zone. 
gamma phase were observed to form in a cellular-dendritic mode. The chemical composition in wt \% at the center of the austenite dendritic cores, as determined by SEM/EDS quantitative microanalyses is listed in table 4.6. It should be noted that the chemical composition of the gamma dendrites is expected to vary from the first solid to form to the last one, i.e., from the dendrite cores to the outer areas of the dendrites. However, the resolution of the SEM electron probe did not allow in the present case to determine the composition variation. Also, the composition of the gamma dendrites in the first formed dendritic cores close to the fusion line and the ones formed later close to the weld centerline are expected to be different. The composition analysis presented in table 4.6 have been obtained selectively from large dendrite areas to minimize interference from the terminal interdendritic constituents.

\subsection{3: Elemental Partitioning in the Fusion Zone:}

The weld zone is an area where complete melting and resolidification of the material occurs. A weld zone may be considered as a small casting formed under special conditions: an intensely stirred liquid pool, high temperature gradients, rapid growth rates (epitaxial), intimate contact between molten alloy and the mold including partial melting of the mold [2]. Hence, a fundamental solidification parameter that can be determined from the above data is the elemental distribution coefficient, $k$. Under equilibrium conditions and by neglecting undercooling at the dendrite tips, the first regions to solidify from the melt, i.e., the dendrite core areas are of composition $\mathrm{kC}_{0}$, where $\mathrm{C}_{0}$ is the nominal composition and $k$ is the equilibrium partition or distribution coefficient, defined as the ratio of the composition of the solidus to liquidus at the temperature, T. The ratio between the dendrite core composition and the nominal composition of the alloy will thereby yield the distribution coefficient, $k$, at the beginning of the solidification process. The distribution coefficient describes the extent and direction of microsegregation to be expected during the solidification of the alloy $[3,4]$. Values of $k$ less than unity indicate that dendrite cores 
are depleted in a particular alloying element relative to the interdendritic regions while values greater than unity suggest an enrichment. A value of unity for $k$ indicates that no dendritic microsegregation has occurred. Furthermore, an increase in the amount of alloying elements with a distribution coefficient less than unity causes a lowering of the liquidus and the solidus temperatures [3,4]. The reverse occurs for alloying elements with a value of $k$ greater than unity. The initial distribution coefficients for various alloying elements are calculated and presented in table 4.7 .

The microanalyses of dendrites in this study indicate that the values of $k$ for $\mathrm{Nb}$ and $\mathrm{Ti}$ are far less than unity while those for $\mathrm{Fe}$ and $\mathrm{Co}$ are slightly greater than unity. The values of distribution coefficients of $\mathrm{Ni}$ and $\mathrm{Al}$ are observed to be slightly less than unity.

Niobium segregation plays an important role in the formation of terminal solidification constituents in the form of gamma/Laves eutectic or gamma/carbide eutectic. The value of $k_{N b}$ observed in this study is very similar to that stated by Knorovsky et al. [106] for $\mathrm{Nb}$ in Inconel $718\left(\mathrm{k}_{N b}=0.5\right)$ and by Cieslak et al. [107] for alloy $625\left(\mathrm{~K}_{N b}=0.54\right)$. Comparing the values of $k$ obtained in this study with those reported in various other superalloys [105,-107], it seems that the dendrite cores are always enriched in $\mathrm{Fe}$ and $\mathrm{Co}$ while being depleted in $\mathrm{Nb}$ and $\mathrm{Ti}$. The major difference observed in Incoloy 903 is with respect to the partitioning of $\mathrm{Ni}$ during solidification. In many superalloys ( $\mathrm{C}-4, \mathrm{C}-22$, C-276 [105] and Inconel 718 [106]) the dendrite core is observed to be enriched in $\mathrm{Ni}$. However, in the 900 series superalloys, based on the Fe-Ni-Co ternary, Ni has a distribution coefficient of less than unity as observed in the present investigation on Incoloy 903 and by Cieslak et al. [108] in their study on Incoloy 909.

No attempt was made in this investigation to analyze the concentrations of $\mathrm{P}$ and $\mathrm{Si}$ in the gamma dendrites as that would have required extremely large acquisition times to obtain statistically acceptable $x$-ray counts, because the concentration of $P$ and $S i$ in the base metal is only 0.004 wt $\%$ and 0.07 wt \% respectively. However, it is essential to discuss the partitioning behavior of these elements during solidification. $P$ appears to play 
Table 4.6: Chemical composition (wt \%) of the gamma dendrite cores

\begin{tabular}{llllll}
\hline Fe & Ni & Co & Nb & Ti & Al \\
42.98 & 37.57 & 15.69 & 1.70 & 1.07 & 0.90 \\
\hline
\end{tabular}

Table 4.7: Partitioning coefficients of various alloying elements

\begin{tabular}{lll}
$\mathrm{k}_{N b}=0.58$ & $\mathrm{k}_{T i}=0.76$ & $\mathrm{k}_{A l}=0.95$ \\
$\mathrm{k}_{F^{c}}=1.06$ & $\mathrm{k}_{C o}=1.05$ & $\mathrm{k}_{N i}=0.97$ \\
\hline
\end{tabular}


a significant role in HAZ microfissuring in Incoloy 903 while increased $\mathrm{Si}$ levels are known to promote the formation of the Laves phase as a terminal solidification constituent. Laves phase formation from the terminal interdendritic liquid is known to promote $H A Z$ microfissuring during welding. It has been reported that both $\mathrm{P}$ and $\mathrm{Si}$ have distribution coefficients far less than unity in nickel and iron-base binary systems [109]. The k value of $P$ in Ni-P and Fe-P binary system is 0.06 and $0.06-0.13$ respectively [109]. The $k$ value of $\mathrm{Si}$ in Ni-P and Fe-P binary is 0.62 and $0.5-0.61$ respectively [109]. Hence, the $k$ values of $\mathrm{P}$ and $\mathrm{Si}$ in Incoloy 903 based on Fe-Ni-Co ternary system is expected to be of the same magnitude.

Special analytical techniques other than those used in the present study are required to determine the value of the distribution coefficient, $k$, of carbon. It has been mentioned by Knorovsky et al. [106] that the distribution coefficient of carbon in alloys similar to Inconel 625 is approximately 0.2 . Thompson et al. [110] in their study on Inconel 718 by Auger electron spectroscopy have experimentally confirmed that carbon segregates into the interdendritic liquid. Thus, it is reasonable to assume that in Incoloy 903, during solidification, an extensive segregation of carbon, phosphorus and silicon atoms occurs into the interdendritic liquid.

\subsection{4: Fusion Zone Secondary Solidification Constituents:}

The major interdendritic constituent observed in the fusion zone consisted of sheets with distinctive arrow head and lobed morphologies. Fig 4.12(a-c) shows a TEM micrograph of interdendritic solidification constituents formed in the fusion zone and extracted on a carbon replica. The inset in the fig 4.12(b) shows a diffraction pattern and fig $4.12(d)$ shows a typical TEM/EDS spectrum obtained from these sheets. Electron diffraction and TEM/EDS x-ray microanalyses indicate the sheets to be cubic (fcc), MC carbides, rich in niobium with a lattice parameter of $4.43 \AA$. Trace analysis indicates the carbide dendrite arms to be parallel to the cube axes with the growth terminating with the formation of 
$\{111\}$ facets. This is consistent with the fact that in cubic alloys, the cube axis directions $<100>$ are the preferred dendrite growth directions $[3,4]$. The typical, distinctive arrow head morphology of the carbide sheets, fig 4.12(c), is developed as a consequence of the termination of growth by $\{111\}$ facets. The average chemical composition of the metallic component of the carbides as determined by TEM/EDS is $85.8 \mathrm{wt} \% \mathrm{Nb}$ and $14.2 \mathrm{wt}$ $\% \mathrm{Ti}$. A comparison with the results on base metal microstructural characterization presented in section 4.1 , shows that the interdendritic carbides are similar in crystal structure, lattice parameter, and composition to the carbides observed on the grain boundaries in the base metal.

Apart from the carbides, the only other phase observed in the fusion zone, in trace amounts, is the hexagonal $M g Z n_{2}$-type Laves phase with a lattice parameter of $a=4.76$ $\AA$ and $c=7.76 \AA$. The Laves phase particles were easily identifiable owing to their being heavily faulted. They were generally observed to form on the edge of the carbides. Fig 4.13(a) shows a TEM micrograph of a small Laves phase particle observed on the edge of a carbide and the inset shows a [1100] zone diffraction pattern obtained from these particles. Fig 4.13(b) shows a typical TEM/EDS spectrum from a Laves phase particle. The chemical composition of the Laves phase in wt \% as obtained from TEM/EDS microanalyses is given in table 4.8 .

A silicon peak was consistently observed in all the spectra obtained from the Laves phase particles. Silicon has been reported to promote the formation of Laves phase in ternary transition metal alloys [111] and as a solid state transformation product in other superalloys [112,113]. Silicon has also been associated with a Laves type solidification constituent in alloy A-286 [114], in Inconel 718 [89,106,108] and in Incoloy 909 [108]. The microanalyses of the Laves phase particles obtained in the present study show that the expected stoichiometry $A_{2} B$ is not met in Incoloy 903 and there is very little partitioning of $\mathrm{Ti}$ to them. These observations are similar to the observations by Cieslak et al [108] on Inconel 718 and Incoloy 909 . They found minimal partitioning of $\mathrm{Ti}$ to the Laves phase as 


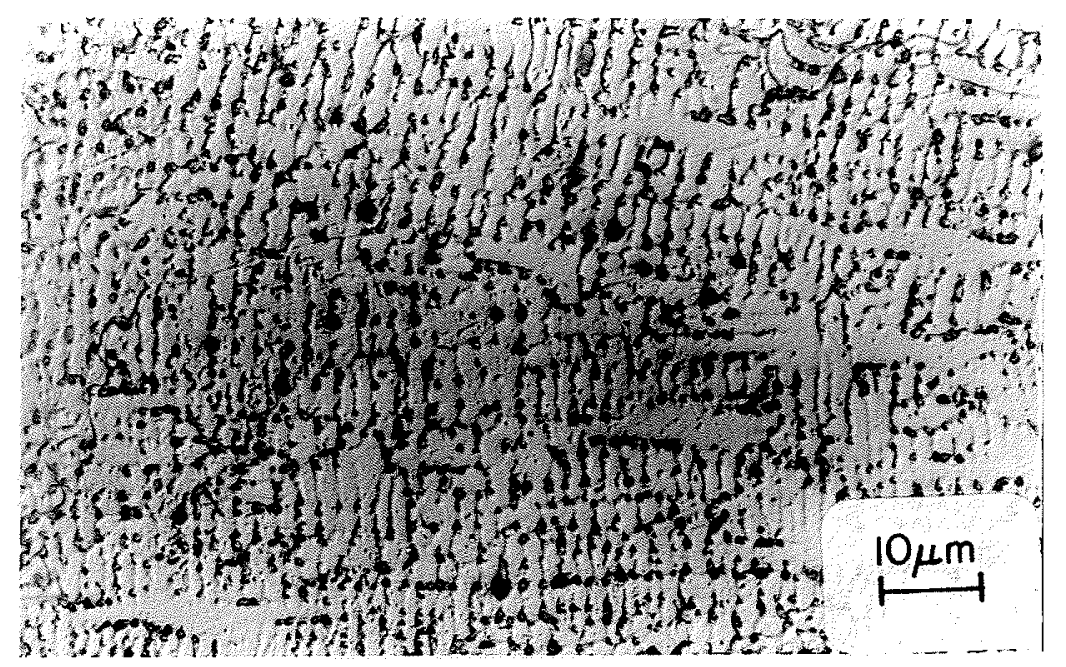

fig 4.11 (c): Optical micrograph illustrating the cellular-dendritic structure observed in the fusion zone.

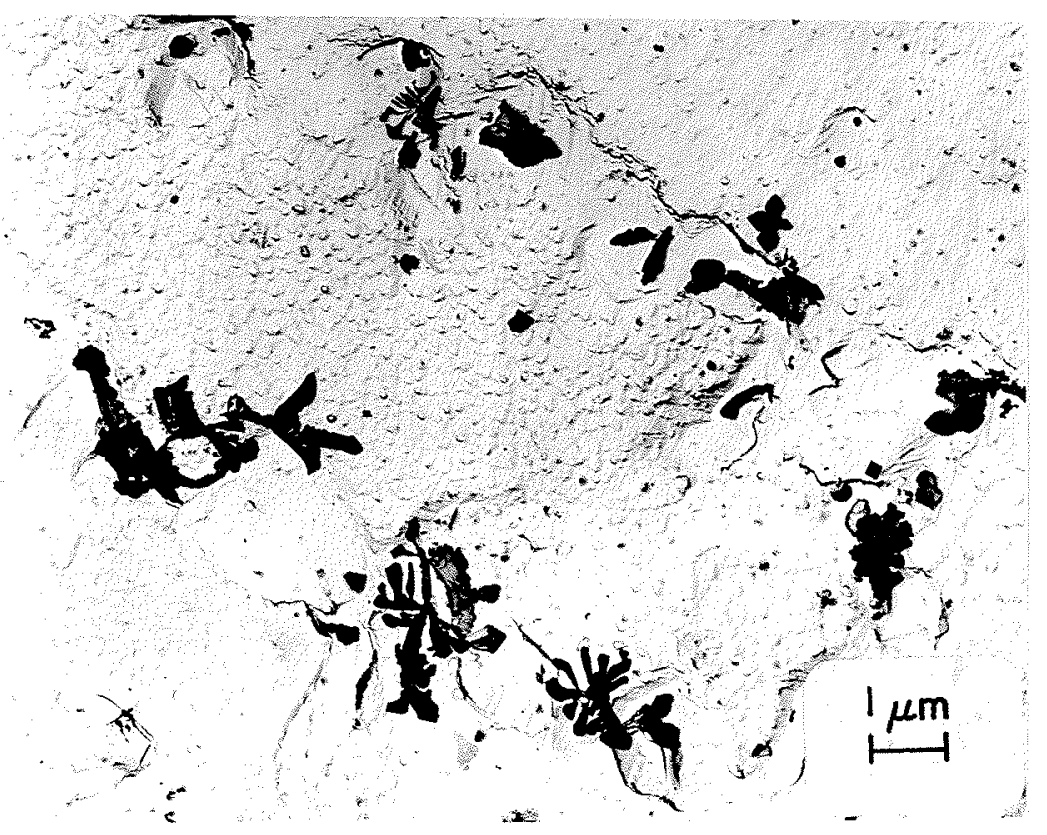

fig 4.12(a): Low magnification TEM micrograph of interdendritic carbides from fusion zone extracted on a carbon extraction replica. 


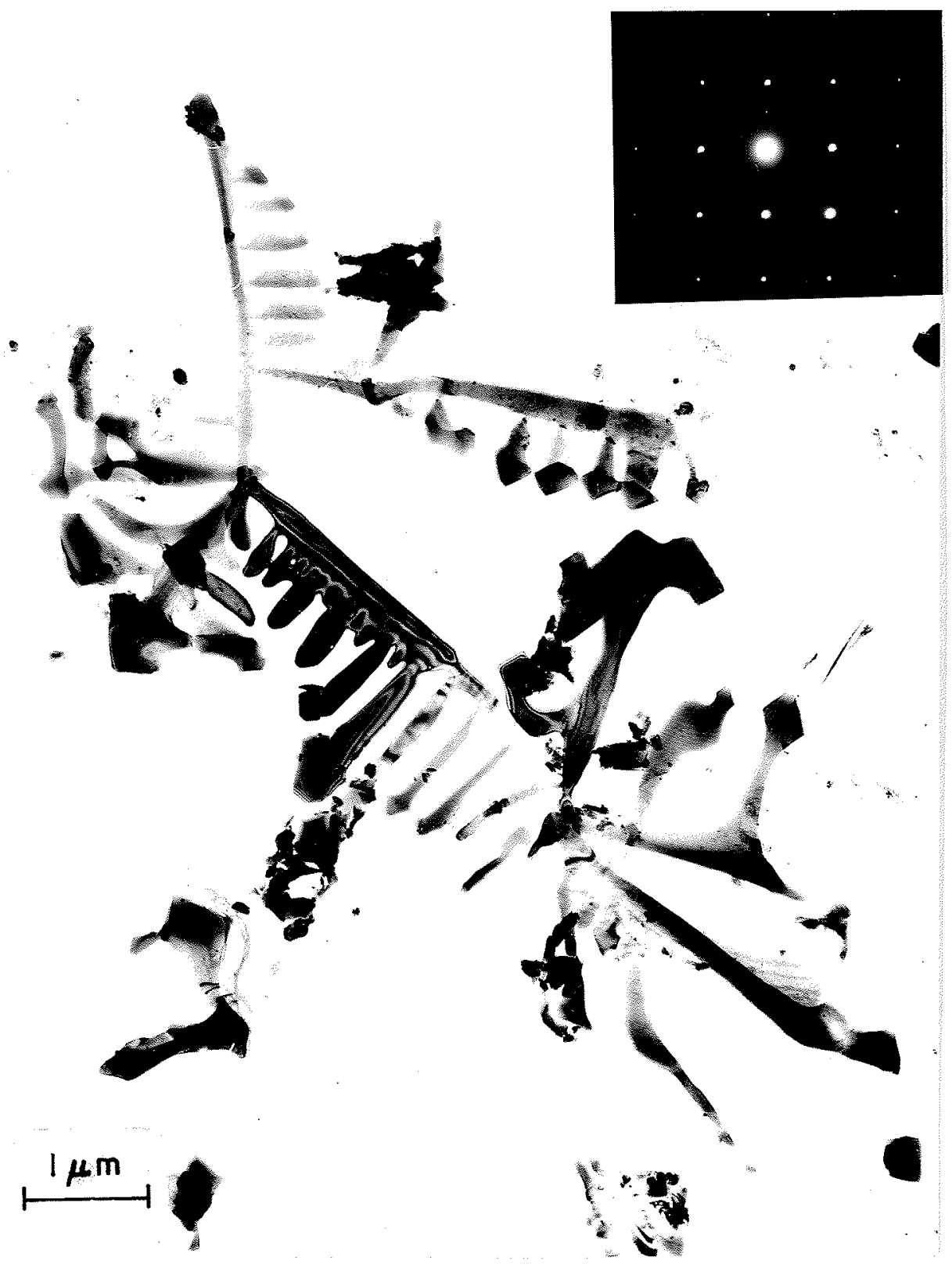

fig 4.12(b): TEM micrographs of interdendritic carbides from fusion zone extracted on a carbon extraction replica. The inset in figure 4.12(b) shows a [001] zone diffraction pattern obtained from a carbide. 


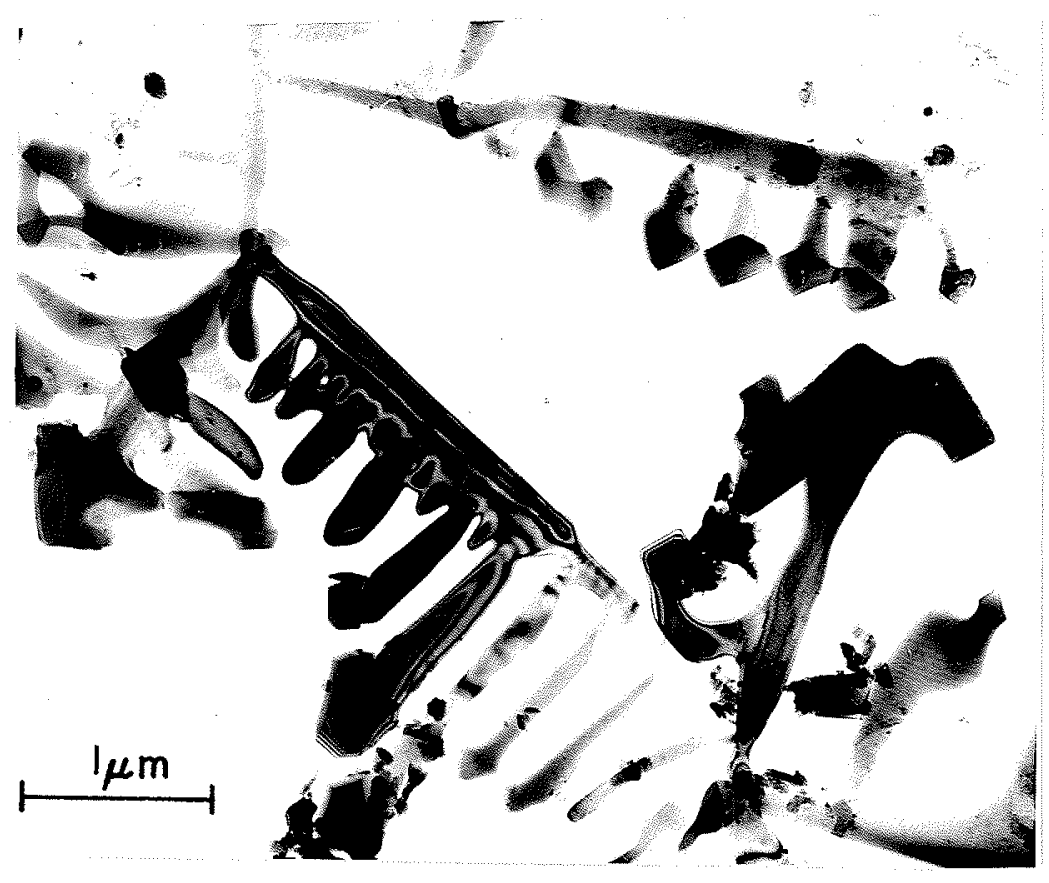

fig 4.12(c): Illustrating the characteristic arrow head and lobed morphology of interdendritic carbides.

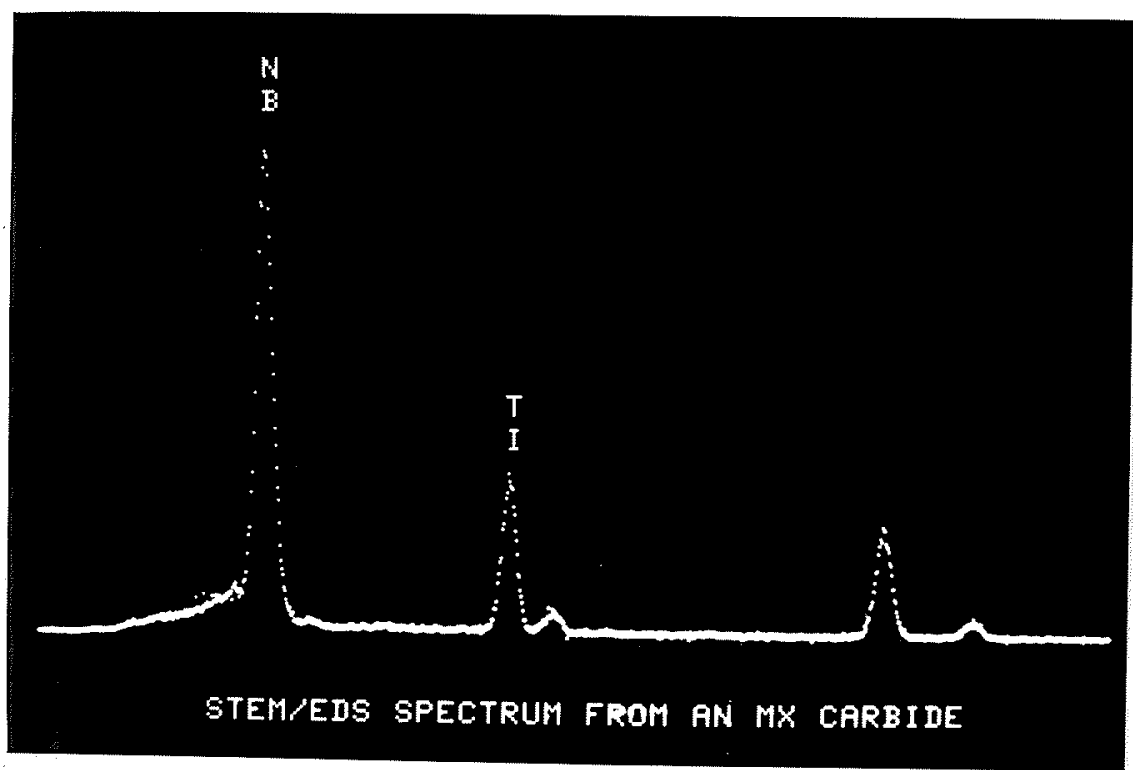

fig 4.12(d): A typical TEM/EDS spectrum obtained from an interdendritic carbide sheet. 


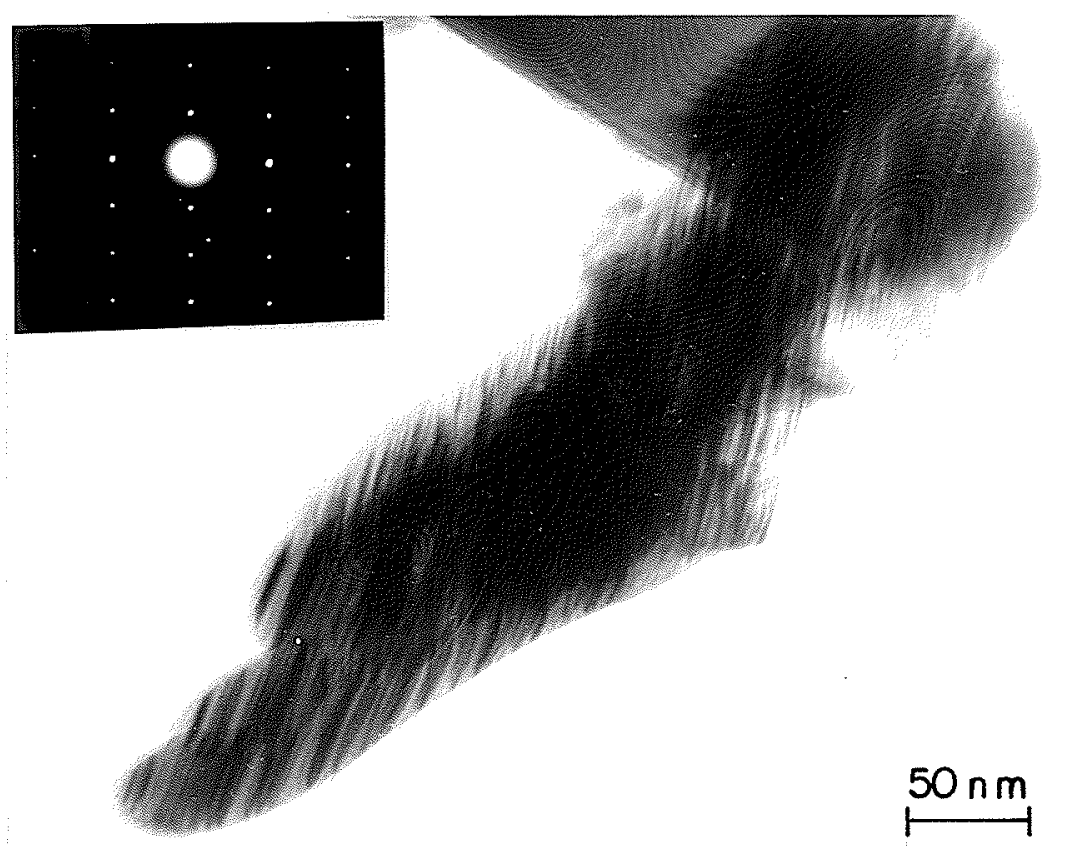

fig 4.13(a): TEM micrograph of a Laves phase particle observed on the edge of a carbide sheet. The inset shows a [1100] zone diffraction pattern obtained from a Laves phase.

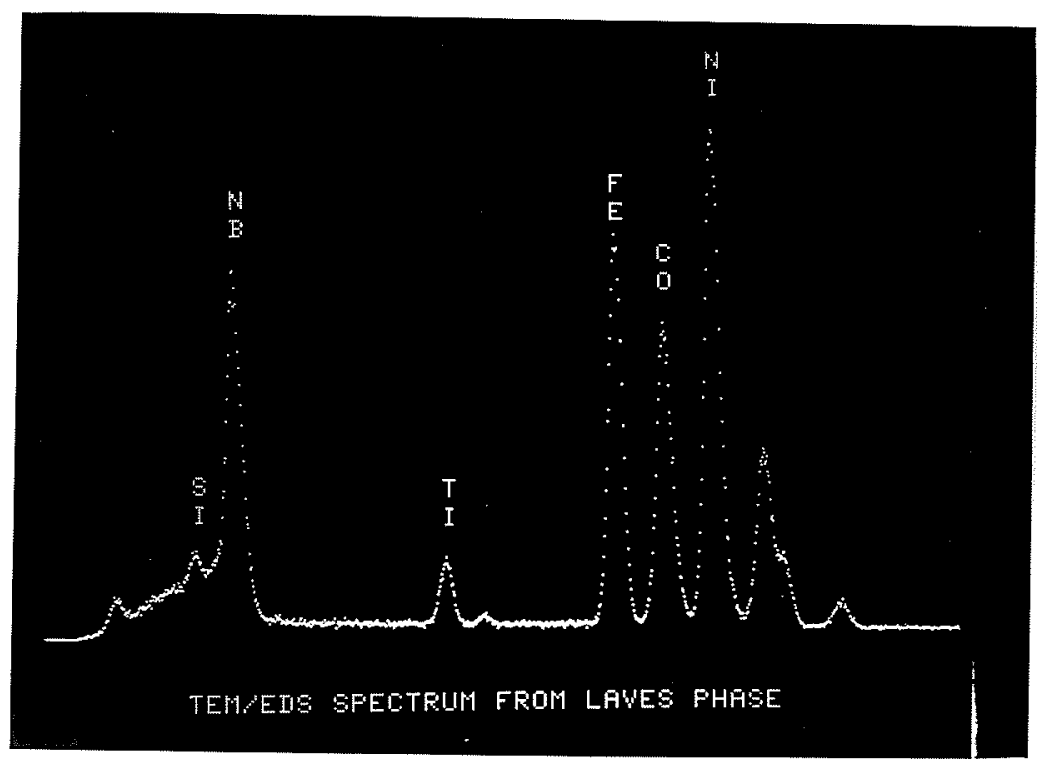

fig 4.13(b): A typical TEM/EDS spectrum obtained from a Laves phase particle. 
well as increased total atomic fraction of A type atoms. Another similarity found between the present study and that of Cieslak et al. [108] is the predominance of Ni among A type atoms as opposed to $\mathrm{Fe}$ and $\mathrm{Co}$ atoms. A possible reason could be that $\mathrm{Fe}$ and $\mathrm{Co}$ have $k$ values greater than unity and as such segregate in the gamma dendrites while Ni with a $k$ value marginally less than unity segregates into the interdendritic liquid in Incoloy 903 and Incoloy 909.

Table 4.8: Chemical composition (wt \%) of the Laves phase

\begin{tabular}{llllll}
\hline $\mathrm{Fe}$ & $\mathrm{Ni}$ & $\mathrm{Co}$ & $\mathrm{Nb}$ & $\mathrm{Ti}$ & $\mathrm{Si}$ \\
21.0 & 28.2 & 15.9 & 30.4 & 2.9 & 1.6 \\
\hline
\end{tabular}

The first solid to form from the weld pool at the liquidus temperature is in the form of gamma dendrites of composition $\mathrm{kC}_{0}[3,4]$. As the temperature decreases, the composition of the liquid in front of the growing dendrites gets enriched with solutes (for which $k<1$ ) that are continually being rejected into the interdendritic liquid. The alloy content of the forming solid also gradually increases in elements for which $k<1$. Secondary solidification constituents start forming when the primary solidification phase can no longer accommodate the alloy concentration imposed on it by solute segregation, i.e, when the solubility of the alloying elements in the liquid is exceeded. These phases start forming from the supersaturated interdendritic liquid since they are more thermodynamically and structurally capable of relieving the supersaturation. The formation of secondary solidification constituents can be predicted from a consideration of the phase diagram of the alloy. In the case of nickel-base alloys, several secondary solidification constituents are possible, including carbides, borides, nitrides, $\gamma^{\prime}$, and a host of other intermetallic phases. When the effective solidus temperature is reached, the remaining liquid transforms by an 
invariant reaction, which generally is a eutectic transformation.

In Incoloy 903, the gamma dendrites form first at the liquidus temperature and during their continued formation predominantly reject $\mathrm{Nb}, \mathrm{C}, \mathrm{Si}, \mathrm{P}$ and $\mathrm{Ti}$ into the interdendritic liquid. Gamma/(Nb,Ti)C constituents start forming from the enriched interdendritic liquid once the interfacial composition satisfies the three-phase non-invariant reaction $L \longrightarrow$ gamma $+(\mathrm{Nb}, \mathrm{Ti}) \mathrm{C}$. It has been shown by Knorovsky et. al. [106] that in Inconel 718, the gamma/carbide monovariant eutectic reaction initiates at a much higher temperature than the gamma/Laves eutectic. The data on reaction temperatures in steels also indicates that the $\gamma / \mathrm{NbC}$ reaction intiates at a higher temperature than the $\gamma /$ Laves eutectic reaction. If the nominal composition of the alloy is such that the (gamma $+(\mathrm{Nb}, \mathrm{Ti}) \mathrm{C}+$ Laves) phase field is stable at the solidus temperature, then the solidification terminates with the invariant reaction, $\mathrm{L} \longrightarrow$ Gamma $+(\mathrm{Nb}, \mathrm{Ti}) \mathrm{C}+$ Laves [115].

The formation of MC type carbides as the major secondary solidification constituent merits some discussion. The total volume fraction of the carbides in $\mathrm{Nb}$-containing nickeliron-base superalloys has been reported $[110]$ to be a function of the $\mathrm{C}$ to $\mathrm{Nb}$ ratio. $\mathrm{An}$ approach based on the gamma-NbC-Laves pseudo-ternary phase diagram has been utilized [115] to explain the dependence of relative volume fraction of the carbides and Laves phase observed in as-cast Inconel 718 alloys with varying carbon contents. In a study on Incoloy 903, Baeslack et al $[77,78]$ have reported the formation of gamma/Laves phase eutectic as the major component based on similar observations in nickel-iron-base alloys like Inconel 718 and Incoloy 909 . They also reported the formation of some fine carbides from a second eutectic reaction during the final stages of solidification. However, no definitive evidence was provided in either of the studies [77,78] for the occurrence of Laves phase by electron diffraction and TEM/EDS or by x-ray diffractometry. As can be observed in TEM micrographs presented in figs $4.12(a, b)$, the thickness as well as the width of the carbide sheets are generally in the range of $150 \mathrm{~nm}$. The analysis of these carbides by SEM/EDS will never be accurate owing to the generation of a larger $x$-ray excitation volume than 
the carbide volume by the electron beam in a SEM.

The nominal composition of Incoloy 903 is such that the minor phases observed in wrought and thermo-mechanically processed material are the large $M X$ carbides and/or carbonitrides formed during ingot solidification and fine MC carbides formed from the solid state reactions occurring during processing. As observed from the section on microstructural characterization of Incoloy 903, MNP-type phosphides and orthorhombic $A_{3} B$-type delta phase can also precipitate under certain processing and/or heat treatment conditions. However, no Laves phase has been observed to form in Incoloy 903 during thermo-mechanical processing or heat treatments. Hence, it may be concluded that the composition of the Incoloy 903 lies in the stable (gamma $+(\mathrm{Nb}, \mathrm{Ti}) \mathrm{C}$ ) phase field. This explains the formation of $(\mathrm{Nb}, \mathrm{Ti}) \mathrm{C}$ carbides as a major secondary solidification constituent during solidification of the alloy.

Studies on austenitic stainless steel high energy density welds have shown that in addition to a refined microstructure, both changes in solidification behavior and changes in solid state transformations, than those observed in gas tungstun arc (GTA) welds occur. However, as noted in the discussion on partitioning behavior, the partitioning of various elements in the fusion zone of the electron beam welds used in the present investigation are in close agreement with those obtained in other superalloys by studies of samples after DSC analyses and/or GTA welds. Since, the partitioning of various elements determines the formation of secondary solidification constituents in the fusion zone and in the HAZ microfissures, the same phases should be observed in electron beam and GTA welds of Incoloy 903 despite the high energy density in the electron beam welds.

In austenitic stainless steels, the formation of a $\mathrm{NbC} /$ austenite eutectic has been observed [116] as the terminal solidification structure. The metal physics of nickel-ironbase-superalloys can be considered as an extension of stainless steel. In Hastelloy C-4, TiC has been observed [105] as the terminal solidification constituent. It has been shown [107] in Inconel 625 that the proportion of carbides to Laves phase in the solidification structure 
can be varied to a great extent by altering the amounts of carbon and silicon. It was observed that carbon ( $0.038 \mathrm{wt} \%$ ) promoted the formation of the gamma/MC eutectic and eliminated the gamma/Laves eutectic when silicon ( 0.03 wt \%) was not present as an intentionally alloyed element [107]. Cieslak et al. [107] have also observed the formation of both $\mathrm{NbC/gamma} \mathrm{euctectic} \mathrm{and} \mathrm{gamma/Laves} \mathrm{eutectic} \mathrm{in} \mathrm{Inconel} 625$ when both carbon and silicon were alloyed to a high level. Elsewhere, Cieslack continuing his studies on the welding metallurgy of Inconel 625 has reported that addition of carbon to $\mathrm{Nb}$-bearing alloys was observed to promote the formation of gamma/MC eutectic constituent and additions of silicon were observed to promote gamma/Laves phase eutectic constituent [117]. Addition of carbon to IN-519 have been reported [75] to cause an increase in the amount of gamma/NbC constituent at the expense of gamma/Laves phase constituent.

Hence, high silicon and low carbon levels appear to be essential for the occurrence of gamma/Laves phase as the terminal solidification constituent in Nb-containing nickeliron base superalloys. In Incoloy 909 where there is a four-fold increase in silicon and elimination of carbon as compared with Incoloy 903, only the terminal eutectic reaction of gamma/Laves phase is reported [108]. However, Incoloy 903 has a low silicon content $(0.07 w t \%)$ and a comparatively higher carbon level of 0.028 wt \%. Hence, the gamma/(Nb, Ti)C constituent by far predominates over the gamma/Laves phase constituent in the solidification microstructure of the fusion zone of the weldments. Because of the low $k$ value of silicon, the interdendritic liquid during solidification of Incoloy 903 gets progressively enriched in $\mathrm{Si}$ with the continued growth of gamma dendrites. Furthermore, the onset of $\gamma / \mathrm{NbC}$ monovariant eutectic reaction and subsequent depletion of carbon lead to an increase in the relative proportion of $\mathrm{Si}$ to $\mathrm{C}$ in the interdendritic liquid. High $\mathrm{Si}$ to $\mathrm{C}$ ratio, as discussed above, promotes the formation of the Laves phase at the expense of carbides. Hence Laves phase can form in trace amounts at the edges of interdendritic carbides, as experimentally confirmed in the present investigation, during the final stages of solidification of the interdendritic liquid. 


\section{3: CHARACTERIZATION OF HAZ MICROSTRUCTURES}

\subsection{1: Introduction}

The experimental results presented on $\mathrm{HAZ}$ microstructures are classified under three major categories. The majority of the results in the following section pertains to the observations on HAZ microfissuring in the forged and solution treated ring where (a) most of the grains observed were primarily large warm worked grains, i.e the ring samples on which the bead on plate welds were performed approximately along a constant diameter (welding was performed under case $A$ welding conditions along a straight line on a $8-10$ $\mathrm{cm}$ section of the ring perpendicular to the radius of the ring) (b) bead on plate welds performed on the ring based on some of the welding conditions obtained according to the factorial design outlined in chapter 2 (case B), but only in the cases where the base metal microstructure contained significant amount of fine recrystallized grains as well as warm worked grains apart from extensive microfissuring in the HAZ.

The second part of the section describes the results on the bead on plate welds performed on the as-forged samples solution treated for one hour at $975^{\circ} \mathrm{C}$ (case A conditions). $975^{\circ} \mathrm{C}$ was considered because, it was observed after initial experimentation that neither the duplex structure nor many MNP phosphides existed after an hour's solution treatment at this temperature. The third part of the section pertains to the results of observations on the HAZ microstructures on bead on plate electron beam welds performed on the forged and solution treated ring samples further solution treated at $1100^{\circ} \mathrm{C}$ in the time range of $1 / 2$ to 8 hours (case $A$ conditions). The solution treatments at $1100^{\circ} \mathrm{C}$ for varying times were performed to verify the two reported general trends (a) that high temperature solution treatments increase microfissuring susceptibility and (b) that a linear relationship exists between microfissuring susceptibility and increasing grain size.

The results are presented without extensive reference to different solution treatment conditions because it was observed that the only difference was in the terms of the com- 
position of the solute enriched areas, that form by liquid film migration (LFM), between forged and solution treated ring and the as-forged sampled solution treated for an hour at $975^{\circ} \mathrm{C}$. This is obviously due to the difference in the nature of precipitates undergoing constitutional liquation on the HAZ boundaries. The phases observed in HAZ microfissures were found to be the same irrespective of the solution treatment condition. It is to be noted that while the phases observed in the HAZ microfissures were the same irrespective of the solution treatment temperature, the total volume fraction of the resolidified liquid would certainly vary.

The order in which the experimental results are presented and discussed in this dissertation is at variance with the normal manner of presenting the optical and scanning electron microscopy results first, followed by analytical electron microscopic observations. However, the nature of the results obtained in this investigation compels a reversal of the normal order so as to provide an easy, continuous and coherent presentation of results and discussion without redundance. So, AEM results of phases extracted from grain boundaries and microfissures in HAZ is presented first followed by optical and SEM observations. Once the phases formed in the HAZ were characterized in terms of morphology, crystal structure and composition, and related to the kinetics and nature of their formation, it was found that the optical and SEM observations could be lucidly explained just based on the morphological features.

\subsection{2: Heat Affected Zone Microstructure-AEM observations}

\subsubsection{1: Forged and Solution Treated Ring:}

Fig 4.14 shows a low magnification TEM micrograph of the fusion zone and the heat affected zone extracted on a carbon replica from a weld made under case $A$ conditions. A large microfissure can be observed in the HAZ. Fig 4.15(a-c) shows a TEM micrograph of HAZ grain boundary microfissure. Figs $4.15(b, c)$ show areas of the composite fig 4.15(a) at a higher magnification. Extensive thin sheets of continuous and/or semi- 
continuous phases as well as interdendritic carbides with their characteristic arrow head and lobed morphologies can be observed. Some microfissures had numerous fine cuboidal precipitates apart from the interdendritic carbide sheets and thin solidified films. Fig 4.15(d) shows an interdendritic carbide sheet in a microfissure and fig 4.15(e) shows fine carbide precipitation aside a microfissure. It was also observed that the solidification constituents form mostly on one side of the microfissure. Hence, it appears that in the stage when liquid is present, microfissuring generally occurs by decohesion across one of the solid-liquid interfaces.

TEM/EDS and electron diffraction analyses was carried out on all the particles in the microfissures and the phases are labelled in the fig 4.15(a). It was found that majority of the particles are MC carbides with a similar lattice parameter and composition as of the carbides observed in the base metal and fusion zone. The carbides, as can be seen from the figs $4.15(b-e)$, were found in and/or adjacent to the microfissures in three different morphologies, viz., arrow head and lobed interdendritic sheets, thin long continuous and/or semi-continuous solidified films and fine cuboidal precipitates.

Apart from the carbides, Laves phase was detected in trace amounts in the microfissures at the edges of interdendritic carbides and also intermixed with carbides in the thin solidified films. A few particles of another unidentified phase with $\mathrm{Fe}, \mathrm{Co}$ and $\mathrm{Nb}$ as metallic components were also observed in the microfissures intermixed with the carbides in the thin solidified films (The only positive evidence to this observation is based on the finger printing technique using TEM/EDS spectra). It was observed to be the same phase that was observed to form on grain boundaries of samples aged isothermally for a long time in the temperature range, $750^{\circ} \mathrm{C}-850^{\circ} \mathrm{C}$. A couple of particles of a phase with hexagonal crystal structure and lattice constants of $a=3.27 \AA$ and $c=11.44 \AA$ were also observed in the HAZ of some samples. TEM/EDS spectra obtained form these particles suggest it to be based on titanium carbosulphide with substantial substitution of niobium for titanium. Figs $4.16(a, b)$ show a diffraction pattern and a typical TEM/EDS spectrum 


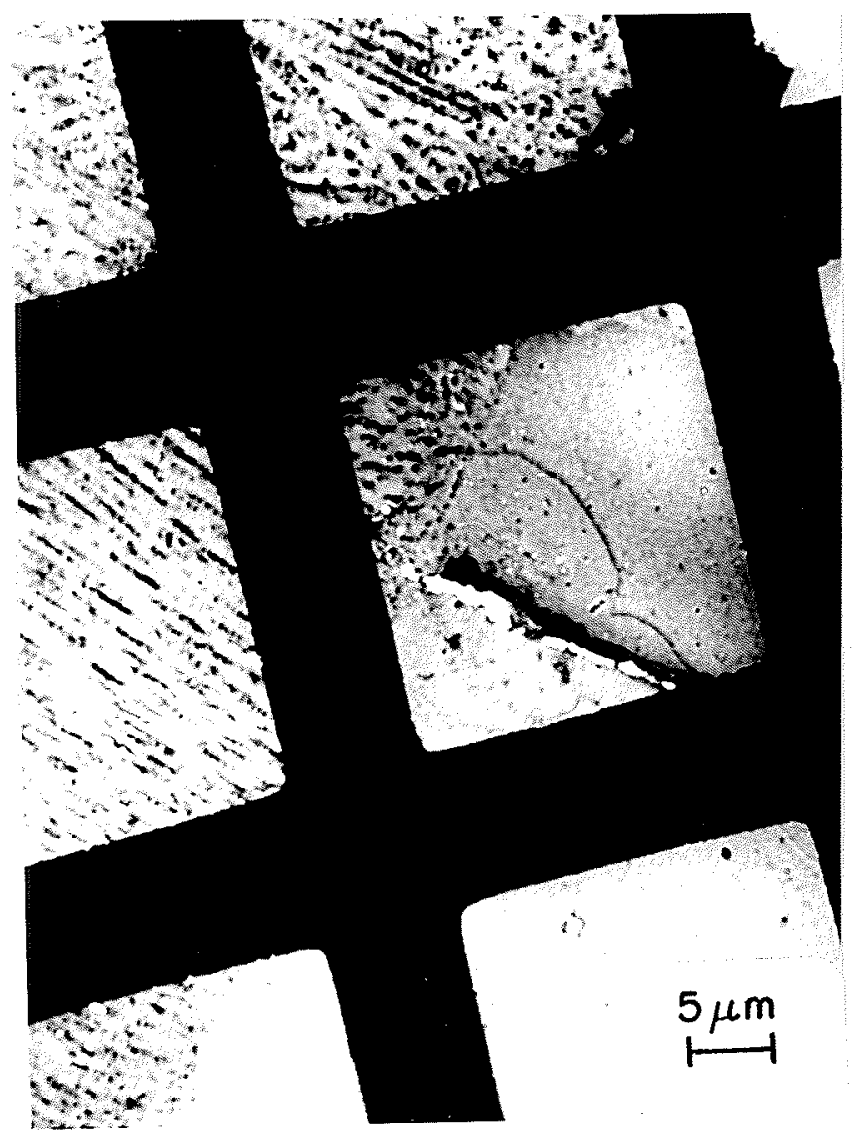

fig 4.14: Low magnification TEM micrograph of the fusion zone and HAZ extracted on a carbon replica. 




fig 4.15(a-c): TEM micrographs of a HAZ microfissure. Figs $4.15(b, c)$ show different areas of fig $4.15(a)$ at a higher magnification. 


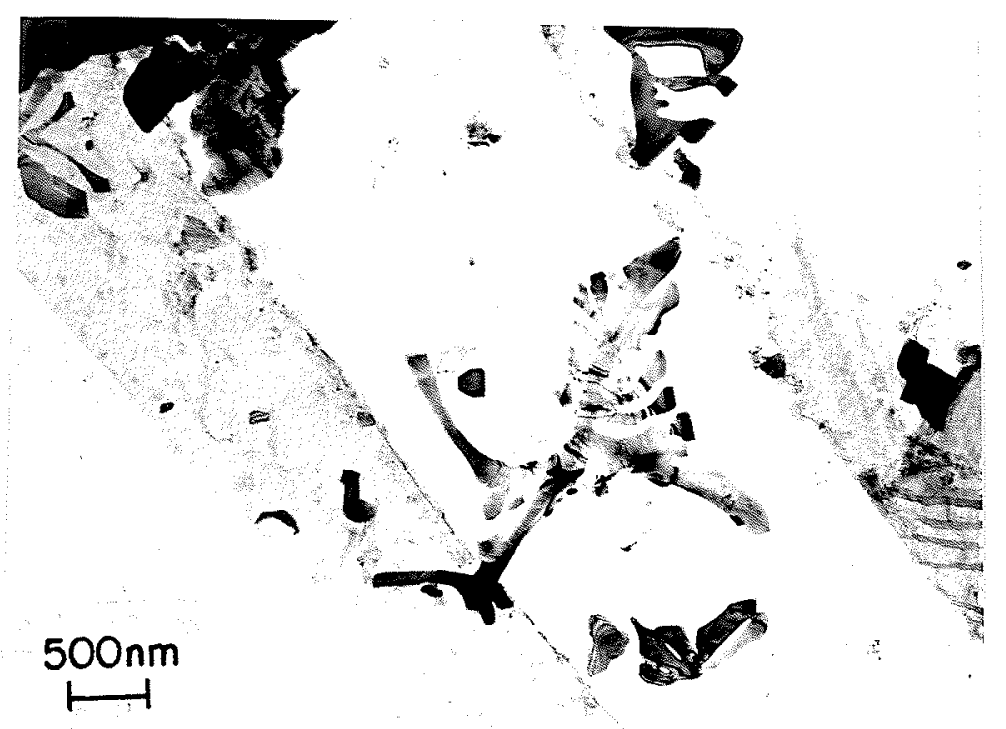

fig 4.15(d): TEM micrograph showing an interdendritic carbide sheet in a HAZ microfissure.

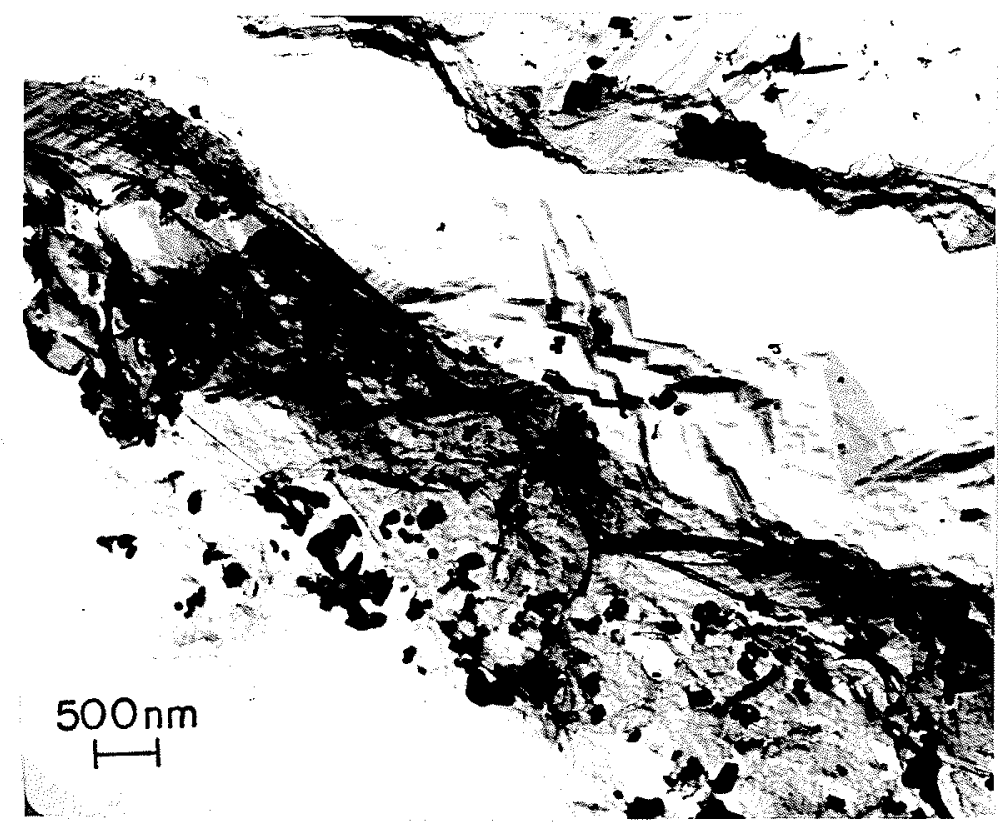

fig 4.15(e): TEM micrograph showing extensive fine carbide precipitation aside a HAZ microfissure. 


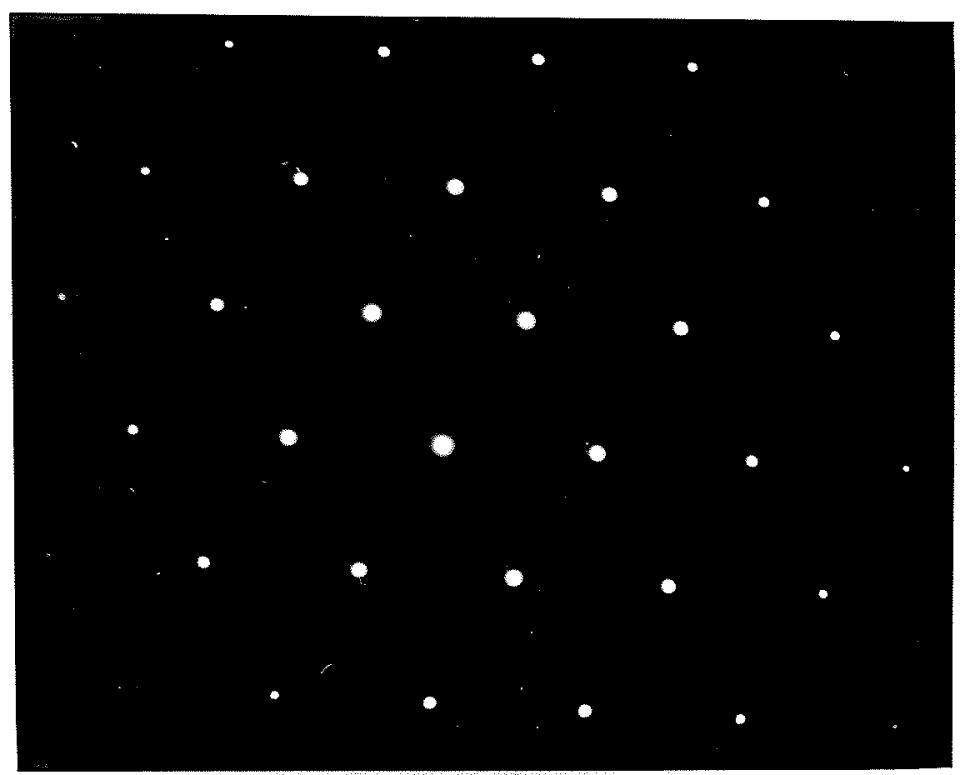

fig 4.16(a): A typical [0001] zone diffraction pattern obtained from an $\mathrm{M}_{4} \mathrm{C}_{2} \mathrm{~S}_{2}$ particle.

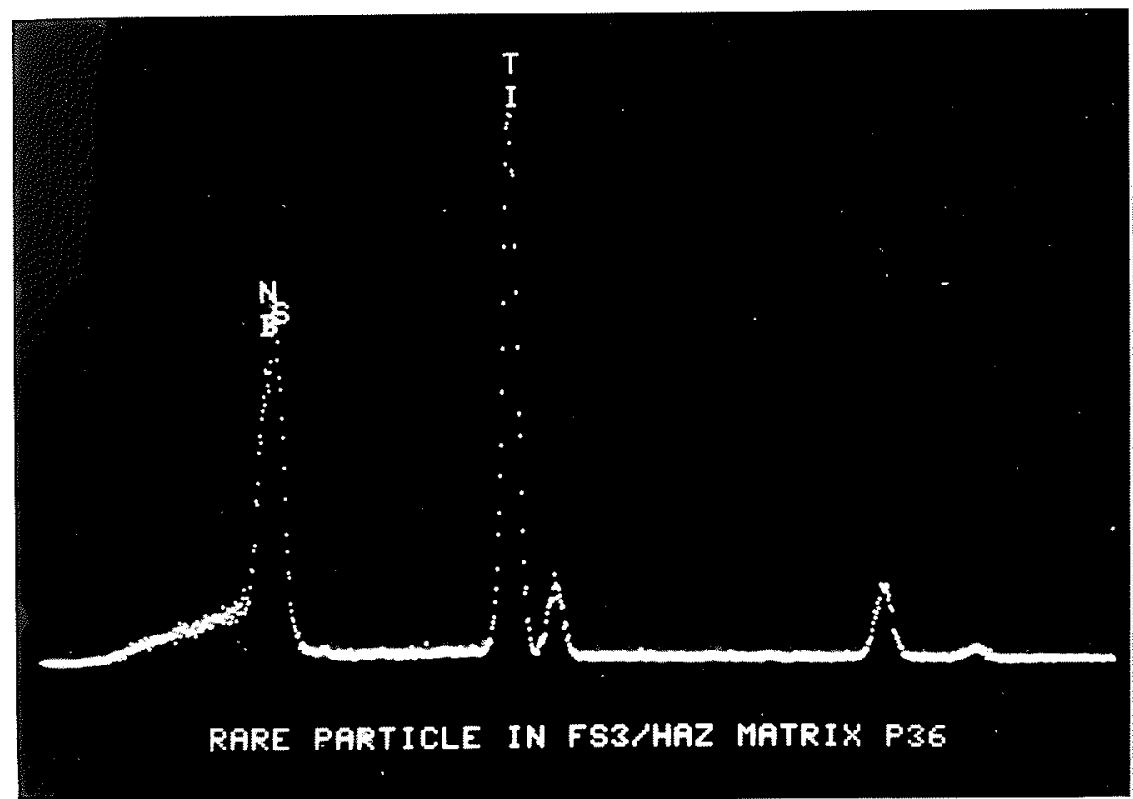

fig 4.16(b): A typical TEM/EDS spectrum obtained from an $M_{4} C_{2} S_{2}$ particle. 
obtained from this phase. $\mathrm{M}_{4} \mathrm{C}_{2} \mathrm{~S}_{2}$ hexagonal phase $(\mathrm{M}=\mathrm{Ti}, \mathrm{Nb}, \mathrm{Zr}$ ) has been reported to form in other superalloys viz., Astroloy, Pyromet*, 713 LC, Inconel 718, IN 10 and 979 [97]. The composition (metallic and S) of titanium carbosulphide in wt $\%$ as determined from TEM/EDS microanalyses is presented in table 4.9. It should be pointed out that the $\mathrm{Nb}$ and $\mathrm{S}$ peaks were not deconvoluted while quantifying the spectra.

The occurrence of MC carbides in three different morphologies can be easily appreciated if the formation of each type is associated with a different stage of the weld cooling cycle. The solidification pattern of the liquid on the microfissurred HAZ grain boundaries, as seen from the microstructures presented in the figures, is very similar to that observed in the fusion zone. The first solid to form from the liquid is the gamma dendrites followed subsequently by the gamma/carbide eutectic reaction. The carbides forming from the interdendritic liquid by the monovariant gamma/carbide eutectic reaction grows in form of sheets with arrow head and lobed morphologies with growth directions parallel to the cube axis. Continued formation of these carbides lead to the liquid film stage on the boundary and in the terminal stages of solidification these thin interdendritic films solidify as thin long continuous and/or semi-continuous film of carbides and some Laves phase. Since the MC carbides precipitated in solid state have been observed to have the regular cuboidal morphology, it is more likely that the fine cuboidal carbides that are observed along the $\mathrm{HAZ}$ boundaries are the ones that formed by the solid state precipitation process during the weld cooling cycle.

Table 4.9: Chemical composition (wt \%) of the trace phases

\begin{tabular}{llll} 
Phase & $\mathrm{Nb}$ & $\mathrm{Ti}$ & $\mathrm{S}$ \\
$\mathrm{M}_{4} \mathrm{C}_{2} \mathrm{~S}_{2}$ & 41.3 & 38.5 & 20.1 \\
\hline
\end{tabular}

* Pyromet is similar in composition to Incoloy 903 
Fig 4.17 shows a TEM micrograph of a warm worked HAZ grain boundary from a case $A$ weld made on forged and solution treated ring that resisted microfissuring. As can be seen in this figure, liquation is evident only at discrete pockets on the grain boundary. The solidification of these liquid pools was observed to follow the same sequence as the liquid in the fusion zone and in the grain boundaries that had developed microfissures. Interdendritic carbide sheets along with traces of Laves phase were observed. However, the liquid films were not extensive in their coverage of the grain boundary. Some local bowing of boundary segments at areas where liquation occurred can also be noticed in the figure. The lack of a continuous and/or semicontinuous liquid films appears to be the prime reason for these $\mathrm{HAZ}$ boundaries to have resisted microfissuring.

It should be noted that no MNP phosphides were observed in the fusion zone or in the HAZ microfissures. The same secondary phases that are observed in the fusion zone are observed in the HAZ microfissures except for the unidentified trace phase. However, MNP phosphides were observed to precipitate in clusters on $\mathrm{HAZ}$ grain boundaries lying farther away from the fusion zone. Fig 4.18 shows extensive MNP-phosphide precipitation on grain boundaries in the HAZ remote from the fusion line. The phosphides precipitated in the HAZ during the weld thermal cycle are easily differentiated from the pre-existing phosphides in the base metal owing to the lack of internal defects in them. As reported in the section on base metal microstructural characterization, copious precipitation of defect free phosphides was observed in Incoloy 903 isothermally aged for three hours at $850^{\circ} \mathrm{C}$.

An interesting aspect emerges from the study of the TEM/EDS spectra (figs $4.4(\mathrm{~g})$, $4.5,4.9,4.10,4.13(\mathrm{~b})$ and 4.16 (b)) obtained from the various phases observed in the base metal and in the HAZ microfissures. The EDS spectrum can be utilized as qualitative identification tool for the various intermetallic phases observed in Incoloy 903 because of the difference in the intensity (total counts) levels of the $\mathrm{Fe}, \mathrm{Ni}, \mathrm{Co}$ peaks. In MNP phosphides, the Co peak always shows more counts than the $\mathrm{Fe}$ and Ni peak counts, which are about the same. In $\delta$ phase the nickel peak is the most intense coupled with a low $\mathrm{Fe}$ 


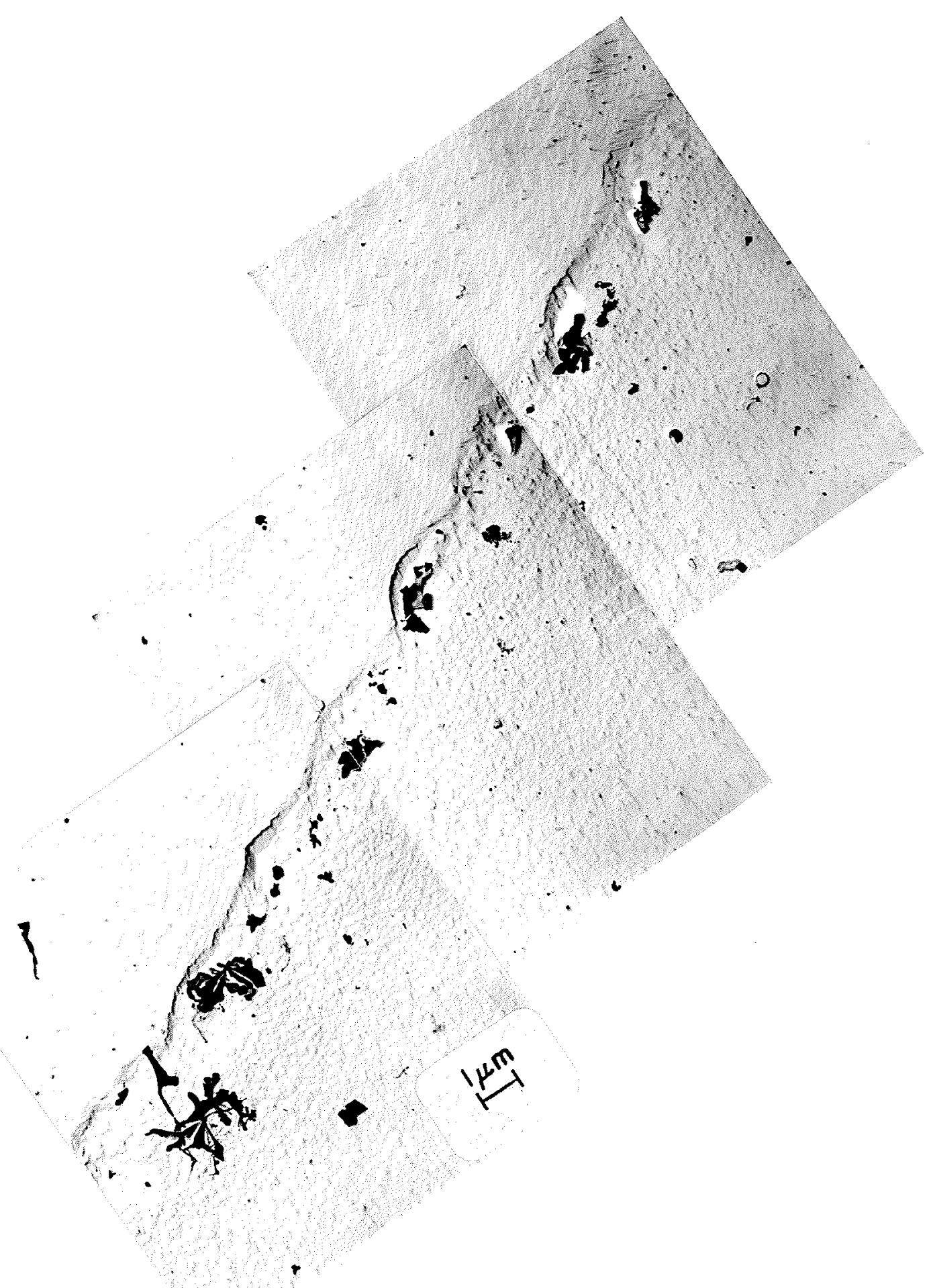

fig 4.17(a,b): TEM micrograph of a grain boundary in HAZ that resisted microfissuring. 


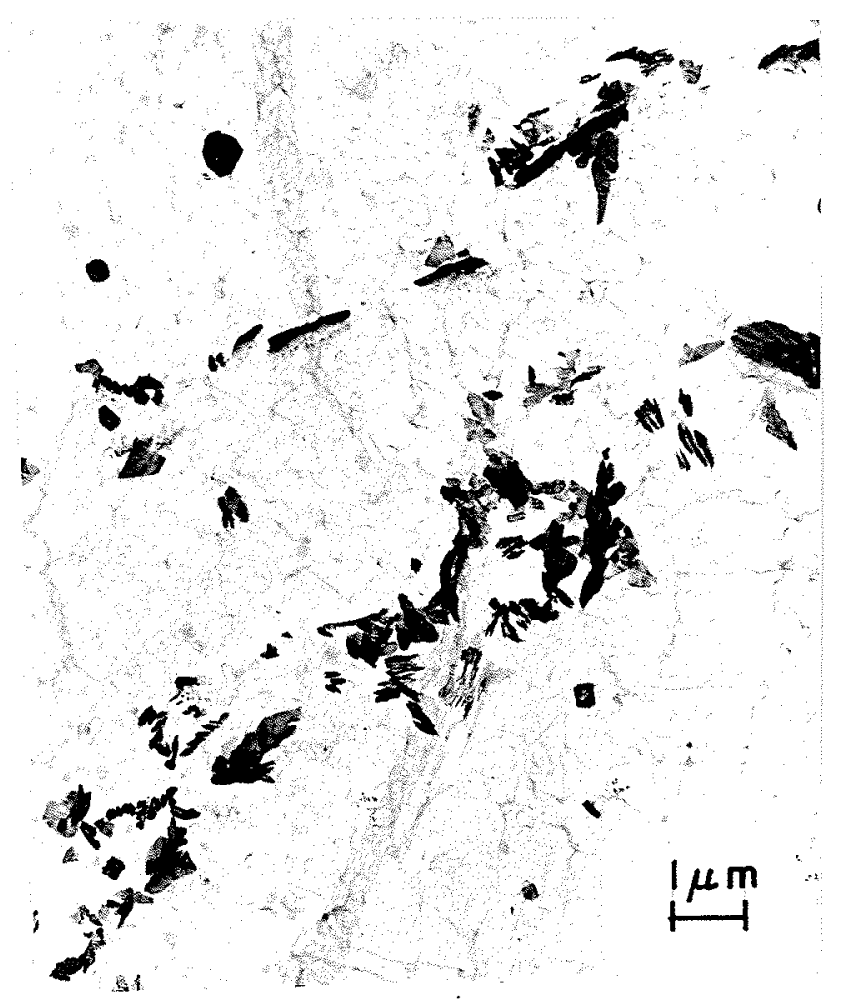

fig 4.18: TEM micrograph illustrating defect free MNP-phosphide precipitation in the far HAZ. 
peak count. In the Laves phase, while the nickel peak counts are again more than the $\mathrm{Fe}$ and $\mathrm{Co}$ peak counts, there are substantial counts in $\mathrm{Fe}$ and $\mathrm{Co}$ peaks. In the unknown blocky phase, the $\mathrm{Nb}$ peak is the most intense one with counts of $\mathrm{Fe}>\mathrm{Co}>\mathrm{Ni}$.

\subsubsection{2: Other Microstructural Conditions:}

Figs 4.19(a,b) show TEM micrographs of HAZ grain boundaries in the as-forged alloy solution treated at $975^{\circ} \mathrm{C}$ for an hour. The absence of solidification constituents due to terminal eutectic reactions on the thickened boundaries can be noticed. It can also be observed that coarsening of some of the carbides as well as total liquation and resolidification via a terminal eutectic reaction (gamma/MC carbide) occurred at some pockets in the HAZ matrix. In HAZ matrix, partially liquated large carbides can also be observed. Since the overall size of the weld and $\mathrm{HAZ}$ of case A welds was small, it was not possible even after several attempts to obtain electron transparent areas from specific regions of interest. However, TEM studies by carbon extraction replica's were sufficient enough to prove the nonexistance of terminal interdendritic solidification constituents on liquated grain boundaries that relieved most of their supersaturation by grain boundary liquid film migration. Further extensive experimental evidence as to this aspect is provided in the next section dealing with the optical and SEM observations on HAZ microstructures of welds made on material with different microstructural conditions.

Figs $4.20(a, b)$ show electron micrographs of the terminal solidification constituents in $H A Z$ microfissures extracted on a carbon replica from bead on plate welds made under case $A$ conditions on forged and solution treated ring further solution treated for 8 hours at $1100^{\circ} \mathrm{C}$. The morphology of the phases and the phases observed in the microfissures are identical to the ones observed in the microfissures of the forged and solution treated ring welds. Figs $4.21(a, b, c)$ show the secondary solidification constituents extracted from another HAZ grain boundary of the same sample. One can again notice the similarity between this boundary that resisted microfissuring and the one described in the previous 

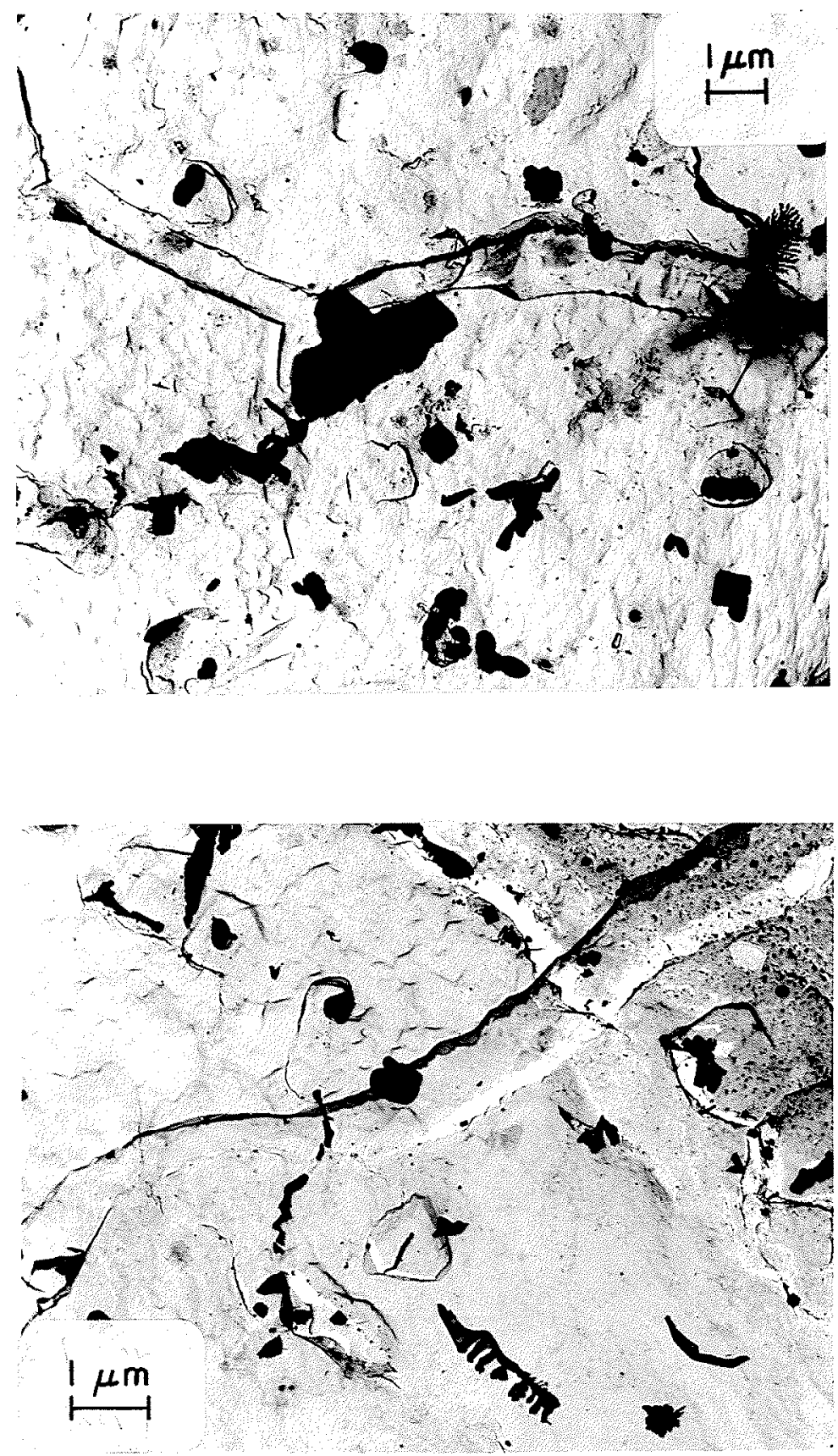

fig 4.19(a,b): TEM micrographs (on carbon extraction replica) of the thickened HAZ boundaries observed in case $A$ welds of as-forged samples solution treated at $975^{\circ} \mathrm{C}$ for an hour. 


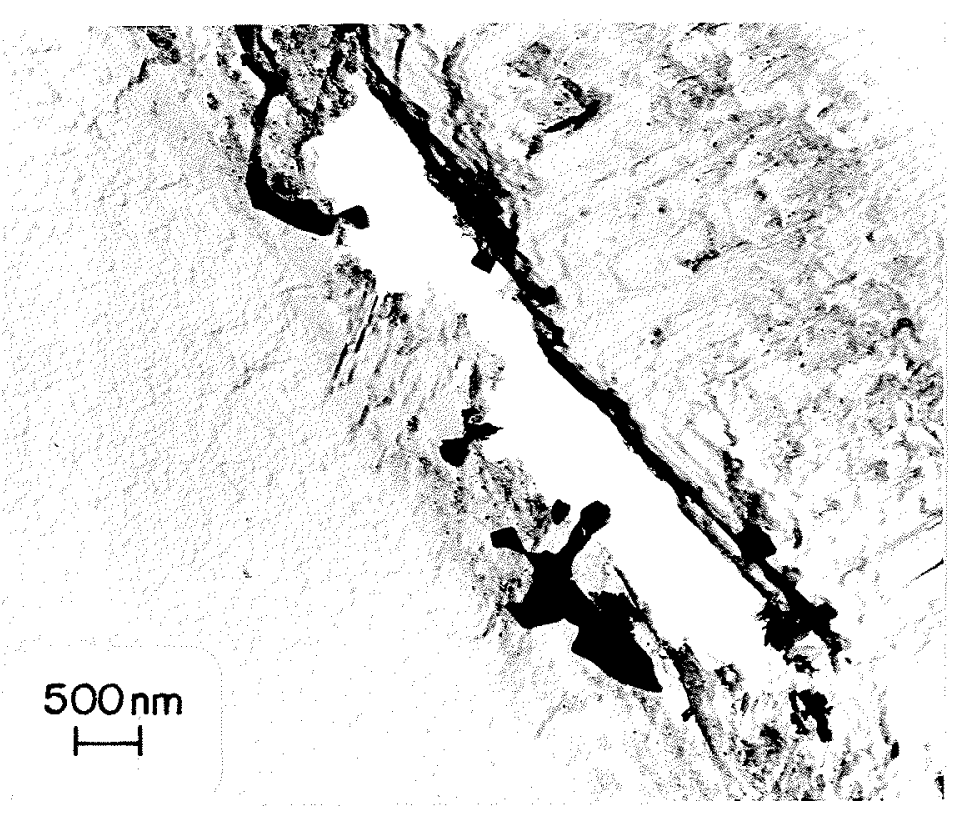

fig 4.20(a): TEM micrograph showing thin carbide and Laves phase sheets in case A weld $\mathrm{HAZ}$ of forged and solution treated ring further solution treated for $8 \mathrm{~h}$ at $1100^{\circ} \mathrm{C}$.

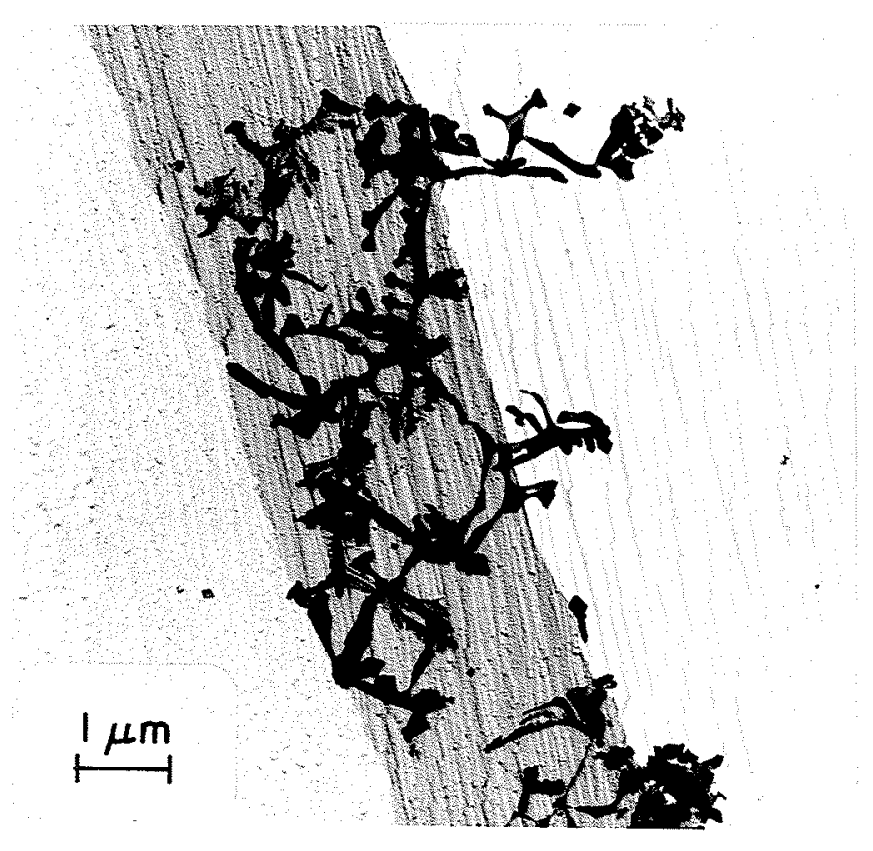

fig 4.20(b): TEM micrograph of a different area of the same microfissure as in 4.20(a) illustrating interdendritic carbides. 

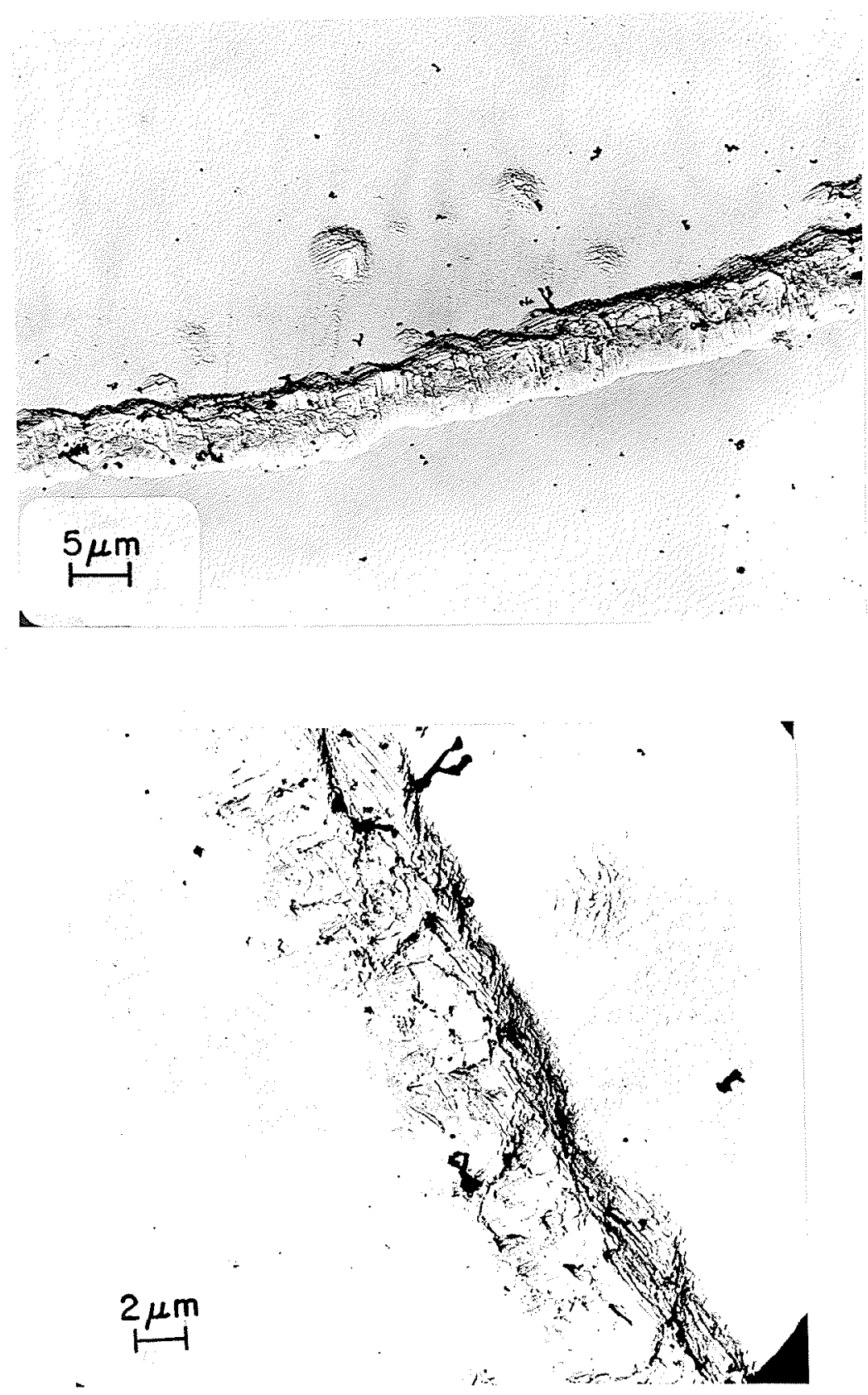

fig 4.21(a,b,c): TEM micrographs of a different HAZ grain boundary from the same replica shown in fig 4.20(a). Note the lack of continuous/semicontinuous sheets of terminal solidification constituents on this boundary that resisted microfissuring. 
section, the essential features being: (a) the terminal interdendritic liquid film is not extensive in the coverage of the grain boundary and (b) occurrence of some amount of liquid film migration that can be observed from the thickness of the boundary. Hence, it appears that the existance of a continuous/semicontinuous interdendritic liquid film is a necessary condition for the occurrence of $\mathrm{HAZ}$ microfissuring. The experimental evidence suggests the occurrence of HAZ microfissuring by the same mechanism(s) in the case of Incoloy 903 given both low and high temperature solution treatments.

\subsection{3: HAZ microstructure: Optical and SEM observations:}

Figs 4.22(a-e) show optical micrographs of the weld and HAZ of case A welds made on material in various microstructural conditions. Examination of these welds by optical microscopy revealed a narrow cast weld zone extending through approximately $90 \%$ of the thickness. This is commonly known as the spike. The remaining portion of the weld has a gradually increasing weld width and is known as the nail head. It can be observed that microfissuring generally occurs primarily in an area located at the junction of the nail head and the spike portions. Extensive HAZ microfissuring can be observed in the forged and solution treated ring, fig 4.22(a), as well as in the samples solution treated for $1 / 2$ $\mathrm{h}$, fig $4.22(\mathrm{~b})$, and $8 \mathrm{~h}$ at $1100^{\circ} \mathrm{C}$, fig $4.22(\mathrm{c})^{*}$. HAZ microfissuring was observed to a limited extent in the as-forged sample when the warm worked grain boundaries lie mostly parallel to the fusion line, fig 4.22(d). Minimal HAZ microfissuring was observed in the electron beam welds made on as forged samples solution treated for an hour at $975^{\circ} \mathrm{C}$,

* Since, all the samples solution treated at $1100^{\circ} \mathrm{C}$ in the range $1 / 2$ to 8 hours exhibited extensive HAZ microfissuring, no effort was expended in quantifying the data in terms of either total crack length or total number of cracks or average crack length. The $\mathrm{TCL}$ appeared to increase with increase in grain size at $1100^{\circ} \mathrm{C}$. However, the mean grain size of the samples solution treated at $975^{\circ} \mathrm{C}$ for one hour and $1100^{\circ} \mathrm{C}$ for half hour do not differ much from one another. Further details are provided in a later section. 
fig $4.22(\mathrm{e})$.

Fig 4.23(a) shows a SEM/BSE image of a microfissure observed in the HAZ and extending into the fusion zone. Fig 4.23(b) shows a higher magnification SEM/BSE image of a microfissure. A solidification microstructure similar to that of the fusion zone microstructure is evident in the figure. A careful observation of the figure reveals the presence of the typical arrow head and lobed morphology that is characteristic of the interdendritic MC carbides. SEM $x$-ray images and line scans indicated the microfissures to be enriched in niobium. Fig 4.23(c) shows a niobium x-ray image of a HAZ microfissure which clearly demonstrates niobium enrichment in the microfissures. These results confirm the formation of a niobium-enriched liquid phase on the HAZ boundaries by liquation and subsequent resolidification.

In optical and SEM micrographs of electropolished samples, the grain boundaries in the HAZ showed enhanced contrast. The enhanced contrast is observed due to the liquation occurring on the $\mathrm{HAZ}$ grain boundaries during the weld heating cycle. It may also be likely that, to an extent, the enhanced contrast may be due to the segregation of certain elements to the grain boundaries. Liquation or segregation of elements, as will be discussed later, significantly increase the HAZ microfissuring susceptibility. The boundaries that show HAZ microfissuring are delineated by an undulating or pleated band. This morphology is obtained because of the formation of gamma dendrites and secondary solidification constituents from the liquid present on the boundary. It was also observed that the solidification constituents form mostly on one side of the microfissure. This confirms that in the terminal liquid film stages, microfissuring generally occurs by decohesion across one of the solid-liquid interfaces.

The grain boundaries in HAZ that did not crack, in particular, the fine recrystallized grains, had a uniform thickened appearence, fig 4.24, as opposed to boundaries that show HAZ microfissuring which are delineated by an undulating or pleated band, fig 4.25(ac). This true morphology of the HAZ microfissures is lost, if the sample is etched or 
electropolished by etchants that attack the solidification constituents, in the present case, gamma and interdendritic MC carbides, in the microfissures. Only a careful choice of electropolishing agent reveals the true morphology of the microfissures. It has been shown in the earlier sections that the solidification sequence observed on HAZ microfissures is similar to the solidification sequence observed in the fusion zone. The pleated morphology is obtained because of the solidification of the liquid present on the boundaries into gamma dendrites and interdendritic solidification constituents.

Extensive HAZ microfissuring was observed on the large warm worked grain boundaries lying perpendicular to the fusion line. A partially liquated $M C$ carbide adjacent to the fusion zone can be observed in fig 4.26. As described in optical and SEM micrographs of electropolished samples, the grain boundaries in the HAZ show enhanced contrast due to the liquation occurring on the $\mathrm{HAZ}$ grain boundaries during the weld heating cycle. The liquid on the $\mathrm{HAZ}$ grain boundaries originates from the constitutional liquation of primary MC carbides, fine MC carbides and MNP phosphides present on the grain boundaries. It is possible that a part of the liquid in the HAZ close to the fusion line might have originated from back filling of the interdendritic liquid in the fusion zone along contiguous grain boundaries in HAZ due to capillarity effects. However, it will be shown in the discussion later, that significant backfilling of interdendritic liquid cannot occur.

The interesting observations in the present investigation is that $\mathrm{HAZ}$ microfissuring is observed on the warm worked boundaries in the forged and solution treated ring as well as on the recrystallized grains in the forged and solution treated ring further solution treated for $1 / 2 \mathrm{~h}$ at $1100^{\circ} \mathrm{C}$. Minimal HAZ microfissuring was observed on the fine recrystallized grains forming the necklace in the forged and solution treated ring. In contrast to the pleated morphology of the $H A Z$ grain boundary microfissures, the fine recrystallized grains that did not crack,fig 4.27(a-e), had a uniform thickened appearence and showed no evidence of normal dendritic solidification constituents. The same phenomenon is observed on the HAZ grain boundaries of the as-forged alloy solution treated at $975^{\circ} \mathrm{C}$. The thick- 


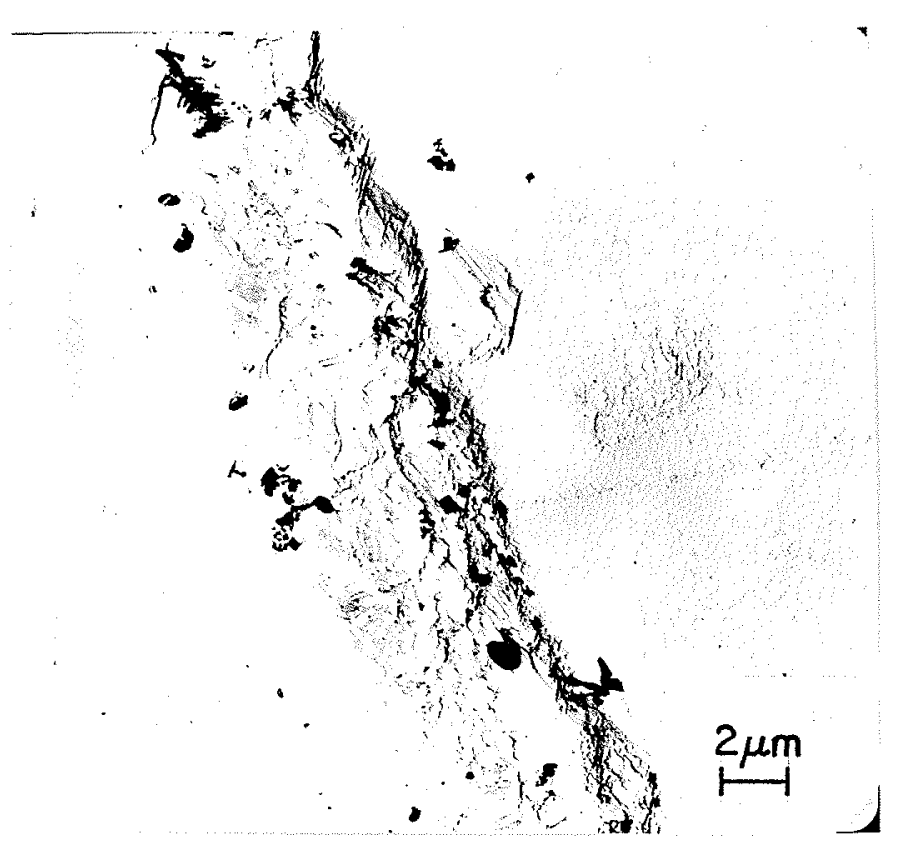

fig $4.21(c)$

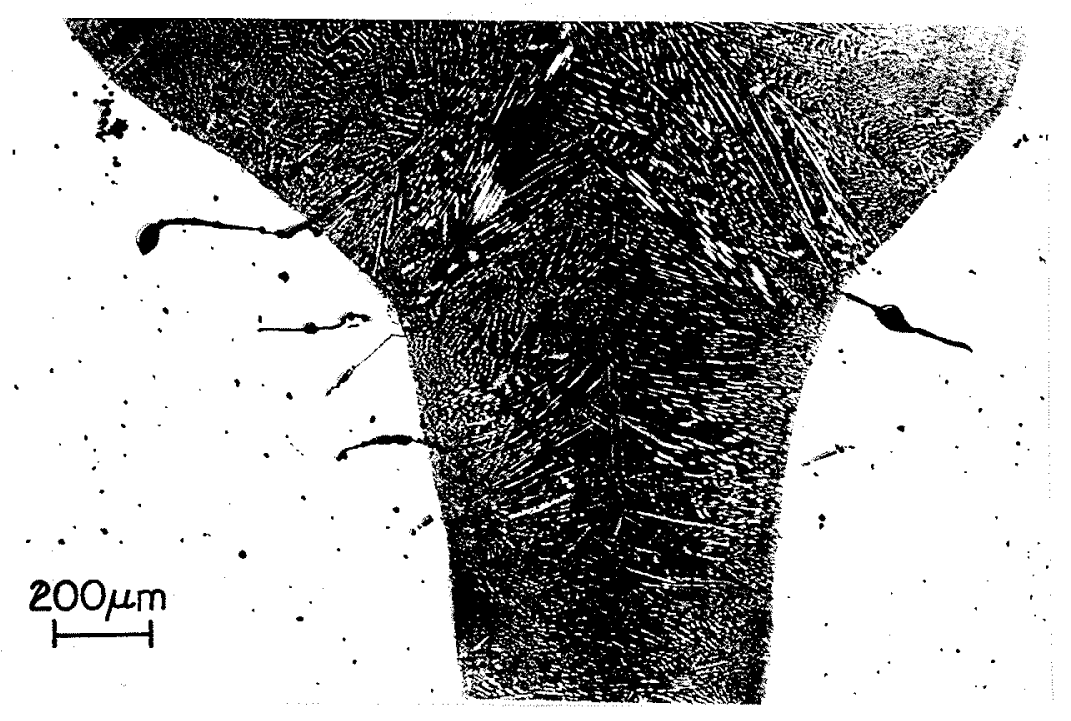

fig 4.22(a): Optical micrograph of case A welds (fusion zone and HAZ) of forged and solution treated ring. 

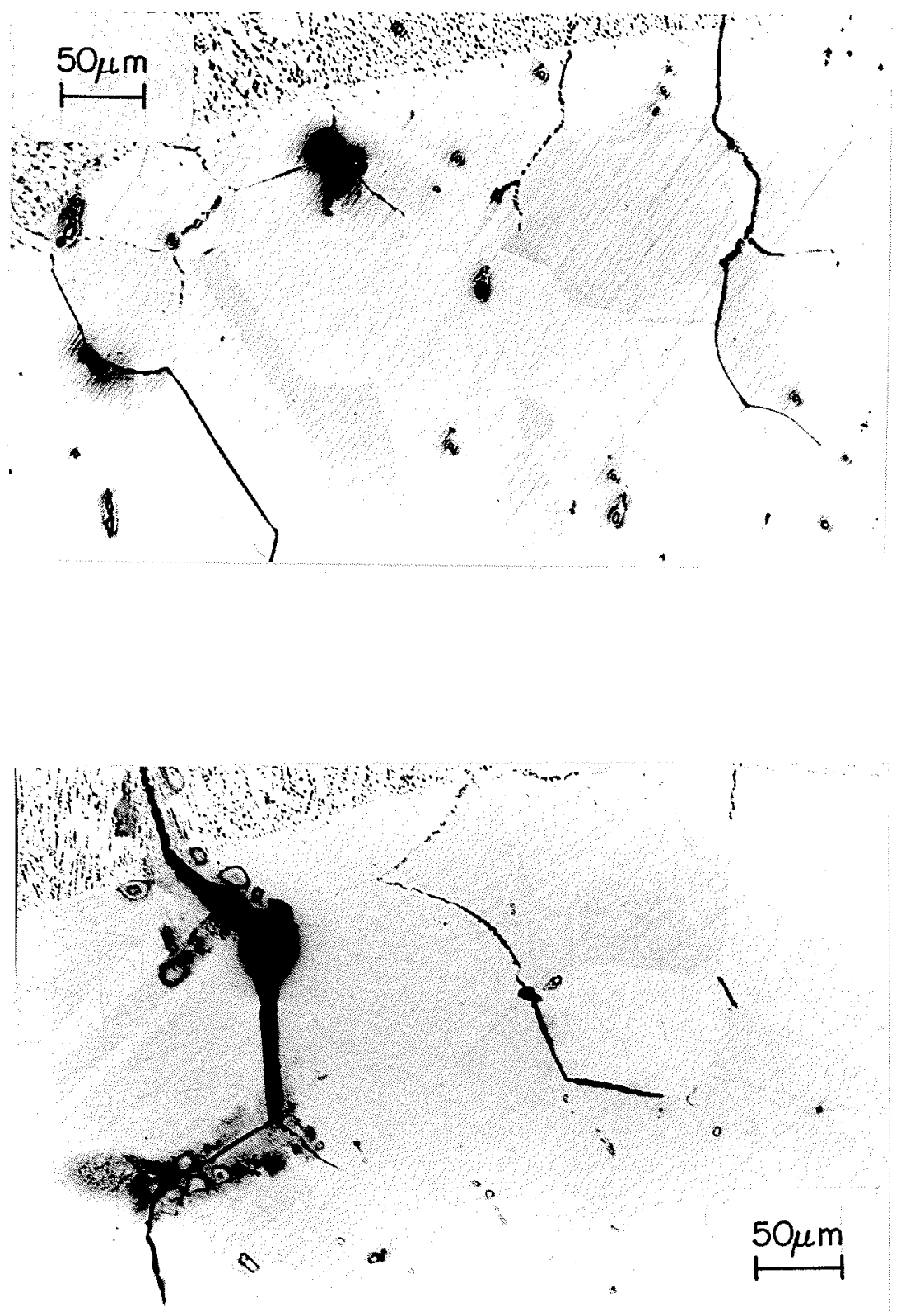

fig 4.22: Optical micrograph of case A welds (fusion zone and HAZ) of forged and solution treated ring further solution treated for (b) $1 / 2 \mathrm{~h}$ at $1100^{\circ} \mathrm{C}$ and (c) $8 \mathrm{~h}$ at $1100^{\circ} \mathrm{C}$. 

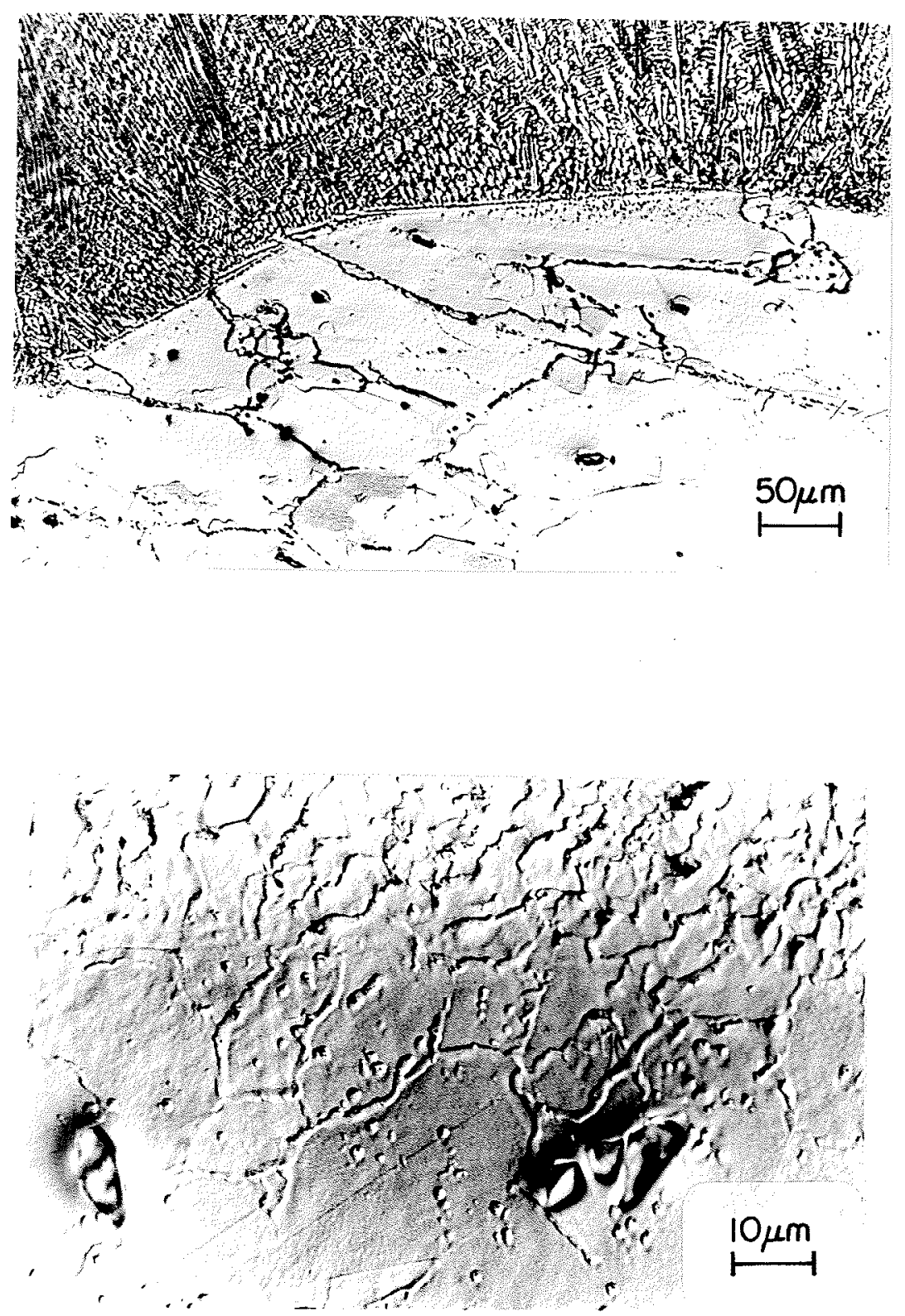

fig 4.22: Optical micrograph of case A welds (fusion zone and HAZ) of as-forged alloy (d) with warm worked boundaries lying nearly parallel to the fusion line and (e) solution treated for an hour at $975^{\circ} \mathrm{C}$. 


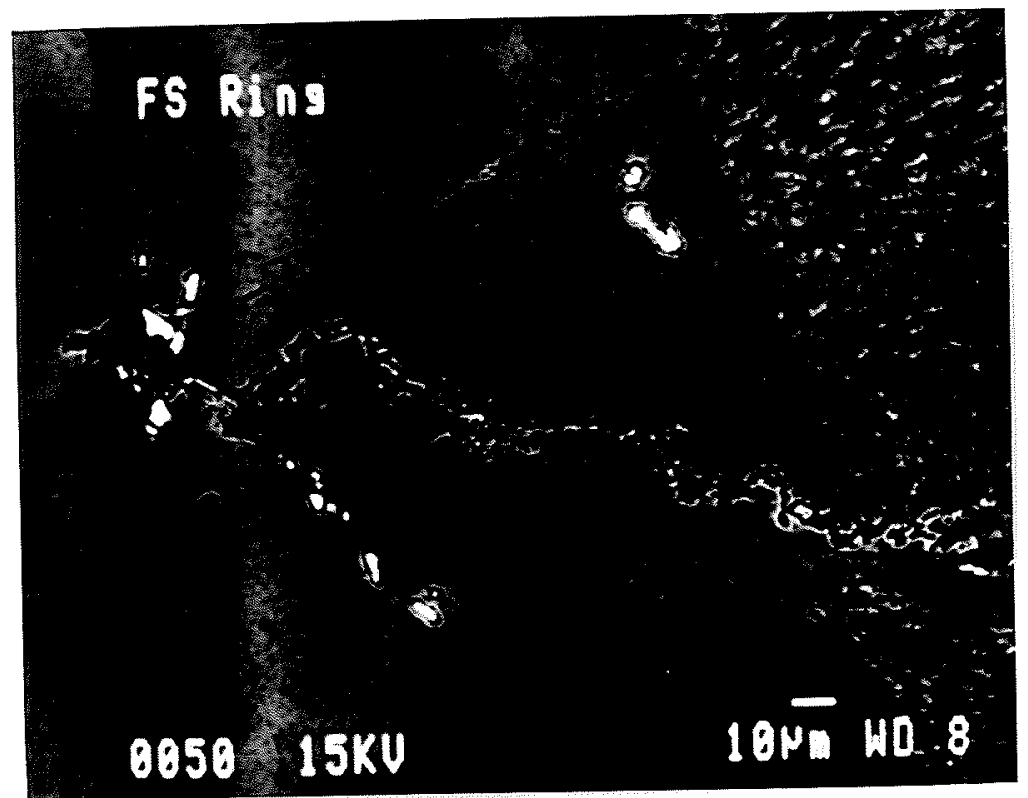

fig 4.23(a): SEM/BSE image of a microfissure in the HAZ.

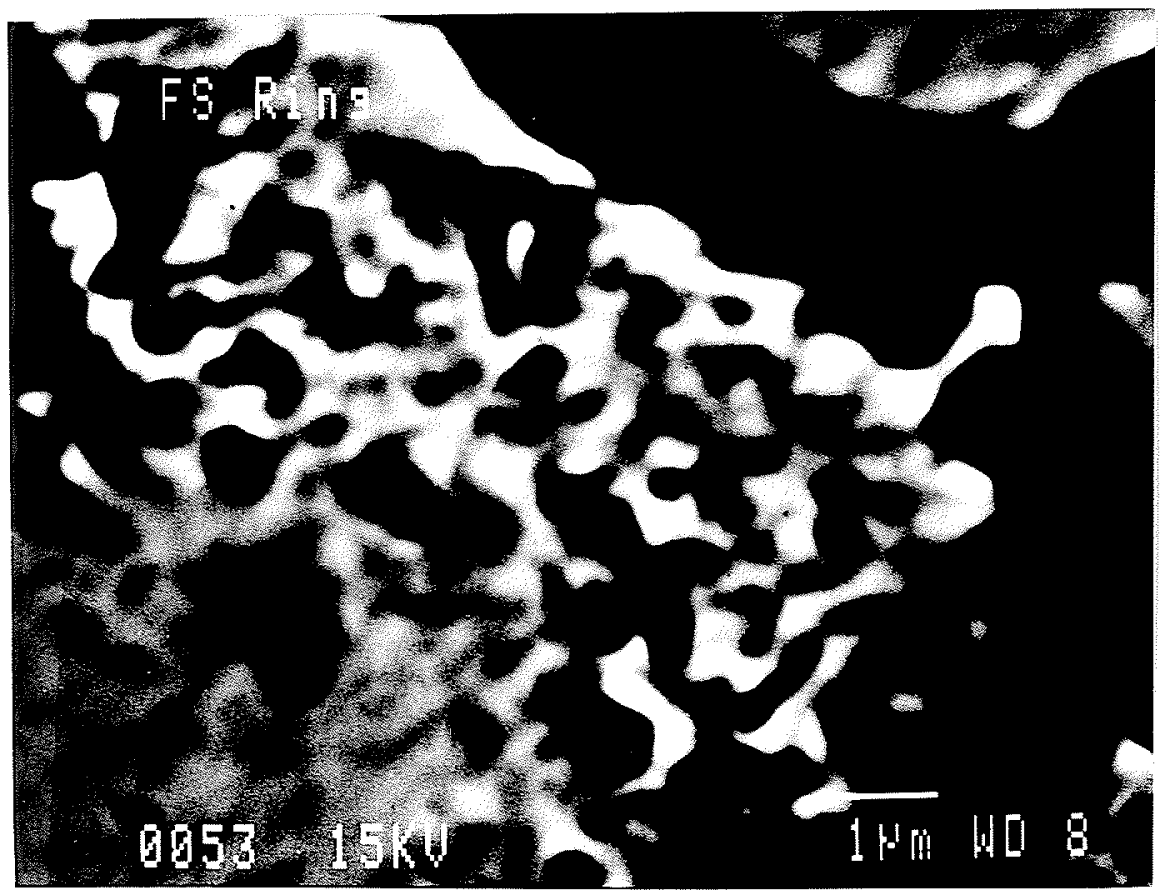

fig 4.23(b): SEM/BSE image illustrating interdendritic constituents observed in a microfissure. 


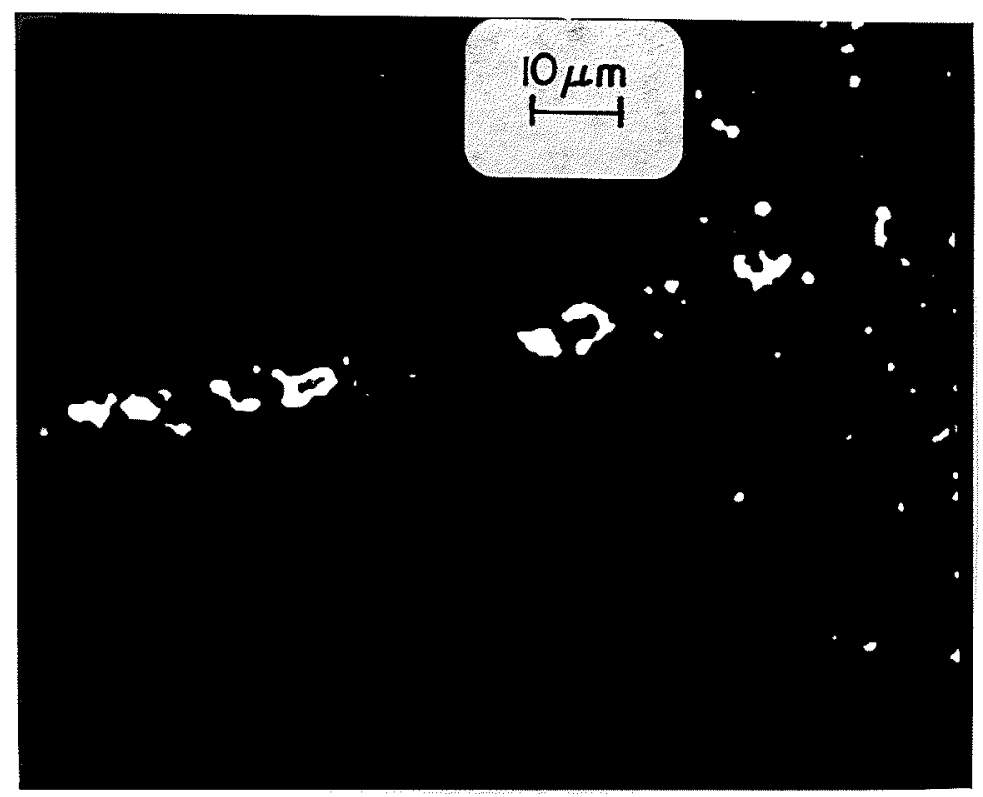

fig $4.23($ c): Niobium $x$-ray map of a HAZ microfissure.

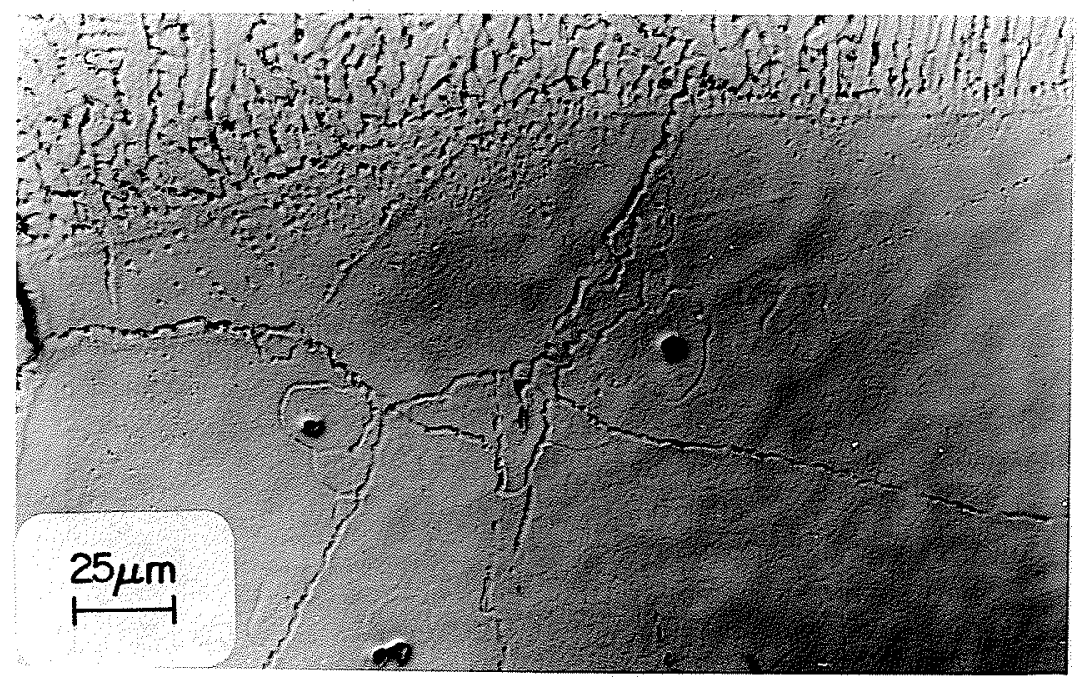

Fig 4.24: Optical micrograph showing the thickened appearence of the fine recrystallized grains. 



Fig 4.25(a-c): Optical micrographs illustrating the pleated morphology of HAZ microfissures. 




fig 4.26: SEM micrographs showing partial primary MC carbide liquation in HAZ.

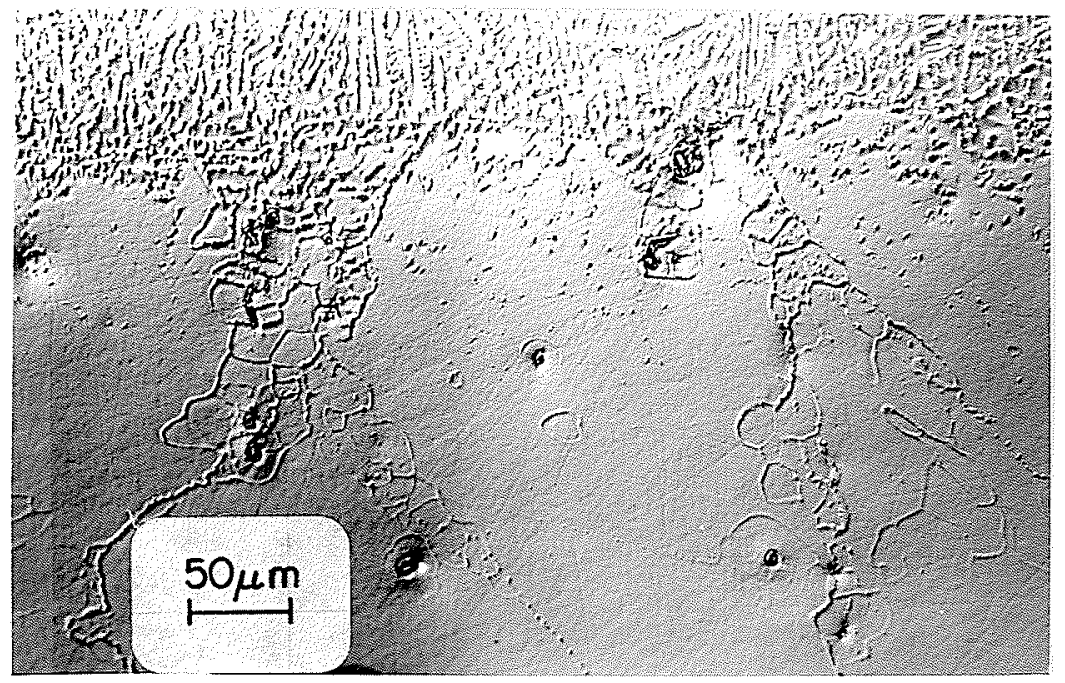

Fig 4.27(a): Optical micrograph showing the thickened appearence of the fine recrystallized grains. 



Fig $4.27(b, c)$ : SEM micrographs showing the thickened appearence of the fine recrystallized grains. 

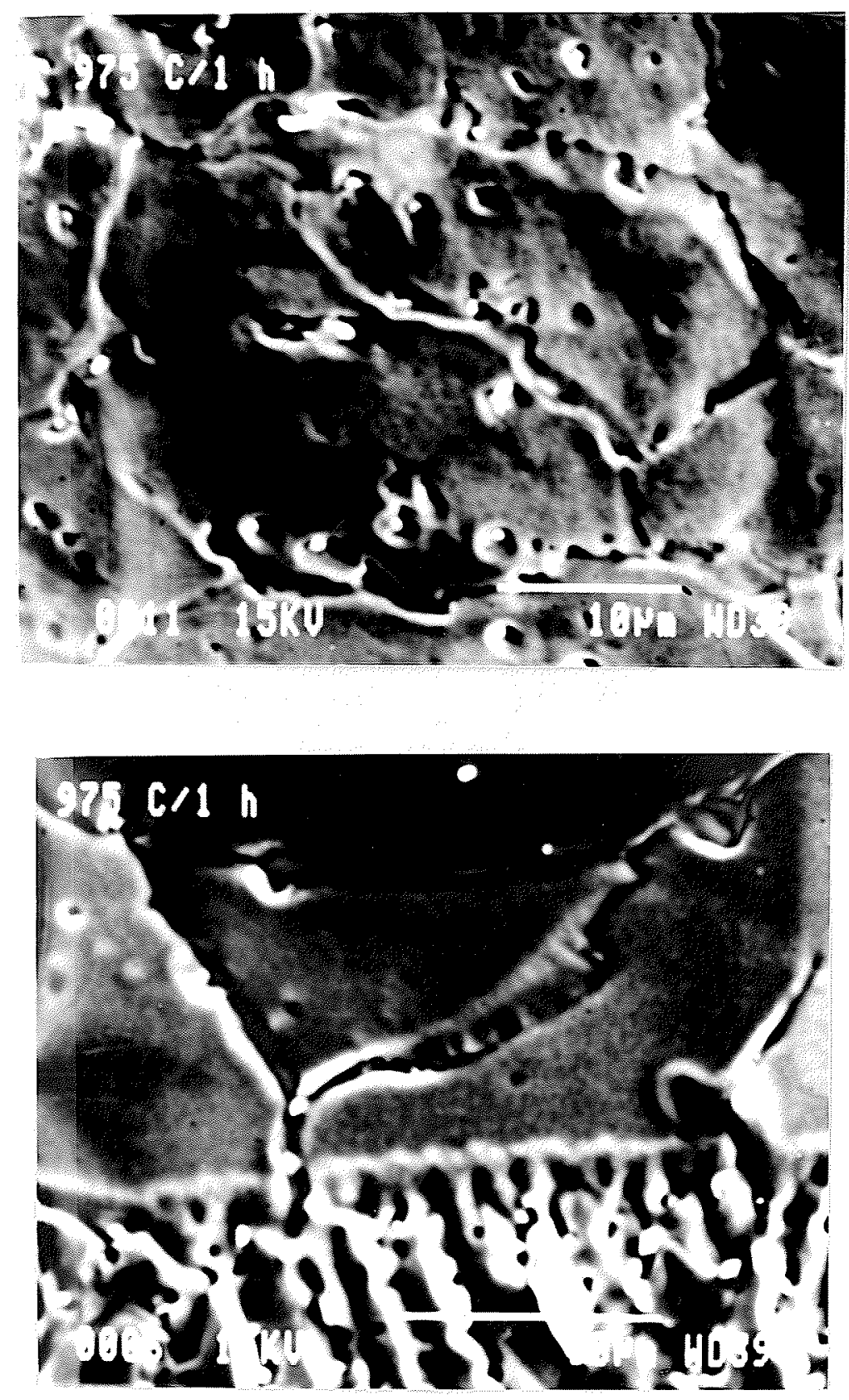

Fig 4.27(d,e): SEM micrographs showing the thickened appearence of the recrystallized grains observed in case $A$ welds of as-forged samples solution treated at $975^{\circ} \mathrm{C}$ for an hour. 
ened areas are formed due to the migration of grain boundary liquid film segments into the gamma matrix leaving behind an alloyed region. Grain boundary liquid film migration is an alternative process of equilibration of the metastable supersaturated liquid film. The newly formed alloyed regions (niobium-enriched gamma) due to liquid film migration (LFM), can be distinguished from the differential contrast between the freshly formed regions and the original matrix. This is because, the composition of the newly formed regions is the composition of the solid at equilibrium with the liquid. Grain boundary LFM was rarely observed on the large warm worked grain boundaries. Instead, the metastable liquid film solidifies in a normal manner leading to the formation of secondary terminal solidification constituents often incurring HAZ microfissuring and the formation of the characteristic pleated morphology.

In most cases, the liquid film migration on the fine recrystallized grains was uniform. However, sometimes the migration direction altered at different sections on some of the liquid films resulting in a zig-zag grain boundary pattern. Quantitative microanalysis by SEM/EDS (table 4.10) indicated that the migrated regions were enriched in niobium as compared with the bulk matrix. Hence, the migration is accompanied by the formation of a niobium enriched gamma solid solution behind the migrating liquid films. The difference in niobium content between the bulk and the newly formed regions is responsible for the differential contrast observed in the optical microscope and SEM. The increase in niobium content in the newly formed regions suggest constitutional liquation of niobiumrich carbides and phosphides present on the boundaries and formation of a metastable liquid film on the HAZ grain boundaries during the weld heating cycle and its subsequent migration by LFM to relieve the niobium supersaturation.

In summary, phase constituents of two kinds of morphology are observed on the liquated $H A Z$ grain boundaries;

(i) grain boundaries where the supersaturation in the metastable liquid is relieved by normal solidification leading to the formation of secondary terminal solidification constituents and 
often incurring $\mathrm{HAZ}$ microfissuring if continuous and/or semicontinuous interdendritic liquid films exist and

(ii) grain boundaries where the partial equilibration of the metastable liquid film completely occurred by LFM, or to be more precise, the supersaturation in the liquid film was eliminated to the extent required for the preclusion of the monovariant eutectic reaction, $\mathrm{L}+\gamma \rightarrow \mathrm{L}+\gamma+(\mathrm{Nb}, \mathrm{Ti}) \mathrm{C}$. 
Table 4.10: The chemical composition in wt \% of the LFM regions and the matrix as determined by SEM/EDS quantitative microanalyses.

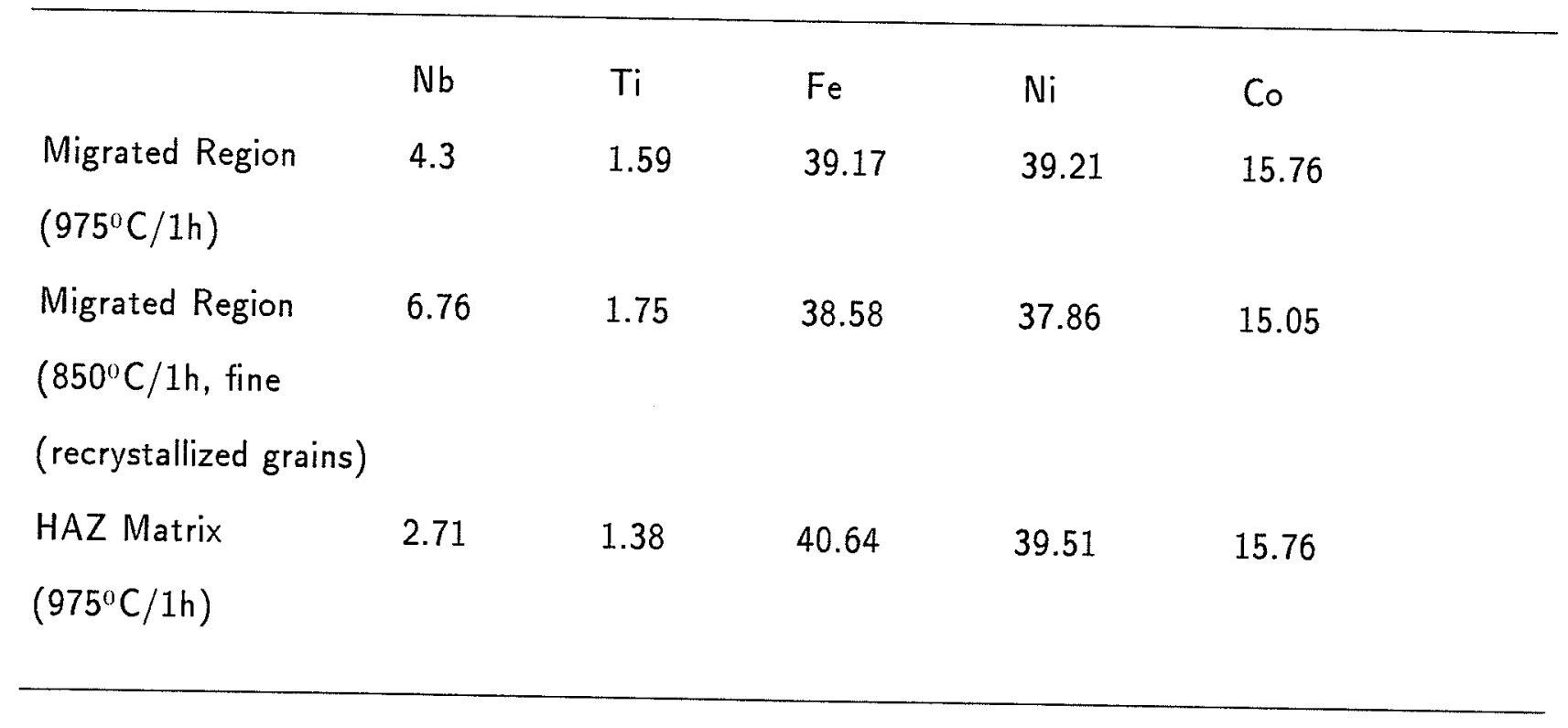




\section{4: GRAIN BOUNDARY LIQUID FILM MIGRATION}

The preceding section discussed the occurrence of grain boundary liquid film migration in the HAZ of Incoloy 903 weldments under certain conditions. The present section discusses the phenomenon, it's thermodynamics and kinetics. Subsequently, the experimental observations of the preceding section will be explained based on the theoretical analysis of LFM.

The migration of thin liquid films of one metal between grains of another, from one grain into the other leaving behind alloyed regions has been observed during many experiments on liquid phase assisted-sintering by Yoon and co-workers [118-125]. Following Hillert [126], they attributed the migration to the coherency strain developed in the grain being consumed by the migrating liquid film by volume diffusion of solutes into this grain. Liquid films between grains have been observed to migrate leaving behind alloyed or dealloyed zones when the equilibrium is upset either by changing the temperature $[119,123,125,127]$ or by changing the composition of the liquid phase $[120-123,125,127]$.

This process brings a system closer to a new equilibrium condition and is now referred to as liquid film migraion (LFM). It seems as an alternative to volume diffusion controlled processes as a mechanism for equilibration.

Though microstructural evidence for LFM in the HAZ during welding existed in the literature for a long time, the phenomenon itself has only been recently recognized and attributed to LFM. It was erroneously believed earlier that the thickened regions originated from the solidification of the back filled interdendritic liquid from the fusion zone. Owczarski et al. [1] have observed liquated grain boundary regions to migrate back and forth across a differentially etched band in Udimet 700 synthetic heat affected zone specimens. Voorhees et al. [128] have reported migration of liquified grain boundaries in autogeneous electron beam welds of $2024 \mathrm{Al}$ where the welding resulted in grain boundary migration velocities of $1 \mu \mathrm{m} / \mathrm{s}$ in the partially melted zone due to the large $\left(10^{6} \mathrm{~K} / \mathrm{min}\right)$ temperature gradients. Radhakrishnan and Thompson [129] have observed LFM in the 
HAZ of arc spot welds in Inconel 718. Reexamination of the results of Owczarski et al. [1] provide convincing evidence to the fact that the thickened regions form by the occurrence of LFM because, no fusion zone exists in synthetic HAZ specimens to provide any backfilled liquid.

Grain boundary liquid films form when the local alloy concentration significantly lowers the melting point at the grain boundaries. Grain boundaries may be infiltrated by a liquid layer considerably below the bulk melting temperature if wetting occurs. Alternately, the constitutional liquation of the precipitates on the grain boundaries due to rapid nonequilibrium heating, may lead to the formation of a liquid film. The nature of the driving force for the initiation of LFM (to be discussed later in this section) requires the grain boundaries to be in intimate contact with the liquid source, i.e there should exist two solid-liquid interfaces so that a diffusive flux can exist in the liquid film.

Since the lattice parameter is a function of composition, most compositional inhomogemeities will lead to a stress field in the latice. The lattice diffusion of a solute from the interface into a surface layer can, therefore, cause such a stress field. When the diffusion layer is thin compared to the principal radii of curvature of the surface of the solid, the stress depends only on the difference between the composition produced by lattice diffusion, $\left(\bar{C}_{S}-C_{0}\right)\left[\bar{C}_{S}\right.$ is the composition of the strained layer and $C_{0}$ is the bulk composition] and, for elastic anisotropy, on the surface normal, $\hat{n}$. Consider the case of two grains separated by a liquid film. If the stresses in these adjacent grains are different such that the liquids in equilibrium with these grains have different compositions, a concentration gradient will exist in the liquid film and results in net atomic motion and liquid film migration.

Considerable experimental evidence exists $[119,121,122,125,130]$ for the coherency strain energy developed in the thin diffusion layer in the grain being consumed during LFM providing the driving force for the reactions. Hillert [126] has provided a model for a direct driving force which depends upon the strain energy built up in a thin region 
of one crystal adjacent to the liquid film when solute diffuses a small distance away from the boundary to form an alloyed layer. It is presumed that volume diffusion is not negligible and there exists some misfit between solute and the solvent so that the volume of the alloyed layer is altered but remains constrained by the large bulk of the unalloyed material. The strain which is required in the alloyed layer in order to maintain coherency then provides a local increase in free energy, the effects of which are visualized using a free energy-composition diagram shown in fig 4.28 , which refers to the local equilibria at the solid/liquid interfaces bounding a thin film of liquid.

It is assumed that on the introduction of the liquid film which has a different composition than the solid crystals, local thermodynamic equilibrium is rapidly set up by means of dissolution of solid in the liquid and or adsorption of atoms from the liquid on the solid surfaces. At this point, the equilibrium compositions at the surfaces and in the liquid are found from a straight forward common tangent construction between the free energy curves for the solid and liquid phases. These compositions are now $C_{S}$ and $C_{L}$, respectively. Now consider just one of the solid/liquid interfaces. If solute diffuses a small distance into the solid, a strained coherent layer will be created. A thin layer of a different composition which is coherent with the grain of composition $C_{0}$ will have an extra energy due to coherency, $W$, given by:

$$
W=\frac{E V_{m} \eta^{2}}{(1-\nu)}\left(C_{0}-C_{X}\right)^{2}
$$

where $E$ is the Young's modulus of elasticity, $\nu$ is the Poisson's ratio, $\eta$ is the atomic misfit parameter. The Gibbs free energy curve for the coherent layer is thus tangent to the normal curve at $C_{X}=C_{0}$. With the addition of the influence of the elastic strain energy in the solid, a new equilibrium condition is set up, as defined by the common tangent to the free energy curves of the strained solid and the liquid, as indicated in fig 4.28. Thus the liquid composition in equilibrium with the strained solid is slightly 


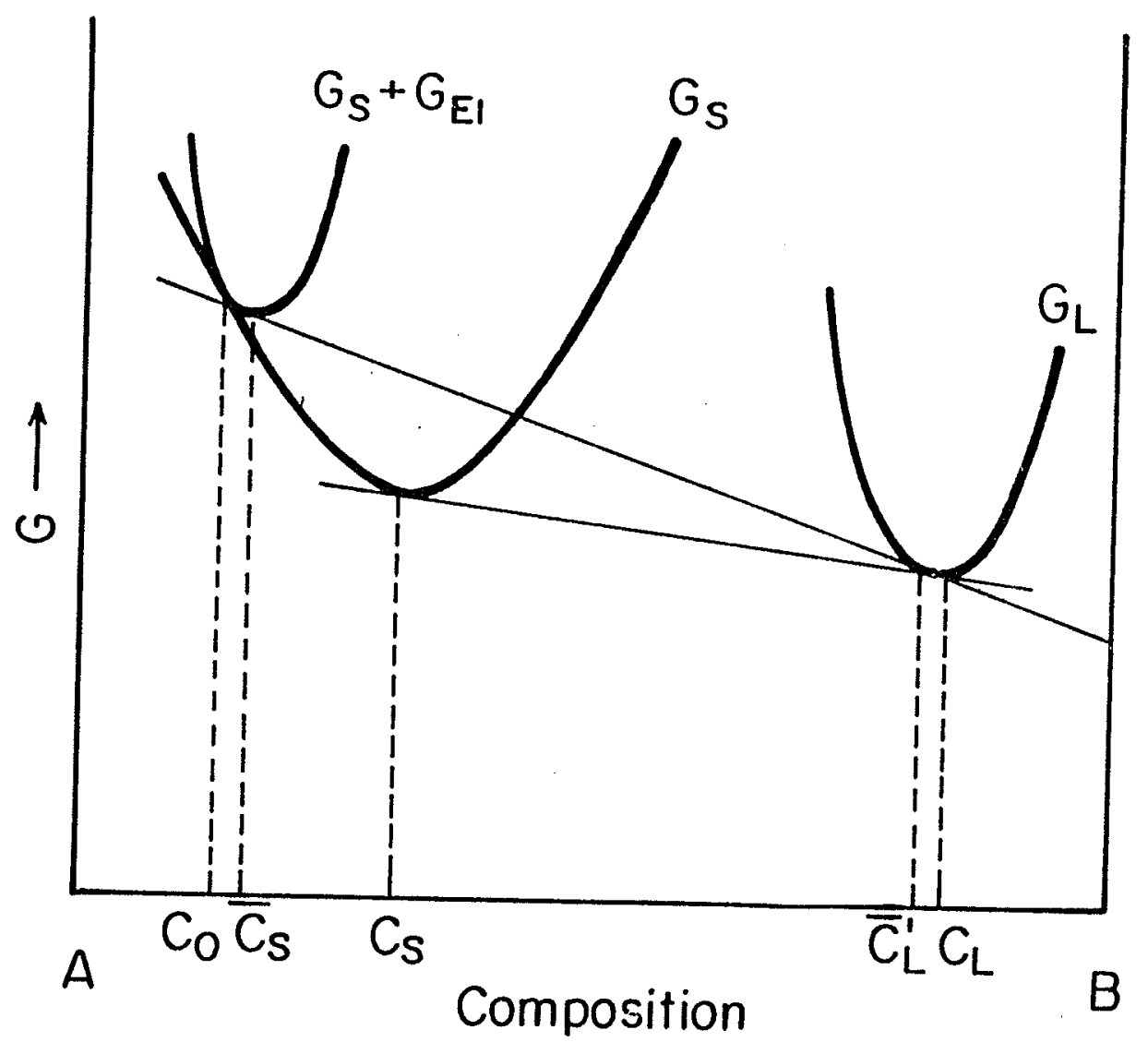

Fig. 4.28: Free energy curves for solid and liquid phases in a two component ( $A$ and $B$ ) system : Curve marked $G_{L}$ applies to the liquid phose while the curve marked $G_{S}$ applies to the unstresse solid phase : the usual tangent construction gives the composition of the solid and liquid in contact with each other at equilibrium at $\mathrm{C}_{S}$ and $\mathrm{C}_{L}$ respectively. For a solid with initial composition $\mathrm{C}_{0}$, diffusion of solute into or out of the surface forms an elastically stressed layer, the free energy of which is increased by on amount $G_{E} l$, which varies as the square of the difference in concentration from $\mathrm{C}_{0}$. In view of this stressed layer, a new equilibrium composition of $\bar{C}_{S}$ and $\bar{C}_{L}$ are established. 
shifted from that in equilibrium with the unstrained solid. The new compositions are $\bar{C}_{S}$ and $\bar{C}_{L}$. Suppose that an incoherent nucleus forms. It would then take a compostion of $C_{S}$ and, since its tangent falls below the other tangent, it could draw material from the coherent layer. The driving force per mole of atoms which are thus taken from the initial material, may be estimated as $\Delta G_{d}^{\alpha}-\Delta G_{d}^{E l}$. This may be regarded as the driving force for the nucleation of an incoherent nucleus as well. Once the nucleation has occurred, the nucleus may continue to grow due to the same driving force. Alternately, if the rate of migration is high enough, $D_{S} / v$ may become so small that the compositional discontinuity increases further and the driving force gets larger. Here, $D_{S}$ is the lattice diffusivity and $v$ is the velocity of migration. It is evident that a larger value of misfit parameter should lead to a very steep Gibbs energy curve for the coherent layer. It would thus have a composition very close to $C_{S}$ and though the actual coherency strain energy would be small, the driving force would be high. Assuming ideal solution behavior, Hillert [126] estimates the magnitude of the driving force as,

$$
\Delta G_{A}=\frac{E V_{m} \eta^{2}}{(1-\nu)}\left(C_{S}-C_{0}\right)^{2}
$$

when the misfit effect is low and $\bar{C}_{S}$ is close to $C_{S}$ rather than $C_{0}$. As mentioned earlier, large $\eta$ value result in $\bar{C}_{S}$ being closer to $C_{0}$ rather than $C_{S}$, a case where the driving force will be large but the actual coherency energy would be low.

Now, if a similar process occurs at the other liquid/solid interface, but with a different extent of diffusion or with a different elastic modulus corresponding to a different orientation, then the liquid composition in equilibrium with the second interface will be different from that in equilibrium with the first. A diffusive flux across the liquid layer will be established and this will carry material from the more highly stressed solid to the less highly stressed solid causing the growth of the less highly stressed solid at the expense of the more highly stressed solid and driving the migration of the liquid film. A feature of the 
model is that the composition of the growing grain is the solid composition at equilibrium with the liquid.

The initiation and continuation of LFM therefore, requires the presence of an asymmetry between the two grains on each side of the liquid film. Such an asymmetry can arise from the difference in stress produced by lattice diffusion of a solute from the interface due to anisotropy of the elastic constants. A diffusive flux across the liquid layer will be established and this will carry material from the more highly stressed solid to the less highly stressed solid at the expense of the more highly stressed solid. The surface energy due to the curvature of the grain faces can also alter the local equilibrium at each of the grain faces providing a diffusive flux in the liquid. Yoon et al. [130,131] using the results of Larche and Cahn [132] on the effect of self-stress in a crystalline solid on the composition of another phase in equilibrium, provided a detailed analysis of the kinetics of liquid film migration incorporating the effects of interface curvature. Their derivation is presented in detail in the subsequent paragraphs.

The equilibrium solubility in a fluid of a single component stressed solid with a planar surface was given by Gibbs [133]. The condition for equilibrium between binary liquid and stressed solid solutions have been derived by Larche and Cahn [132] as the simultaneous solution of two equations for the concentration of each phase. These equations have been modified by Yoon et al. $[130,131]$ to include the effect of curvature assuming isotropic surface free energy, $\sigma$. On basis of the calculated composition gradient in a liquid film between two stressed solids, Yoon et al. have determined the kinetics of Liquid film migration.

For cubic crystals in a small strain approximation, the elastic energy of a strained layer is:

$$
F_{(\text {clastic })}=Y \delta^{2}
$$

where $Y$ is an orientation dependent elastic modulus and $\delta$ is the strain. For elastic 
isotropy:

$$
Y=\frac{E}{(1-\nu)}
$$

where $E$ is the Young's modulus and $\nu$ is the Poisson's ratio.

The variation of $Y$ with orientation can be significant, even for cubic crystals. Hilliard [134] has derived a general expression for $Y$ in cubic crystals and it is observed that the maxima of $Y$ is along $<111>$ when $C_{44} / 2\left(C_{11}-C_{12}\right)>1$ and along $<100>$ when $C_{44} / 2\left(C_{11}-C_{12}\right)<1$

The parameter $\delta$ is the elastic strain in the surface layer. Two limiting values are important for the derivations. In the absence of any stress relaxation, $\delta$ is the result of the difference between the stress-free lattice parameters of the limiting surface composition $\bar{C}_{S}$ and that of the underlying material at $C_{0}$. For cubic materials with lattice parameters $a(C)$

$$
\delta=\frac{\left[a\left(\bar{C}_{S}\right)-a\left(C_{0}\right)\right]}{a\left(C_{0}\right)}
$$

If stress relaxation occurs the magnitude of $\delta$ will be reduced. In particular, if the surface layer is completely unstressed either through plastic flow or because the grain is compositionally homogeneous, then $\delta=0$. When $\mathrm{da} / \mathrm{dC}$ is constant (Vegard's law),

$$
F_{(\text {elastic })}=Y \eta^{2}\left(\bar{C}_{S}-C_{0}\right)^{2}
$$

where $\eta=(\mathrm{da} / \mathrm{dC}) / \mathrm{a}\left(C_{0}\right)$. For dilute solutions, the conditions for a stressed and curved solid in contact with its equilibrium liquid are:

$$
\mu_{1}^{0, S}-\mu_{2}{ }^{0, S}+R T \ln \frac{\bar{\Upsilon}_{1}^{S} \bar{C}_{S}}{\bar{\Upsilon}_{2}^{S}\left(1-\bar{C}_{S}\right)}+2 V_{M} Y(\hat{n}) \eta^{2}\left(\bar{C}_{S}-C_{0}\right)=\mu_{1}^{0, L}-\mu_{2}^{0, L}+R T \ln \frac{\bar{\Upsilon}_{1}^{L} \bar{C}_{L}}{\bar{\Upsilon}_{2}^{L}\left(1-\bar{C}_{L}\right)}
$$

and

$$
\mu_{2}{ }^{0, S}+R T \ln \bar{\Upsilon}_{2}^{S}\left(1-\bar{C}_{S}\right)+V_{M} Y(\hat{n}) \eta^{2}\left[\left(C_{0}\right)^{2}-\left(\bar{C}_{S}\right)^{2}\right]+2 \sigma K=\mu_{2}{ }^{0, L}+R T \ln \bar{\Upsilon}_{2}^{L}\left(1-\bar{C}_{L}\right)
$$


and the conditions for an unstressed planar solid in contact with its liquid are:

$$
\mu_{1}^{0, S}-\mu_{2}{ }^{0, S}+R T \ln \frac{\Upsilon_{1}^{S} C_{S}}{\Upsilon_{2}^{S}\left(1-C_{S}\right)}=\mu_{1}^{0, L}-\mu_{2}^{0, L}+R T \ln \frac{\Upsilon_{1}^{L} C_{L}}{\Upsilon_{2}^{L}\left(1-C_{L}\right)}
$$

and

$$
\mu_{2}^{0, S}+R T \ln \Upsilon_{2}^{S}\left(1-C_{S}\right)=\mu_{2}^{0, L}+R T \ln \Upsilon_{2}^{L}\left(1-C_{L}\right)
$$

for components 1 and 2 where $T$ is the temperature, $\mu_{i}{ }^{0, S}$ and $\mu_{i}{ }^{0, L}$ are the standard chemical potentials and $\Upsilon_{i}^{S}$ and $\Upsilon_{i}^{L}$ are the activity coefficients of the ith component in the solid and the liquid, $C_{S}$ and $C_{L}$ are the mole fractions of component 1 in the solid and liquid, $V_{M}$ is the molar volume of the solid, $\mathrm{K}$ is the mean curvature, $Y(\hat{n})$ is the orientation dependent term and a bar indicates the quantities for the stressed solid.

The above equations are derived from basic laws of thermodynamics and can be schematically illustrated on a free energy composition diagram. The first equation in each set is the condition that the tangent to the free energy curve of a solid is parallel to that of its equilibrium liquid. The second equation is the condition that the tangents to the solid and liquid curves go through a common point. The two together are the common tangent construction, shown in fig 4.29.

Equations $4.8 \mathrm{a}$ and $4.8 \mathrm{~b}$ are the equations for the composition of unstressed solid and liquid coexisting in equilibrium. Equations $4.7 \mathrm{a}$ and $4.7 \mathrm{~b}$ are the same equations when part of the solid in contact with the liquid is elastically stressed. Equations 4.7a and $4.7 \mathrm{~b}$ can be solved simultaneously, but a simple solution is obtained if it can be assumed that the changes in solubility due to stress and curvature are small. Expanding terms in equation 4.7 to a second order about the unstressed equilibrium composition and making use of equation 4.8 , the local concentration of a liquid in equilibrium with a stressed and curved solid is given by:

$$
\bar{C}_{L}=C_{L}+\left[\frac{Y(\hat{n}) \eta^{2} V_{M}\left[R T / C_{S}\left(1-C_{S}\right)\right]\left[1+\partial \ln \Upsilon^{S} / \partial \ln C_{S}\right]}{2 Y(\hat{n}) \eta^{2} V_{M}+\left[R T / C_{S}\left(1-C_{S}\right)\right]\left[1+\partial \ln \Upsilon^{S} / \partial \ln C_{S}\right]}\left(C_{S}-C_{0}\right)^{2}+2 \sigma K V_{M}\right] \mathrm{X}
$$




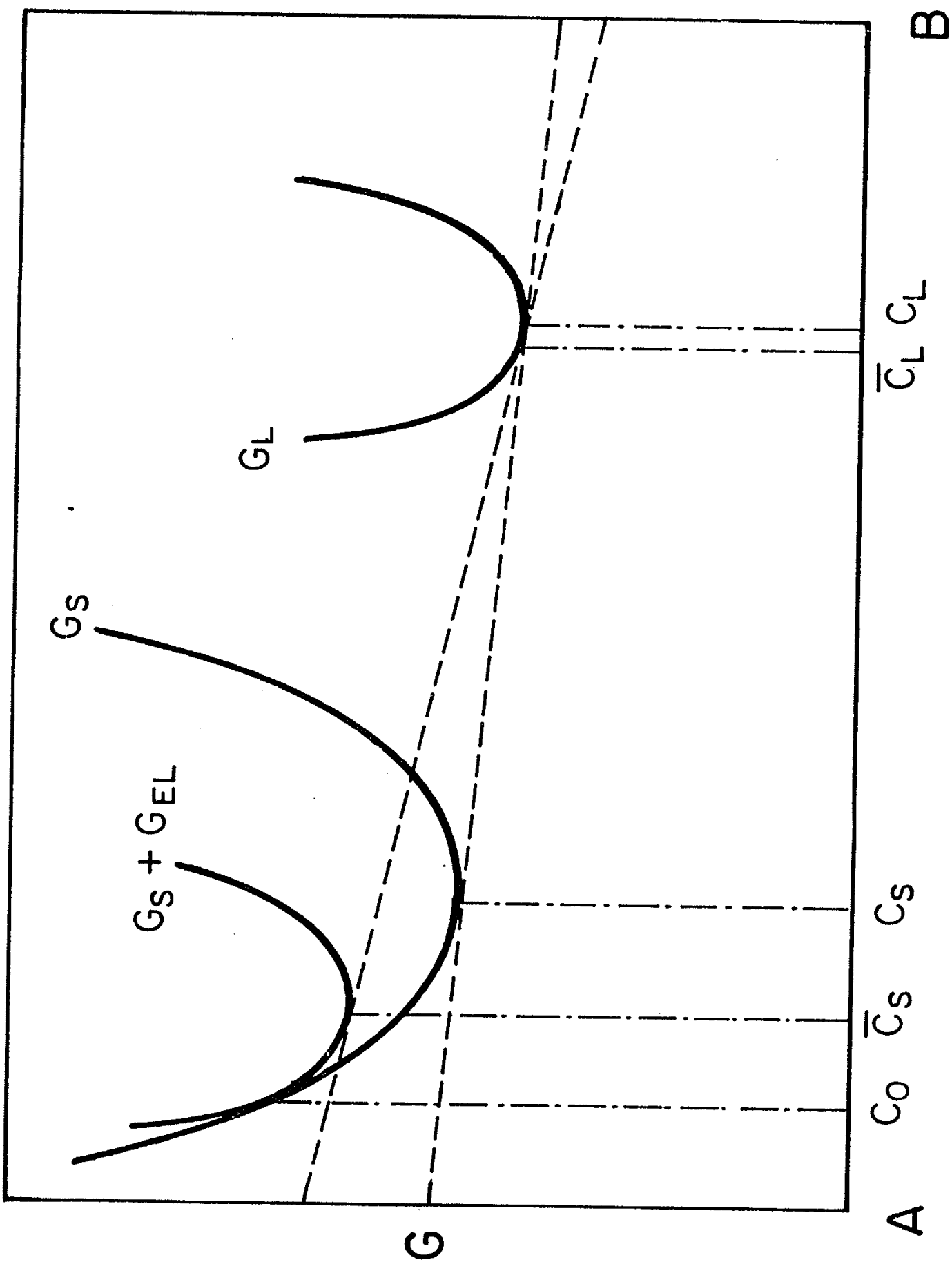

Fig. 4.29 : Common tangent construction for an unstressed planar solid and a coherently stressed solid in equilibrium with liquid $[130,131]$. 


$$
\frac{C_{L}\left(1-C_{L}\right)}{R T\left(C_{L}-C_{S}\right)}\left[1+\frac{\partial \ln \Upsilon^{S}}{\partial \ln C_{L}}\right]^{-1}
$$

in terms of the concentrations for an unstressed planar solid $C_{S}$ in equilibrium with a liquid at $C_{L}$. The equilibrium depends on the local orientation $\hat{n}$, the local curvature, and on the concentration $C_{0}$ of the underlying solid that determines the strain.

In equation 4.9 , the term which includes the elastic energy and the free energy of mixing of the solid is a driving force for boundary motion in the same sense that the curvature term is a driving force for boundary migration. Through the elastic energy term, the coupling is provided between the free energy of mixing due to diffusion at the interface and the boundary motion. The fraction of free energy of mixing of the solid that is available for boundary migration, $\phi$, is seen in equation 4.9 to be determined by the relative magnitudes of the elastic energy and the free energy of mixing. The maximum value that the driving force may achieve for a binary solution is

$$
\left[\frac{R T}{2 C_{S}\left(1-C_{S}\right)}\right]\left[1+\frac{\partial \ln \Upsilon^{S}}{\partial \ln C_{S}}\right]\left(C_{0}-C_{S}\right)^{2}
$$

For a binary solution the general expression for $\phi$ is:

$$
\phi=\frac{2 Y(\hat{n}) V_{M} \delta^{2}}{2 Y(\hat{n}) V_{M} \delta^{2}+\left[R T / C_{S}\left(1-C_{S}\right)\right]\left[1+\partial \ln \Upsilon^{S} / \partial \ln C_{S}\right]\left(C_{S}-C_{0}\right)^{2}}
$$

For Vegard's law $(\eta=$ constant $), \phi$ becomes independent of $\left(C_{S}-C_{0}\right)$,

$$
\phi=\frac{2 Y(\hat{n}) V_{M} \eta^{2}}{2 Y(\hat{n}) \eta^{2} V_{M}+\left[R T / C_{S}\left(1-C_{S}\right)\right]\left[1+\partial \ln \Upsilon^{S} / \partial \ln C_{S}\right]}
$$

When

$$
Y(\hat{n}) \eta^{2} V_{M}<<\left[\frac{R T}{2 C_{S}\left(1-C_{S}\right)}\right]\left[1+\frac{\partial \ln \Upsilon^{S}}{\partial \ln C_{S}}\right]
$$




$$
\phi=\frac{2 Y(\hat{n}) V_{M} \eta^{2}}{\left[R T / C_{S}\left(1-C_{S}\right)\right]\left[1+\partial \ln \Upsilon^{S} / \partial \ln C_{S}\right]}
$$

and equation 4.9 becomes:

$$
\bar{C}_{L}=C_{L}+\left[Y(\hat{n}) \eta^{2} V_{M}\left(C_{S}-C_{0}\right)^{2}+2 \sigma K V_{M}\right]\left[\frac{C_{L}\left(1-C_{L}\right)}{R T\left(C_{L}-C_{S}\right)}\right]\left[1+\frac{\partial \ln \Upsilon^{L}}{\partial \ln C_{L}}\right]^{-1}
$$

and when

$$
Y(\hat{n}) \eta^{2} V_{M}>>\left[\frac{R T}{2 C_{S}\left(1-C_{S}\right)}\right]\left[1+\frac{\partial \ln \Upsilon^{S}}{\partial \ln C_{S}}\right]
$$

equation 4.9 becomes:

$$
\bar{C}_{L}=C_{L}+\left[\frac{R T}{2 C_{S}\left(1-C_{S}\right)}\left(1+\frac{\partial \ln \Upsilon^{S}}{\partial \ln C_{S}}\right)\left(C_{S}-C_{0}\right)^{2}+2 \sigma K V_{M}\right] \frac{C_{L}\left(1-C_{L}\right)}{R T\left(C_{L}-C_{S}\right)}\left[1+\frac{\partial \ln \Upsilon^{L}}{\partial \ln C_{L}}\right]^{-1}
$$

The free energy diagram which correspond to these limits are shown schematically in figs $4.30(a, b)$. With the assumption of Henrian behavior $(\partial \Upsilon / \partial C=0)$ equations 4.13 and 4.14 become:

$$
\bar{C}_{L}=C_{L}+\left[Y(\hat{n}) \eta^{2}\left(C_{S}-C_{0}\right)^{2}+2 \sigma K\right] \frac{V_{M}}{R T} \frac{C_{L}\left(1-C_{L}\right)}{\left(C_{L}-C_{S}\right)}
$$

and

$$
\bar{C}_{L}=C_{L}+\left[\frac{R T}{2} \frac{\left(C_{S}-C_{0}\right)^{2}}{C_{S}\left(1-C_{S}\right)}+2 \sigma K V_{M}\right] \frac{C_{L}\left(1-C_{L}\right)}{R T\left(C_{L}-C_{S}\right)}
$$

Equations 4.7 and 4.8 are general thermodynamic relationships describing the effects of curvature and coherency strain on equilibrium compositions in binary liquid and solid systems, under the assumptions of isotropic surfaces and small strains. The kinetics of 

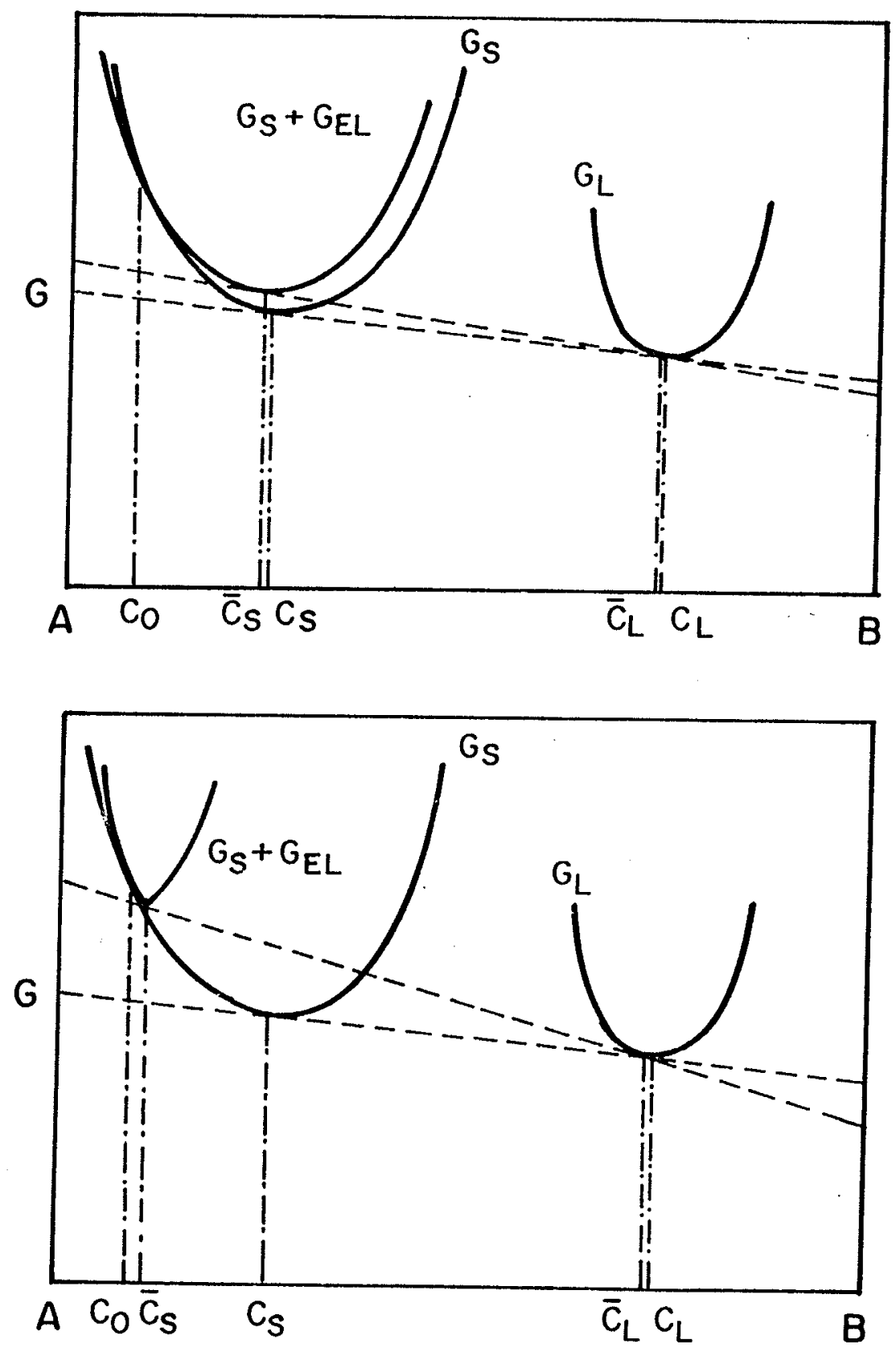

Fig. 4.30 : Common tongent construction for a coherent solid with ( $a$ ) a low and ( $b$ ) a high elastic energy in equilibrium with liquid $[130,131]$. 
liquid film migration can now be treated as a boundary value problem for diffusion through the liquid using the liquid concentration, equation 4.9 as derived from equations 4.7 and 4.8 to provide boundary conditions for the flux equation. Assuming negligible convection in the liquid, diffusion described by Fick's law and conservation of mass at the interface give a film velocity:

$$
v=\frac{D_{L}}{\left(C_{L}-C_{S}\right)} \operatorname{grad} C_{L}
$$

where $D_{L}=$ diffusivity in the liquid. When the film thickness is small, equation 4.17 can be approximated as:

$$
v=\frac{D_{L}}{\left(C_{L}-C_{S}\right)} \frac{\left(\bar{C}_{L}^{A}-\bar{C}_{L}^{B}\right)}{\lambda}
$$

where $\bar{C}_{L}^{A}-\bar{C}_{L}^{B}$ is the concentration difference in the liquid between the two interfaces and $\lambda$ is the film thickness.

In the preceding, the equilibrium compositions of the liquids as functions of curvature and stress in their equilibrium solids, and the velocity as a function of difference in liquid compositions between two liquid-solid interfaces, were derived from the basic laws of thermodynamics and kinetics. These expressions can now be applied on a case by case basis depending on the characteristics of the system under examination. For simplicity, in the following analysis of liquid film migration, the small strain and Henrian solution behavior are assumed to apply. Equation 4.15 can then be used to calculate the difference in the equilibrium liquid concentrations across a film in contact with two solids that are curved and stressed and have different values of $Y$. If the two solids are of the same phase but are of different initial compositions, $C_{0, A}$ and $C_{0, B}$ and have different elastic coefficients, $Y\left(\hat{n}_{A}\right)$ and $Y\left(\hat{n}_{B}\right)$, and different curvatures $K_{A}$ and $K_{B}$, the concentration difference between liquids becomes:

$$
\bar{C}_{L, A}-\bar{C}_{L, B}=\frac{V_{M}}{R T}\left[Y\left(\hat{n}_{A}\right) \eta^{2}\left(C_{S}-C_{0, A}\right)^{2}-Y\left(\hat{n}_{B}\right) \eta^{2}\left(C_{S}-C_{0, B}\right)^{2}+2 \sigma\left(K_{A}-K_{B}\right)\right] \frac{C_{L}\left(1-C_{L}\right)}{\left(C_{L}-C_{S}\right)}
$$


An additional assumption can be made to simplify these expressions for the case of LFM. In LFM, the initial composition of the solid is uniform $\left(C_{0, A}=C_{0, B}=C_{0}\right)$. This assumption reduces equation 4.19 to:

$$
\bar{C}_{L, A}-\bar{C}_{L, B}=\frac{V_{M}}{R T}\left[\left(Y\left(\hat{n}_{A}\right)-Y\left(\hat{n}_{B}\right)\right) \eta^{2}\left(C_{S}-C_{0}\right)^{2}+2 \sigma\left(K_{A}-K_{B}\right)\right] \frac{C_{L}\left(1-C_{L}\right)}{\left(C_{L}-C_{S}\right)}
$$

When the stress is completely relaxed in grain $B$, the elastic energy goes to zero in that grain and

$$
\bar{C}_{A}^{L}-\bar{C}_{B}^{L}=\frac{V_{m}}{R T}\left[\left(Y\left(\hat{n}_{A}\right) \eta^{2}\left(C_{S}-C_{0}\right)^{2}+2 \sigma\left(K_{A}-K_{B}\right)\right] \frac{C_{L}\left(1-C_{L}\right)}{\left(C_{L}-C_{S}\right)}\right.
$$

For two stressed and curved solids separated by a thin liquid film, equation 4.20 applies and the velocity of the film is:

$$
v=\frac{D_{L} V_{n}}{\lambda R T}\left[\left(Y\left(\hat{n}_{A}\right)-Y\left(\hat{n}_{B}\right)\right) \eta^{2}\left(C_{S}-C_{0}\right)^{2}+2 \sigma\left(K_{A}-K_{B}\right)\right] \frac{C_{L}\left(1-C_{L}\right)}{\left(C_{L}-C_{S}\right)^{2}}
$$

For a stressed and curved solid separated by a thin liquid layer from an unstressed and curved solid, equation 4.21 applies and the velocity of the liquid film is:

$$
v=\frac{D_{L} V_{m}}{\lambda R T}\left[\left(Y\left(\hat{n}_{A}\right) \eta^{2}\left(C_{S}-C_{0}\right)^{2}+2 \sigma\left(K_{A}-K_{B}\right)\right] \frac{C_{L}\left(1-C_{L}\right)}{\left(C_{L}-C_{S}\right)^{2}}\right.
$$

If Vegard's law did not hold, equations 4.15, 4.19-4.23 could be rederived to yield equations that are identical except that $\delta$ appears in the equations instead of $\eta\left(C_{S}-C_{0}\right)$. The thermodynamic properties of the solid solution have disappeared from all these equations as a result of the small $\phi$ approximation. This simplification of the driving force term continues to hold in a derivation of these equations for multicomponent systems in the small $\phi$ approximation. In LFM, equations 4.20 and 4.22 describe the liquid concentrations and the velocity during initiation of migration before any stress relaxation occurs in either grain. Equations 4.21 and 4.23 describe the concentration of solute in the liquids and velocity of the film migration after stress relief has occurred in one grain but not in the adjacent grain. 
The following paragraphs will discuss the limiting cases of the above derived theory. In considering the initiation of film motion, it is assumed that both the grains are initially homogeneous and stress free. When solid state diffusion begins, differences in stresses force the film to migrate into the more highly stressed solid. Two reasons for stress differences are discussed, those due to differences in $Y(\hat{n})$ and those due to stress relaxation.

After considerable film migration has occured, it is assumed that the growing grain is stress free while the dissolving grain remains coherently stressed. Then, the anisotropy of velocity is independent of the growing grain, but depends on the orientation of the dissolving grain through $Y(\hat{n})$.

If, for initially equilibrated samples, the conditions are changed so that the solid solution is no longer in equilibrium with the liquid, there will be a transient after which the liquid composition in the liquid film reaches a steady state profile whose boundary values are in equilibrium with the stressed surface layers of the solids. Reaching the steady state condition requires a time of the order of $\lambda^{2} / D_{L}$. Thereafter, the liquid film should migrate with a velocity given by equation 4.22 . If the curvature is made negligible by prior stabilization of the microstructure, the direction of migration is into the grains with larger $Y(\hat{n})$ for cubic crystals.

The stresses due to coherency can relax by plastic flow and this, as indicated in equations 4.22 and 4.23 , will affect the velocity. While local dislocation motion can affect the stresses quite early, dislocation multiplication adequate to reduce the stress markedly cannot occur until the diffusion layer has reached a critical thickness $L_{C}[135,136]$. This is because the elastic energy in the diffusion layer is proportional to its thickness, while the energy of the dislocations needed to reduce the stress is, to a first approximation, independent of thickness. Because $L_{C}$ is inversely proportional to $Y$, stress relaxation would occur first in the more highly stressed grain, were it not for the effects of the film velocity on the thickness of the diffusion layer.

Under non-ideal conditions, the stress relaxation will not occur uniformly along the 
surface of the grains, but depends on the preexisting dislocations, dislocation sources and dislocation nucleation. During the process of stress relaxation, these variations in stress will produce corresponding variations in velocity. In extreme cases of closely matched values of $Y$, little motion occurs until stress relaxation occurs locally. The liquid film migration would occur in the vicinity of each such site of stress relaxation leading to local bowing out of the interface.

A characteristic diffusion layer thickness, $L$, in the absence of film migration is of the order of $\sqrt{D_{S} t}$, where $D_{S}$ is the lattice diffusivity of the solute and $\mathrm{t}$ is the time. At long times, for a constant liquid film migration velocity, $L$ will be characterized by a steady state thickness $\left(D_{S} / v\right)$ in the dissolving grain. The diffusion layer thickness in the growing grain will be:

$$
L=\sqrt{D_{S} t}+v t
$$

which at long times reduces to $L=v t$. In the dissolving grain, $L$ will be of the form:

$$
L=\sqrt{D_{S} t}\left[\frac{\sqrt{D_{S} t}}{\sqrt{D_{S} t}+v t}\right]
$$

For $\sqrt{D_{S} t}>>v t, \quad L=\sqrt{D_{S} t}$ and for $\sqrt{D_{S} t}<<v t, L=D_{S} / v$. The initiation stage through the time for coherency loss can now be summarized. After the initial transient the velocity will be given by equation 4.22 until coherency loss occurs. Some dislocation motion will reduce the stress and thus the velocity, possibly leading to local bowing out of the interface. If the velocity given by equation 4.22 is greater than $D_{S} / L_{C}$, coherency loss will occur after sometime in the growing grain. Thereafter the velocity of liquid film migration will increase to that given by equation 4.23 .

However since $L_{C}$ is proportional to $Y$, and $v$ depends on many factors, such as solubility in the fluid and the elastic anisotropy described by the term $\left(Y\left(\hat{n}_{A}\right)-Y\left(\hat{n}_{B}\right)\right)$, it is quite possible that $v<<D_{S} / L_{C}$. In that case, the diffusion layer thicknesses are affected little by $v$ and coherency losses should occur in the grain with the higher Y. Liquid film migration would first be in the direction of higher $Y$ and then would change direction. 
If the initial curvatures are so large that $2 \sigma\left(K_{A}-K_{B}\right)$ term is dominant in equation 4.22 , the initial symmetry breaking and, hence, the direction of migration can be determined by the curvatures.

An interesting theoretical problem occurs if $D_{S} / v$ is smaller than the atomic size. Under these conditions, no diffusion into the solid occurs. Thermodynamic restrictions exist for the liquid composition in contact with the solid. There is a thermodynamic limit to the liquid composition $C_{L}^{1}$ if the dissolution of the solid $C_{0}$ is to be spontaneous, as indicated in figure 4.31. There is another limiting composition given by equation 4.9 in which the liquid $\bar{C}_{L}$ is in equilibrium with a coherent layer on this solid. Beyond this limit the solid can grow spontaneously. But these two limiting liquid compositions are different and in the presence of liquid of intermediate composition, thermodynamics permits both growth and dissolution of solid. In contrast, the equilibria of equation 4.8 are reversible. A slight deviation in liquid composition in one direction from $C_{L}$ in figure 4.31 permits only dissolution and in the other direction only growth. Only at exact equilibrium are both growth and dissolution permitted. Yoon et al $[130,131]$ believe the liquid composition that will be obtained in the high velocity case is determined by the kinetic laws which are subject to these thermodynamic limits.

We will now examine the situation of steady state growth after coherency loss following further the arguments of Yoon et al. [130,131]. After coherency loss, the growing grain is stress free while the dissolving grain remains stressed if the thickness $D_{S} / v$ exceeds a few times the atomic size. Under these conditions the velocity of growth depends only on the anisotropy of $Y(\hat{n})$ of the dissolving grain. It is independent of the orientation of the growing grain, or of their orientation relationship. From this orientation dependence, the shapes of grains are predicted as a function of anisotropy of the elastic constants. The shapes which evolve are analogous to those observed during crystal growth where the directions of fastest growth become corners in the final shape and directions of the slowest growth becomes planes. 


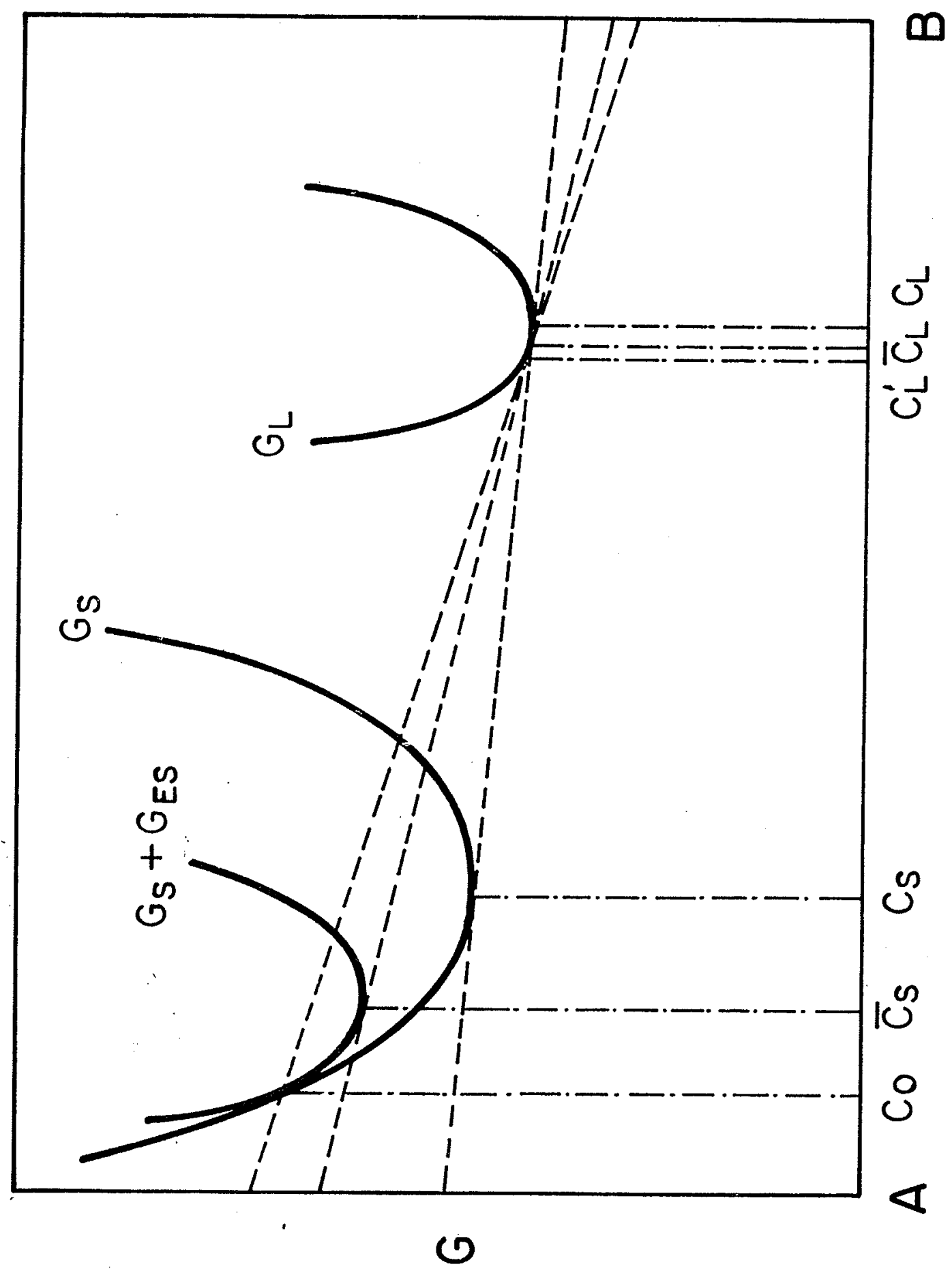

Fig. 4.31 : Graphical construction of limiting liquid compositions for spontoneous dissolution of solids with $\mathrm{C}_{\mathrm{O}}, \overline{\mathrm{C}}_{\mathrm{S}}$ and $\mathrm{C}_{\mathrm{S}}[130,131]$. 
Finally, before discussing the results of the present investigation in terms of the coherency strain model, let us examine the coherency strain in ternary alloys. From the coherency strain model, no migration should occur when the coherency strain is reduced to zero. In most of the binary alloys, the lattice parameter changes monotonically with composition. Hence, the coherency change would be zero only when there is no composition change. In ternary alloys, however, the lattice parameter can remain constant over a wide range of composition. If the lattice parameter of a ternary alloy follows the 'rule of mixtures', the strain $\delta$ due to a composition change from $\left(C_{01}, C_{02}\right)$ to $\left(C_{1}, C_{2}\right)$ will be

$$
\delta=\eta_{1}\left(C_{1}-C_{01}\right)+\eta_{2}\left(C_{2}-C_{02}\right)
$$

where $\eta_{1}$ and $\eta_{2}$ are constants. Hence $\delta$ can be positive or negative regardless of the signs of $\eta_{1}$ and $\eta_{2}$, and becomes zero at certain combinations of $\left(C_{1}-C_{01}\right)$ and $\left(C_{2}-C_{02}\right)$. On the other hand, the free energy of mixing for the composition change will be greater than zero if the composition range lies outside the spinodal. Therefore, in ternary alloys, the coherency strain may be reduced to zero even when the free energy of mixing remains finite.

From the equations governing the kinetics of LFM, we see that if the initial curvatures of the solid-liquid interfaces are significant, then the $2 \sigma\left(K_{1}-K_{2}\right)$ becomes the dominant term and dictates the direction of migration as well as increasing the migration velocity. One can see from the micrographs presented that the curvature of the fine recrystallized grains is significant and hence the velocity is enhanced by the curvature term $2 \sigma\left(K_{1}-K_{2}\right)=$ $4 \sigma \bar{K}$, where $\bar{K}$ is the mean curvature of the grain boundary under consideration. However, in the case of long and straight interfaces with no significant curvature, the velocity of migration is given by

$$
v=\frac{D_{L} V_{m}}{\delta R T}\left[E \eta^{2}\left(C_{S}-C_{0}\right)^{2}\right] \frac{C_{L}\left(1-C_{L}\right)}{\left(C_{L}-C_{S}\right)^{2}}
$$


which is significantly less as compared with the interfaces that have substantial curvatures. The concentration difference $\Delta C$ between the two interfaces is considerably increased by the curvature term and thus, for interfaces with significant curvature, greater driving force becomes available facilitating easy initiation of LFM. The velocity of migration of LFM after coherency loss is also significantly increased in these cases. This is the prime reason for the occurrence of substantial LFM along grain boundaries of fine recrystallized grains as opposed to along long, straight warm worked boundaries or recrystallized grain boundaries with no signficant curvature.

Recently Patterson and Liu [137] have experimentally determined the relationship between mean grain boundary curvature and the mean grain size. They observed the mean grain boundary curvature to be linearly related to the inverse of mean grain intercept. The value of the ratio of average mean grain boundary curvature to the inverse of mean grain intercept was found to be 0.31 for pure aluminum throughout the grain growth. Patterson and Liu's work provides experimental evidence to the present suggestion that fine grains possess substantial mean grain boundary curvature. Hence, the occurrence of extensive LFM is observed along fine grains in the HAZ.

Brechet and Purdy [138] have formulated approximate expressions for liquid film velocity under the limiting cases of (i) no lateral transport of solute from bulk liquid matrix to intergranular liquid film i.e under the condition where the volume of the liquid between each grain pair is fixed (ii) seeking a steady state and allowing for solute transport from the bulk liquid which is taken to act as a solute reservoir in equilibrium with an average external curvature. They had suggested based on the observation of parabolic dependence of migration velocity on the elastic misfit observed by Yoon et al. $[119,122,125]$ and the fact that $v=0$ for zero coherency strain, that the experiments of Yoon et al. correspond to nonsteady state LFM. The experiments of Yoon et al. were performed under isothermal conditions where there always existed a constant solute sink. However, in the case of welding, large thermal gradients exist. For a heat input of $1.38 \mathrm{KJ} / \mathrm{cm}$, 
implanted thermocouples in the present work showed cooling rates in the fusion zone and $0.02 \mathrm{~cm}$ from the fusion boundary to be $1315^{\circ} \mathrm{C} / \mathrm{sec}$ and $1038^{\circ} \mathrm{C} / \mathrm{sec}$ respectively over the temperature range of $1300^{\circ} \mathrm{C}$ to $950^{\circ} \mathrm{C}$. Hence, in the partially melted zone adjacent to the fusion boundary the grain boundaries on cooling are only above the austenite-niobium carbide eutectic (approximately $1290^{\circ} \mathrm{C}$ from D.S.C work) for about $0.1 \mathrm{sec}$.

The extent of grain boundary liquation observed and the concentration of niobium and carbon observed on the HAZ grain boundaries indicate that the grain boundary liquid is more enriched in solutes with partition coefficients less than unity as compared to initial liquid in the molten fusion zone. The initial liquid in the molten fusion zone is however, marginally enriched in niobium and carbon as compared to the bulk matrix composition due to the melting of the large MC carbide/carbonitride insolubles during the weld heating cycle. Significant lateral diffusion of solutes with partition coefficients greater than unity is not expected to occur from the molten fusion zone to the $H A Z$, as gamma dendrites start forming with the onset of cooling. In the solidifying fusion zone, with the onset of $\gamma / M C$ monovariant eutectic, there is considerable dendrite interlocking and trapping of interdendritic liquid in the $\gamma$ dendritic and $M C$ interdendritic intersticies. If attainment of steady state conditions is suspect even when an unhindered path from the liquid sink exists, it is highly unlikely that such a possibility exists under the present conditions. Hence, substantial lateral flow of interdendritic liquid may not occur along contiguous grain boundaries into the HAZ. Furthermore, as mentioned earlier, if constitutional liquation were not to be the cause for the formation of liquid on the HAZ boundaries, no grain boundary LFM would ever be observed in synthetic HAZ specimens. Microstuctural evidence abounds in the literature (e.g., see ref 1 ) that show the occurrence of grain boundary LFM in synthetic HAZ specimens.

In actual welding, some contribution from backfilling may exist in areas very close to the fusion boundary if the liquid in the fusion zone has good wettability so that complete wetting of the boundary can occur. Approximate calculations can be made as to the 
upper limit of the extent of backfilling based on expressions from classic surface tension theory [139]. The extent of backfilling is governed by the rate of penetration of capillary spaces. For early stage of penetration into a capillary space of any inclination, the time $t$, to reach a height $h$ can be written as

$$
t=\frac{2 \eta h^{2}}{r \Upsilon \cos \theta}
$$

or alternatetely,

$$
h^{2}=\frac{\operatorname{tr} \Upsilon \cos \theta}{2 \eta}
$$

where $\Upsilon$ is the surface tension of the liquid, $\theta$ is the contact angle, $r$ is the radius of the capillary and $\eta$ is the viscosity of the liquid. To a first approximation, assuming the grain boundary as a capillary $(r=10 \AA)$ and taking the values of $\Upsilon$ as 1500 ergs $/ \mathrm{sqcm}$ (surface tension of $\mathrm{Fe}-50 \% \mathrm{Ni}$ alloy at melting point) [140], $\theta$ as zero (the worst case), $\mathrm{t}$ $=0.1 \mathrm{sec}$ (maximum time during which some liquid exists in the fusion zone $[80,81]$ ) and $\eta$ as 2.25 centipoise (the viscosity of $\mathrm{Fe}-2.5 \% \mathrm{C}$ alloy at $1400^{\circ} \mathrm{C}$ ) [140], the upper limit of backfilling is calculated to be $183 \mu \mathrm{m}$. However, as can be observed from the micrographs (figs. 4.24 and $4.27(\mathrm{a})$, grain boundary liquation and LFM occurs to the extent of 300 to $400 \mu \mathrm{m}$ in the HAZ. Hence, the extent of LFM observed on these boundaries provides further conclusive evidence that the liquid on the boundaries primarily originated from the constitutional liquation of preexisting precipitates.

A few comments are in order as to the calculation of the upper limit of backfilling. The calculated distance $h$ has to be less than or equal to the equilibrium height $H$ which is determined by the contact angle, inclination and radius of the capillary, surface tension and density of the liquid and acceleration due to gravity. Under the absence of any other forces than gravity, equilibrium can never be reached in a horizontal capillary of finite length. However, in the case of fusion welding, the grain boundaries in the HAZ are under the influence of thermal and mechanical restraints and these forces, depending on the inclination of the grain boundary can either increase or decrease the equilibrium penetration 
distance, $H$. Minimal backfilling was observed on the boundaries lying perpendicular to the fusion line; however some backfilling could be observed (fig 4.32) on warm worked boundaries lying at an inclination to the fusion line.

Even if some backfilling were to exist, as mentioned above, the majority of the liquid that is penetrated is of composition marginally greater in niobium and carbon as compared with the bulk level and this liquid can also relieve its supersaturation, by LFM, if conditions favor for its occurrence; else it resolidifies as niobium enriched gamma accompanied by precipitation of some fine MC carbides. If the latter case were to occur, as can be seen from the figure, there is a gradual decrease in the width of the resolidified region as one proceeds from fusion line further down into the HAZ. The occurrence of LFM can be distinguished from the increased width of the migrated slabs close to the fusion line and also in areas remote from fusion line (fig 4.27). In areas remote from the fusion line, liquid penetration along grain boundaries in areas remote from the fusion line, cannot be due to capillarity effects (backfilling from fusion zone). The liquid in these instances could have only originated from the constitutional liquation of prior precipitates rich in niobium on the grain boundaries. Hence these observations prove in a confirmatory manner the occurrence of constitutional liquation on HAZ grain boundaries in Incoloy 903.

The size of MNP-type phosphides on the long warm worked grain boundaries is expected to be larger than those on the recrystallized boundaries which form only in the intermediate stages of thermomechanical processing. Formation of most of the fine recrystallized grains occurs much later in the process of thermomechanical treatment. Thus, the total time available for precipitate growth is much shorter on fine recrystallized grains as opposed to the warm worked grains. Hence, the precipitate size on warm worked grains is expected to be larger. When constitutional liquation occurs, the concentration of phosphorus on the liquated warm worked boundaries is thus expected to be higher than on the recrystallized boundaries. It is also well docummented in the literature that both sulfur and phosphorus tend to adsorb to grain boundaries and interfaces in nickel and austenitic 



fig 4.32(a): Optical micrograph illustrating liquation and microfissuring in HAZ slightly remote from the fusion line. Note some amount of backfilling occurring in regions slightly close to the fusion line. fig 4.32(b): SEM micrograph illustrating liquation and microfissuring in HAZ slightly remote from the fusion line. Some amount of backfilling occurring in regions slightly close to the fusion line is also evident. 
stainless steels. The preferential adsorption of the excess phosphorus to solid/liquid interfaces on the warm worked boundaries leads to a total decrease in the coherency strains as well a decrease in the solid-liquid cohesion. The decreased coherency strains makes the initiation of LFM on these boundaries difficult. The coherency strains are substantially decreased due to the adsorption of phosphorus because phosphorus has an atomic size much smaller than that of the the gamma matrix while niobium has an atomic size much larger than that of the gamma matrix.

There are two other aspects of LFM that need mention. In absence of significant interfacial curvature, the coupling between the available free energy of mixing and initiation of LFM is thought to be completely provided by the coherency strains arising due to the asymmetry of the elastic constants between the two solid grains seperated by the liquid film. Warm working leads to a highly textured microstructure with a preferred orientation and hence there might not exist any difference in the elastic constants between two adjoining warm worked grains. This makes initiation of LFM on warm worked boundaries further difficult or even impossible.

During LFM, it is assumed that there is complete relaxation of coherency stresses and the growing grain is stress free. If complete stress relaxation were not to occur, the velocity of LFM is reduced. It is not understood at present how the thermal stresses affect the process. However, thermal and mechanical constraints are known to be more effective on large grain boundaries lying perpendicular to the fusion line as opposed to fine grains and boundaries lying at an inclination to the fusion line. This factor may also have an effect in reducing the occurrence of LFM on large warm worked grain boundaries lying perpendicular to the fusion line. 


\section{5: GRAIN BOUNDARY EMBRITTLEMENT}

In the preceding section, the reasons for the occurrence of extensive LFM along fine recrystallized grains in the HAZ of Incoloy 903 was discussed in the light of the theoretical analysis of LFM. As discussed in section 4.3, extensive $H A Z$ microfissuring and minimal LFM occurred along the warm worked grains. The reasons for the nonoccurrence of LFM along warm worked grain boundaries was explored in the preceding section. As discussed earlier in the literature review, segregation of certain elements to the grain boundaries may substantially increase the HAZ microfissuring susceptibility by:

(i) forming low melting liquid films and thus increasing the temperature range of solidification;

(ii) increasing the wettability of the liquid by decreasing the solid-liquid interfacial energy facilitating their easy spreading along the grain boundaries;

(iii) promoting the formation of an embrittling liquid along the grain boundaries causing grain boundary liquid metal embrittlement;

(iv)embrittling the grain boundaries in solid state by decreasing the grain boundary energy and thus the grain boundary cohesive strength.

Grain boundary segregation is the phenomenon that leads to the occurrence of all the above mentioned factors. Hence, it is necessary to discuss the phenomenon of grain boundary segregation and embrittlement in order to understand the mechanism(s) of HAZ microfissuring. This section discusses in brief the literature on grain boundary segregation and embrittlement followed by a detailed examination of the nature and affect of segregation of the principle trace elements phosphorus, sulfur and boron. The section is concluded with a brief review of the phenomenon of liquid metal embrittlement.

Alloying elements and undesirable impurities often segregate to external surfaces (solid-vapor and solid-liquid interfaces) and solid-solid interfaces. Many material properties such as oxidation, corrosion, catalysis, adsorption-desorption, adhesion microhardness, wear, wetting, physical appearance, surface electrical conductivity, fatigue cracking, 
thermionic and secondary emissions, diffusion and thin film growth are strongly influenced by changes in interface and/or surface composition. Several interface properties such as grain growth, electrical as well as mechanical properties and chemical properties such as intergranular decohesion, boundary hardness, intergranular corrosion, liquid metal penetration and stress corrosion cracking have a strong dependence on the interface chemistry. Segregation to surfaces and interfaces occur due to a variety of reasons. Segregation may simply be Gibbsian type adsorption, where the reduction in surface or interface free energy is the driving force or may be of a nonequilibrium nature.

The subject of segregation has generally been treated in the restricted context of grain boundary phenomenon. However, as discussed by Hondros and Seah [141], both grain boundaries and other interfaces may be described by the concepts and laws of surface adsorption. Chemical and similar adsorption phenomena occur on all the above interfaces, the scale of the effect, however depends on parameters associated with the particular interface.

For most metallic systems, the most important interest lies in the effects of surface active impurities or residual elements which segregate to interfaces at equilibrium from very low concentrations in the bulk or vapor phases. Noting that in the dilute approximation, Henry's law holds, the generalized Gibb's adsorption equation takes the explicit form

$$
\left[\frac{d \gamma}{d X_{2}^{C}}\right]_{T}=-\frac{R T}{X_{2}^{C}} \Gamma_{2}
$$

where $X_{2}^{C}$ is the molar content of the solute and $\Gamma_{2}$ is the grain boundary excess of the dilute species, and $\gamma$ is the interfacial free energy. This is the basic equation which is used to relate composition to changes in interfacial energy for an isotropic interface. There is reasonable quantitative confirmation of the applicability of the Gibbs theorem to grain boundaries [142]. Where as earlier, $\Gamma_{2}$ for the interface was derived from $\left[d \gamma / d X_{2}^{C}\right]$, a direct knowledge of grain boundary excess of solute species allows for the determination of the fall in grain boundary energy. 
The general pattern of behavior of grain boundary energy dependence is illustrated in fig 4.33 with reference to dilute alloys [143]. In all cases, the grain boundary energy falls with increasing solute content, paralleling the behaviors observed for free surfaces and indeed for all interfaces where surface activity is important. Fig 4.33 also shows that solute species possess widely varying tendencies to adsorb to grain boundaries and reduce grain boundary energy. This tendency is referred to as grain boundary activity. Grain boundary activity is obtained from the initial slope of the grain boundary isotherm of the type shown in fig 4.33 . Thus

$$
\alpha_{b}=\left[\frac{d \gamma_{b}}{d \ln X_{2}^{C}}\right]_{T}=-\frac{R T}{X_{2}^{C}} \Gamma_{b}
$$

These measurements have been brought together into a single compilation of data via a parameter, defined as the enrichment factor, $\beta_{b}$, which is even more generally useful in describing grain boundary activity [144]. This is defined as the ratio between the grain boundary concentration $X_{b}$ and the bulk concentration $X_{2}^{C}$ of a particular species. $X_{b}$ is expressed for convenience as mole fraction of a monoatomic layer and $X_{2}^{C}$ as bulk mole fraction. Writing the standard Gibb's excess as a concentration term,

$$
\beta_{b}=\frac{X_{b}}{X_{2}^{C}}=\frac{\Gamma_{b}}{\Gamma_{b}^{0}} \frac{1}{X_{2}^{C}}=-\frac{1}{R T \Gamma_{b}^{0}}\left[\frac{d \gamma_{b}}{d \ln X_{2}^{C}}\right]_{T}
$$

where $\Gamma_{b}^{0}$ is the quantity of solute in moles per unit area constituting one monolayer.

Most of the data obtained either directly by surface analysis or by interfacial activity measurements were brought together in the plot shown in fig 4.34, between enrichment factor and the solid solubility at the temperature of measurement [144]. This direct correlation between enrichment factor and the inverse of atomic solid solubility, although noted first emperically, has more recently been shown to have a theoretical basis. Examination of the figure reveals that impurities concentrate at grain boundaries by a factor of $10^{4}$ or $10^{5}$ above bulk concentration when atomic solid solubility is low. The results of fig 4.34 also suggest that the same Hume-Rothery rules (atom size, electrovalence, crystal structure 


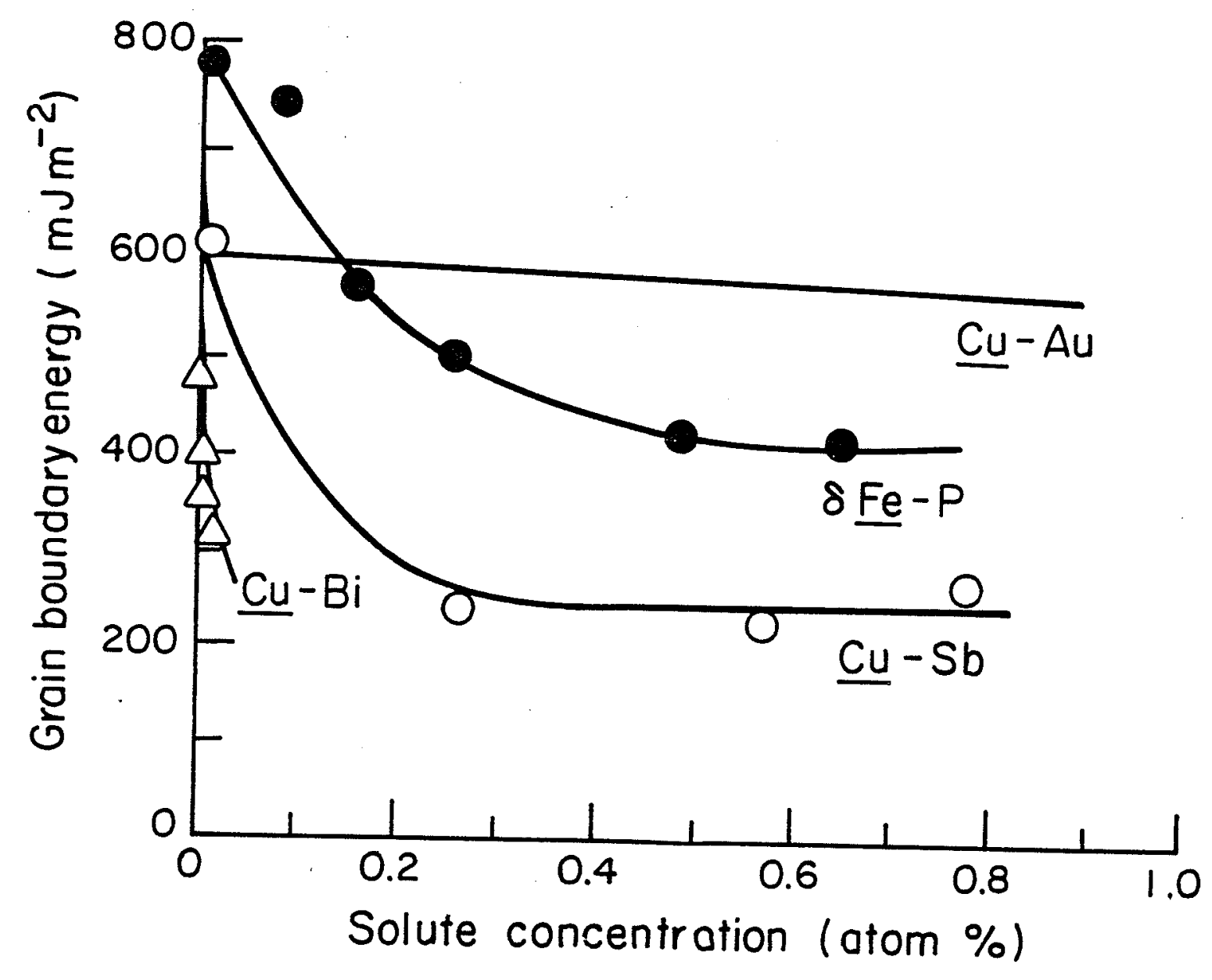

Fig. 4.33 : Variation in grain boundary energy of o ronge of binary alloys with bulk solute content [143]. 


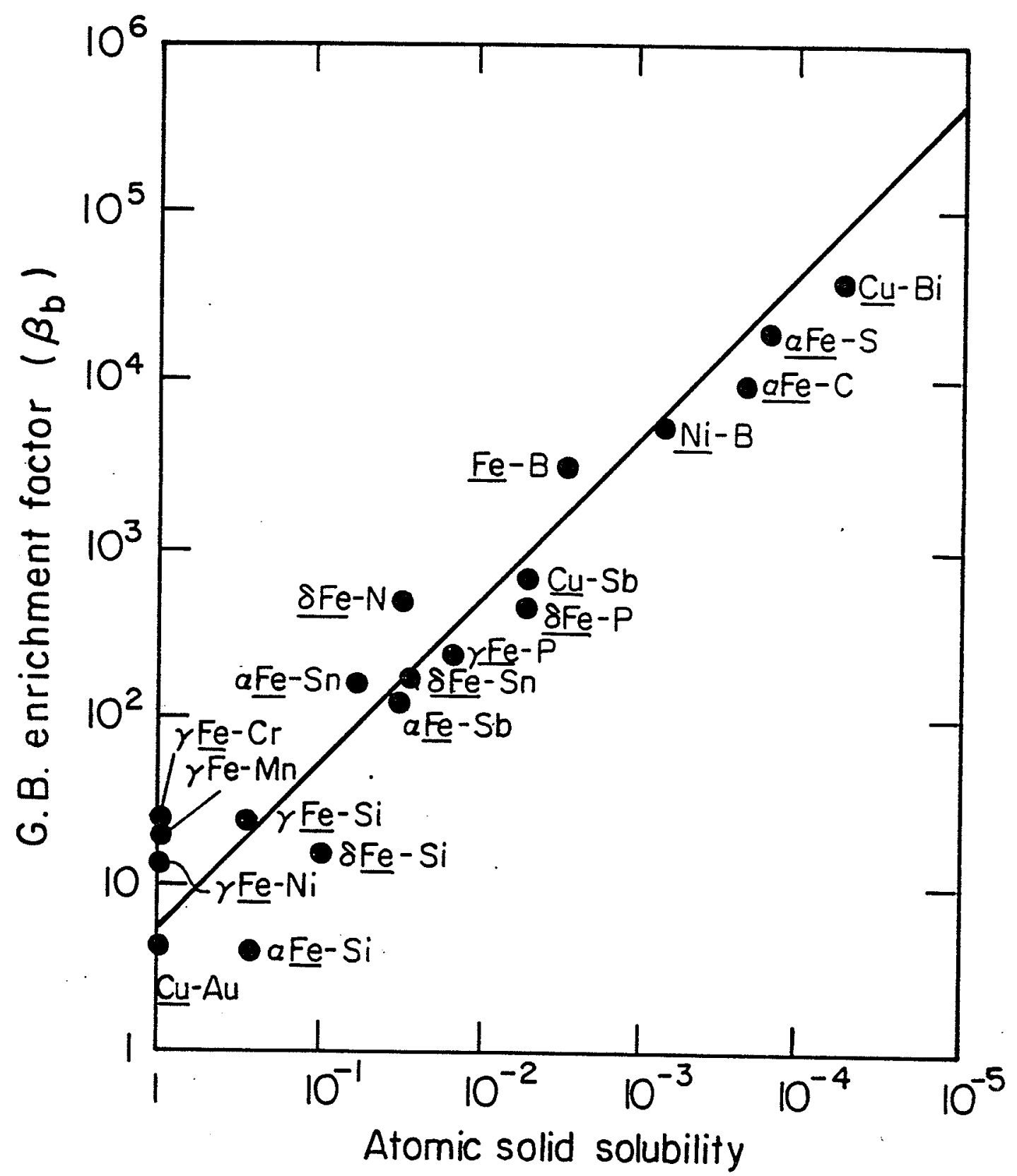

Fig. 4.34: Grain boundary enrichment factor as a function of the atomic solid solubility for binary systems. The host element is underlined in each case [144]. 
and valence electrons in the alloys) applicable to solid solubility also govern segregation behavior [145].

The correlation of fig 4.34 allows one to identify from a knowledge of equilibrium phase relationships, the principal surface active solute in a system, to determine the amount enriched at the boundary and to assess the changes in grain boundary energy resulting from this adsorption. Although the plot is based on data obtained from binary systems, as a first approximation, it can be used for multicomponent systems. Thus, knowing the precise bulk composition of the system, the procedure is to select those solutes which according to thermochemical data have a low solid solubility. From these, the corresponding values of $\beta_{b}$ are read off from the plot. In turn from equation 4.34 for $\beta_{b}$, the corresponding changes in $\gamma_{b}$ is computed for the known bulk level of solute $X_{C}$, and for the temperature of interest [142].

Hence, it is seen that strong segregation of a solute in the grain boundaries of a given solid solvent is generally expected when the former is highly surface active and or exhibits a solid solubility in the latter, which is generally the case when both species are of widely different chemical characters - e.g., a non-metallic impurity in a metallic matrix. In multicomponent systems, preferential chemical attractive interactions between solutes can result in the enhancement of the respective segregation, and can induce segregation of nonsurface active elements. This phenomenon is called cosegregation. The thermodynamics of this synergistic effect has been developed by Guttmann [146].

Guttmann's model [146] is based on regular solid solution theory and assumes that the driving force for cosegregation is an attractive chemical interaction between the cosegregating solutes. This attraction between the solutes must be greater than that between either solute and solvent. In this model the grain boundary concentration is given by

$$
X_{b i}=\frac{X_{c i} \exp \left(\Delta G_{i} / R T\right)}{1-\Sigma_{i=1}^{n=1}\left[X_{c i} \exp \left(\Delta G_{i} / R T\right)-1\right]}
$$

where $X_{b i}$ is the molar fractional monolayer segregated to the grain boundary, $X_{c i}$ is the 
bulk concentration, and $\Delta G_{i}$ for a ternary system is given by

$$
\Delta G_{1}=\Delta G_{1}^{0}+\alpha_{12}^{\prime} X_{b 2} \Delta G_{2}=\Delta G_{2}^{0}+\alpha_{12}^{\prime} X_{b 1}
$$

and

$$
\alpha_{12}^{\prime}=\alpha_{12}-\alpha_{10}-\alpha_{20}
$$

The term $\alpha$ is borrowed from the regular solution theory and suppossedly represents the difference in the attractive interactions between the two solutes and their interaction with the solvent.

The theory of multicomponent segregation shows that the effect of one component on the grain boundary segregation of another depends partly upon the effective energy of attraction or repulsion between them and partly also on whether they do, or do not, compete for the same grain boundary sites.

Impurity segregation embrittles grain boundaries if the interfacial surface energy is decreased. This reduces both the cohesive energy and the plastic work that accompanies fracture. It was demonstrated earlier that the driving force for solute segregation to interfaces is the reduction in interfacial and surface free energies. As a consequence, the reduction in grain boundary free energy occurring due to segregation or impurity adsorption will correlate with variations in fracture stress. The segregated atoms can lower the fracture strength of a grain boundary by weakening the grain boundary bonding. The following paragraphs examine the role of chemical bonding in grain boundary decohesion following the review of Briant and Banerji [147].

Clear evidence exists to the fact that segregation of certain impurity atoms to the grain boundaries leads to grain boundary decohesion [147]. In recent years some progress has been made in answering the question of why these elements cause decohesion by the application of fully quantum mechanical theoretical techniques to study the changes in chemical bonding at the grain boundaries caused by impurity atoms.

Briant and Messmer [148] have used the self-consistent field X- $\alpha$ scattered wave calculations for this work. In this procedure, a cluster of atoms is used and the molecular 
orbitals for the cluster are calculated. One can then examine the energy levels of the cluster and the molecular orbitals that correspond to those energy levels. A cluster method is particularly applicable to the study of grain boundaries since it has been shown that the structure of grain boundaries can be decomposed into clusters that often resemble distorted versions of Bernal deltahedra. One can then use these clusters for the calculations.

Messmer and Briant [149] have now reported results for calculations of iron and nickel embrittlement. They find that in all cases where a strong embrittler is added to the cluster, charge is drawn from the metal atoms on to the impurity. Thus, the metal-impurity bond is rather heteropolar or ionic. As a result of forming this bond and the consequent transfer of charge, much less charge is available for the metal-metal bonds and they will therefore be weaker. In the case of an element that enhances cohesion, e.g., when boron is present in nickel, charge is not drawn off the metal atoms and the metal impurity bond is much more covalent. Therefore, the metal-metal bonds are not weakened and an added increment of bonding occurs at the grain boundary.

A particularly striking example of this can be seen by the embrittlement of nickel by sulfur. The cluster used for this calculation was the eight atom nickel cluster shown in fig 4.35(a,b). The calculation was done with nickel atoms at the vertices and no impurity atoms and again with nickel atoms at the vertices but with a sulfur atom present in the center of the cluster. Fig 4.35(b) shows a contour plot for the total valence charge density for the cluster taken in the $y-z$ plane so that atoms $1,4,7$ and 8 are visible. Particular attention should be paid to contour 4 , which is darkened in the figures. For the $\mathrm{Ni}_{8}$ cluster it contributes to a bond between atoms 1 and 7,4 and 8 , and 7 and $8 . \ln$ the $\mathrm{Ni}_{8} \mathrm{~S}$ cluster it is greatly distorted. It now contributes to a bond between the sulfur atom and the nickel atoms nearest to it. However, it no longer makes any contribution to bonds between 1 and 7 and 4 and 8 , and the bond between these atoms will be weakened. Also, the contribution of this contour to the bond between 7 and 8 is smaller. Therefore, it is seen that as a result of introducing sulfur into the cluster, charge is drawn into the 

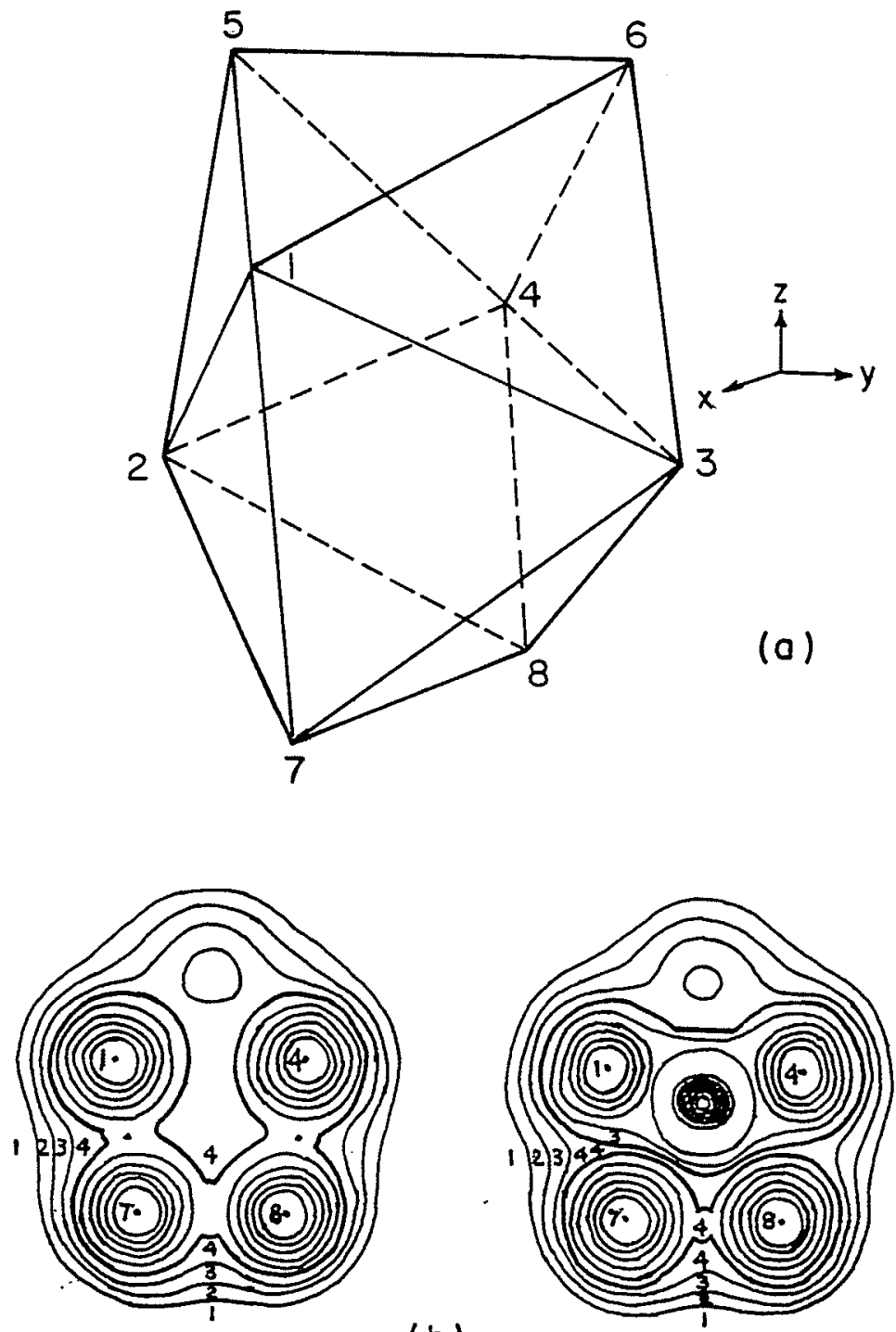

(b)

Fig. 4.35 (a): The tertahedral dodecahedron [147].

Fig 4.35 (b): The total valance change density for $\mathrm{Ni}_{8}(a)$ and NisS (b). The contours ore all equivalued.

Note in particular the change in contour number 4 for the two clusters. The locations of the atoms corresponding to the number are given in fig. $4.35(0)[147]$. 
metal-sulfur bond and the metal-metal bonds are greatly weakened.

The above results represent the first step toward an understanding of grain boundary embrittlement in terms of changes in chemical bonds that must occur when an impurity atom resides at the grain boundary. To date, only the small embrittling elements in a limited number of clusters have been considered [148,149]. To have a complete theory, large atoms that may place a strain on the host atoms will have to be considered as well as many different cluster geometries. However, the concepts provided by these first calculations have delineated the central features of the electronic causes of embrittlement [147].

The above discussion brings forth certain important aspects relevent to this dissertation. First, it is theoretically possible to at least approximately identify the amount of interface enrichment and the resulting decrease in interfacial energy by having an estimate of the solid solubility of various impurities in the matrix. Segregation or presence of certain impurities, as has been discussed at length, leads to grain boundary decohesion and embrittlement. Sulfur and phosphorus have very low solid solubilities in austenitic alloys like Incoloy 903, and as such segregate to grain boundaries or if actually present at grain boundaries would prefer to remain there. In the case of welding, the constitutional liquation of MNP phosphides provides the requisite phosphorus enrichment of the liquid film. This excess phosphorus gets adsorbed at the solid-liquid interfaces. In areas slightly remote from the fusion line, the temperatures are conducive for grain boundary segregation of the impurities from the adjacent matrix regions during the weld thermal cycle. Hence, the constitutionally liquated regions and other grain boundaries in $H A Z$ where the temperatures allow for segregation of phosphorus and sulfur from the bulk are prone to liquid metal embrittlement as well as solid state embrittlement, when thermal and mechanical constraints act on them during the weld cooling cycle.

The embrittling capabilities of sulfur and phosphorus in nickel, iron and austenitic alloys will now be examined. There is evidence dating back to a century that nickel is 
extremely susceptible to embrittlement by trace impurities. Sulfur segregation to grain boundaries in nickel, nickel-base superalloys and austenitic stainless steels has been proved by Auger electron spectroscopy data. In austenitic stainless steels the elements $\mathrm{P}$ and $\mathrm{S}$ have been established as both promoting weld solidification cracking [150] and adversely affecting the mechanical properties. Many studies have shown a correlation between the $P$ and $S$ impurity level, the ferrite content and hot cracking $[151,152]$. It has also been observed that cracking did not occur when $P$ and $S$ level was less than $0.005 \%$ [153]. Both $\mathrm{P}$ and $\mathrm{S}$ exhibit rather low solubilities in iron. Because of the limited solid solubility of $P$ and $S$, as well as because of the small values of equilibrium distribution coefficients, these elements are strongly rejected into the interdendritic liquid during solidification. Microsegregation of both $\mathrm{P}$ and $\mathrm{S}$ has been reported at cell boundaries in austenitic stainless steels [154]. For both sulphide and phosphide inclusions, there is evidence that these were liquid at lower temperatures than the solidus temperatures of the matrix alloys. The phosphide films observed at austenite grain boundaries indicated extensive wetting.

Grain boundary liquation of nickel due to sulfur segregation is possible because the nickel-sulfur equilibrium phase diagram shows a deep eutectic trough on the nickel rich side. This fact, together with the observation that the minimum ductility trough in nickel containing free sulfur is near the $\mathrm{Ni}_{-} \mathrm{Ni}_{3} \mathrm{~S}_{2}$ eutectic temperature $\left(637^{\circ} \mathrm{C}\right)$, has prompted many investigators to attribute the embrittlement of nickel to the formation of liquid films of $\mathrm{Ni}_{3} \mathrm{~S}_{2}[155,156]$. Due to the retrograde solubility of sulfur in nickel, the formation of $\mathrm{Ni}_{3} \mathrm{~S}_{2}$ liquid films is quite plausible. Sulfur is also a very common and potent embrittler in iron and even at bulk concentrations of $10 \mathrm{ppm}$ by weight, one can find that significant amounts segregated on the grain boundaries. The solubility of $\mathrm{S}$ in $\gamma$ - $\mathrm{Fe}$ at $1356^{\circ} \mathrm{C}$ is only $0.05 \%$ [157]. It is also known that sulfur forms low melting eutectic with iron [157]. The Fe-rich eutectic temperatures in Fe-S diagram is consistent with this view [157]. However, the embrittling effect of free sulfur is generally controlled by additions of manganese and magnesium which tie up the free sulfur in the form of innocuous precipitates. 


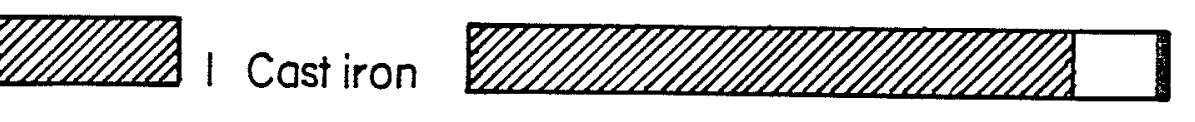

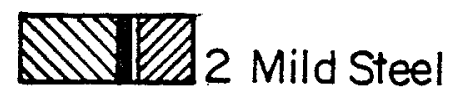
m. $\mathbb{N} 4{ }_{1} \mathrm{CrMOV}$

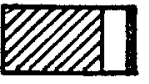
$53 \mathrm{Cr} / \mathrm{Mo}$

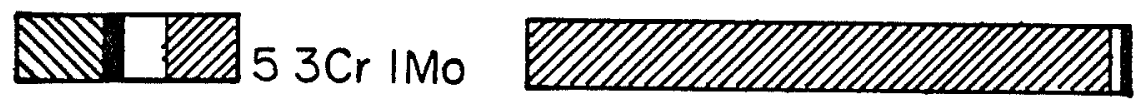

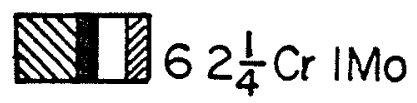
$\mathbb{Q}$. 75 NiCr-Mov

$\frac{1111111111}{0.10}$

Bulk Concentration, \%
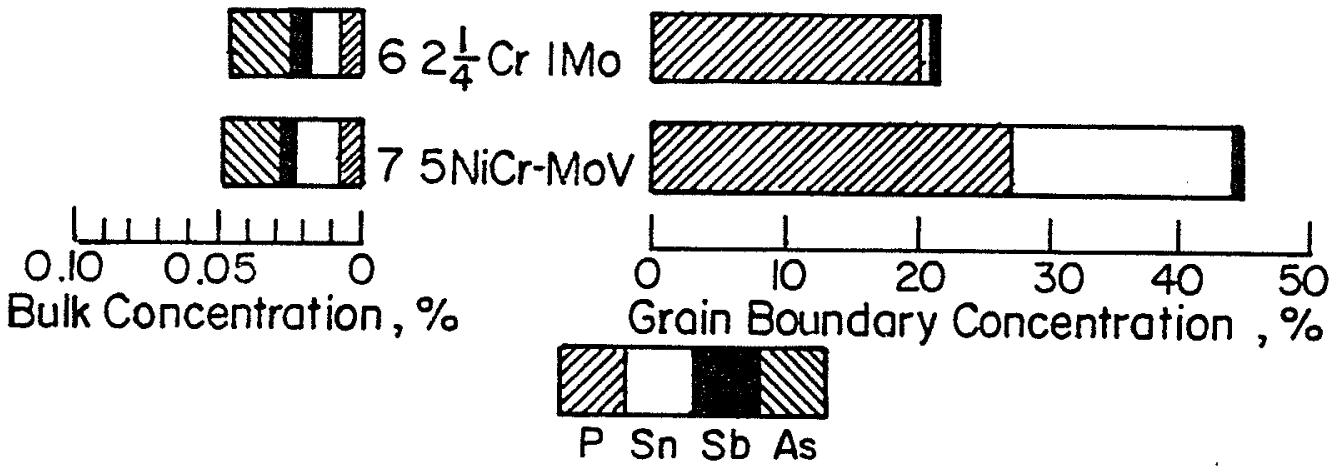

Fig. 4.36: Concentration of the four principal tramp elements at the groin boundaries of common commercial steels compared with the bulk content [158]. 
Phosphorus has also been observed to segregate at grain boundaries in iron and does cause embrittlement. Based on the studies from simple alloys, it appears that phosphorus is the most common embrittling element found on grain boundaries of commercial steels [147]. Fig 4.36 taken from the work of Hondros, Seah and Lea [158] shows segregation in several iron-base alloys for which concentration at grain boundaries has been measured and compared to the bulk concentration. As can be observed, phosphorus is the dominant segregating element in iron-base alloys containing phosphorus. Similarly, Larere et al. [159] have shown that sulphur and phosphorus segregate to free surfaces in nickel, whereas Park et al. [160] have shown grain boundary segregation of $P$ and $S$ in 304 stainless steel using Auger spectroscopy.

Since the solubility of phosphorus in austenite is even smaller than in ferrite, its equilibrium segregation should be correspondingly larger. The solubility of $\mathrm{P}$ in $\gamma$ - $\mathrm{Fe}$ at $1150^{\circ} \mathrm{C}$ is only $0.25 \%$. It is also known that $\mathrm{P}$ like $\mathrm{S}$ forms low melting eutectics with iron [157]. The Fe-rich eutectic temperature in the Fe-P equilibrium phase diagram is consistent with this view [157]. The results of Kaneko et al. [161] show that increasing the nickel and/or chromium content in austenite in steels reduces the solubility of phosphorus indicating a strong synergistic effect between the impurity and major alloying elements. An important feature of higher alloy fcc systems like austenitic stainless steels is that appreciable segregation of phosphorus can be acheived at high temperatures (solution treatments greater than $1000^{\circ} \mathrm{C}$ ) than in lower alloy steels [162]. This fact can be explained by Guttmann's cosegregation model leading to strong synergistic interaction between the impurity and the major alloying elements. The model suggests that increasing the alloying element content in more highly alloyed steels will drastically increase the impurity segregation.

In a system where the segregating element can also precipitate, there will be two opposing effects contributing to segregation. One of these will be the effect of temperature. If the bulk concentration of the segregating element does not change with temperature, 
the equilibrium grain boundary segregation will decrease with increasing temperature. This will be the only governing factor when the concentration of the element is below the solubility limit. Also, at a fixed temperature, an increase in the soluble bulk concentration will cause an increase in segregation and when the bulk concentration is above the solubility limit, these two effects will compete. An increase in temperature will decrease the driving force for segregation, but it will also increase the amount of segregating element in solution in the matrix which would tend to increase the amount of segregation. The relative magnitude of these two effects will determine whether an increase or decrease in segregation will occur with increasing temperature [163]. This aspect should be borne in mind when examining any data on segregation in superalloys where the residual elements like sulfur, phosphorus and boron have been observed to form precipitates.

The propensity for embrittlement depends on the amount of segregant, which in turn, is related to the bulk content of the species, and so the first procedure is to control the level of the damaging residual present in the bulk material. It is thus essential to identify the most potent embrittling species in a given system. In an investigation covering wide cross section of engineering steels, Hondros, Seah and Lea [158] showed that in general, phosphorus is the major embrittling agent in low temperature situations for low alloy steels of $\mathrm{Cr}$-Mo-V family, while in the group of the type Ni-Cr-Mo-V, both phosphorus and tin are important. Hondros and Seah [141] in their examination of grain boundary segregation have shown that enrichment factors of greater than about 100 lead to embrittlement.

Techniques for controlling segregation of trace elements to grain boundaries are critically important for a reliable mechanical, corrosion and welding performance in nickel-base superalloys [103]. In particular, elements such as phosphorus and sulfur are always deleterious both in austenitic stainless steels and superalloys, whereas boron (another melting point depressent [164]) may be deliberately added to the melt to improve creep rupture properties by interaction with other grain boundary segregants [165]. Vincent's [89] results on Inconel 718 weldments point towards boron being the most likely segregent active as 
a melting point depressent, although the possible synergistic interactions with other trace elements could not be excluded. Furthermore, it is also documented that boron forms low melting eutectic with both $\mathrm{Fe}$ and $\mathrm{Ni}$. Kelly $[59,166]$ based on statistically designed experimental heats of Inconel 718 concluded that boron is the single most detrimental element in terms of the weldability of superalloys.

The preceding discussion emphasized the role of tramp elements, like sulfur and phosphorus, in decreasing cohesion across grain boundaries/solid-liquid interfaces leading to grain boundary embrittlement based on electronic contributions, as well as their ability to decrease interfacial energy. Grain boundaries in HAZ will thus be embrittled if elemental phosphorus and sulfur are present on them as segregants and/or due to the solid state decomposition or constitutional liquation of phases that are rich in these elements. It has been discussed that these elements by decreasing solid-liquid interfacial energy increase wettability of any liquid that forms thus promoting grain boundary liquid metal penetration. The discussion also suggests that while the insiduous nature of sulfur can be controlled by additions of manganese or magnesium, there exists, as of now, no effective scavenger to offset the detrimental capability of phosphorus.

A relevent aspect that has not been addressed here in detail, owing to a lack of sophisticated experimentation, is the fact that a precipitate-matrix interface can be another potential site for segregation of tramp elements. In superalloys, the grain boundaries invariably contain carbides on boundaries and the preferential segregation of residual elements to carbide/matrix interfaces is a distinct possibility. The equilibrium thermodynamics and kinetics of segregation to these interfaces would be different than that of grain boundary segregation and segregation even at high temperatures to these interfaces cannot be ruled out. Evidence seems to exist for sulfur segregation to carbide interfaces in Inconel 718 given high temperature solution treatments [167]. This has been proposed as one of the causes for exacerbated microfissuring in Inconel 718 given high temperature solution treatments [167]. 
Table 4.11: List of effects of alloying and tramp elements on the weldability of nickel-base superalloys compiled by Pease [168].

\begin{tabular}{cccc}
\hline Beneficial & No Effect & Variable & Harmful \\
$\mathrm{Nb}$ & $\mathrm{Mn}$ & $\mathrm{Al}$ & $\mathrm{Pb}$ \\
$\mathrm{Mg}$ & $\mathrm{Cu}$ & $\mathrm{Ti}$ & $\mathrm{S}$ \\
& $\mathrm{Cr}$ & $\mathrm{C}$ & $\mathrm{P}$ \\
& $\mathrm{Fe}$ & $\mathrm{Mo}$ & $\mathrm{Zr}$ \\
& $\mathrm{Co}$ & $\mathrm{Si}$ & $\mathrm{B}$ \\
\end{tabular}


Pease [168] published a list of elements and their effects on weldability. This list, shown in table 4.11, indicates $\mathrm{Nb}$ to be beneficial (during that era, strain age cracking was the major problem with most superalloys and $\mathrm{Nb}$ additions were observed to decrease strain age cracking). The table also shows that boron (upper limit of $300 \mathrm{ppm}$ ) and other tramp elements such as sulfur and phoshorus to be harmful. About the same time, Owczarski $[1,16]$ and Shira [19] had determined that constitutional liquation of grain boundary NbC in the presence of various minor elements such as sulfur could cause microfissuring in the HAZ of superalloys. The work of Pease, Owczarski and Shira resulted in the current specification level of trace elements in nickel-base superalloys for acceptable weldability.

The effect of another grain boundary segregant, boron, needs special mention and discussion. Numerous experimental results exist on steels, superalloys and iron-nickel aluminides which suggest that if the bulk boron level is kept below a certain limit, boron increases the grain boundary cohesion. Hence, the preferential adsorption of boron to grain boundaries does not lead to solid state grain boundary embrittlement. Extrapolating these observations, it can be said that the preferential adsorption of boron to solid-liquid interfaces does not decrease the cohesive strength.

Boron is known [170] to slow the diffusion of carbon to the grain boundaries and delay the formation of $\gamma^{\prime}$-denuded zones which are believed [171] to play a critical role in strain age cracking (SAC/post weld heat treatment cracking (PWHT)). Furthermore, boron is well known to enhance the resistance to creep fracture, which is a process generally considered analogous to SAC. Thamburaj et al. [172] have observed a statistical correlation between SAC resistance and boron content. Higher levels of boron were found to correlate significantly with improved resistance to this type of failure. These results confirm that boron increases the grain boundary strength in solid state rather than leading to embrittlement.

In the context of fabrication weldability and hot cracking, phase diagrams show that boron like sulfur and phosphorus, forms a low temperature eutectic with nickel and iron. 
Hence, boron segregation to grain boundaries can cause grain boundary melting at temperatures below the bulk matrix temperature. Also, due to the presence of low temperature eutectic with the matrix, boron does increase the freezing range of the final terminal solidifying liquid on the HAZ boundaries. However, a study of documented experimental microstructural information shows no evidence of borides forming as terminal eutectic constituent or as continuous thin films on HAZ microfissures when boron levels are kept within the limits (specific to each alloy system). The following paragraphs critically review the published literature on the effect of boron on Inconel 718, a nickel-iron-chromium base superalloy. The discussion is primarily based on four studies, two by Kelly on cast alloys $[59,166]$, one by Vincent on wrought 718 [89] and another by Kelly [173] on Rene 220C (based on the $\mathrm{Ni}-\mathrm{Cr}$-Co system) a new cast superalloy developed for use in investment castings in the aerospace industry.

In an exhaustive study of wrought Inconel 718 weldments, Vincent had observed maximum number of $M_{3} B_{2}$ borides in $H A Z$ microfissures only in triple pass GTA welds (> 100 particles on a single replica) but in single pass GTA welds, the corresponding number of particles on a single replica were only in the range of only 5-10. The typical boride particle observed by Vincent was small (around $0.2 \mu \mathrm{m}$ ), and frequently composite with an irregular outline. Small MC carbides were observed to act as the central nucleus for borides. Vincent's speculation for the extensive occurrence of borides only in triple pass welds was that the fine carbides that did not dissolve during subsequent passes would become available for boride nucleation. In a sense, this speculation also amounts to preferential segregation of boron to carbide interfaces during the multipass welding cycle. The morphological evidence also suggests that the borides form not due to a eutectic reaction but more due to the precipitation as an intermetallic compound on the $H A Z$ boundaries within some temperature range during the HAZ cooling cycle. The absence of extensive coverage of HAZ boundaries by either borides or boride/boron films (since, Vincent had performed thin foil and EELS studies, if such films were to exist, they would 
have been easily detected) in single pass welds makes it difficult to attribute boron as being primarily responsible for microfissuring. Though the boron analysis of the samples investigated by Vincent was not reported, it was mentioned by him that the composition of the alloys were within the normal limits for alloy 718 .

Kelly [59] had used a statistical matrix which included $\mathrm{Fe}, \mathrm{Co}, \mathrm{Ta}, \mathrm{Si}, \mathrm{B}$ and $\mathrm{Mn}$ to prepare experimental castings similar to cast Inconel 718 and study the effect of these elements on the microfissuring susceptibility. He concluded that microfissuring varied almost directly with boron content. Kelly also suggested the possibility of some synergism between $B$ and $\mathrm{Fe}$, and came to the conclusion that $B$ at $100 \mathrm{ppm}$ level was the single most detrimental element. The chemical analysis of the experimental heats studied by Kelly is reproduced in Table 4.12.

Few interesting observations emerge on a closer examination of the compositions and total number of cracks observed in each of the individual heats. They are as follows: (i) While $B$ level in heats 7,10 and 12 are low $(0.0005 w t \%)$, all these heats contained only $0.05 w t \%$ Fe. It should be noted that:

(a) the absence of $\mathrm{Fe}$ would eliminate the possibility of the formation of low melting Laves phase in the base metal as well as on the HAZ grain boundaries (b) the composition of the above heats are similar to those of pure nickel-base superalloys and hence, as a first estimate, the weldability may be gauged from $\mathrm{Al}$ $\%$ vs $\mathrm{Ti} \%$ data on weldability shown in fig 2.21 .

(c) Only heat 10 is to an extent different, because it contains $3.08 \mathrm{wt} \% \mathrm{Ta}$. Since Ta is a strong carbide and a Laves phase former, the constitutional liquation of these phases during welding is a possibility. However, with respect to Ta, from the consideration of the phase diagram, it can be observed that tantalum additions can raise the Laves phase eutectic reaction temperature by as much as $77^{\circ} \mathrm{C}$ if complete substitution of $\mathrm{Ta}$ for $\mathrm{Nb}$ occurs. Hence, even if $\mathrm{Ta}$ is a Laves phase promoter, the total temperature range over which Laves phase liquid films exists 
Table $4.12[59]$

$\begin{array}{cccccccc}\text { Heat } & \text { No of } & \text { Co } & \mathrm{Fe} & \mathrm{Ta} & \mathrm{Si} & \mathrm{B} & \mathrm{Mn} \\ & \text { cracks } & & & & & & \\ 1 & 13 & 13.1 & 18.5 & 0.05 & 0.29 & 0.0048 & 0.21 \\ 2 & 14 & 12.9 & 0.05 & 2.99 & 0.28 & 0.0091 & 0.004 \\ 3 & 3 & 0.05 & 18.3 & 2.93 & 0.28 & 0.0005 & 0.004 \\ 4 & 2 & 12.9 & 18.4 & 3.03 & 0.04 & 0.0005 & 0.004 \\ 5 & 1 & 13.1 & 18.4 & 0.06 & 0.03 & 0.0005 & 0.2 \\ 6 & 25 & 13.0 & 0.05 & 0.03 & 0.05 & 0.006 & 0.004 \\ 7 & 0 & 0.05 & 0.05 & 0.07 & 0.27 & 0.0005 & 0.2 \\ 8 & 28 & 0.05 & 0.05 & 2.98 & 0.04 & 0.0127 & 0.2 \\ 9 & 43 & 0.05 & 18.2 & 0.2 & 0.29 & 0.0075 & 0.004 \\ 10 & 0 & 13.0 & 0.05 & 3.08 & 0.28 & 0.0005 & 0.2 \\ 11 & 36 & 0.05 & 18.1 & 3.11 & 0.05 & 0.0093 & 0.21 \\ 12 & 0 & 0.05 & 0.05 & 0.02 & 0.04 & 0.0005 & 0.004\end{array}$

The remaining elemental analysis was $19 \% \mathrm{Cr}, 5 \% \mathrm{Nb}, 3 \% \mathrm{Mo} 1 \% \mathrm{Ti}, 0.5 \% \mathrm{Al}$ and $0.04 \% \mathrm{C}$. Kelly did not report the compositions of sulfur and phosphorus in the alloys. 
in the HAZ is considerably reduced. Wlodek [174] has found that the stability of Laves phase is drastically reduced in the absence of Fe. Furthermore, the existence of large mismatch between $\mathrm{Ta}$ (at. no 73) and the gamma matrix, may promote the occurrence of grain boundary liquid film migration, if substantial local grain boundary curvature exists, eliminating or minimizing HAZ microfissuring.

(ii) Heat 9 which contained maximum number of cracks contained

(a) only 0.004 wt $\% \mathrm{Mn}$

(b) high amount of silicon (0.29 wt \%), 18.2 wt $\% \mathrm{Fe}$ and only $0.2 \mathrm{wt} \% \mathrm{Ta}$.

Factor (a) accentuates the effect of segregated sulfur due to the lack of scavenging action of $\mathrm{Mn}$ while factor (b) promotes extensive formation of low melting Laves phase.

(iii) Heats 1,2,6,8 and 11 which showed cracking can again be explained from physical metallurgical considerations.

(a) Heat 1 is prone to microfissuring because of the presence of $18.5 \mathrm{wt} \% \mathrm{Fe}$, $0.29 \mathrm{wt} \% \mathrm{Si}$ and only $0.5 \mathrm{wt} \% \mathrm{Ta}$ - all these factors promote the formation of low melting Laves phase eutectic.

(b) Heat 2 has boron content close to $100 \mathrm{ppm}$ and hence $M_{3} B_{2}$ borides may form and their subsequent liquation during welding is a possibility. Furthermore, a boron level of $100 \mathrm{ppm}$ may also cause grain boundary embrittlement. The decrease in the total number of cracks in heat 2 despite of it having a high boron content than heat 9 is illustrative of the fact that boron may not be the principal element causing microfissuring. The decrease in the total number of cracks in heat 2 is due to the presence of $\mathrm{Co}_{0}$ and $\mathrm{Ta}$.

(c) Heat 6 is expected to show microfissuring due to the low $\mathrm{Mn}$ content $(0.004$ $w t \%)$ and low $T a$ levels.

(d) Heat 8 has a boron level of $127 \mathrm{ppm}$ and Ta level of $2.98 \mathrm{wt} \%$ and the reasons for cracking advanced for the case of heat 2 apply. It should also be noted that heats 2 and 8 have very low Fe content making it only a nickel-base experimental 
alloy.

(e) Reasons for cracking advanced for the case of heat 2 apply to heat 11 . However, the more than two fold increase in total number of cracks in this heat is due to the presence of $18.1 \mathrm{wt} \% \mathrm{Fe}$. The high propensity to cracking of heat 11 as opposed to heat 8 should be noted. This again provides evidence that formation of low melting Laves eutectic increases the microfissuring susceptibility. Heat 11 has 18.1 wt $\%$ Fe while heat 8 has only 0.05 wt $\%$ Fe with the added presence of $2.98 w t \% \mathrm{Ta}$.

Heats 3,4 and 5 to an extent indicate that boron has some detrimental effect on the weldability of cast 718 . Kelly points out that the role of boron is to increase the wettability of the liquated Laves phase. However, in a cast structure containing Nb-rich phases, it is difficult to really homogenize the structure by using only thermal cycles due to the relative immobility of the larger Nb atoms in the Ni lattice; Radavich and Carlson [175] have shown that even though Laves phase can be solutioned into the matrix, and carbides reduced by heat treatment, the elemental segregation in the casting diminishes very little. This results in a microstructure that appears by optical microscopic examination homogeneous but is actually as segregated as the original casting and prone to the formation of low melting phases in some regions during the weld thermal cycle. The above discussion points to the fact that the formation of low meting $\mathrm{Nb}$-enriched films by eutectic reaction with the matrix is necessary and boron segregation by itself, without the presence of constitutionally liquatable regions, cannot increase the microfissuring susceptibility.

Kelly had undertaken another study on cast 718 [166] with a Plackett-Burman 20-run statistically designed matrix and investigated the first order effects of $\mathrm{C}, \mathrm{Si}, \mathrm{S}, \mathrm{P}, \mathrm{Nb}, \mathrm{B}$, $\mathrm{Fe}, \mathrm{Mo}, \mathrm{Hf}$ and $\mathrm{Zr}$ (the synergistic effects were not identified) as well as the effect of heat treatment. He again concluded that the primary cause of microfissuring in cast 718 is due to the boron content, followed by $\mathrm{Fe}$ and $\mathrm{Nb}$. A few comments are in order. The boron levels were chosen either to be at $100 \mathrm{ppm}$ or below $10 \mathrm{ppm}$. As suggested by 
the present author, boron levels higer than a critical amount may lead to the formation of low melting borides and may also embrittle $\mathrm{HAZ}$ grain boundaries due to extensive segregation. It should be noted that the results of Kelly's study [166] indicate that even a boron level as high as $0.01 \mathrm{wt} \%$ only increases the total crack length and the average crack length but had no statististically significant affect on the total number of cracks. Crack length itself or the total number of cracks appearing in a specific sample are not best suited as a measure of hot cracking susceptibility. One defines the extent to which an initiated crack is able to propagate; the other represents the probability of cracks appearing within a specific zone. Kelly's experimental result hence lends credence for the present suggestion that high boron levels embrittle the grain boundaries in HAZ thus making crack propagation easier. It is asserted, based on the reanalysis of Kelly's experimental results that boron does not have a significant role in the fundamental mechanism(s) causing HAZ microfissuring.

The nominal composition of the newly developed investment cast superalloy, Rene $220 \mathrm{C}$ and Inconel 718 is shown in table 4.13. As can be observed, the major difference lies in the complete elimination of $\mathrm{Fe}$ and $3 \mathrm{wt} \%$ addition of tantalum. The boron levels in alloy 718 and alloy $220 \mathrm{C}$ are the same and both alloys exhibit the same amount of segregation in the cast form. However, the elimination of $\mathrm{Fe}$ in $220 \mathrm{C}$ and addition of $\mathrm{Ta}$ eliminates the formation of low melting $(\mathrm{Fe}, \mathrm{Ni})_{2} \mathrm{Nb}$-type Laves phase eutectic. Wlodek [174] has observed the terminal solidification phase in $220 \mathrm{C}$ to be an unstable globular eutectic delta phase, which on heat treatment breaks down to form plates of delta phase. The plates of delta, however, have been observed to be stable up to the fusion boundary of the electron beam weldment. The stability of this phase arises from the increased $\mathrm{Ta}$ content of the alloy. Kelly [173] had observed the weldability of $220 \mathrm{C}$ to be superior to that of alloy 718 despite the presence of $30 \mathrm{ppm}$ of boron. From a study of various experimental heats, Kelly [173] has also reported that the weldability of $220 \mathrm{C}$ varies inversely with boron content in the range $30 \mathrm{ppm}$ to $100 \mathrm{ppm}$. However, the fact cannot 
Table 4.13: Chemical Composition of In 718 and Rene 220C [173]

\begin{tabular}{ccc}
\hline Element & Inconel 718 & Rene $220 \mathrm{C}$ \\
$\mathrm{Ni}$ & bal & bal \\
$\mathrm{Fe}$ & 18.0 & - \\
$\mathrm{Co}$ & - & 12.0 \\
$\mathrm{Cr}$ & 18.0 & 18.0 \\
$\mathrm{Nb}$ & 5.0 & 5.0 \\
$\mathrm{Ta}$ & - & 3.0 \\
$\mathrm{Mo}$ & 3.0 & 3.0 \\
$\mathrm{Ti}$ & 1.0 & 1.0 \\
$\mathrm{Al}$ & 0.05 & 0.05 \\
$\mathrm{C}$ & 0.05 & 0.03 \\
$\mathrm{~B}$ & 0.003 & 0.003
\end{tabular}


be ignored that the improved weldability of $220 \mathrm{C}$ with comparable levels of boron as compared with 718 has been primarily acheived through the elimination of low melting Laves eutectic by eliminating $\mathrm{Fe}$ and with the added addition of $\mathrm{Ta}$.

It should be noted that the weldability of Rene $41(0.01 \mathrm{wt} \% \mathrm{~B})$ is borderline and the weldability of M252 (B $0.005 w t \%$ ) is excellent. It should also be noted that though cast Inconel 718 is prone to microfissuring in the HAZ, in the wrought form of the alloy with comparable boron levels, microfissuring can be controlled by limiting the grain size to below ASTM 6 or finer by thermo-mechanical processing.

Brooks [40] had studied the effect of modification of the compositions of minor alloying elements on weldability of A286. He observed in Gleeble specimens a loss in on-heating ductility at $2100^{\circ} \mathrm{F}$ (approx. $1150^{\circ} \mathrm{C}$ ) that was determined to be directly related to the boron content. However, he could not detect any constitutional liquation of borides. The study of Brooks [40] also showed that a reduction in B content from 0.006 wt $\%$ to 0.001 wt \% greatly reduced the cracking tendency. However, a most recent review by Brooks and Thompson [154] on weldability of austenitic stainless steels contains no reference to the detrimental effect of boron. Residual element segregation (sulfur and phosphorus) to grain boundaries and subsequent embrittlement have been identified by them and many investigators as the generic cause for cracking in austenitic stainless steels as opposed to boron segregation.

David et al. [176] have investigated the effect of boron on the weldability of iron-nickel aluminides. They observed the severity of $\mathrm{HAZ}$ microfissuring to dramitically decrease with additions of boron upto a level of $200 \mathrm{ppm}$ and then increase with further additions of boron. Boron additions in controlled amount is known to increase grain boundary cohesion in $\mathrm{Ni}_{3} \mathrm{Al}$. Although boron segregates preferentially to grain boundaries in boron doped aluminides, no localized melting was observed in the $\mathrm{HAZ}$ of the aluminides. The results of David et al. [176] show that boron additions in controlled proportions are beneficial to weldability. Another interesting aspect that should be noted is the total absence of sulfur 
and phosphorus in their material.

While studies based on statistical design are useful in providing an idea as to the first order effects of different elements on weldability, the results should never be taken apriori without a careful examination of the physical metallurgical changes that can be brought about by minor alterations in alloy chemistry. While studies by Kelly show boron as the most detrimental element to weldability above a certain range, no attempt was made in any of the studies to examine the different microstructures by AEM and whether any borides form and/or boron substitution occurs in the phases that exist. It should again be emphasized that in the studies by Kelly, boron showed a statistically significant effect on cast 718 weldability only in the measurement of total and average crack length but had no effect on the total number of cracks. Furthermore, Kelly has found that as the heat treatment temperature and time increases, the response of many of the variables also increases. As reported, if segregation of elemental boron to grain boundaries (assuming no borides form) is responsible for boundary melting, then the increased microfissuring susceptibility to high temperature solution treatment cannot be explained. If non-equilibrium segregation of boron during cooling from the high temperature were to be evoked as a possible mechanism, then the increased microfissuring tendency with increase in aging time at a constant temperature observed by Kelly [166] cannot be logically rationalized.

Statistical studies of the type performed by Kelly $[59,166]$ consider only the first order effects of various elements and ignore all the different synergistic effects. However, synergistic effects play an important role in determining the kinetics and extent of segregation in multicomponent systems. Evidence abounds in literature on segregation in multicomponent systems which suggests that Guttmann cosegregation can predict the trend of the possible synergistic effects. For example, the alloying additions of $\mathrm{Ni}-\mathrm{Cr}-\mathrm{Mn}$ alone or in association with plain carbon steels has been shown to enhance the equilibrium segregation potency of metalloids $(P, S b, S n)$ by a factor of 5 to 10 [162].

Hence, a critical look at the available experimental evidence suggests that the role 
of boron segregation being primarily responsible for $\mathrm{HAZ}$ microfissuring can at best be termed as only ambiguous. While boron does form low melting eutectic with both Fe and $\mathrm{Ni}$, no concrete evidence of this eutectic as one of the terminal solidification constituent spread continuously along $H A Z$ microfissures has been documented in literature. In all other studies where low melting eutectic terminal solification contituents were responsible for HAZ microfissuring, considerable experimental evidence exists for the presence of these phases on HAZ microfissures. Also, the embrittlement effects of elements like sulfur and phosphorus have been extensively verified in austenitic stainless steels. In the present investigation, no $M_{3} B_{2}$ boride films were observed in the phases extracted from the microfissures. As the above discussion shows, there exists no reliable evidence to pin boron as the most single detrimental segregant causing $\mathrm{HAZ}$ microfissuring.

It is also to be noted however, that because of the low mutual solid solubility of $B, P$ and $S$ in the gamma matrix, equilibrium segregation of these elements to grain boundaries can be significant to cause sufficient damage even during high temperature solution treatments. The preceding discussion has also shown that $S$ and $P$ are more detrimental tramp elements to weldability than boron. However, the author concludes the discussion on boron effect by stating that the effect needs careful study on experimental heats which are (i) low in $P$ and $S$ and (ii) whose bulk boron content is within the known acceptable levels where increased grain boundary cohesion and strengthening are achieved.

The boron effect is a perplexing problem to a material scientist. The exact reasons as to the mechanism by which boron strengthens the grain boundaries and the reason for deleterious effects if bulk boron levels exceed a certain level are still embroiled in controversy. Some progress has been made in understanding the role of boron in increasing grain boundary cohesion by the consideration of electronic factors [147]. While this dissertation does allude to the possibility of some detrimental effect of boron in the subsequent discussion on HAZ microfissuring in Incoloy 903, it does not pin point boron as the most detrimental element. The addition of cobalt and the decrease in niobium level eliminate 
the formation of Laves phase in Incoloy 903 . Incoloy 903 is closely related to austenitic stainless steels and does contain $70 \mathrm{ppm}$ boron. But previous studies by Baeslack and coworkers [79] did not identify a correlation between boron and a loss in ductility in Gleeble hot ductility testing. Borides, per se, were not observed to precipitate in this study though $B$ could substitute for $P$ in MNP phosphides, if final solution treatment temperature after thermo-mechanical processing is kept below $950^{\circ} \mathrm{C}$. Furthermore, no formation of borides or thin boride films was observed in any of the HAZ microfissures.

Before concluding the discussion on the section of impurity segregation and embrittlement it would be in order to discuss in brief the phenomenon of liquid metal embrittlement (LME) because LME could be one of the mechanisms responsible for $\mathrm{HAZ}$ microfissuring. LME is the reduction in the elongation to failure that can occur when normally ductile metals or alloys are stressed while in contact with liquid metals. LME failures occur by the nucleation of a crack at the wetted surface of the solid and its subsequent propagation into the bulk. There are two necessary but not sufficient conditions that must be satisfied simultaneously for LME to occur;

(i) The applied stress should be sufficient to produce plastic deformation

(ii) there should be direct contact on an atomic scale between the stressed solid and the embrittler which implies that the liquid must flow into any crack which may form and propagate $[177,178]$.

LME failure is usually intergranular and hence it is to be expected that variations in grain size, microstructure, or the grain boundary chemistry should influence the susceptibility of stressed solids. Whether or not LME occurs on a particular occasion depends not only on the composition of the solid/liquid combination but also upon the metallurgical state of the solid and the exposure conditions. One of the interesting aspects of LME is its apparent specificity; i.e., certain liquid metals can embrittle only certain solid metals. Few emperical rules have been advanced to guess possible embrittling systems:

(i) if the two metals involved form stable high melting intermetallic compounds 
in the solid state, then its unlikely that they constitute an embrittling couple (ii) embrittlement rarely occurs in systems in which two metals exhibit mutual solubility. Hence, the occurrence of LME must be determined by the chemicalelectronic interactions between the solid and the liquid and the associated reduction in cohesion [178].

The mechanisms that have been proposed for LME are

(i) reduction in suface energy model-i.e., a reduction in the surface free energy of the solid metal by the adsorbing species is related to the surface tension between the solid and the liquid metal,

(ii) adsorption-induced reduction in cohesion model-embrittlement is associated with a localized reduction in the strength of the atomic bonds at the crack tip or at the surface of the solid metal by certain chemisorped species,

(iii) stress-assisted dissolution model-where the crack is asssumed to propagate by the solution of the solid in the liquid under the influence of an applied stress, with volume diffusion of the dissolved solid through the liquid controlling the propagation.

Kamdar [178] in his review concludes that the stress-assisted dissolution model is an unlikely possibilty. He also reasons that the reduction in surface energy is obvious since embrittlement effects must originate at the solid-liquid metal interface and hence energy considerations are important. However, he points out that the experimentally determined values of total energy involved in crack propagation are several orders of magnitude greater than the surface energy of the solid. Furthermore, the model does not provide insights into the mechanism of embrittlement on an atomic or electronic scale. Westwood and Kamdar's [179] experimental work does provide evidence for the reduction in cohesion strength model for LME.

The concept of an 'inert carrier' liquid metal can be used to induce and control the severity of LME. Small as well as large variations in the composition of the liquid can 
cause significant variations in the severity of embrittlement. Minor alloying additions to the embrittling liquid metal have been shown to either reduce or increase the severity of LME [178]. However, the role of the liquid metal and its solutions in controlling the severity of embrittlement and the associated mechanism(s) are not well understood. 


\section{6: MECHANISM(S) FOR HAZ MICROFISSURING}

HAZ microfissuring is generally considered to be caused by the presence of low melting liquid phases on the grain boundaries. The liquid enables the boundaries to separate under the thermal and shrinkage stresses, and mechanical constraints during the weld cooling cycle while the phases are still above the solidus temperature. Several mechanisms have been proposed to explain HAZ liquation cracking, the most widely accepted being the grain boundary penetration mechanism wherein a migrating grain boundary interacts with liquating matrix particles like carbides, sulphides, borides, etc.. HAZ grain boundary liquation, in the absence of liquating species on the grain boundary, requires the simultaneous occurrence of constitutional liquation and grain boundary migration. The penetration of the boundary by the liquid at the particle-matrix interface either pins the boundary or significantly slows its rate of migration and allows uniform wetting of the boundary by the liquid. These liquid films serve to embrittle the HAZ grain boundaries in the presence of mechanical and/or thermally induced restraint imposed during the welding leading to HAZ cracking.

HAZ liquation cracking has been explained in a wide variety of nickel-base superalloys as occurring due to the constitutional liquation of $M C$ and $M_{6} C$ carbides $[1,16]$. In an investigation of the phase transformations in simulated $H A Z$ in alloy 800 , Romig et al. [48] have reported that the submicron $\operatorname{Ti}(C, N)$ particles already present on the grain boundaries liquates in a eutectic-type reaction with the surrounding matrix. HAZ grain boundary liquation cracking has also been reported in maraging steels [46] and in austenitic stainless steels [40].

Another mechanism that has been used to explain HAZ liquation cracking is the grain boundary segregation mechanism which emphasises the local lowering of the melting point and consequent liquation due to the segregation of certain elements to the grain boundary. Equilibrium elemental segregation as well as non-equilibrium segregation due to a sweeping mechanism of elements that locally lower the melting point at the grain 
boundaries has been proposed to explain HAZ liquation cracking in materials that do not possess liquating particles. A pipe line diffusion theory, whereby the solutes partitioned into the interdendritic liquid in the fusion zone diffusing along contiguous grain boundaries in the HAZ has also been utilized to explain the HAZ cracking occurring adjacent to the fusion line.

As discussed in chapter 2, not all theories of hot cracking propose that fracture occurs due to the rupture of thin liquid films. The theory of Pumphrey and Jennings [22] holds that it is not the liquid film that ruptures, rather, it is the isolated solid bridges between cells or dendrites that fail. Be it the liquid film or the solid bridges that rupture, all the models ascribe hot cracking to what goes on in the very last stages of the terminal solidification between cells or dendrites, when liquid metal cannot flow into the cracks and heal them, provided the liquid does not contibute to LME failure. If the liquid is capable of inducing an LME failure, microfissuring is exacerbated.

In the forged and solution treated ring of Incoloy 903, the grain boundaries prior to welding were decorated with MNP phosphides $(0.1-0.5 \mu \mathrm{m}$ in size) and fine carbides (50 $\AA-200 \AA$ in size). In the as-forged samples solution treated for an hour at $975^{\circ} \mathrm{C}$, fine carbides ( $50 \AA-200 \AA$ in size) were present on the grain boundaries. In the forged and solution treated ring samples further solution treated for varying times at $1100^{\circ} \mathrm{C}$, coarsened fine carbides were observed on the grain boundaries. In all the microstructural conditions, some primary carbides/carbonitrides also existed both on the grain boundaries and in areas adjacent to them. It has been shown in earlier sections that phosphides go into solution around $975^{\circ} \mathrm{C}$. However, due to the relatively large size of MNP phosphides, complete solid state dissolution may not be possible during the rapid heating cycle of electron beam welding. Instead, constitutional liquation of phosphides, fine carbides and primary carbides (partial) is observed to occur.

The microstructural evidence presented in the previous sections indicate the presence of niobium-enriched liquid on the $H A Z$ grain boundaries. The liquid formed on the $H A Z$ 
grain boundaries in Incoloy 903 weldments appears primarily to originate from the constitutional liquation of prior precipitates on the boundary. A part of the liquid may also have possibly originated due to grain boundary segregation caused by grain boundary mobility or sweeping, a phenomenon schematically illustrated in fig 4.37. In the HAZ, the extent of grain boundary mobility is primarily dependent on the distribution of intermetallic and secondary phases and the metallurgical nature of the base metal. The grain boundaries in the base metal of Incoloy 903 consists of primary carbides and other trace phases. As these particles constitutionally liquate and/or dissolve during the weld heating cycle, grain growth occurs and the remnant impurity elements may be swept into the boundary. Alternately, the migration of the grain boundaries and subsequent intersection with constitutionally liquated primary carbides assure the occurrence of grain boundary penetration of the niobium-enriched liquid. Hence, the occurrence of constitutional liquation of preexisting precipitates as well as some grain boundary penetration of the liquid formed by the constitutional liquation of primary carbides lying in the matrix adjacent to the boundary, leads to the formation of low melting liquid films on the HAZ grain boundaries enriched in

(i) niobium, titanium, carbon, phosphorus and boron in the case of samples solutionized below $950^{\circ} \mathrm{C}$ and

(ii) primarily niobium, titanium and carbon in the case of thermo-mechanically processed samples given final solution treatments above $950^{\circ} \mathrm{C}$.

In the solution treatment temperature ranges where extensive precipitation of MNP phosphides occurs $\left(800^{\circ} \mathrm{C}-925^{\circ} \mathrm{C}\right)$, even if substantial amounts of boron were not to be tied up in the phosphides, extensive equilibrium segregation of boron and sulfur to grain boundaries occurs during thermo-mechanical processing and final solution treatment. Even in the case of high temperature solution treatments, segregation of sulfur, boron and phosphorus may occur because of their very low solid solubility in the gamma matrix. As discussed earlier, the addition of nickel to plain carbon steels, i.e., in high alloy steels, 

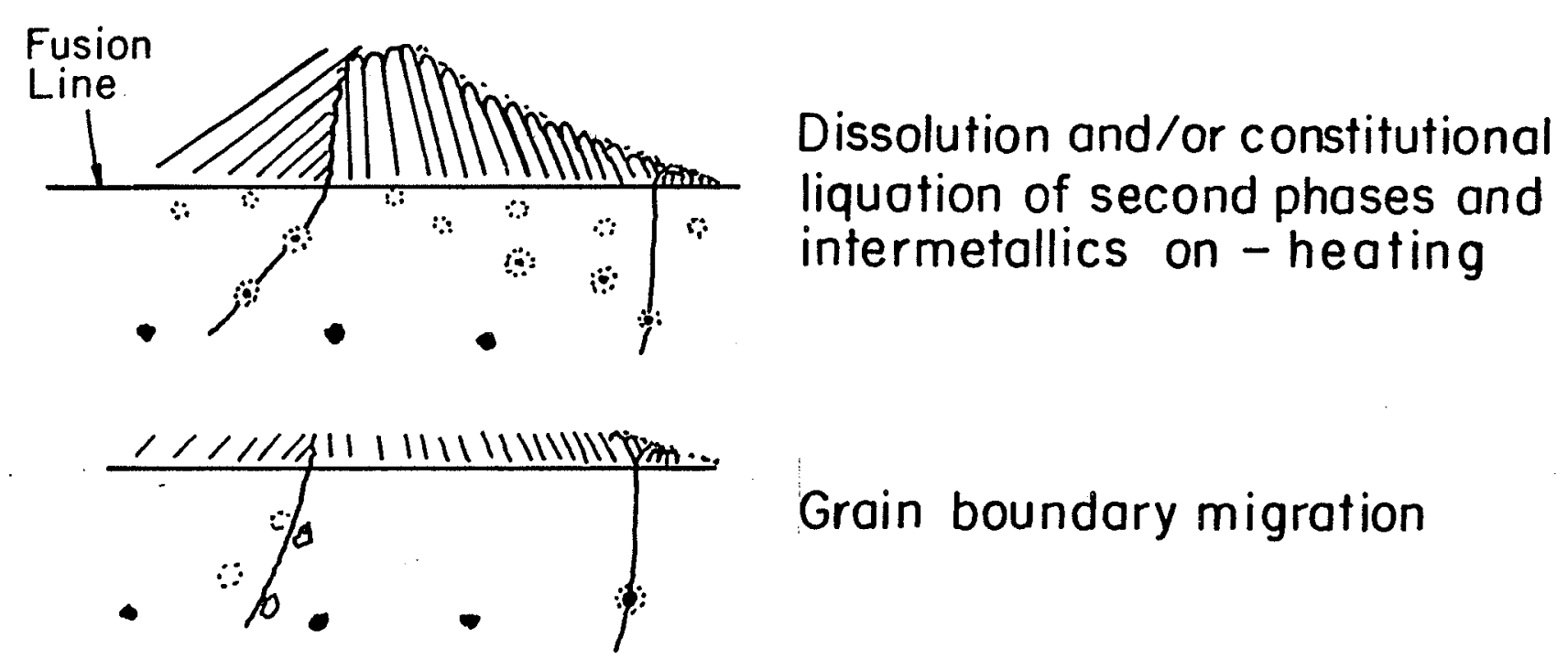

Grain boundary migration

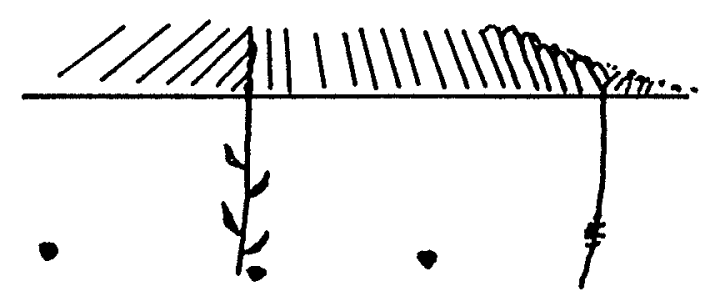

Assimilation of melting point depressant elements

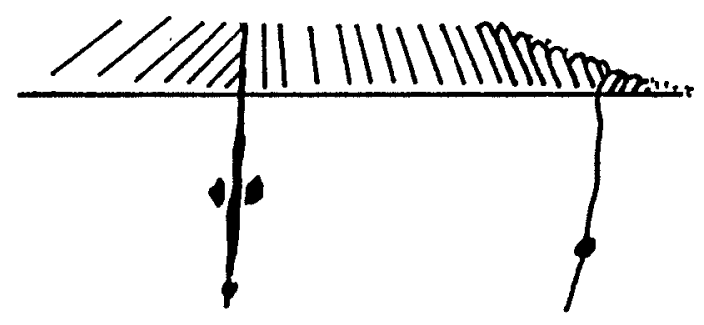

Boundary melting

Fig. 4.37 : Schematic illustration of grain boundary sweeping in the weld HAZ. 
the solubility of phosphorus in the gamma matrix is considerably reduced. The potency for grain boundary enrichment is also enhanced by a factor of 5 to 10 in the presence of nickel. Also, considerable equilibrium segregation occurs even at high temperatures (greater than $1000^{\circ} \mathrm{C}$ ) [162].

Extensive liquation and microfissuring is also observed in regions slightly removed from the fusion line which also demonstrates local melting at the grain boundaries. Fig 4.38 shows the far heat affected zone with an intergranular crack running along a grain boundary containing niobium rich carbides and phosphides. A similar observation has been reported for A286 [40], Incoloy 903 [77], and Inconel 718 [42]. In all these alloys, cracking was most prevalent in the region of the $H A Z$ slightly removed from the fusion line.

Several possible explanations were offered [21] for the occurrence of cracking in the region of $H A Z$ remote from the fusion line. They are:

1. The crack susceptible region is sufficiently remote from the weld pool that liquid backfilling of cracks is not possible.

2.Grain boundary liquation occurs on a more localized scale than is apparent with optical microscopy and, in fact, all boundaries are liquated.

3. Liquation and subsequent cracking, only occurs along boundaries that have intersected a constitutionally liquated particle.

4. Localized grain boundary strains are much higher in regions remote from the fusion line than in the mushy zone adjacent to the fusion zone.

5. Crack initiation may occur in the heavily constitutionally liquated regions with propagation proceeding via a liquid metal embrittlement mechanism along the grain boundaries.

The occurrence of microfissures slightly remote from the fusion line may be due to the fact that crack initiation may occur in the heavily constitutionally liquated regions with propagation proceeding via a liquid metal embrittlement mechanism along the grain boundaries. It is also likely that once a crack is initiated, it propagates easily along the embrittled grain boundaries in $H A Z$, the embrittlement being caused by the presence of 


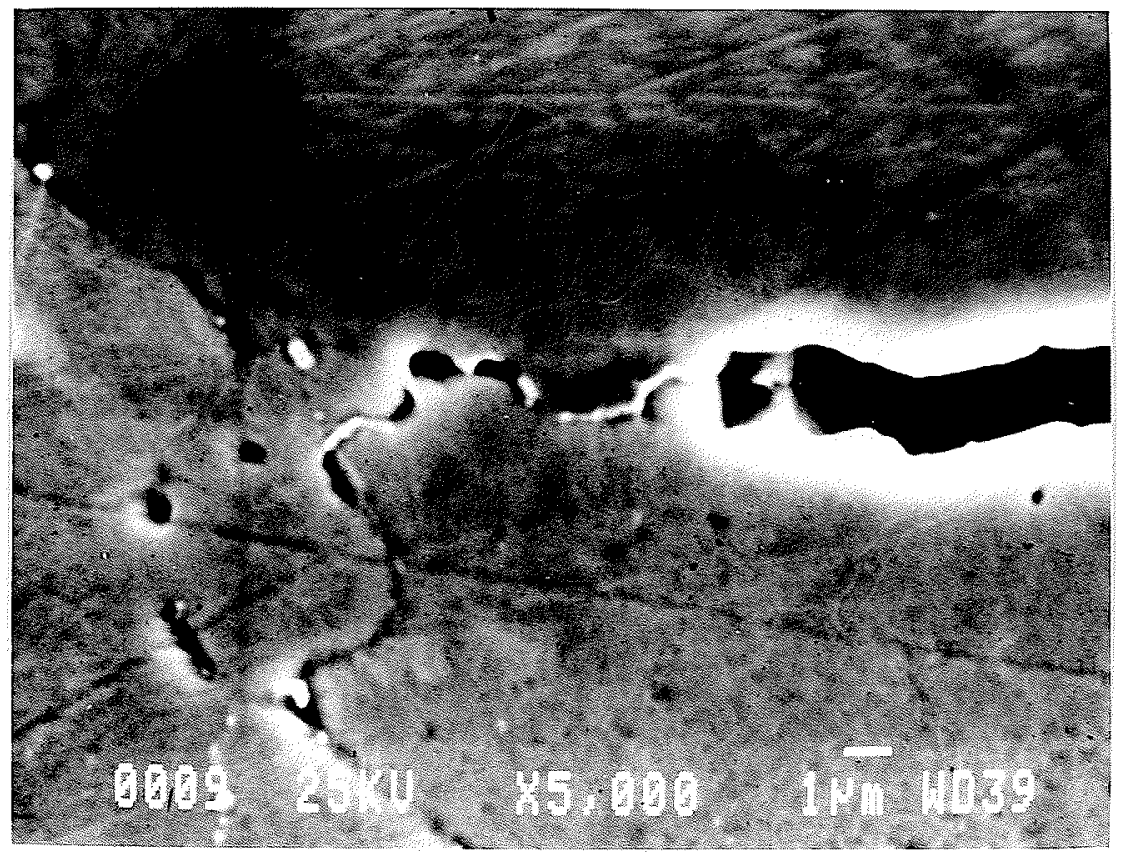

fig 4.38: SEM micrograph showing HAZ cracking remote from the fusion line. 
tramp elements like sulfur and phosphorus. This seems the most plausible explanation from the observations in this study as HAZ microfissures were observed to be extending partly into the fusion zone suggesting that a HAZ microfissure could propagate in either direction during the liquid film stage or in solid state along embrittled grain boundaries.

The composition and thickness of the liquid films formed on the HAZ grain boundaries due to liquation is dictated by

(i) the relative proportion of the volume fraction of phosphides and carbides undergoing constitutional liquation,

(ii) the amount of segregation that had earlier occurred at the grain boundaries during thermomechanical processing, solution treatment and during welding,

(iii) the amount of liquid metal penetration occurring from constitutionally liquated primary carbides lying close to the HAZ grain boundaries.

The phosphorus and boron concentration is maximum in the liquid films formed during electron beam welding in the as-forged alloy and in the forged and solution treated ring. In the as-forged alloy solution treated at $975^{\circ} \mathrm{C}$ for an hour and in samples of the forged and solution treated ring further solution treated for varying times at $1100^{\circ} \mathrm{C}$, the liquid films are primarily enriched in niobium, titanium and carbon. Since, no volume fraction measurements were undertaken, the present investigation cannot provide a quantitative answer to the thickness of the liquid films formed in the electron beam welded samples with different initial base metal microstructural conditions. However, this dissertation does address qualitatively the nature and thickness of the liquid films formed on the HAZ grain boundaries while comparing the microfissuring susceptibility.

It should however, be pointed out that precise volume fraction measurements in this investigation were prone to error due to

(i) the large grain size in certain microstructural conditions and

(ii) the artifacts involved with the preparation of carbon extraction replica and subsequent TEM examination. 
Thin foil studies would have provided a more definitive answer. However, the author's experience indicates the Herculian task of obtaining thin foils of the HAZ even in the cases where minimal microfissuring occurred; electropolishing or ion beam milling provide no solution in obtaining selectively transparent areas of a microfissure. With secondary ion mass spectroscopy, one can to a better approximation determine the volume fraction of the solidification constituents that form on the HAZ grain boundaries.

The metastable liquid film formed on the grain boundaries has two ways of relieving its supersaturation which is governed by the composition of the liquid and the solid-liquid interfacial curvature; either by the migration of the liquid film into the matrix leaving behind in its wake an alloyed zone or by normal solidification and formation of dendritic and interdendritic constituents. The dominant solidification mode is controlled by the composition of the liquid and the solid-liquid interfacial curvature. The driving forces and the kinetics of LFM were discussed at length in the earlier sections. LFM is favored if large coherency strains and substantial interfacial curvature exists. With the occurrence of LFM, the supersaturation in the liquid is relieved faster and the total temperature range of solidification is greatly reduced, thus decreasing the possibility of grain boundary microfissuring. It is emphasized again that the thickness and composition of the liquid film also play a major role over the temperature range in which the supersaturation is relieved; for a fixed composition a critical thickness exists, above which even the occurrence of LFM will not be able to preclude the occurrence of terminal eutectic reaction(s).

It was also discussed at length and concluded that initiation of LFM on large straight warm worked boundaries in forged and solution treated ring is extremely difficult and as such the liquid formed on the grain boundaries is forced to relieve its supersaturation by normal solidification. With the formation and growth of gamma dendrites, solutes with partition coefficient less than unity are rejected into the interdendritic liquid. These solutes determine the nature of the interdendritic liquid and the total solidification temperature range. The greater the range of solidification, the greater is the susceptibility for grain 
boundary microfissuring. In Incoloy 903 , most of the normal solidification terminates with the gamma/carbide monovariant eutectic reaction. Solutes like phosphorus, silicon, sulfur and boron have extremely low value of partition coefficient and are, hence, extensively rejected into the interdendritic liquid along with niobium, titanium and carbon. The effect of tramp elements like phosphorus, sulfur and boron in reducing solid-liquid interfacial energy and in causing solid state embrittlement of $\mathrm{HAZ}$ grain boundaries was discussed in earlier sections. During the final stages of solidification, if thin continuous and/or semicontinuous films exist on the HAZ grain boundaries, HAZ microfissuring occurs if sufficient thermal and mechanical constraints exist. The existence of thin continuous liquid films is partly governed by the solid-liquid interfacial energy considerations. The liquid on HAZ grain boundaries can spread easily if it extensively wets the grain boundaries.

Evidence exists in the literature [36] that the liquid formed by the constitutional liquation of $\mathrm{NbC}$ does wet the grain boundaries. Further evidence to the fact that the liquid formed by constitutional liquation of primary carbides and fine carbides in Incoloy 903 wets the grain boundaries is provided by the occurrence of LFM in case A welds of asforged samples solution treated for one hour at $975^{\circ} \mathrm{C}$. LFM can occur only in cases where solid-liquid interfaces are in initimate contact. As can be observed from the micrographs presented in section 4.3.3, extensive LFM occurs all along the liquated grain boundaries in the HAZ rather than at discrete pockets. The reason as to the occurrence of minimal microfissuring on the fine recrystallized grains in the forged and solution treated ring and in samples of as-forged alloy solution treated for an hour at $975^{\circ} \mathrm{C}$, as opposed to the long, straight warm worked grain was discussed in section 4.4 and was demonstrated to be related to the mean curvature of the grain boundaries.

Another factor that has been identified in austenitic stainless steels to be important in causing $H A Z$ grain boundary microfissuring is the nature of the grain boundaries. It has been shown by Brooks and Thompson [154] that a less tortuous grain boundary path is more susceptible to microfissuring as opposed to a tortuous grain boundary path. 
A less tortuous grain boundary path facilitates easier spreading of the liquid as well as it makes both crack initiation and crack propagation easier. Long warm worked and large equilibrated grain boundaries provide an easier path for the spreading of the liquid films and crack propagation. The inability of the long, straight HAZ grain boundaries to withstand microfissuring is due to the fact that a straight boundary leads to a thinner liquid film, provided the liquid extensively wets the boundary. Furthermore, the ability of long straight boundaries perpendicular to the fusion line to withstand the thermal and mechanical constraints is limited as compared to small volumes of solid connected by continuous liquid films.

Hence, the role of tramp elements like sulfur and phosphorus in exacerbating microfissuring susceptibility appears to originate from their

(i) ability to decrease the solid-liquid interfacial energy further facilitating easy spreading of the liquid along HAZ grain boundaries,

(ii) ability to decrease the solid-liquid cohesion leading to easy parting across one of the solid-liquid interfaces (i.e., causing easy crack initiation),

(iii) ability to increase the total time range over which solidification occurs due to their ability to form low melting eutectics with the gamma matrix, (iv) ability to embrittle the HAZ grain boundaries in solid state by decreasing the grain boundary cohesion,

(v) ability to decrease the driving force due to coherency strain energy by decreasing the total coherency strain in the thin volume diffusion zone in the solid at the solid-liquid interface.

The first three factors make the process of crack initiation under thermal and mechanical constraints easier; factor (iv) promotes crack propagation even in the solid state under thermal and mechanical constraints. It should also be noted that extensive segregation and/or the presence of boron on HAZ grain boundaries accentuates the role of factors (i),(ii) and (iii). Factor (v) decreases or may even completely eliminate the occurrence of 
grain boundary LFM. However, boron segregation below a certain limit that is specific to each alloy system does not cause solid state embrittlement; on the other hand, it leads to increased grain boundary cohesion.

It has been reported in many investigations $[63,169]$ that fine grain sizes reduce the HAZ microfissuring susceptibility. In wrought Inconel 718 , microfissuring is considerably minimized if the grain size is kept below ASTM \# 6. Thompson et al. have observed a linear relationship between microfissuring susceptibility measured in terms of TCL and grain size in wrought Inconel 718 [63]. Morrison et al. [169], however, found no correlation between grain size variance and microfissuring for grain sizes less than ASTM \# 2 . Thompson et al. [63] have suggested that the shear component of stress accentuated the microfissuring. They attributed this to a grain boundary sliding mechanism of microfissure initiation. They suggested that large grain sizes promote grain boundary sliding stress.

In the present investigation, various grain sizes were generated in the range of $60 \mu \mathrm{m}$ to $200 \mu \mathrm{m}$ by solution treating the forged and solution treated ring for $1 / 2 \mathrm{~h}, 1 \mathrm{~h}, 2 \mathrm{~h}, 4 \mathrm{~h}$, and $8 \mathrm{~h}$ at $1100^{\circ} \mathrm{C}$. Extensive $\mathrm{HAZ}$ microfissuring occurred under all these conditions; however, the increase in TCL with grain size is likely. The present suggestion is that coarse grain sizes provide a less tortuous path for crack propagation as opposed to fine grain sizes which leads to increased TCL and maximum crack length. However, looking at figs 4.15, $4.17,4.20-4.22$, it is evident that the grain sizes in the range investigated does not alter the microfissuring susceptibility or the mechanism of microfissuring.

It is likely that extremely fine grain sizes may reduce the HAZ microfissuring susceptibilty, but it may not be the grain size per se that reduces microfissuring. It is more likely that the microfissuring is reduced due to the existence of substantial mean grain boundary curvature. The greater the mean grain boundary curvature, the greater is the driving force available for the possible occurrence of LFM on the liquated boundaries, provided sufficient coherency strains exist in the thin volume diffusion zone. Evidence exists that extensive LFM does occur in Inconel 718 . This is because, the grain boundary liquid 
in Inconel 718 is also enriched in niobium. Unfortunately, no grain boundary curvature measurements were undertaken in any of the above mentioned experimental studies on the effect of grain size on HAZ microfissuring susceptibility.

Recently, Patterson and Liu [137] have experimentally determined the relationship between mean grain boundary curvature and the mean grain size. They observed the mean grain boundary curvature to be linearly related to the inverse of mean grain intercept. The value of the ratio of average mean grain boundary curvature to the inverse of mean grain intercept was found to be 0.31 for pure aluminum throughout the grain growth regime. Patterson and Liu's work provides experimental evidence to the present assertion that a fine grained structure possesses substantial mean grain boundary curvature, and hence the occurrence of LFM and consequent minimization of HAZ microfissuring is likely.

The results of the present investigation provide definitive evidence to the fact that it is the mean grain boundary curvature that is more important than the mean grain size if there exists sufficient atomic mismatch between the dominant solute in the liquid and the matrix. The grain size of the as-forged alloy solution treated for an hour at $975^{\circ} \mathrm{C}$ is comparable to the grain size of the forged and solution treated ring further solution treated for half an hour at $1100^{\circ} \mathrm{C}$. Substantial local mean grain boundary curvatures exist only in the former solution treatment due to the pinning of grain boundaries by fine carbides. Microfissuring was observed to be minimal in the former case as opposed to extensive microfissuring in the latter.

It has been pointed out in many studies on fusion zone and HAZ microfissuring that the mere occurrence of a liquation reaction, either by constitutional liquation or boundary segregation induced liquation, is not sufficient to produce a crack susceptible microstructure. The emphasis, however, has been that it is necessary that the liquid species penetrate and wet the grain boundary. If the wetting is ineffective, liquid will exist in isolated pockets allowing substantial solid-solid contact along the grain boundaries. This is ofcourse, apart from the fact that liquid films should persist until a time where 
sufficient thermal and mechanical restraints exist. This implies that a large temperature range of solidification is essential.

Later, Cieslack [117] has shown that in alloy 625 even the existence of a large freezing range may not necessarily lead to microfissuring. The present investigation identifies another important factor that is essential - the existence of grain boundaries with insignificant mean curvature if the solutes in the liquid possess a large mismatch with the matrix atoms; this is because existence of large mismatch and presence of significant mean grain boundary curvature facilitates the occurrence of LFM.

The results of the present investigation do corroborate the fact that it is essential for a continuous and/or semicontinuous film of liquid to exist on HAZ grain boundaries for cracking to occur under the presence of thermal and mechanical restraints and that cracking would not occur if liquation occurs only in isolated pockets on the grain boundary. Once a crack is initiated, it can propagate in the solid state if HAZ grain boundaries are embrittled and provide a less tortuous path. However, the existence of continuous/semicontinuous liquid films is not a sufficient condition to initiate microfissuring because there exists the possibilty for the liquid film to eliminate it's supersaturation prior to crack initiation by LFM. Hence it is emphasized that the existence of thin continuous or semicontinuous liquid films on HAZ grain boundaries is an essential but not sufficient condition for the occurrence of HAZ microfissuring. As has been noted many times in the literature, HAZ microfissuring is a complex phenomena entwining more factors than the ones apparently known and further research on different microstructural conditions and different types of alloys is necessary to identify all the factors responsible for this behavior.

The state of the art knowledge, augmented by the findings of the present investigation suggests that the salient microstructural features in causing microfissuring are:

1. Presence of a phase(s) capable of liquation on the grain boundary for example, MC carbides, MNP phosphides etc., 
2. The influence of tramp elements like sulfur and phosphorus leading to (a) decreased solid/liquid, interfacial energy and thus increased wettability (b) increased temperature range of solidification and (c) embrittlement of grain boundaries, 3. Grain size and mean grain boundary curvature - a coarse grain size with no significant grain boundary curvature increasing susceptibility to microfissuring, Additionally, heat treatment has also been shown to influence microfissuring. This is because heat treatment influences all the above mentioned factors. The following sequence of events is proposed for microfissuring of Incoloy 903:

1. Due to the rapid thermal pulse, partial liquation of primary Nb-rich carbides and complete liquation of fine $\mathrm{Nb}$-rich carbides and phosphides occurs on HAZ grain boundaries.

2. The liquid film in the absence of occurrence of LFM, resolidifies in a normal manner by the formation of dendritic gamma and interdendritic MC carbides.

3. Wetting of the grain boundary is enhanced by the presence of boron in addition to the phosphorus as well as sulfur.

4. Phosphorus, sulfur and boron decrease the effective solidus and liquidus temperatures leading to a wider temperature range of solidification. Furthermore, the adsorption of phosphorus and sulfur to solid/liquid interfaces decreases the solid/liquid cohesion and leads to grain boundary embrittlement.

5. An embrittled grain boundary combined with an increased temperature range of solidification, leads to a more crack susceptible microstructure particularly in the very thin film stages that occur during the final stages of solidification of the interdendritic liquid.

6. The combination of a susceptible microstruture as stated above, a coarse grain size, thermal, shrinkage and mechanical stresses generated during cooling leads to microfissuring on the elongated warm worked grain boundaries in the alloy. 


\section{Chapter 5}

\section{CONCLUSIONS}

\section{1: BASE METAL MICROSTRUCTURAL CHARACTERIZATION}

The microstructure of forged Incoloy 903 has been characterized by AEM after various solution treatments.

1. It has been observed that following the standard solution treatment time of one hour after the final thermo-mechanical pass, the necklace structure is preserved up to a solution treatment temperature of $925^{\circ} \mathrm{C}$. The ratio of the volume fraction of the recrystallized grains to warm worked grains was however observed to increase with the increase in solution treatment temperature.

2. It was also observed that in the forged and solution treated ring, the proportion of the fine recrystallized grains to the warm worked grains varied considerably due to the nature of the thermomechanical processing performed to obtain the rolled ring.

3. The grain size of the samples after all the heat treatments has been ascertained. The fine recrystallized grains outlining the warm worked grains in the forged and solution treated ring were observed to have significant grain boundary curvature. The warm worked grain boundaries and the recrystallized grain boundaries in samples solution treated at $1100^{\circ} \mathrm{C}$ were long and straight with minimal grain boundary curvature. However, the recrystallized grains in the samples solution treated for one hour at $975^{\circ} \mathrm{C}$ had significant local grain boundary curvatures owing to the pinning by the fine carbides on the boundaries.

4. Niobium and titanium based coarse MX-type carbides that formed during solidification were found to be aligned in the rolling direction. They have an inner nucleus rich in titanium 
and an outer portion rich in niobium.

5. The chemical composition of the inner nucleus and outer phase have been determined by SEM/EDS quantitative microanalyses. The inner nucleus is observed to be based on $\mathrm{TiN} / \mathrm{Ti}(\mathrm{C}, \mathrm{N})$ while the outer crust of the insoluble is based on $\mathrm{NbC}$.

6. During thermo-mechanical processing, fine carbides and phosphides are observed to precipitate along grain boundaries and prior grain boundaries. The phosphides are observed to be irregular in shape with a great deal of internal defects in them. The carbides were observed to be cuboidal and defect free.

7. The phosphides are observed to be of equi-atomic MNP-type with predominantly $\mathrm{Nb}$ occupying the $\mathrm{M}$ sites and $\mathrm{Co}, \mathrm{Fe}, \mathrm{Ni}$ occupying the $\mathrm{N}$ sites. They have an orthorhombic crystal structure and belong to the pnma space group. Their lattice parameter was determined to be $a=5.99 \AA, b=3.35 \AA$ and $c=7.06 \AA$. The chemical composition of the phosphides has been determined. Based on the chemical composition and the lattice parameter, it is concluded that the phosphides are based on NbCoP with $\mathrm{Fe}$ and Ni substituting partly for Co. Some boron substitution for phosphorus is also likely.

8. If the final solution anneal is performed at $975^{\circ} \mathrm{C} / 1 \mathrm{~h}$, extensive carbide precipitation and phosphide dissolution is observed. The kinetics of carbide precipitation is vastly enhanced by increasing the solution treatment temperature from $840^{\circ} \mathrm{C}$ to $975^{\circ} \mathrm{C}$.

9. The carbides have been observed to be of a MC-type with an average lattice parameter of $4.43 \AA$. The average chemical composition of the fine carbides as determined by TEM/EDS indicated them to be based on $\mathrm{NbC}$ with some substitution of Ti for $\mathrm{M}$ sites. It is also observed that the chemical composition of fine carbides is similar to that of the outer crust of the large insolubles, both being primarily based on $\mathrm{NbC}$.

10. MNP-type phosphides have been observed to re-precipitate extensively on grain boundaries in the as-forged alloy solution treated at $975^{\circ} \mathrm{C} / 1 \mathrm{~h}$ and aged for three hours at $850^{\circ} \mathrm{C}$. These phosphides are observed to be defect free.

11. Long exposures at high temperatures $\left(800-900^{\circ} \mathrm{C}\right)$ result in the formation of needle 
shaped $\delta$ phase. From AEM microanalyses, it is observed that the $\delta$ phase is of the $A_{3} B$ type with $\mathrm{Ni}$ occupying majority of the $\mathrm{A}$ sites and $\mathrm{Nb}$ and $\mathrm{Ti}$ occupying the $\mathrm{B}$ sites.

12. Long exposures at high temperatures $\left(800-900^{\circ} \mathrm{C}\right)$ also result in the formation of an unidentified blocky-intermetallic phase on grain boundaries whose composition has also been determined by AEM microanalyses.

\section{2: CHARACTERIZATION OF FUSION ZONE MICROSTRUCTURE}

1. The microsegregation pattern observed during solidification in the fusion zone indicates that while $\mathrm{Fe}$ and $\mathrm{Co}$ segregate in the gamma dendrites, $\mathrm{Nb}, \mathrm{Ti}$ and $\mathrm{C}$ are extensively rejected into the interdendritic liquid. It has been reasoned that $P$ and $S i$ also segregate extensively into the interdendritic liquid.

2. The elemental partition coefficients at the beginning of solidification has been determined for $\mathrm{Fe}, \mathrm{Ni}, \mathrm{Co}, \mathrm{Nb}, \mathrm{Ti}$ and $\mathrm{Al}$.

3. The major secondary solidification constituent formed in the fusion zone from the interdendritic liquid is cubic, niobium-rich MC carbides. Laves phase was observed only in trace amounts.

4. The interdendritic carbides are observed to form in a characteristic arrow head and lobed morphology. The growth direction of the carbides was determined to be parallel to the cube axes. The arrow head morphology is obtained because of the termination of growth by 111 facets. The Laves phase is generally observed to form at the carbide edges. 5. The composition and lattice parameter of the carbides and Laves phase has been determined by AEM.

6. The chemical analysis and the lattice parameter of the carbides observed in the fusion zone is similar to that of the fine carbides observed in the base metal microstructure.

\section{3: CHARACTERIZATION OF HAZ MICROSTRUCTURES}

1. The grain boundaries in the HAZ showed evidence of liquation during the weld heating cycle and the existence of liquid films enriched in niobium on the grain boundaries. The 
liquid seems to originate primarily by the constitutional liquation of (i) primary carbides (partial), fine MC carbides and MNP phosphides present on the grain boundaries in the forged and solution treated ring (ii) primary carbides (partial) and fine MC carbides in the as-forged alloy solution treated at $975^{\circ} \mathrm{C}$ for one hour and (iii) Primary carbides (partial) and coarsened carbides in samples solution treated in the range of $1 / 2$ to 8 hours at $1100^{\circ} \mathrm{C}$

2. Extensive HAZ microfissuring was observed on the warm worked boundaries of the forged and solution treated ring as well as on the recrystallized boundaries of the forged and solution treated ring further solution treated at $1100^{\circ} \mathrm{C}$ for varying times. The $\mathrm{HAZ}$ microfissures were observed to have a characteristic pleated morphology.

3. Extensive grain boundary liquid film migration and minimal microfissuring was observed in the HAZ on the fine recrystallized grains outlining the warm worked grains in the forged and solution treated ring as well as on the recrystallized grains of the sample solution treated at $975^{\circ} \mathrm{C}$ for one hour.

4. The migrating liquid films leave behind a niobium enriched alloyed zone which appears as thickened regions in $\mathrm{HAZ}$.

5. It is observed that all the microfissures had undergone liquation and resolidification. Images of $\mathrm{Nb} x$-ray maps obtained by SEM suggest the microfissures to be enriched in Nb.

6. Extensive formation of carbide sheets with arrow head and lobed morphologies and as fine precipitates as well as thin solidified films was observed in the HAZ microfissures. The carbides in the arrow head and lobed morphology form as a consequence of dendritic growth, the thin solidified films occur as a consequence of the last stages of solidification accompanied with microfissuring, while the fine carbides precipitate in the solid state. Laves phase was also observed along side carbide sheets in trace amounts.

7. A similar solidification pattern, as observed in the fusion zone, was observed to occur on the liquated grain boundaries that have subsequently developed microfissures. 
8. It was also observed that the $H A Z$ grain boundaries which underwent normal solidification and resisted microfissuring were devoid of the continuous sheets of carbides and/or extensive fine carbide precipitation. Carbides and trace amounts of Laves phase were observed only at discrete pockets on these boundaries. Hence, it is concluded that the prescence of thin continuous and/or semicontinuous liquid films is a necessary condition for HAZ microfissuring.

9. It is thus concluded that the metastable liquid formed on the grain boundaries can relieve its supersaturation in two ways; either by the migration of the liquid film into the matrix leaving behind an alloyed zone or by normal solidification with the formation of dendritic and interdendritic constituents. A primary conclusion of the present investigation is that the solidification mode of the metastable liquid films formed on the HAZ grain boundaries has a major influence on HAZ microfissuring. It can also be concluded that the prescence of continuous and/or semicontinuous liquid films on the HAZ grain boundaries is only a necessary but not sufficient condition for microfissuring to occur.

10. Extensive LFM is observed on the fine recrystallized grain boundaries because of the presence of significant grain boundary curvature. The concentration gradient across the two solid/liquid interfaces and thus the driving force for the initiation of LFM and the velocity of LFM are greatly enhanced in the presence of significant grain boundary curvature. Significant local grain boundary curvatures arise in samples solution treated for one hour at $975^{\circ} \mathrm{C}$ due to the rapid precipitation of fine carbides as well as due to the presence of fine carbides precipitated during thermo-mechanical treatment. These carbides pin the grain boundaries providing significant local grain boundary curvature.

11. The absence of significant grain boundary curvature, the preferred orientation resulting from hot working, the likelihood of increased phosphorus concentration and the preferential adsorption of phosphorus and sulfur to solid/liquid interfaces significantly decrease the coherency stresses that are required for the initiation of LFM on the long warm worked grain boundaries. The grain boundaries in the samples solution treated at $1100^{\circ} \mathrm{C}$ are 
also the equilibrated ones lacking significant curvatures. Thus, LFM is rarely observed on these boundaries. As a consequence, the metastable liquid film equilibrates by normal solidification with the formation of dendritic and interdendritic constituents.

12. The temperature range of solidification of the liquid is significantly decreased with the occurrence of LFM as compared with the normal solidification sequence.

\section{4: MECHANISM(S) FOR HAZ MICROFISSURING}

The salient microstructural features that can cause and/or influence the extent of microfissuring are:

1. The presence of a phase(s) capable of liquation on the grain boundary for example., primary carbide/carbonitride insolubles, fine MC carbides, MNP Phosphides. Alternately, segregation of elements to grain boundaries that signifficantly lower the melting point locally facilitating grain boundary melting.

2. The influence of tramp elements leading to (a) decreased solid/liquid interfacial energy and thus increased wettability (b) increased temperature range of solidification and (c) embrittlement of grain boundaries.

3. Grain size and mean grain boundary curvature; a coarse grain size with no significant grain boundary curvature increases susceptibility to microfissuring while fine grains with significant grain boundary curvature decreases the susceptibility to microfissuring.

4. Additionally, heat treatment has also be shown to influence microfissuring,; solution treatments at higher temperature increasing the susceptibility to $\mathrm{HAZ}$ microfissuring due to the presence of equilibrated straight grain boundaries.

The following sequence of events is proposed for the microfissuring of Incoloy 903 weldments.

1. Due to the rapid thermal pulse, partial liquation of primary Nb-rich carbides and complete liquation of fine $\mathrm{Nb}$-rich carbides and phosphides occurs on HAZ grain boundaries. 
2. The liquid film in the absence of occurrence of LFM, resolidifies in a normal manner by the formation of dendritic gamma and interdendritic MC carbides. The total temperature range of solidification of the liquid is significantly decreased with the occurrence of LFM as compared with the normal solidification sequence.

3. Wetting of the grain boundary is enhanced by the presence of boron in addition to the phosphorus as well as sulfur that are present.

4. The presence of phosphorus and possibly boron and sulfur on liquated grain boundaries (i) increases wetting of the grain boundary by decreasing solid/liquid interfacial energy (ii) decreases the effective solidus and liquidus temperatures thereby increasing the temperature range of solidification (iii) decreases the solid/liquid cohesion and leads to grain boundary embrittlement owing to preferential adsorption to solid/liquid interfaces. These factors hence excacerbates the HAZ microfissuring in Incoloy 903.

5. Long, straight grain boundaries provide a less tortuous path for the spreading of the liquid film and for the crack propagation after crack initiation.

6. An embrittled grain boundary combined with an increased total temperature range of solidification, leads to a more crack susceptible microstructure particularly in the very thin film stages that occur during the final stages of solidification of the interdendritic liquid.

7. The combination of a susceptible microstruture as stated above, a coarse grain size with no significant grain boundary curvature, thermal stresses generated during cooling and the mechanical constraints imposed by the process lead to microfissuring on the long, straight warm worked grain boundaries and equilibrated recrystallized boundaries in the welded alloy. 


\section{REFERENCES}

1. W.A.Owczarscki, D.S.Duvall and C.P Sullivan, Welding Journal, 45, (1966) 145-s.

2. H.D.Brody in "Advances in Welding Science and Technology" edited by S.A.David, ASM, Metals Park, Ohio, 1989.

3. B.A.Chalmers, Principles of Solidification, John Wiley, New York, 1964.

4. M.C.Flemmings,Solidification Processing, McGraw Hill, New York, 1974.

5. K.Easterling, Physical Metallurgy of Welding, Butterworth, London, 1983.

6. N.Christensen, British Welding Journal, 12, (1965) 54.

7 J.F.Lancaster, Metallurgy of Welding, George Allen and Unwin, London, 1980.

8. Welding, ISO Standards Handbook 19, International Organization for Standardization, 1983.

9. Metals Handbook, vol 6, Welding, Brazing and Soldering, ninth edition, ASM, Metals Park, Ohio, 1983.

10. W.F.Savage and C.D.Lundin, Welding Journal, 44, (1965) 433-s.

11. D.A.Krammer, E.C.Masubuchi and R.E.Munroe, "Cracking in High Strength Steel Weldments-A Critical Review" DMIC Report 197, Feb 7th, 1964.

12. W.F.Savage and C.D.Lundin, Welding Journal, 45, (1966) 497-s.

13. E.F.Nippes and W.F.Savage, Welding Journal, 28, (1949) 534-s.

14. E.F.Nippes et. al., Welding Journal, 34, (1954) 183-s.

15. C.H.Kriescher, Welding Journal, 42, (1963) 49-s.

16. D.S.Duvall and W.A.Owczarscki, Welding Journal, 46, (1967) 423-s

17. H.Muesch, Welding of Materials TP 347 mod., Mannesmann Research Institute, Duisburg, 1984.

18. J.R.Donati and G.Zacharie, Revie de Matallurgie, 71, (1974) 905.

19. C.S.Williams, Welding Journal, 42, (1963) 1-s.

20. W.S.Pellini, Foundry, 125, (1952) 124,192,194,196,199. 
21. J.C.Lippold, W.A.Baeslack III and I.Varol, Welding Journal, 71, (1992) 1-s.

22. W.I.Pumphrey and P.H.Jennings, J.Inst.Metals, 75, (1948) 235.

23. J.C.Borland, British Welding Journal, 7, (1960) 508.

24. J.C.Borland, Welding and Metal Fabrication, 47 (1979) 19.

25. J.C.Borland, Welding and Metal Fabrication, 47 (1979) 99.

26. J.Vero, (translation) Metal Ind. (Lond), 48, (1936) 431,491.

27. B.I.Medovar, Automatic Welding, 7, (1954) 12.

28. V.G.Lyuttsau, Liteinoe Proizvodstvo (Russian Casting Production), 2, (1954) 16.

29. V.N.Saveiko, Liteinoe Proizvodstvo (Russian Casting Production), 8, (1960) 453.

30. A.Portevin and M.Dannemuller, J.Inst.Metals, 75, (1948) 949.

31. C.S.Smith, Trans. Amer. Inst. Mining Met. Eng., 175, (1948) 15.

32. J.H.Rogerson and J.C.Borland, TMS-AIME.,227, (1963) 2.

33. J.C.Borland, B.W.R.A. Bulletin, January 1964, 7.

34. H.Tamura and T.Watanabe, Trans. Japan Welding soc., 4, (1973) 30.

35. H.Tamura and T.Watanabe, Trans. Japan Welding soc., 5, (1974) 47.

36. R.D.Thomas Jr., Welding Journal, 63, (1984) 355-s.

37. J.F.King and W.R.Reeds Jr., ORNL Report No TM 6276, 1976.

38. J.C.Lippold, Welding Journal, 62, (1983) 1-s.

39. B.S.Blum and R.H.Witt, Welding Journal, 42, (1963) 365-s.

40. J.A.Brooks, Welding Journal, 53, (1974) 517-s.

41. E.G.Thompson, Welding Journal, 48, (1969) 70-s.

42. R.G.Thompson and S.Genculu, Welding Journal, 62, (1983) 377-s.

43. W.F.Savage and B.M.Krantz, Welding Journal, 31, (1966) 13-s.

44. P.H.Dudley, Ph.D Thesis, Rensselaer Polytechnique Institute, Troy, New York, 1962.

45. A.N.Aronson, M.S Thesis, Rensselaer Polytechnique Institute, Troy, New York, 1963.

46. J.J.Pepe and W.F.Savage, Welding Journal, 46, (1967) 411-s.

47. D.S.Duvall and W.A.Owczarscki, Welding Journal, 45, (1966) 356-s 
48. A.D.Romig, Jr., J.C.Lippold and M.J.Cieslack, Metall. Trans, 19A, (1988) 35.

49. R.Nakkalil and S.P.Gupta, Z.Metallknd., 80, (1989) 37.

50. S.P.Gupta and R.Nakkalil, Acta Metall. Mater., 38, (1990) 1871.

51. R.G.Thomson, J.R.Dobbs and D.E.Mayo, Welding Journal, 66, (1986) 299-s.

52. J.Gordine, Welding Journal, 50, (1971) 480-s.

53. P.J.Valdez and J.B.Steinmen, Proc. Sym. WRC., New York, 1969, 93.

54. D.S.Duvall and W.A.Owczarscki, Welding Journal, 50, (1971) 401-s

55. W.Yeniscavich in "The Superalloys" edited by C.T.Sims and W.C.Hagel, John Wiley and Sons, New York, 1972, p509.

56. T.F.Berry and W.P.Hughes, Welding Journal, 48, (1969) 505-s.

57. K.C.Wu and R.E.Herfert, Welding Journal, 46, (1967) 32-s.

58. W.A.Baeslack III and D.E. Nelson, Metallography, 19, (1986) 371.

59. T.Kelly, in 'Advances in Welding Science and Technology' edited by S.A.David, ASM International, (1986) p623.

60. W.Yeniscavich and C.W.Fox, Proc. Sym. WRC., (1969) 29.

61. W.F.Savage,E.P.Nippes and G.M.Goodwin, Welding Journal, 56, (1977) 245-s.

62. W.R.Apblett and W.S.Pellini, Welding Journal, 33, (1954) 83-s.

63. R.G.Thompson et. al., Welding Journal, 64, (1985) 91-s.

64. R.Thamburaj, Wallace and J.A.Goldak, Int. Met. Rev., 28 (1983) 1.

65. S.J.Mathews, Welding Journal, 54, (1975) 281-s.

66. H.L.Eiselstein and J.K.Bell, Materials in Engineering Design, Nov 1965.

67. H.W.Carpenter, Metal Progress, 1976, 25.

68. D.R.Muzyka, C.R.Whitney and D.K.Schlosser, Journal of Metals, July 1975, 11.

69. H.C.Angus, A.N.Franklin and J.B.Barber, Engineering, May 1970, 8.

70. D.F.Smith and E.F.Clatworthy, Metal Progress, Mar 1981, 33.

71. D.F.Smith, J.P.Morse and R.W.Guenther, Incoloy alloy 907 - Effect of Heat Treatment, Internal Report, Inco Alloys International, Huntington, W.Va, 1982. 
72. K.A.Heck, Incoloy alloy 909 Phase Study, Internal Report, Inco Alloys International, Huntington, W.VA, 1986.

73. Inco Alloys International Ltd., Incoloy 903, Internal Report, 1982.

74. R.G.Baker, Phil. Royal Trans., A282, (1976) 207

75. Y.Nakao, H.Oshige, S.Koga, H.Nishihara and J.Sugitani, J. Japan Welding Soc., 51, (1982) 989.

76. P.G.Shewmon, Diffusion in Solids, J.Williams Book Co., Jinks, Okla, 1983.

77. W.A.Baeslack III, W.P.Lata and S.L.West, Welding Journal, 67, (1988) 77-s.

78. S.C.Ernst, W.A.Baeslack III and J.C.Lippold, Welding Journal, 68, (1989) 418-s.

79. W.Lin, W.A.Baeslack III and J.C.Lippold, II International Conf. on Trends in Welding Research, ASM, Metals Park, Ohio, 1989.

80. N.L.Richards, Bristol Aerospace Ltd., Winnipeg, Manitoba, Personal Communication, 1990.

81. N.L.Richards, R.Nakkalil and M.C.Chaturvedi, To be published.

82. D.E.Appleman and H.T.Evans Jr., Rep. USGS-GD-73-003, NITS, Springfield, Va, 1973.

83. P.J.Postans and R.H.Jeal, in the proceedings of "Forging and Properties of Aerospace Materials" Leeds (1977), The Metals Society, London, p 300.

84. Pearson, W. B., A Handbook of Lattice Spacings and Structures of Metals and Alloys, Pergamon Press, Oxford, 1964.

85. D.W. Andrews, J. Iron and Steel Inst., 163, (1949), 384.

86. G. Brauer, H.Renner and J.Wernet, Z. Anorg. Chem., 277, (1954), 249.

87. J.T.Norton, and A.L.Mowry, J. Metals, 1, (1949), 133.

88. P.Duwez and F.Odell, J. Electochem. Soc., 97, (1950), 299.

89. R.Vincent, Acta Metall., 33, (1985), 1205.

90. R.F. Decker and Sims, C.T., in "The Superalloys" edited by C.T.Sims and W.C.Hagel, John Wiley and Sons, New York, 1972, p 54

91. S.Rundqvist and P.C.Nawapong, Acta Chem. Scand., 20, (1966), 2250. 
92. N.Chaichit et al., Acta Chem. Scand., A 32, (1978), 309.

93. J.H.Westbrook, R.K.Dicerbo and A.J.Peat, The Nickel-Titanium-Silicon system, General Electric Company, GE- 58 RL 2117, 1958.

94. F.X.Spiegel, D.Bardos, and P.A.Beck, Trans. Met. Soc. AIME., 227, (1963), 575.

95. C.B.Shoemaker and D.P.Shoemaker, Acta Cryst., 18, (1965), 900.

96. S.Rundqvist, Acta Chem. Scand., 14, (1960), 1961.

97. S.Rundqvist and P.Tansuriwongs, Acta Chem. Scand., 21, (1967), 813.

98. "Convergent Beam Diffraction of Alloy Phases" (ed J.F. Mansfield) p 76, Adam Hingler, Bristol, (1984).

99. S.Rundqvist, Acta Chem. Scand., 16, (1962), 1.

100.W.Jeitscho, Acta Cryst., B24, (1968), 930.

101. P.I.Krypyakevich et al., Acta Cryst., 27B, (1971), 257.

102.P.I. Krypyakevich, V.YA.Markiv, and V.YA.Melnyk, Dopovidi Akad. Nauk. Ukr. RSR, Ser. A., (1967), 750.

103. R.T.Holt and W.Wallace, IMR, 21, (1976), 1.

104. M.J.Cieslak, G.A.Knorovsky, T.J.Headley and A.D.Romig Jr., Metall. Trans., 17A, (1986) 2107.

105. M.J.Cieslak, T.J.Headley, and A.D.Romig, Jr., Metall. Trans., 17A, (1986) 2035.

106.G.A.Knornovsky, M.J.Cieslak, T.J.Headley, A.D.Romig Jr., and W.F.Hammetter, Metall. Trans, 20A, (1989) 2149.

107. M.J.Cieslak, T.J.Headley, T.Kollie, and A.D.Romig Jr., Metall. Trans., 19A, (1988) 2319.

108. M.J.Cieslak, T.J.Headley, G.A.Knornovsky, A.D.Romig Jr., and T.Kollie, Metall. Trans., 21A, (1990) 479.

109.T.Tanaka et al., Z.Metallknd., 82, (1992) 836.

110.R.G.Thompson, D.E.Mayo, and B.Radhakrishnan, Metall. Trans., 22A, (1991) 557. 111.J.H.Wernick, in 'Intermetallic Compounds', edited by J.H.Westbrook, John Wiley 
and Sons, Inc., New York, 1967, pp. 197-216.

112.S.T.Wlodek, Trans. Am. Soc. Met., 56, (1963) 287.

113. R.F.Decker, in 'Steel Strengthening Mechanisms', Climax Molybdenum, Greenwich, CT, 1969, p. 161.

114.J.A.Brooks and R.W.Krenzer, Welding Journal, 52, (1974) 274-s.

115. B.Radhakrishnan, and R.G.Thompson, Metall. Trans., 22A, (1991) 887.

116. G.Jolley and J.E.Geraghty, in 'Solidification and Casting of Metals', The Metals Society, London, 1979, p. 411.

117. M.J.Cieslak, Welding Journal, 70, (1991) 49-s.

118.D.N.Yoon, and W.J.Huppmann, Acta Metall., 27, (1979) 97.

119. Y.J.Baik, and D.N.Yoon, Acta Metall., 33, (1985) 1911.

120.Y.D.Song, S.T.Ahn, and D.N.Yoon, Acta Metall., 33, (1985) 1907.

121. Y.J.Baik, and D.N.Yoon, Acta Metall., 34, (1986) 2039.

122.W.H.Rhee, Y.D.Song, and D.N.Yoon, Acta Metall., 35, (1987) 57.

123.W.H.Rhee, and D.N.Yoon, Acta Metall., 35, (1987) 1447.

124. T.Muschik, W.A.Kaysser, and T.Hehenkamp, Acta Metall., 37, (1989) 603.

125. Y.J.Baik, and D.N.Yoon, Acta Metall Mater., 38, (1990) 1525.

126. M.Hillert, Scripta Metall., 17, (1983) 237.

127. M.Kuo, and R.A.Fournelle, Acta Metall Mater., 39, (1991) 2835.

128.P.W.Voorhees, R.J.Shaefer, and C.A.Handwerker, 114th TMS-AIME fall meeting, New York, 1985.

129. B.Radhakrishnan, and R.G.Thompson, Scripta Metall. Mater., 24, (1990) 53.

130.C.A.Handwerker, J.W.Cahn, D.N.Yoon, and J.E.Blendell, in "Diffusion in Solids:

Recent Developments" edited by Dayananda, M.D. and Murch, J.E., TMS-AIME, Warrendale, pa, 1986, P 275.

131.D.N.Yoon, J.W.Cahn, C.A.Handwerker, J.E.Blendell and Y.K.Baik in in "Interface "Migration and Contol of Microstructure" edited by C.S.Pande, A.H.King and J.Walter, 
ASM, Metals Park, Ohio, 1986.

132. F.S.Larche and J.W.Cahn, J.Res.NBS 89 (1984) 467.

133. J.W.Gibbs, Scientific Papers, Longman (1906), new edition, Peter Smith, 1984.

134.J.E. Hilliard in "Phase Transformations" ASM, Metals Park, Ohio, 1970, p 497.

135.J.H.Van de Merwe, J.Appl.Phys., 34, (1963) 117.

136.W.A.Jesser and D.Kulmann-Wilsdorf, Physica Status Solidi, 19, (1965) 95.

137.B.R.Patterson and Y.Liu, Metall. Trans., 23A, (1992) 2481.

138. Y.Bretchet and G.R.Purdy, Scripta Metall. Mater., 22, (1988) 1629.

139. N.L.Adams, Physics and Chemistry of Surfaces, Oxford University Press, London, 1942.

140. Handbook of Chemistry and Physics, 53rd Edition, CRC, Ohio, 1972.

141. E.D.Hondros and M.P.Seah, Intl. Met. Rev., 22, (1977) 262.

142.E.D.Hondros in "Precipitation Processes in Solids" edited by K.C.Russel, and H.I.Aaronson, ASM, Metals Park, Ohio, 1979, p1.

143. E.D.Hondros in "Interfaces" edited by K.C.Giffkins, Butterworths, Sydney, 1969, p77.

144.M.P.Seah and E.D.Hondros, Proc.Roy.Soc.Lond., A335, (1973) 91.

145.D.F.Stein and L.A.Heldt, in "Interface Segregation" edited by W.C.Johnson and J.M.Blakely, p 239, ASM, Metals Park, Ohio, 1979.

146. M.Guttmann, Surface Sci.,53, (1975) 213.

147.C.L.Briant and S.K.Banerji in "Embrittlement of Engineering Alloys", (ed) C.L.Briant and S.K.Banerji, p 21, Academic Press, New York, 1983.

148.C.L.Briant and R.P.Messmer, Phil Mag., B42, (1980) 569.

149. R.P.Messmer and C.L.Briant, Acta Metall., 30, (1982) 457.

150. J.A.Brooks, A.W.Thompson and J.C.Williams, Welding Journal, 62, (1983) 220-s.

151.J.C.Borland and R.N.Younger, British Welding Journal, 7, (1960) 22.

152. J.A.Brooks, Welding Journal, 54, (1975) 189-s. 
153. J.A.Brooks and F.J.Lambert, Jr., Welding Journal, 57, (1978) 139-s.

154.J.A.Brooks and A.W.Thompson, Intl Met Rev., 36, 16 (1991).

155.P.D.Merica and R.G.Walternberg, Trans AIME, 71, (1925) 349.

156. K.M.Olson, C.F.Larkins and P.H.Schmitt, Trans ASM, 53, (1961) 349.

157. M.Hansen and K.Anderko, 'Constitution of binary alloys' 2nd ed, McGraw Hill, New York, 1958.

158. E.D.Hondros, M.P.Seah and C. Lea, Met. Mater., Jan (1976) 26.

159. A.Larare, M.Guttman, P.Dumolin and C.Roques-Carmes, Acta Metall., 30, (1982) 685.

160. J.Y.Park, S.Danyluk and D.E.Busch in Physical Metallurgy and Failure Phenomena, edited by R.J.Christofel, E.F.Nippes and H.D.Solomon, G.E. Co., 1979, p 165.

161. H.Kaneko, T.Nishizawa, T.Tamaki and A.Tanifuzi, J.Japan Inst. Metals, 19, (1965) 166.

162. M.Guttmann and M.McLean in "Interfacial Segregation", edited by W.C.Johnson and J.M.Blakely, ASM, Metals Park, Ohio, 1979, p261.

163. C.L.Briant, Acta Metall., 33, (1985) 1241.

164.J.L.Robinson, and M.H.Scott, Phil. Trans.R. Soc., A295, (1980), 105.

165. E.Kny, W.Stolz, R.Stickler, and H.Goretski, J. Vac. Sc. Tech., 17, (1980), 1208.

166. T.Kelly, Welding Journal, 68, (1989) 44-s.

167.R.G.Thompson, Personal Communication, 1992.

168. R.E.Pease, Welding Journal, 36,(1957) 330-s.

169.C.S.Shira, T.J.Morrison and L.A.Weisenberg, Proc. Sym: Effects of Minor Elements on the Weldability of High Nickel Alloys, p 47, WRC, 1967.

170.R.F.Decker, in 'Steel Strengthening Mechanisms', Climax Molybdenum, Greenwich, CT, 1969, p. 147.

171.B.S.Blum, P.Shaw and A.Wickesser, "Improved Methods for Welding Age Hardenable High Temperature Alloys", Report no ASD-TDR-63-601, Republic Aviation Co., 1963. 
172. R.Thamburaj, J.A.Goldak and W.Wallace, SAMPE Q, 4(10), (1979) 6.

173. T.J.Kelly, Welding journal, 69, (1990) 422-s.

174.S.Wlodek and R.D.Field, "The Structure of Rene 220C", R87AEB472, General Electric, Evandale, Ohio.

175. J.F.Radovich and R.C.Carlson, "Microstructural Characterization of Cast Inocnel 718" TM No 85-512, General Electric, Evandale, Ohio.

176.S.A.David et al., Welding journal, 64, (1985) 22-s.

177. M.G. Nicholas and C.M.Old, J.Mat.Sci., 14, (1979) 1.

178. M.H.Kamdar in "Embrittlement of Engineering Alloys", edited by C.L.Briant and S.K.Banerji, Academic Press, New York, 1983, p361.

179. A.R.C.Westwood and M.H.Kamdar, Phil. Mag., 8, (1963) 787.

180.R.G.Thompson, J.J.Cassimus, D.E.Mayo and J.R.Dobbs, Welding Journal, 64, (1985) 91-s. 


\section{Refereed Publications from the Present Thesis}

1. Precipitation of an E Phase in Incoloy 903, R.Nakkalil, N.L.Richards and M.C.Chaturvedi, Scripta Metall. Mater., 25, (1991), pp. 2029-2034.

2. Fusion Zone Microstructure of Electron Beam Welded Incoloy 903, R.Nakkalil, N.L.Richards and M.C.Chaturvedi, Scripta Metall. Mater., 26, (1992) pp 545-550.

3. Grain Boundary Liquid Film Migration During Welding of Incoloy 903, R.Nakkalil, N.L.Richards and M.C.Chaturvedi, Scripta Metall. Mater., 26, (1992) pp 1599-1604.

4. A Study of Precipitates and Insolubles in a Ni-Fe-base Superalloy by Analytical Electron Microscopy, R.Nakkalil, N.L.Richards and M.C.Chaturvedi, Mat Sci Engg A157, (1992) pp 89-100.

5. R.Nakkalil, N.L.Richards and M.C.Chaturvedi, Presented in the session 'Solidification Behavior of Welds' at "The III International Conference on Trends in Welding Research" held at Gatlinburg, Tennessee, 01-05th June 1992. Accepted for publication in 'Welding Science and Technology', ASM International, Meterials Park, Ohio, 1993.

6. Microstructural Characterization of Incoloy 903 weldments, R. Nakkalil, N.L.Richards and M.C.Chaturvedi, Metall. Trans., in press.

7. The Influence of Solidification Mode on Heat affected Zone Microfissuring in a Nickel-Iron-base Superalloy, R.Nakkalil, N.L.Richards and M.C.Chaturvedi, Submitted to Acta Metall. Mater., Dec, 1992.

8. The Effect of Initial Microstructure on Heat affected Zone Microfissuring in a NickelIron-base Superalloy, R.Nakkalil, N.L.Richards and M.C.Chaturvedi, To be submitted. 


\section{Author's Vitae}

\section{NAME: RAGHUNATH NAKKALIL}

Date of Birth : 01 June, 1961

\section{ACADEMIC QUALIFICATIONS}

Master of Technology in Metallurgical Engineering (1984-1986) from Indian Institute of Technology, Kanpur, India, 1987.

Bachelor of Technology in Metallurgical Engineering (1979-1984) from IIT, Kanpur, India, 1985.

\section{THESES, REPORTS AND RESEARCH PUBLICATIONS}

\section{THESES}

1. M.Tech: Morphology and Kinetics of Cellular Dissolution of a Lamellar Precipitate in an Al-18.9 \% Zn Alloy. Department of Metallurgical Engineering, IIT, Kanpur, 1986.

2. B.Tech: Analytical Representation of Hardenability Data on Steels. Department of Metallurgical Engineering, IIT, Kanpur, 1984.

\section{REPORTS}

1. M.N.Bassim and R.Nakkalil: Dynamic Deformation Properties of Rail Steels; A Report submitted to Canadian National Rail Technical Research Centre through University of Manitoba Transport Institute, 1989.

\section{RESEARCH PUBLICATIONS}

\section{Refereed Journal Publications}

1. Microstructural Characterization of Incoloy 903 weldments, R.Nakkalil, N.L.Richards and M.C.Chaturvedi, Metall. Trans., in press.

2. A Study of Precipitates and Insolubles in a Ni-Fe-base Superalloy by Analytical Electron Microscopy, R.Nakkalil, N.L.Richards and M.C.Chaturvedi, Mat Sci Engg A157, (1992) pp 89-100. 
3. Grain Boundary Liquid Film Migration During Welding of Incoloy 903, R.Nakkalil, N.L.Richards and M.C.Chaturvedi, Scripta Metall. Mater., 26, (1992) pp 1599-1604.

4. Fusion Zone Microstructure of Electron Beam Welded Incoloy 903, R.Nakkalil, N.L.Richards and M.C.Chaturvedi, Scripta Metall. Mater., 26, (1992) pp 545-550.

5. Formation of Adiabatic Shear Bands in Eutectoid Steels in High Strain Rate Compression, R.Nakkalil, Acta Metall. Mater., 39, (1991), pp. 2553-2563.

6. Precipitation of an E Phase in Incoloy 903, R.Nakkalil, N.L.Richards and M.C.Chaturvedi, Scripta Metall. Mater., 25, (1991), pp. 2029-2034.

7. A Study of Compression Properties of Rail Steels at High Temperatures and Strain Rates, R.Nakkalil, J.R.Hornaday (Jr.) and M.N.Bassim, Mat Sci Engg., A141, (1991), pp. 247-260.

8. Kinetics of Discontinuous Coarsening of Cellular Precipitate in a Ni-8 at \% In Alloy, S.P.Gupta and R.Nakkalil, Acta Metall. Mater., 38, (1990), pp. 1871-1882.

9. Kinetics of Cellular Dissolution in a Cu-Cd Alloy, R.Nakkalil and S.P.Gupta, Acta Metall., 37, (1989), pp. 1903-1911.

10. Kinetics of Discontinuous Dissolution in an Fe- $\mathrm{Zn}$ Alloy, R. Nakkalil and S.P.Gupta, Z.Metallkd., 80, (1989), pp. 37-47.

11. Kinetics of Cellular Dissolution in an Al-Zn Alloy, R.Nakkalil and S.P.Gupta, Acta Metall., 35, (1987), pp. 2157-2165.

12. The Influence of solidification Mode on Heat affected Zone Microfissuring in a Nickel-Iron-base Superalloy, R.Nakkalil, N.L.Richards and M.C.Chaturvedi, Submitted to Acta Metall. Mater., 1992.

13. The Influence of Welding Parameters on Heat affected Zone Microfissuring in Incoloy 903, N.L.Richards, R.Nakkalil, and M.C.Chaturvedi, Submitted to Metall. Trans., Feb, 1993.

14. The Effect of Initial Microstructure on Heat affected Zone Microfissuring in a Ni-Fe-base Superalloy, R.Nakkalil, N.L.Richards and M.C.Chaturvedi, To be submitted. 
15. Precipitation Behavior in Incoloy 903, R.Nakkalil, N.L.Richards and M.C.Chaturvedi, To be submitted.

\section{Refereed Conference Publications}

1. A Study of Fusion Zone and Heat Affected Zone Microstructures in Electron Beam Welded Incoloy 903, R.Nakkalil, N.L.Richards and M.C.Chaturvedi, in the Proceedings of "The III Int. Conf. on Trends in Welding Research" ASM International, Materials Park, Ohio, 1993 (in press).

2. High Temperature Alloys, M.C.Chaturvedi, N.L.Richards and R.Nakkalil, in the Proc. of "The XIII Canadian Conf on Applied Mechanics", Winnipeg, Canada, 1991, pp. 102-113.

3. Kinetics of Cellular Dissolution in a Cu-Cd Alloy, S.P.Gupta and R.Nakkalil, in "Phase Transformations 87" edited by G.W.Lorimer, Institute of Metals, London, (1988) pp. 269-271.

\section{Conference Presentations}

1. A Study of Fusion Zone and Heat Affected Zone Microstructures in Electron Beam Welded Incoloy 903, R. Nakkalil, N.L.Richards and M.C.Chaturvedi, Presented at

"The III Int. Conf. on Trends in in Welding Research" Gatlinburg, TN, U.S.A, June 1992.

2. High Temperature Alloys, M.C.Chaturvedi, N.L.Richards and R.Nakkalil, Invited presentation at "The XIII Canadian Conference on Applied Mechanics", The University of Manitoba, Winnipeg, Canada, 1991.

3. Kinetics of Cellular Dissolution in a Cu-Cd Alloy, S.P.Gupta and R.Nakkalil, presented at the "The Int.Conf. on Phase Transformations", Cambridge, U.K, June 1987. 